\title{
Low Temperature Metal-Organic Chemical Vapor Deposition Growth Processes for High Efficiency Solar Cells
}

\section{Final Technical Report \\ 1 September 1985 - 30 November 1989}

The Goverment reserves for itself and others acting on its behalf a royalty free, nonexclusive, irrevocable, world-wide license for govermmental purposes to publish, distribute, translate, duplicate, exhibit, and perform any such data oopyrighted by the contractor.

P. D. Dapkus

University of Southern California Los Angeles, California

NREL technical monitor: J. Benner

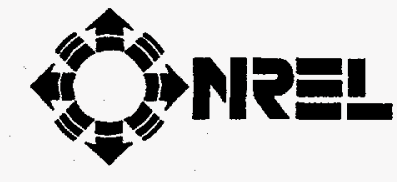

National Renewable Energy Laboratory 1617 Cole Boulevard Golden, Colorado 80401-3393

A Division of Midwest Research Institute Operated for the U.S. Department of Energy under Contract No. DE-AC02-83CH10093

Prepared under Subcontract No. XB-5-05009-3

February 1993

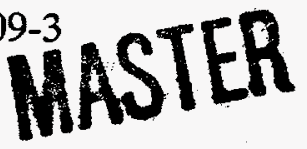

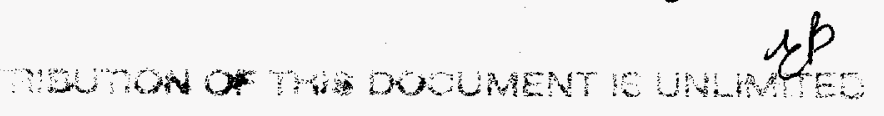


This publication was reproduced from the best available camera-ready copy submitted by the subcontractor and received no editorial review at NREL.

\section{NOTICE}

This report was prepared as an account of work sponsored by an agency of the United States government. Neither the United States government nor any agency thereof, nor any of their employees, makes any warranty, express or implied, or assumes any legal liability or responsibility for the accuracy, completeness, or usefulness of any information, apparatus, product, or process disclosed, or represents that its use would not infringe privately owned rights. Reference herein to any specific commercial product, process, or service by trade name, trademark, manufacturer, or othenwise does not necessarily constitute or imply its endorsement, recommendation, or favoring by the United States government or any agency thereof. The views and opinions of authors expressed herein do not necessarily state or reflect those of the United States government or any agency thereof.

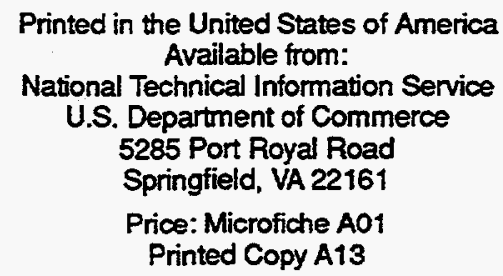

Codes are used for pricing all publications. The code is determined by the number of pages in the publication. Information pertaining to the pricing codes can be found in the current issue of the following publications which are generally available in most libraries: Energy Research Abstracts (ERA); Government Reports Announcements and Index (GRA and 1); Scientific and Technical Abstract Reports (STAR); and publication NTIS-PR-360 available from NTIS at the above address. 


\section{DISCLAIMER}

Portions of this document may be illegible electronic image products. Images are produced from the best available original document. 


\subsection{Introduction}

This report describes the results of a program to develop a more complete understanding of the physical and chemical processes involved in low temperature growth of III-V compounds by MOCVD and to develop a low temperature process that is suitable for the growth of high efficiency solar cells. To accomplish this, the program was structured to develop a better understanding of the chemical reactions involved in MOCVD growth, to develop a model of the processes occurring in the gas phase, to understand the physical kinetics and reactions operative on the surface of the growing crystal and to develop an understanding of the means by which these processes may be altered to reduce the temperature of growth and utilization of toxic hydrides. Lower temperature growth will be required to implement multijunction solar cells concepts and reduced hydride utilization is desirable to reduce the hazard potential of the process and the cost of waste disposal. The basic approach was to develop the required information about the chemical and physical kinetics operative in the gas phase and on the surface by direct physical measurement of the processes whenever possible. Using this basic information, a microscopic model of the MOCVD process and a means to alter the process to achieve the stated goals of lower growth temperature and lower hydride utilization was sought.

The program was set up to include five tasks:

1) MOCVD Growth Process Characterization; 2)Photoenhanced MOCVD Studies; 3) Materials Characterization; 4) Device Fabrication and Characterization; 5) Photovoltaic Training. Most of the goals of the program were met and significant progress was made in defining an approach that would allow both high throughput and high uniformity growth of compound semiconductors at low temperatures. The technical activity was focussed on determining the rates of thermal decomposition of trimethylgallium, on exploring alternate arsenic sources for use in MOCVD, and on empirical studies of atomic layer epitaxy as an approach

\subsection{Decomposition Rates of Trimethylgallium}

In this portion of the project, the thermal decomposition rate of trimethylgallium was measured for the first time using an insitu technique. A molecular beam mass spectrometer was constructed that utilized multistage pumping scheme to sample, in a collisionless way, TMGa/carrier gas mixtures in the hot zone of a furnace. The concentration of TMGa in the gas stream was measured as a function of the temperature in the hot zone to extract the decomposition rate. A unique feature of the results of these experiments is that we are able to determine the activation energies for the fission of each of the methyl radicals from TMGa. In contrast to the original work on this molecule, we find that the activation energies of the three radicals are quite similar. This result will have significant impact on mechanistic studies and the kinetic description of the MOCVD process.

\subsection{Alternate Arsine Sources}

One of the major difficulties of the MOCVD process for high volume applications is the low efficiency of utilization of $V$ hydrides, such as arsine and phosphine. Early in this program, we were able to obtain several different sources of arsine alkyls to study the decomposition rates. These sources were expected to have lower decomposition 
temperatures than arsine and thus would potentially be suitable for low temperature growth of arsenic compounds. The sources we explored were triethylarsine and diethylarsine. A thorough study of the decomposition kinetics was undertaken to determine whether the decomposition path was favorable for the growth of high quality GaAs. Although it was found that diethylarsine decomposed to release an As- $H$ species as the final product and that the decomposition temperature was considerably lower than that of $\mathrm{AsH}_{3}$, these studies were discontinued when manufacturing difficulties made the safe and economical production of diethylarsine impractical.

\subsection{Empirical Studies of Atomic Layer Epitaxy}

During the course of the program, the growth of GaAs by atomic layer epitaxy (ALE) on a companion program funded by the Office of Naval Research was demonstrated. Work by Aoyagi and coworkers of the RIKEN Institute in Japan had shown that growth of GaAs at temperatures as low as $350^{\circ} \mathrm{C}$ could be achieved in ALE with photoexcitation. ALE is characterized by saturated growth, uniform growth that is independent of reactor hydrodynamics, to first order. The process differs from conventional MOCVD in that the wafer is alternately exposed to the columnlll and column $V$ reactants under conditions under which exactly one monolayer is grown per exposure cycle. This saturated growth is achieved by exposing the surface to column III reactants under conditions in which the formation of a Ga rich layer impedes further TMGa surface reactions and results in the formation of one monolayer of $\mathrm{Ga}$. The exposure to arsine then converts the surface to an As rich surface and completes the monolayer.

The potential advantages of this approach to solar cell crystal growth are:

1) Perfect uniformity of deposition ; 2) Separate control and and optimization of the TMGa and $\mathrm{AsH}_{3}$ reactions; 3) Low temperature growth; and 4) High throughput. The last benefit derives from the potential independence of the growth rate upon hydrodynamics that can result from surface saturated reactions. This allows one to scale the process to multiwafer reactors. However high throughput is dependent upon achieving a short monolayer cycle time. in this work we were able to reduce the cycle time to a short as four seconds in an atmospheric pressure reactor. The surface reaction rates at the temperatures at which monolayer saturation could be achieved prevented shorter growth cycle times. Further development of the process will be required to reduce the cycle time to a more practical time $(\sim 1 \mathrm{sec})$. In spite of this needed development, we were able to demonstrate device quality materials and were able to investigate the growth parameters that lead to high quality materials by systematically exploring the properties of thin layers grown under a variety of growth conditions. These studies were carried out under joint sponsorship of SERI and ONR.

\subsection{Organization of the Report}

This report is divided into four sections that describe the studies outlined above. Chapter 2 is a preprint of a paper to be published in the Journal of the Electrochemical Society describing our studies of TMGa decomposition kinetics. Chapter 3 is a reprint describing our studies of the decomposition kinetics of triethylarsine and diethylarsine. Chapter 4 is the PhD dissertation of Steven P. DenBaars describing empirical studies of ALE. This work is jointly sponsored by SERI and ONR. Chapter 5 outlines future work and provides a bibliography of all work supported by this subcontract. 
2.0 On the Thermal Decomposition of Trimethylgallium -- A Molecular Beam Mass Spectroscopy Study

A preprint authored by Qisheng Chen and P. D. Dapkus

Accepted for publication in The Journal of the Electrochemical Society. 
On the Thermal Decomposition of Trimethylgallium

- A Molecular Beam Sampling Mass Spectroscopy Study ${ }^{\dagger}$

\author{
Q.Chen and P.D.Dapkus \\ Department of Material Science and \\ Department of Electrical Engineering \\ University of Southern California
}

Thermal decomposition of trimethylgallium in $\mathrm{H}_{2}, \mathrm{~N}_{2}$ and their admixtures is studied using molecular beam sampling mass spectrometry. Methane and ethane are found as the primary volatile by-products of decomposition The relative amount of $\mathrm{CH}_{4}$ increases and $\mathrm{C}_{2} \mathrm{H}_{6}$ decreases with an increase in $\mathrm{H}_{2}$ concentration relative to $\mathrm{N}_{2}$. The activation energy for the rupture of the first Ga-methyl bond is found to be $E_{1} a=64.6$ $\mathrm{kcal} / \mathrm{mol}$. The activation energies for the rupture of the second and third Ga-methyl bonds are estimated to be $\mathrm{E}_{1 \mathrm{~b}}=52.6 \mathrm{kcal} / \mathrm{mol}$ and $\mathrm{E}_{1 \mathrm{c}}=54.1$ $\mathrm{kcal} / \mathrm{mol}$. Our data reinforce a radical mechanism where TMGa decomposes by way of successively releasing its three methyl radicals.

$\dagger$ This work partially supported by the Office of Naval Research grant N00014-89-J-1229 and the Solar Energy Research Institute subcontract No.XB-5-05009-3. 


\subsection{Introduction}

Despite success in growing device quality III-V semiconductor materials by metalorganic chemical vapor deposition (MOCVD), the understanding of the process is still speculative in nature. Recent interest in atomic layer epitaxy (ALE) of GaAs using trimethylgallium (TMGa) and arsine $\left(\mathrm{AsH}_{3}\right)$ increases the need for accurate information on the chemical properties of $\mathrm{TMGa}$ concerning its stability in the gas phase and its interaction with the substrate surface. Early work of Jacko and Price[1] on TMGa thermal decomposition in toluene showed that only two thirds of methyl radicals were released from TMGa up to $650^{\circ} \mathrm{C}$. The rate constants as a function of temperature for the rupture of the first and second $\mathrm{Ga}$ methyl bonds were determined from the evolution of volatile methylcontaining hydrocarbon products on the assumption that at high temperatures ( $>556{ }^{\circ} \mathrm{C}$ ) the input TMGa quickly converted to DMGa* ( $\mathrm{Ga}\left(\mathrm{CH}_{3}\right)_{2}$ ) in a small fraction of the total contact time. They derived, along with the activation energy for the rupture of the second Ga-methyl bond, an extremely low pre-exponential factor $\left(8.7 \times 10^{7} \mathrm{sec}^{-1}\right)$ for a first order unimolecular reaction that is inconsistent with theoretical expectations[1] and awaits a closer examination. TMGa thermal decomposition was further studied by infrared spectroscopy(IR)[2]--[6], mass spectroscopy[7]--[11], and laser induced fluorescence (LIF)[12]. From the decrease in the mole fraction of TMGa as a function of temperature, DenBaars et al. were able to determine the activation energy for the rupture of the first Ga-methyl bond to be $58-62 \mathrm{kcal} / \mathrm{mol}$. On the contrary, the FTIR data of Mazzarese et al. [6] gave an activation energy for this process as low as $10 \mathrm{kcal} / \mathrm{mol}$. This low value is most likely the manifestation of heterogeneous reaction of $\mathrm{TMGa}$ molecules on the 
stainless steel wall of the apparatus. Other investigations have been qualitative in general. TMGa decomposition is generally found to occur appreciably above about $420^{\circ} \mathrm{C}$. The major volatile low hydrocarbons are $\mathrm{CH}_{4}$ and $\mathrm{C}_{2} \mathrm{H}_{6}$. The decomposition is more complete than previously found in the Ref.[1]. By isotope labeling, Larsen et al[11] was able to identify the main source of $\mathrm{CH}_{4}$ was from the abstraction of atomic $\mathrm{H}(\mathrm{D})$ from $\mathrm{H}_{2}\left(\mathrm{D}_{2}\right)$ by the methyl radicals liberated during TMGa decomposition. Various mechanisms are proposed for the initial step(s), the detailed path(s), and the role of carrier gases in the decomposition. However, a consistent picture along with reliable kinetics information of TMGa gas phase thermal decomposition is not available.

Among the techniques used in the studies of TMGa gas phase decomposition, mass spectrometry has the advantages of being direct in species identification, highly sensitive, and reasonably accurate in quantitative measurement. There are doubts, however, whether the small volume of sample introduced into the ionization chamber through a conventional sampling assembly, such as a long capillary tube, is still representative of the system under investigation. In this paper, we describe a study of TMGa thermal decomposition by utilizing a molecular beam sampling mass spectrometer in conjunction with a plug flow reactor. A differentially-pumped, collision free nozzle assembly, frequently used in chemical kinetics studies to monitor reactive intermediates[13], has been employed in our work. It is fast in response to the change in the gas volume under investigation and able to provide high beam intensity with low background contribution to the supporting vacuum system. The thermal decomposition behavior of $\mathrm{TMGa}$ is examined from room temperature up to $830{ }^{\circ} \mathrm{C}$ in $\mathrm{H}_{2}, \mathrm{~N}_{2}$, and their controlled admixtures. The 
Arrhenius parameters for the process of the breakage of the first Gamethyl bond is found directly from the change in the concentration of $\mathrm{TMGa}$ as a function of temperature. The activation energies for the rupture of the second and third Ga-methyl bonds are also estimated by a two parameter least square fit to the data. A mechanism is proposed to explain the data.

\subsection{Experimental Procedure}

Fig.1 shows the schematic diagram of the experiment setup. The quartz reactor is $5.4 \mathrm{~cm} \mathrm{I.D.} \mathrm{and} 100 \mathrm{~cm}$ in length while the length of the temperature uniform zone can be varied from $67 \mathrm{~cm}$ to $33 \mathrm{~cm}$ by using different furnaces. The large diameter geometry and central positioning of the sampling assembly assures considerably less contribution from the

reactions at the surface. Under typical operation conditions, the average contact time is in the range of a few seconds. The reactor chamber is pumped by a mechanical pump to reduce the pressure drop across the first nozzle for stable molecular beam formation. The volume between the two nozzles is turbo pumped at a pressure of about $5 \times 10^{-5}$ Torr under gas load. The nozzles are made of quartz tube corned at one end and with pin hole through the thin wall at the tip. The first nozzle and the collimator(the second nozzle) are critically aligned for concentricity. When the longer furnace is used, the nozzles are immersed in the hot zone. This allows a nearly in situ probing of the gas mixture under investigation. On the other hand, the nozzle assembly can also be located outside the hot zone when the shorter furnace is used.

Palladium purified $\mathrm{H}_{2}$ or scientific grade $\mathrm{N}_{2}$ (MG 5.5) is used as the carrier gas for TMGa. The TMGa bubbler is kept in an ice bath. All gas 
flows are controlled by mass flow controllers. Total flow is fine adjusted by a metering valve at the inlet of the reactor. The pressure of the reactor is measured by a capacitance barometer. The quadrupole mass spectrometer (model 100-C, UTI) is interfaced to an HP Vectra E5 PC with a SPECTRALINK unit (UTI). The data acquisition is thus made simple, quick, accurate, and reproducible.

Before each run, the reactor tube is cleaned with a mixture of HF and $\mathrm{HNO}_{3}(1: 9)$, thoroughly rinsed with $\mathrm{DI}$ water, and blown dry with $\mathrm{N}_{2}$. The tube was then heated to $200{ }^{\circ} \mathrm{C}$ under vacuum for degassing. The molecular beam sampling system is first tested for its behavior of beam intensity with respect to pressure and mole fraction. Trial runs were also done to calibrate the entire system with $\mathrm{H}_{2}, \mathrm{CH}_{4}, \mathrm{C}_{2} \mathrm{H}_{6}, \mathrm{~N}_{2}$, and TMGa in order to obtain a complete knowledge of the temperature dependent mass selectivity of the sampler. Experiments on TMGa thermal decomposition are generally carried out by monitoring multiple peak intensities at constant pressure and fixed $\mathrm{TMGa}$ to $\mathrm{H}_{2}$ ratio with varying temperature or at constant pressure and temperature while varying the TMGa to $\mathrm{H}_{2}$ ratio. Peak intensities are corrected for the temperature dependent mass selectivity and the thermal expansion of the gas. The corrections are made as if the effluent gas is cooled to room temperature.

\subsection{Experimental Results}

The molecular beam sampling system showed a linear dependence of the beam intensity on total pressure over the pressure range of operation ( $0.01-2$ Torr.) for a gas mixture of $\mathrm{H}_{2}$ saturated with TMGa maintained at $0{ }^{\circ} \mathrm{C}$ when the $\mathrm{m} / \mathrm{e}=99$ ion from $\mathrm{TMGa}$ is monitored. A slightly different test is also done for a $\mathrm{H}_{2} / \mathrm{N}_{2}$ mixture where a variable amount of $\mathrm{H}_{2}$ is 
introduced into a $\mathrm{N}_{2}$ flow but the total pressure remains constant. The detected $\mathrm{H}_{2}$ signal ( $\mathrm{m} / \mathrm{e}=2$ ) changes linearly with the change in the $\mathrm{H}_{2}$ mole fraction. This linear relationship between the beam intensity and the volume density of molecules was derived by Kantrovitz and Grey[14] and refined by Parker et al. [15].

Typical mass spectra for TMGa in $\mathrm{N}_{2}$ at room temperature $\left(24^{\circ} \mathrm{C}\right)$ and the temperature where considerable decomposition occurs $\left(\right.$ e.g $\left.720^{\circ} \mathrm{C}\right)$ are shown in Fig.2 (a) and (b). One task in using a mass spectrometer to obtain quantitative information is to identify peaks that best represent the molecular species of the gas phase sampled. Even at low ionization voltage (nominal $29 \mathrm{~V}$ measured from the terminals in the instrument console) where further reduction of the ionization voltage would result in drastic fall off in the sensitivity for this particular gas analyzer, TMGa produces only a very weak peak at the molecular ion position, i.e. $\mathrm{m} / \mathrm{e}=114$ and 116 . Consequently the $\mathrm{Ga}\left(\mathrm{CH}_{3}\right)_{2}+$ ions at $\mathrm{m} / \mathrm{e}=99$ and 101 are monitored as a measure of the remaining TMGa concentration. The low hydrocarbons evolving from the reaction are measured by the changes in the peak intensities at $\mathrm{m} / \mathrm{e}=16$ and 30 . They are identified as mainly $\mathrm{CH}_{4}$ and $\mathrm{C}_{2} \mathrm{H}_{6}$ according to the spectra obtained from $0.409 \% \mathrm{CH}_{4}$ in $\mathrm{H}_{2}$ and $0.5 \% \mathrm{C}_{2} \mathrm{H}_{6}$ in $\mathrm{H}_{2}$ standards, Fig.3 (a) and (b). The molecular ion of $\mathrm{C}_{2} \mathrm{H}_{6}$ is monitored instead of the stronger peak at $\mathrm{m} / \mathrm{e}=28$ because the ionization pattern of TMGa at $\mathrm{m} / \mathrm{e}=28$ would interfere with the measurement and also because $\mathrm{N}_{2}$ will be used as carrier gas for some relevant runs. The multiple peak monitoring and auto ranging features of the computer data acquisition are especially useful in this case to pick up a weak peak adjacent to a strong peak. The signal amplitudes for all of the $\mathrm{Ga}$ containing ion species decreased with the same dependence as the temperature was increased. This 
allows us to conclude that the measured $\mathrm{Ga}$ containing species all originate from the undecomposed parent molecule and that the signals from sampled intermediates are lower by at least two orders of magnitudes. In other words, the detection of any $\mathrm{Ga}$ containing species as the products of the thermal decomposition is masked by the high background contributed by the TMGa ionization pattern at nominal ionization voltage of $29 \mathrm{~V}$, (cf. Fig.2 (a)).

When the peak intensities of remaining $T M G a$ at different temperatures are plotted, Fig. 4 results. It is evident that appreciable decomposition does not occur until about $430{ }^{\circ} \mathrm{C}$ for TMGa in $\mathrm{H}_{2}$ and 480 ${ }^{\circ} \mathrm{C}$ for $\mathrm{TMGa}$ in $\mathrm{N}_{2}$. TMGa molecules disappear almost completely from the gas stream above about $610^{\circ} \mathrm{C}$. The data as shown in Fig. 4 can be analyzed to yield apparent activation energies characteristic of the rate at which molecular TMGa disappears. The results are listed in Table I for several TMGa to $\mathrm{H}_{2}$ mole ratios, where a known amount of $\mathrm{N}_{2}$ is used as part or the whole of the carrier gas.

The involvement of $\mathrm{H}_{2}$ in the reaction under the conditions used in our experiment can best be seen from the result of the experiment where variable admixture of $\mathrm{TMGa} / \mathrm{H}_{2} / \mathrm{N}_{2}$ is fed into the reactor at $\mathrm{T}=600{ }^{\circ} \mathrm{C}$ and $\mathrm{P}=2$ Torr. The intensity of the remaining TMGa dropped drastically when the dose of $\mathrm{H}_{2}$ is increased as shown in Fig.5. The change in the relative amount of $\mathrm{H}_{2}$ in the input gas mixture also changes the relative amount of $\mathrm{CH}_{4}$ and $\mathrm{C}_{2} \mathrm{H}_{6}$ in the products as shown in Fig.6. The advantage of plotting the abscissa in terms of $[\mathrm{TMGa}]_{0} /\left([\mathrm{TMGa}]_{0}+\left[\mathrm{H}_{2}\right]_{0}\right)$ in the Fig. 6 will become apparent during the discussion. 
We can define a quantity which is the sum of the moles of $\mathrm{CH}_{4}$ and two times the moles of $\mathrm{C}_{2} \mathrm{H}_{6}$ produced from the decomposition of each mole of the input $\mathrm{TMGa}$,

$$
\operatorname{PRD}\left(\mathrm{CH}_{3}\right)=\frac{\left[\mathrm{CH}_{4}\right]}{[\mathrm{TMGa}]_{0}}+2 \frac{\left[\mathrm{C}_{2} \mathrm{H}_{6}\right]}{[\mathrm{TMGa}]_{0}}
$$

This quantity is then a measure of the number of moles of methyl radicals released for each mole of TMGa consumed, ignoring much smaller contributions from other low hydrocarbons. This is a true measure of the over all decomposition progress, irrespective of the details of how these products are formed. The quantity $\operatorname{PRD}\left(\mathrm{CH}_{3}\right)$ is plotted in Fig.7 as a function of reactor temperature. In principle, as detailed in the Appendix, the apparent Arrhenius parameters for successive release of the three methyl radicals can be found independently by a multiple parameter least square curve fit. To avoid the inaccuracies that result from the peculiar functional behavior of the Arrhenius expressions for the rate constants, we choose to use the rate constant expression found from the temperature dependence of the remaining reactant (TMGa) as the apparent rate constant for the breakage of the first Ga-methyl bond, $\mathrm{k}_{1 \mathrm{a}}$. We also estimate the Arrhenius pre-exponential factors for the unimolecular reactions of the rupture of the second and third Ga-methyl bonds, $A_{1 b}$ and $A_{1 c}$, to be as $4 \times 10^{14} \mathrm{sec}^{-1}$ and $1 \times 10^{14} \mathrm{sec}^{-1}$ respectively by adopting a statistical thermodynamics approach as given, for example, by Benson[16]. The two parameter least square fits to the data as plotted in Fig.7 give the activation energies for the reactions of the rupture of the second and the third Gamethyl bonds as $\mathrm{E}_{1 \mathrm{~b}}=52.6 \mathrm{kcal} / \mathrm{mol}$ and $\mathrm{E}_{1 \mathrm{c}}=54.1 \mathrm{kcal} / \mathrm{mol} \mathrm{respectively}$. 
The sum of the squared errors between the fitted expression and the experimental data has single sharp minimum with respect to $E_{1 b}$ and $E_{1 c}$ and the values of $E_{1 b}$ and $E_{1 c}$ are not very sensitive to the changes in $A_{1 b}$ and $A_{1 c}$. It is important to note that the sum of the three activation energies thus determined independently is $171.3 \mathrm{kcal} / \mathrm{mol}$, in agreement with the $172.4 \mathrm{kcal} / \mathrm{mol}$ thermochemical measurement of Long[17], the value of $175.7 \mathrm{kcal} / \mathrm{mol}$ summarized by Tuck[18], the value of $183.9 \mathrm{kcal} / \mathrm{mol}$ that is derived according to the compilation of Pilcher and Skinner[19], and the value of $176.1 \mathrm{kcal} / \mathrm{mol}$ that is calculated from the compilation of Tirtowidjojo and Pollard[20]. An electron impact study also gave a value of $166.8 \mathrm{kcal} / \mathrm{mol}$ for this quantity[21].

The completion of the decomposition is also tested by putting a few small pieces of Si wafers and quartz plates in the uniform hot zone of the reactor for several runs. They are taken out for evaluation. Droplets of diameters up to about $6 \mu \mathrm{m}$ can be seen on the surface after decomposition in either $\mathrm{H}_{2}$ or $\mathrm{N}_{2}$. The overall film on the quartz plates is electrically conducting. The deposit dissolves slowly in $\mathrm{HCl}$ with little residue. On the contrary, the deposit on the entrance section of the reactor wall, where the rising shoulder of the temperature profile occurs, leaves more residue even after the etch in 1:9 mixture of $\mathrm{HF}$ and $\mathrm{HNO}_{3}$. This is especially true after decomposition in $\mathrm{N}_{2}$. These observations suggests that the decomposition is complete with the production of $\mathrm{Ga}$ atoms under most conditions used here, that certain reactions involving $\mathrm{CH}_{3}$ are more pronounced in $\mathrm{N}_{2}$ ambient (cf. Reaction(5) below), and that any polymeric deposit tend to form in or diffuse to the low teperature region.

\subsection{Discussion}


To explain our data, we propose the following minimal reaction scheme specifically for TMGa decomposition in $\mathrm{H}_{2}$ with relatively long contact time so that steady state conditions are likely to be established:

$$
\begin{aligned}
& \mathrm{TMGa} \rightarrow \mathrm{DMGa}^{*}+\mathrm{CH}_{3}{ }^{*} \\
& \mathrm{DMGa}^{*} \rightarrow \mathrm{MMGa}^{*}+\mathrm{CH}_{3}{ }^{*}
\end{aligned}
$$

The decomposition in $\mathrm{N}_{2}$ (non-reacting carrier gas) can also be discussed by ignoring the reactions originating from the presence of $\mathrm{H}_{2}$. The reaction (5) is included to account for the methane formed when TMGa decomposes in $\mathrm{N}_{2}$. More reactions may be added to account for small amount of $\mathrm{C}_{2} \mathrm{H}_{4}$ and higher hydrocarbons $(\mathrm{m} / \mathrm{e}=40-44)$ seen in the spectra, but they are ignored here because of their low relative concentrations.

The inclusion of methyl radicals in the intermediates demands a few words of explanation. Butler and co-workers reported the observation of free $\mathrm{CH}_{3}{ }^{*}$ from TMGa thermal decomposition over a hot susceptor using infrared-diode laser spectroscopy[5]. Although the direct observation of free methyl radicals by mass spectroscopy was claimed by the publications of Eltenton[22] and Lossing et al.[23] from thermally decomposed mixture 
of tetramethyllead and ethane and dimethylmercury respectively, recent application of the technique is rare. We attempted to detect the net $\mathrm{CH}_{3}{ }^{*}$ signal over the parent background of $T M G a, \mathrm{CH}_{4}$, and $\mathrm{C}_{2} \mathrm{H}_{6}$ using a very low ionization voltage, nominal $18 \mathrm{~V}$ and the lowest reasonable value for this gas analyzer. After analyzing the ionization efficiency curves of TMGa, $\mathrm{CH}_{4}$, and $\mathrm{C}_{2} \mathrm{H}_{6}$ obtained with this mass spectrometer, we find that the appearance potential of $\mathrm{CH}_{3}{ }^{+}$ion from molecular $\mathrm{TMGa}$ is about 12.7 ev, greater than the ionization potential of free $\mathrm{CH}_{3}{ }^{*}$ (9.83 ev [24]) by only a small margin, $2.9 \mathrm{ev}$, as compared to the corresponding differences ( $\left.\mathrm{AP}(\mathrm{X})-\operatorname{IP}\left(\mathrm{CH}^{*}\right)\right)$ for $\mathrm{X}=\mathrm{CH}_{4}, 4.6 \mathrm{ev}$, and $\mathrm{X}=\mathrm{C}_{2} \mathrm{H}_{6}, 4.4 \mathrm{ev}$. Consequently, there will always be appreciable contribution to the $\mathrm{m} / \mathrm{e}=15$ signal from the ionization cracking pattern of molecular TMGa and, at an appropriate low ionization voltage, only the contribution from TMGa is important relative to the contributions from $\mathrm{CH}_{4}$ and $\mathrm{C}_{2} \mathrm{H}_{6}$ which are present during the thermal decomposition. By operating the mass spectrometer at low ionization voltage we are able to obtain a net $\mathrm{m} / \mathrm{e}=15$ signal after subtracting the contribution from the remaining molecular TMGa. The net $m / e=15$ signal starts to appear at the temperature at which decomposition occurs appreciably. It increases with $\mathrm{T}$ at first and then decreases after passing a maximum, resembling the behavior of $\mathrm{CH}_{3}{ }^{*}$ observed by Butler et al. Methyl radicals also manifest themselves in the formation of such observed low hydrocarbon products as $\mathrm{CH}_{4}$ and $\mathrm{C}_{2} \mathrm{H}_{6}$. These products are not likely to be formed directly from TMGa molecules through bond rearrangement. The additional energy in distorting the simple near planar structure of TMGa is too great to make this a favorable path. The molecular orbital calculation of Tsuda et al. [25] also showed process (1a) as the initial step in TMGa decomposition. 
The temperature at which the decomposition becomes appreciable is related to the absolute sensitivity of the detection techniques and the contact time . In Fig.4, we compare the decomposition in $\mathrm{H}_{2}$ and $\mathrm{N}_{2}$ for the same contact time. TMGa decomposition in $\mathrm{H}_{2}$ commences at lower temperatures than in $N_{2}$. This shift of the curve to lower temperatures in Fig.4 is occasionally disputed as the effect of the difference in thermal and hydrodynamic properties of the carrier gases[8]. The change in relative amount of $\mathrm{CH}_{4}$ versus $\mathrm{C}_{2} \mathrm{H}_{6}$ generated for different $\mathrm{TMGa} / \mathrm{N}_{2} / \mathrm{H}_{2}$ mixtures as shown in Fig. 6 is, however, a strong indication of the involvement of $\mathrm{H}_{2}$ in the reactions. This confirms the result by Larsen et al[11]. Here the data are presented in terms of [TMGa $]_{0} /\left([\mathrm{TMGa}]_{0}+\left[\mathrm{H}_{2}\right]_{0}\right)$ since an analysis by taking only the Eqn.s (1a) through (4) assumes neat form when the $\left[\mathrm{CH}_{4}\right] / 3$ [TMGa $]_{0}$ and $\left[\mathrm{C}_{2} \mathrm{H}_{6}\right] / 1.5$ [TMGa $]_{0}$ are expressed as functions of TMGa $]_{0} /\left([\mathrm{TMGa}]_{0}+\left[\mathrm{H}_{2}\right]_{0}\right)$. Such analysis shows that the Eqns. (1a) through (4) describe the basic behavior of TMGa thermal decomposition in presence of $\mathrm{H}_{2}$. The direct reaction between $\mathrm{H}_{2}$ and $\mathrm{TMGa}$ is ruled out by energy considerations. However, reaction (2) has a low activation energy around $10 \mathrm{kcal} / \mathrm{mol}[26]-[28]$. It will compete with reactions (3) and (5) for the supply of $\mathrm{CH}_{3}{ }^{*}$ and generate atomic $\mathrm{H}$. The kinetics of attack on $\left(\mathrm{CH}_{3}\right)_{\mathrm{X}} \mathrm{Ga}$ by atomic $\mathrm{H}$, reaction (6), are not known. It is considered as an important path in the destruction of atomic $\mathrm{H}$ because the activation energy for radical-molecule reaction is generally low. At low concentration of $\mathrm{H}_{2}$, the recombination of methyl radicals to form $\mathrm{C}_{2} \mathrm{H}_{6}$ dominates. A small amount of $\mathrm{CH}_{4}$ forms from the abstraction of $\mathrm{H}$ by $\mathrm{CH}_{3}{ }^{*}$ from $\mathrm{Ga}$ containing hydrocarbons, most likely $\mathrm{Ga}\left(\mathrm{CH}_{3}\right)^{*}$, i.e. $\mathrm{x}=1$ in the reaction (5) because at this later stage of TMGa decomposition, the chance of that a gallium containing species will collide with methyl radicals is the highest. 
A reasonable carbon balance supports this point. Since we did not observe the accumulation of either $\mathrm{Ga}\left(\mathrm{CH}_{2}\right)^{*}$ or $\mathrm{CH}_{2}{ }^{*}$ species in the gas phase, we have to assume that the $\mathrm{Ga}\left(\mathrm{CH}_{2}\right)^{*}$ is not volatile and the $\mathrm{Ga}-\mathrm{CH}_{2}$ bond is stable. This accounts for the dark deposit on the reactor wall at the entrance of the hot zone, especially after TMGa decomposition in $\mathrm{N}_{2}$. This deposit dissolves partially in mixture of $\mathrm{HF}$ and $\mathrm{HNO}_{3}$ with films of polymer-resembling residue. At high $\mathrm{H}_{2}$ concentration, reactions (2), (3), and (6) will dominate and $\mathrm{CH}_{4}$ is then the primary low hydrocarbon species.

Frequently, a direct test of the effect of carrier gas on properties of the grown epilayers by conventional MOCVD hydride growth shows little dependence on carrier gas[29][30]. This occurs because $\mathrm{AsH}_{3}$ is a more effective source of atomic $\mathrm{H}$ than $\mathrm{H}_{2}$ itself and facilitates the removal of $\mathrm{CH}_{3}$ from the surface. It may also be pointed out that the decomposition behavior discussed here is obtained with moderate contact time. It is expected that with very short contact time the reactions between intermediates and carrier gas will be diminished. The effect of carrier gas on TMGa thermal decomposition becomes less perceptible.

The attack on TMGa by atomic $\mathrm{H}$ could explain the variation of the apparent activation energies with $\mathrm{TMGa}$ to $\mathrm{H}_{2}$ ratios. In the extreme of TMGa thermal decomposition in $\mathrm{N}_{2}$, only reactions (1a), (1b), (1c), (4), and (5) are to be considered. The apparent rate constant is obviously $\mathrm{k}_{1 \mathrm{a}}$ and the measured apparent activation energy can be taken as the activation energy for reaction (1a), i.e. $E_{1 a}=64.6 \mathrm{kcal} / \mathrm{mol}$. The determination of first order rate parameter from temperature dependence of the reactants does not involve any assumption on reaction details in producing products. Neither does it require an absolute calibration of the monitoring system. 
This type of measurement and analysis is easier and probably be more reliable than that from the temperature dependence of the products. The reported values for the $E_{1 \mathrm{a}}$ are $59.5 \mathrm{kcal} / \mathrm{mol}[1]$ and $58-62 \mathrm{kcal} / \mathrm{mol}[4]$. A molecular orbital calculation resulted in $64.9 \mathrm{kcal} / \mathrm{mol}$. The agreement is reasonable.

In Ref.[1], the rate of the reaction (1b), $\mathrm{k}_{1 \mathrm{~b}}$, was assumed to be much slower than that of the reaction (1a) in high temperature range such that all input TMGa is converted to DMGa in a small fraction of the contact time. A low activation energy for the reaction (1b) was deduced from the data analysis using this assumption. The result that $E_{1 b}$ is much lower than $E_{1 a}$ would generally mean that the rate of the reaction (1b) should be much faster than that of the reaction (1a) - contradicting the original assumption - unless the reactions (1a) and (1b) proceed by a totally different mechanism. Not only is this self inconsistency noteworthy, but also the absence of $\mathrm{Ga}\left(\mathrm{CH}_{3}\right)_{2}{ }^{*}$ and $\mathrm{Ga}\left(\mathrm{CH}_{3}\right)^{*}$ species from various diagnostic techniques would suggest that they are in relatively low concentrations. An equilibrium analysis of MOCVD[20] using the available kinetic data, however, predicts $\mathrm{Ga}\left(\mathrm{CH}_{3}\right)^{*}$ at more than quarter of the $\mathrm{CH}_{4}$ concentration. This high level of $\mathrm{Ga}\left(\mathrm{CH}_{3}\right)^{*}$ has never been observed experimentally. Our values of $E_{1 a}, E_{1 b}$, and $E_{1 c}$ are consistent with the picture that TMGa decomposes by releasing successively its three methyl radicals along the whole length of the hot zone and the intermediate species $\mathrm{Ga}\left(\mathrm{CH}_{3}\right)_{2}{ }^{*}$ and $\mathrm{Ga}\left(\mathrm{CH}_{3}\right)^{*}$ do not survive for long the environment that has caused the rupture of the first Ga-methyl bond. The stabilities of $\mathrm{Ga}\left(\mathrm{CH}_{3}\right)_{2}{ }^{*}$ and $\mathrm{Ga}\left(\mathrm{CH}_{3}\right)^{*}$ are different only by a small margin in favor of last Ga-methyl bond. Finally the presence of atomic $\mathrm{Ga}$ in the final 
products also points out the complete breakdown of TMGa above about 610 ${ }^{\circ} \mathrm{C}$.

\subsection{Conclusion}

An intensive study has been carried out on thermal decomposition of TMGa in $\mathrm{H}_{2}, \mathrm{~N}_{2}$, and their admixtures by using molecular beam sampling mass spectrometry. Methane and ethane are found as the primary volatile hydrocarbon products. At high enough temperatures, the relative amounts of $\mathrm{CH}_{4}$ and $\mathrm{C}_{2} \mathrm{H}_{6}$ formed in the gas phase are affected by the presence of $\mathrm{H}_{2}$. The amount of $\mathrm{CH}_{4}$ increases and $\mathrm{C}_{2} \mathrm{H}_{6}$ decreases while the mole fraction of $\mathrm{H}_{2}$ increases in a $\mathrm{TMGa} / \mathrm{H}_{2} / \mathrm{N}_{2}$ mixture. The measured apparent activation energies from the temperature dependence of the remaining reactant differ for decompositions occurring in $\mathrm{H}_{2}$ and $\mathrm{N}_{2}$. This difference is explained qualitatively by the participation of $\mathrm{H}_{2}$ in the reaction specifically by the attack of TMGa by atomic $\mathrm{H}$. The apparent activation energy obtained from the temperature dependence of remaining reactant for TMGa in $\mathrm{N}_{2}$ is taken as the activation energy for the process of rupturing the first $\mathrm{Ga}$-methyl bond, i.e. $E_{1 \mathrm{a}}=64.6 \mathrm{kcal} / \mathrm{mol}$. The activation energies for the reactions of breaking the second and the third Ga-methyl bond are also estimated independently to be $E_{1 b}=52.6 \mathrm{kcal} / \mathrm{mol}$ and $\mathrm{E}_{1 \mathrm{c}}=54.1 \mathrm{kcal} / \mathrm{mol}$ from the temperature dependence of the volatile hydrocarbon products. Our data reinforce a radical mechanism where TMGa decomposes by way of releasing successively its three methyl radicals. The rupture of the first Ga-methyl bond is, by far, the rate limiting step. 
Table I: Apparent First Order Rate Constants

$$
\left(\operatorname{lnk}\left(\sec ^{-1}\right)=\ln A-\frac{E_{a}}{R T}\right) \text {. }
$$

\begin{tabular}{|c|c|c|c|c|}
\hline$\frac{[\mathrm{TMGa}]}{\left[\mathrm{H}_{2}\right]}$ & $0.304 \%$ & $1.713 \%$ & $8.03 \%$ & $\infty$ \\
\hline $\ln \mathrm{A}$ & 31.3 & 31.7 & 31.7 & 37.9 \\
\hline $\mathrm{Ea}\left(\frac{\mathrm{kcal}}{\mathrm{mol}}\right)$ & 50.9 & 51.5 & 54.8 & 64.6 \\
\hline
\end{tabular}



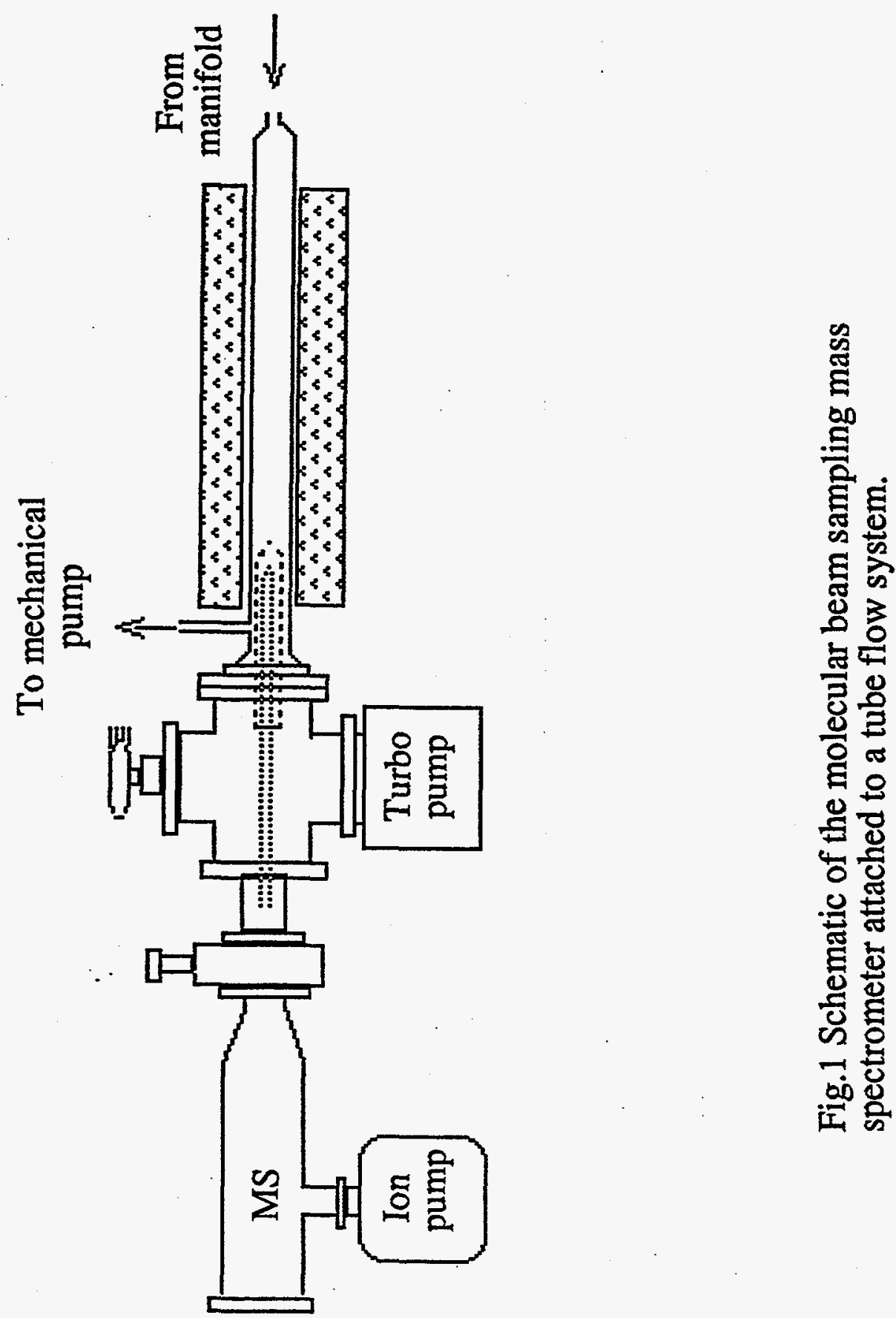


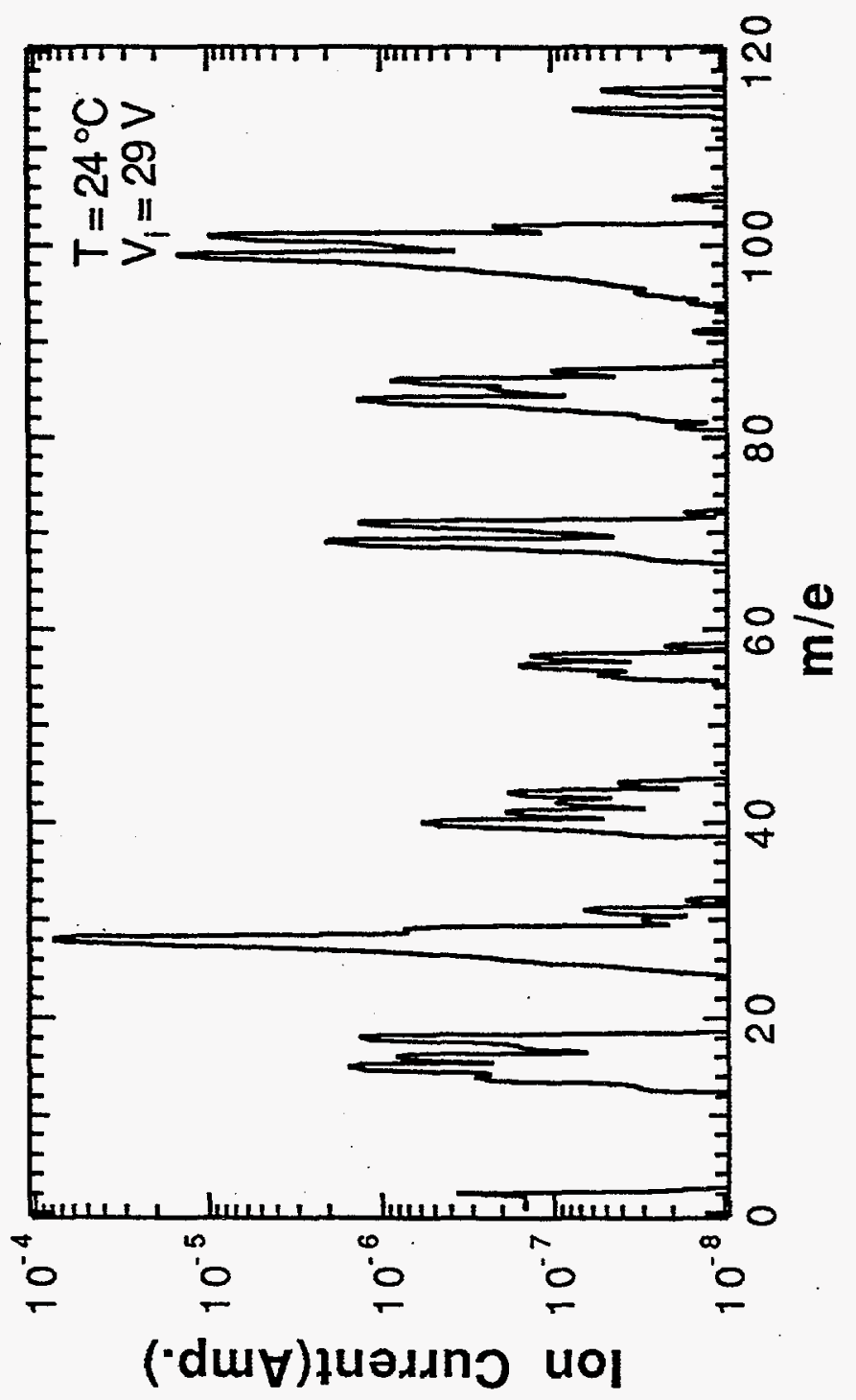

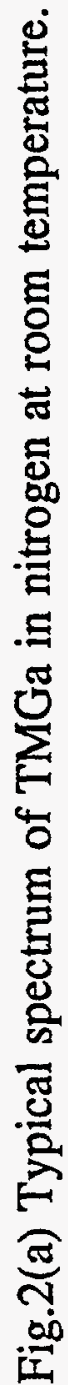




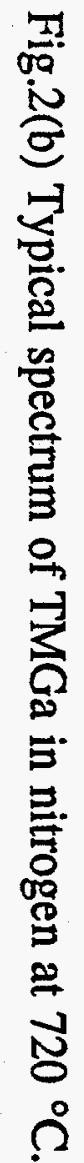

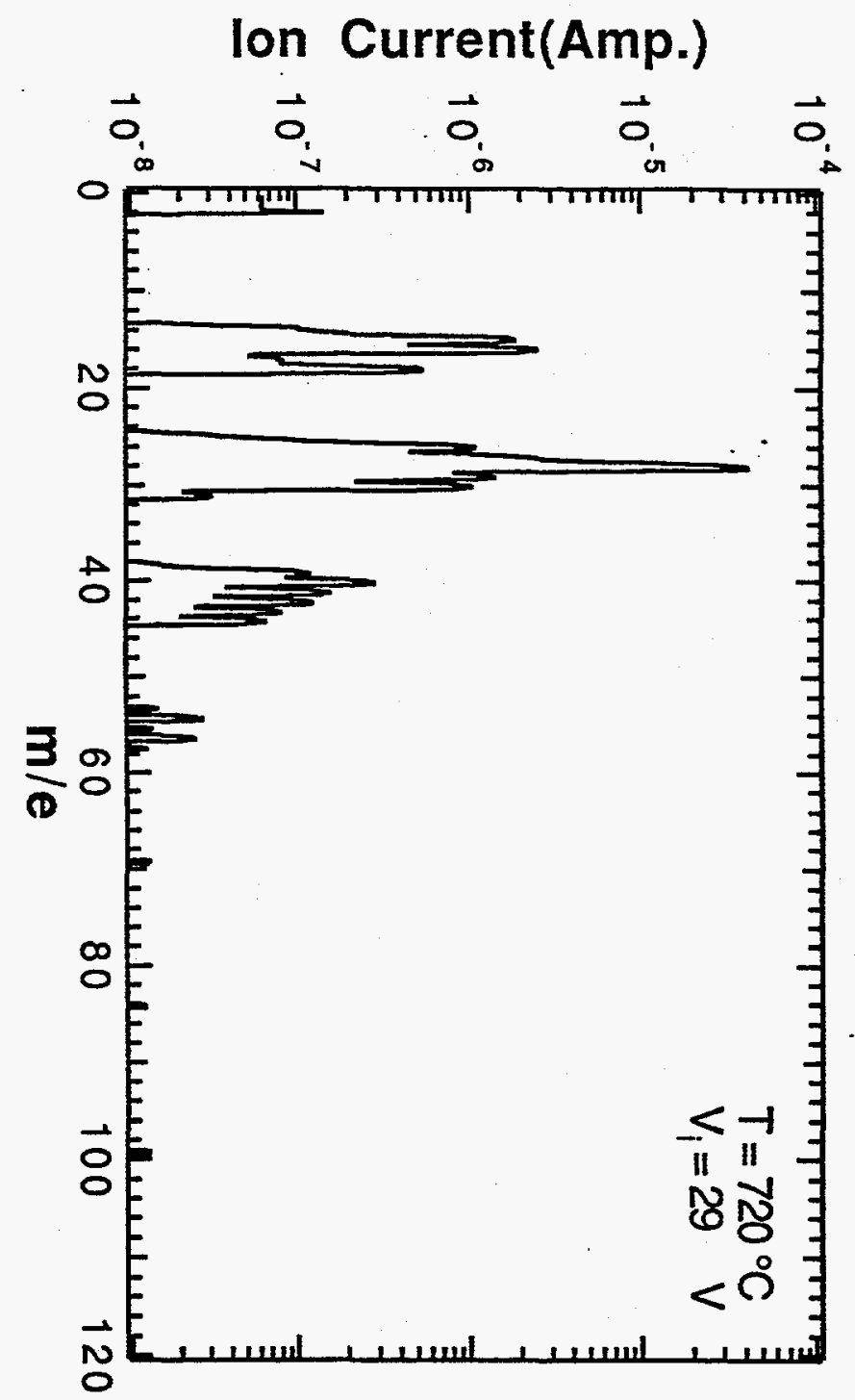




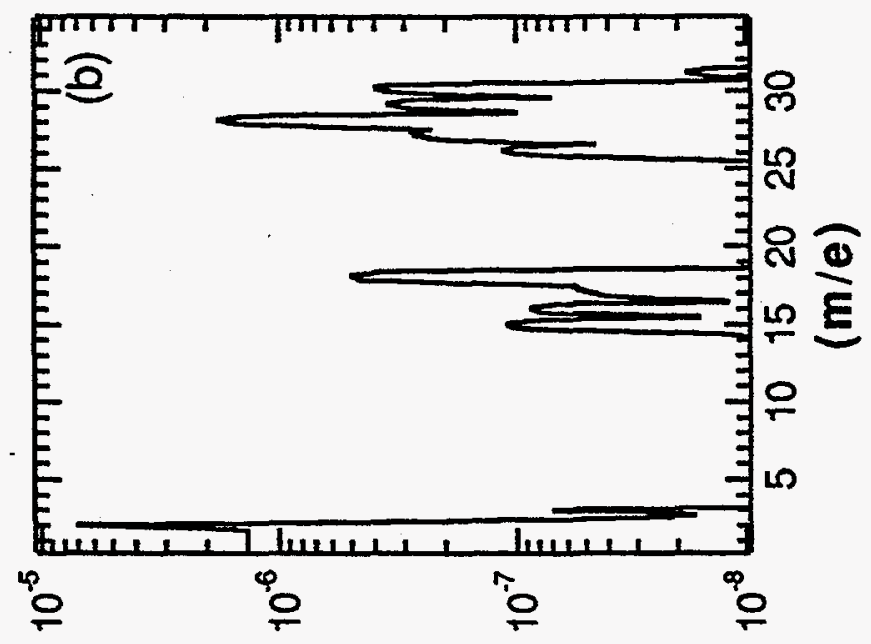

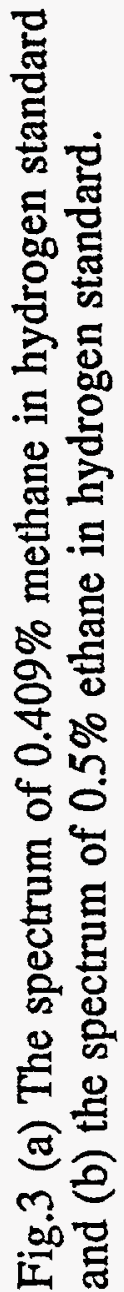

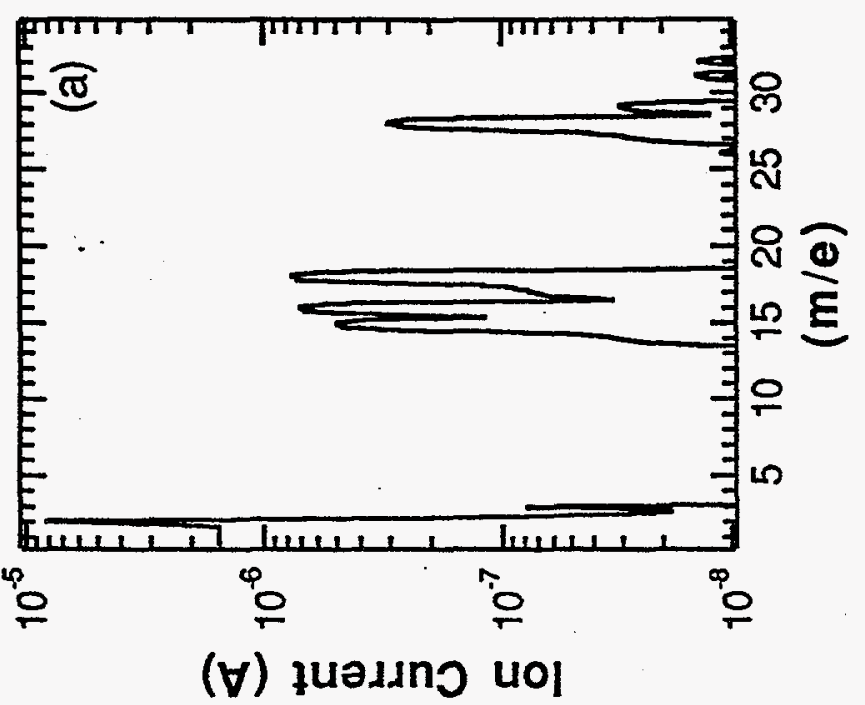


TMGa(normalized)

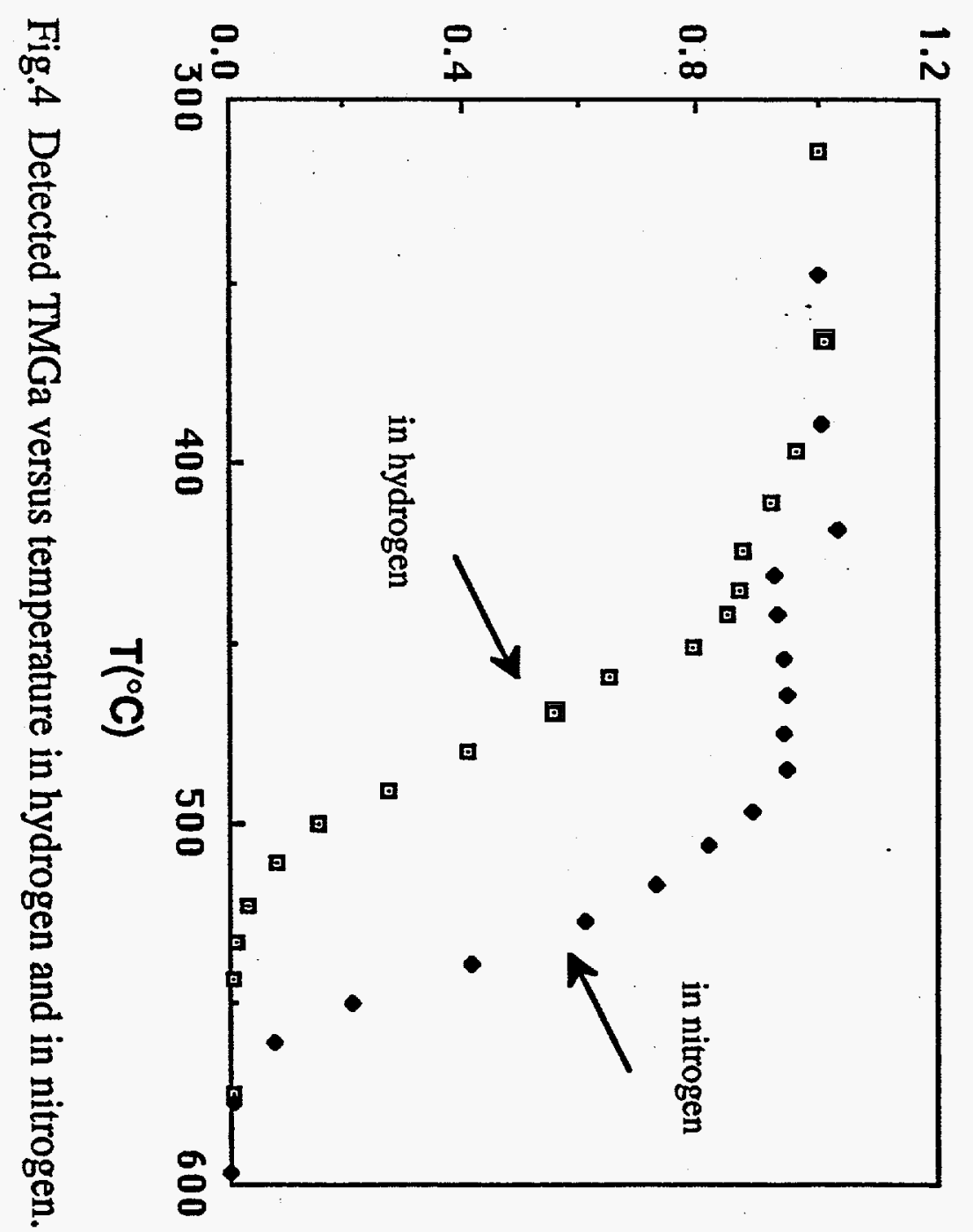




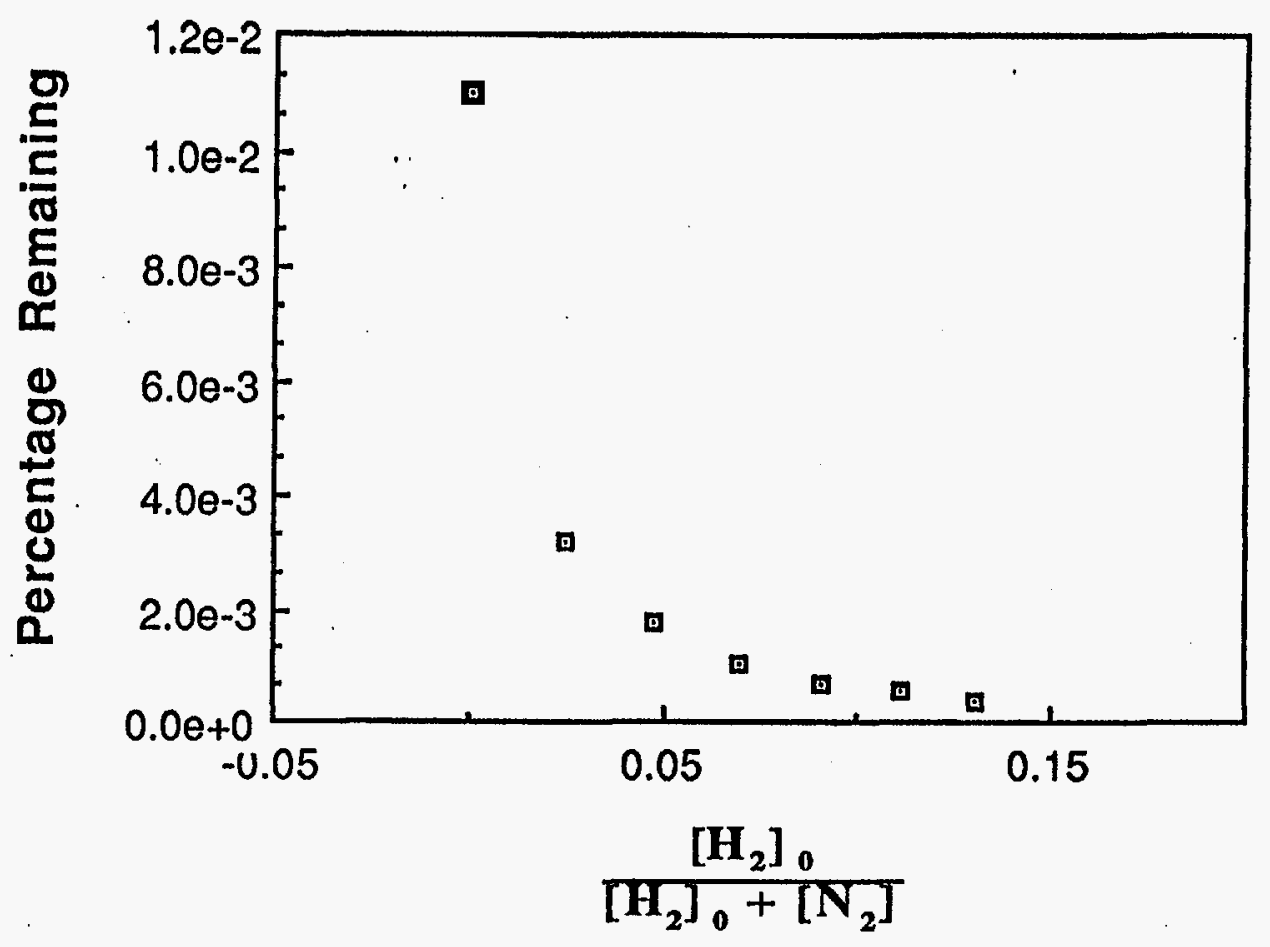

Fig.5 Remaining TMGa vs. input hydrogen mole fraction at $600{ }^{\circ} \mathrm{C}$. 


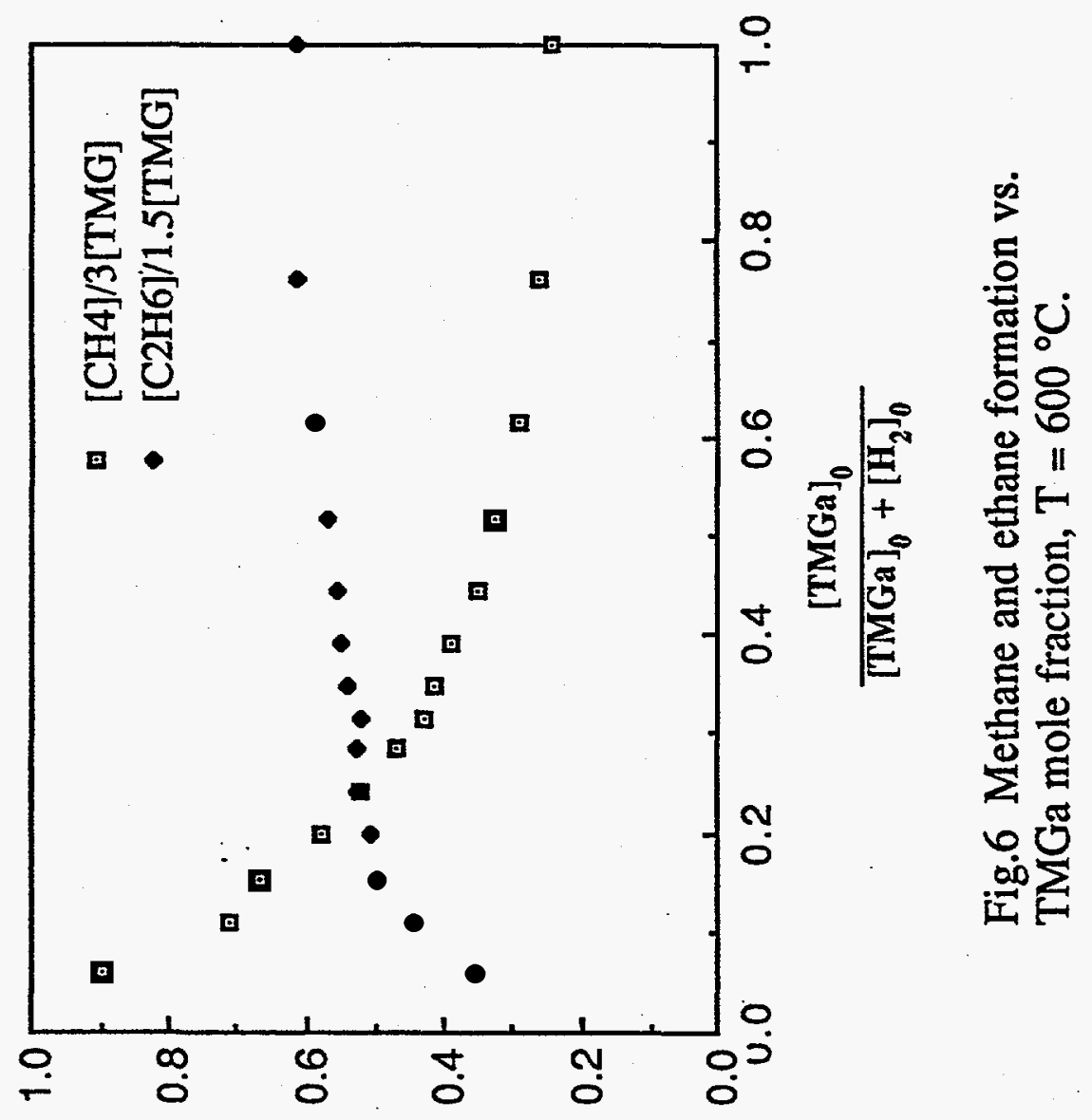




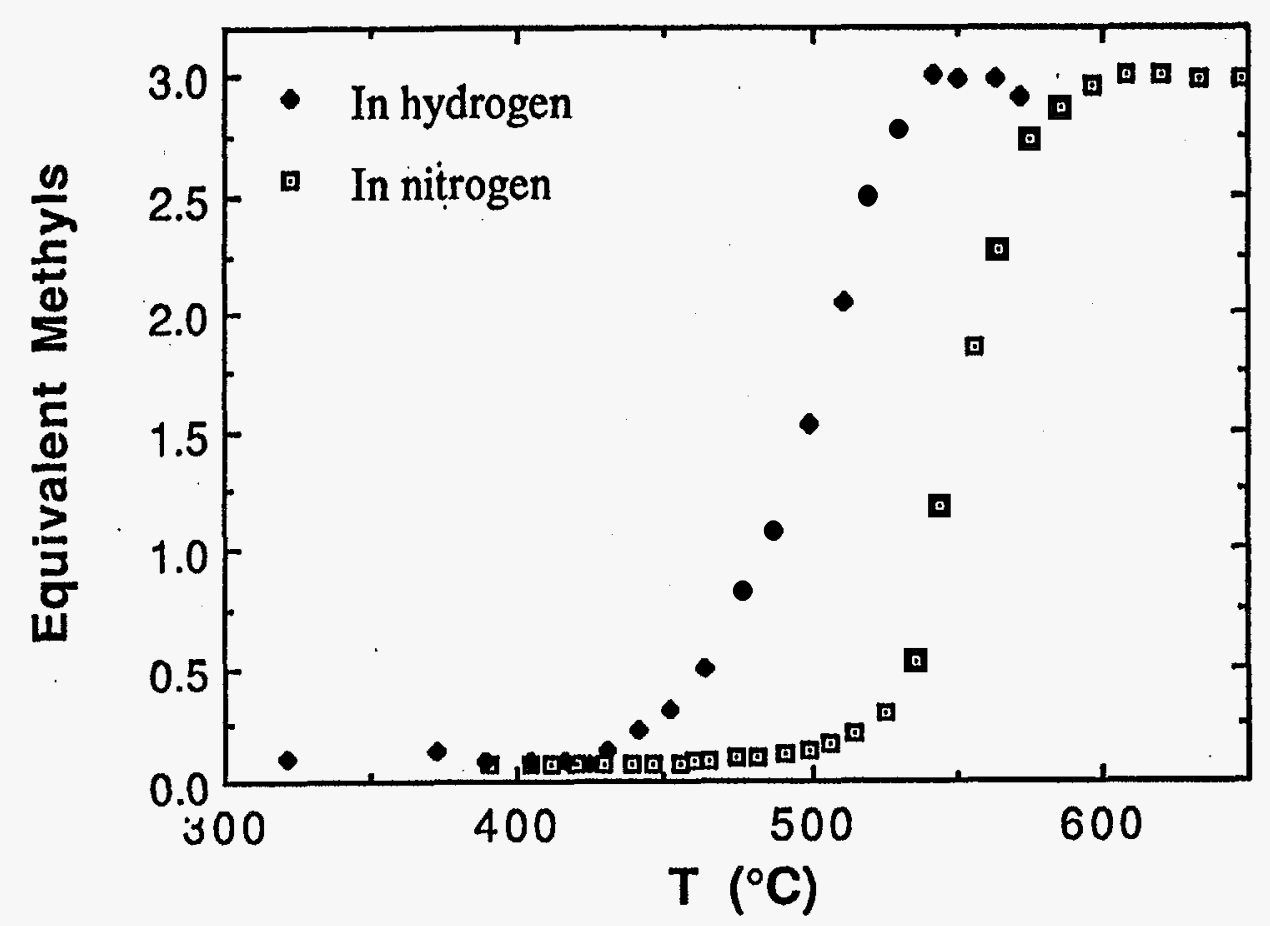

Fig. 7 Total low hydrocarbons in equivalence to the methyls in each mole of input TMGa. 


\section{References}

[1] M.G.Jacko and S.J.W.Price, Can. J. Chem., Vol.41, 1560, (1963).

[2] M.R.Leys and H.Veenvliet, J. Crystal Growth, Vol.55,145, (1981).

[3] J.Nishizawa and T.Kurabayashi, J. Electrochem. Soc., Vol.132(3), 413, (1983).

[4] S.P.DenBaars, B.Y.Maa, P.D.Dapkus, A.D.Danner, and H.C.Lee, J. Crystal Growth, Vol.77, 188, (1986).

[5] J.E.Butler, N.Bottka, R.S.Sillmon, and D.K.Gaskill, J. Crystal Growth, Vol.77, 163, (1986).

[6] D.Mazzarese, A.Tripathi, and W.C.Conner, J. Electron. Mat., Vol.18 (3), 369, (1989).

[7] M.Yoshida, H.Watanabee, and A.Uesugi, J. Electrochem. Soc., Vol.132(3), 677, (1985).

[8] P.W.Lee, T.R.Omstead, D.R.Mckenna, and K.F.Jensen, J. Crystal Growth, Vol.85, 165, (1987).

[9] N.Putz, H.Heinecke, M.Hegen, and P.Balk, J. Crystal Growth, Vol.74, 292,(1986).

[10] M.Mashita, S.Horiguchi, M.Shimizu, K.Kamon, M.Mihara, and M.Ishii, J. Crystal Growth, Vol.77, 194, (1986).

[11] C.A.Larsen, N.I.Buchan, and G.B.Stringfellow, Appl. Phys. Lett., Vol.52 (6), 480, (1988).

[12] H.Suzuki, K.Mori, M.Kawasaki, and H.Sato, J. Appl. Phys., Vol.64 (1), 371, (1988).

[13] M.A.A.Clyne and W.S.Nip, in "Reactive Intermediates in Gas Phase: Generation and Monitoring", D,W,Setser,Ed., Academic Press, (1979), p38.

[14] A.Kantrowitz and J.Grey, Rev. Sci. Instrum., Vol.22 (5), 328, (1951). .

[15] H.Parker, A.R.Kuhthau, R.Zapata, and J.E.Scott,Jr., in "Rarefied Gas Dynamics", F.M.Devienne, Ed., Pergamon Press, (1958), p69.

[16] S.W.Benson, "Thermochemical Kinetics: Methods for Estimation of Thermochemical Data and Rate Parameters", 2nd Ed., J.Wiley (1976).

[17] L.H.Long, Pure Appl. Chem., Vol.2, 61, (1961).

[18] D.G.Tuck, in "Comprehensive Organometallic Chemistry", Vol.1, G.Wilkinson, F.G.A.Stone, and E.W.Abel Ed., Pergamon Press (1982),p683.

[19] G.Pilcher and H.A.Skinner in "Chemistry of Metal-Carbon Bond", F.R.Hartley and S.Patai, Ed., J.Wiley (1982), chap.2, p43.

[20] M.Tirtowidjojo and R.Pollard, J. Crystal Growth, Vol.77, 200, (1986).

[21] F.Glockling and R.G.Strafford, J. Chem. Soc., (A), 1761, (1971). 
[22] G.C.Eltenton, J. Chem. Phys. , Vol.15(7), 455, (1947).

[23] F.P.Lossing and A.W.Tickner, J. Chem. Phys., Vol.20(5), 907, (1952).

[24] "Handbook of Chemistry and Physics", 68 TH, R.C.Weast Ed., CRC (1987-1988), E-84.

[25] M.Tsuda, S.Oikawa, M.Morishita, and M.Mashita, Jpn. J. Appl. Phys., Vol.26(5), L564, (1987).

[26] G.L.Pratt, "Gas Kinetics", J.Wiley, (1969), p159.

[27] P.C.Kobrinsky and P.D.Pacey, Can.J. Chem., Vol.52, 3655, (1974).

[28] G.L.Pratt and D.Roger, J. Chem., Soc., Faraday Trans. I, Vol.12, 2769, (1976).

[29] T.F.Kuech and E.Veuhoff, J. Crystal Growth, Vol.68, 148, (1984).

[30] G.Arens, H.Heineke, N.Putz, L.Luth, and P.Balk, J. Crystal Growth, Vol.76, 305, (1986). 


\section{Appendix: Activation Energies from Products}

(a) The formulation

The three methyls in a TMGa molecule, if released, will somehow form $\mathrm{CH}_{4}, \mathrm{C}_{2} \mathrm{H}_{6}$, and $\mathrm{C}_{2} \mathrm{H}_{4}$. They can readily be detected. Therefore, we consider the reactions (1a), (1b), and (1c) only. The rate equations are

$\frac{\mathrm{d}[\mathrm{TMGa}]}{\mathrm{dt}}=-\mathrm{k}_{1 \mathrm{a}}[\mathrm{TMGa}]$

$\frac{\mathrm{d}[\mathrm{DMGa}]}{\mathrm{dt}}=\mathrm{k}_{1 \mathrm{a}}[\mathrm{TMGa}]-\mathrm{k}_{1 \mathrm{~b}}[\mathrm{DMGa}]$

$\frac{\mathrm{d}[\mathrm{MMGa}]}{\mathrm{dt}}=\mathrm{k}_{1 \mathrm{~b}}[\mathrm{DMGa}]-\mathrm{k}_{1 \mathrm{c}}[\mathrm{MMGa}]$

$\frac{\mathrm{d}\left[\mathrm{CH}_{3}\right]}{\mathrm{dt}}=\mathrm{k}_{1 \mathrm{a}}[\mathrm{TMGa}]+\mathrm{k}_{1 \mathrm{~b}}[\mathrm{DMGa}]+\mathrm{k}_{1 \mathrm{c}}[\mathrm{MMGa}]$

Implementing the integrals and noting that $[\mathrm{TMGa}]=[\mathrm{TMGa}]_{0}$ at $\mathrm{t}=0$, where the $t$ is the total contact time, we obtain

$\frac{\left[\mathrm{CH}_{3}\right]}{[\mathrm{TMGa}]}=\mathrm{Y}_{1}+\mathrm{Y}_{2}+\mathrm{Y}_{3}$

where

$\mathrm{Y}_{1}=1-\mathrm{e}^{-\mathrm{k}_{1 \mathrm{a}}}$

$Y_{2}=\frac{k_{1 b}}{k_{1 b}-k_{1 a}}\left(1-e^{-k_{1 a} t}\right)-\frac{k_{1 a}}{k_{1 b}-k_{1 a}}\left(1-e^{-k_{1 b} t}\right)$

(VII),

and

$\mathrm{Y}_{3}=$

$\frac{k_{1 b} k_{1 c}}{\left(k_{1 b}-k_{1 a}\right)\left(k_{1 c}-k_{1 a}\right)}\left(1-e^{-k_{1 a} t}\right)-\frac{k_{1 a} k_{1 b}}{\left(k_{1 b}-k_{1 a}\right)\left(k_{1 c}-k_{1 a}\right)}\left(1-e^{-k_{1 c} t}\right)$ 
$-\frac{k_{1 a} k_{1 c}}{\left(k_{1 b}-k_{1 a}\right)\left(k_{1 c}-k_{1 b}\right)}\left(1-e^{-k_{1 b} t}\right)+\frac{k_{1 a} k_{1 b}}{\left(k_{1 b}-k_{1 a}\right)\left(k_{1 c}-k_{1 b}\right)}\left(1-e^{-k_{1 c} t}\right)$

(VIII)

account for the equivalent methyls from reactions (1a), (1b), and (1c) correspondingly.

(b) The estimation of the pre-exponential factors

Within the frame of the transition state theory of chemical kinetics, the unimolecular Ahhrenius pre-exponential parameter is related to the entropy change from the reacting molecules to their transition state, $\Delta S^{\ddagger}$. A statistical thermodynamics approach can be used to estimate the entropy change. We consider only the rotational and vibrational contributions. Actually, the vibrational contribution is already small. The structure of TMGa is planar and those of $\mathrm{DMGa}^{*}$ and $\mathrm{MMGa}^{*}$ are taken to be linear. Their corresponding transition states are taken to be more resembling to those of the expected products. The energized bond is assumed to elongate to about 2.8 times the original length[15]. The vibration modes concerning the Ga-C bonds are, according to Tuck [17], $v_{\mathrm{Ga}-\mathrm{Me} \text {,strech }}=521 \mathrm{~cm}^{-1}(\mathrm{sym})$, $570 \mathrm{~cm}^{-1}\left(\right.$ asym),$v_{\mathrm{Ga}-\mathrm{C} 3, \text { deform }}=163 \mathrm{~cm}^{-1}$, and $v_{\mathrm{Ga}-\mathrm{Me}, \text { rock }}=612,727$, $769 \mathrm{~cm}^{-1}$. 
3.0 Reaction Mechanisms in the Thermal Decomposition of Triethylarsenic and Triethylarsine

A paper published in the Journal of the Electrochemical Society by $S$. $P$. DenBaars, B. Y. Maa, P. D. Dapkus and A. Melas, J. Electrochem. Soc. 136, 2067 (1989). 
Reprinted from JoURNAL OF THE ECECTROCHEMICAI SOCIETY

Vol. 136, No. 7, July 1989

Printed in U.S.A.

Copyright 1989

\title{
Reaction Mechanisms in the Thermal Decomposition of Triethylarsenic and Diethylarsine
}

\author{
S. P. DenBaars, B. Y. Maa, P. D. Dapkus, and A. Melas' \\ Center for Photonic Technology, Department of Electrical Engineering and Materials Science, University of Southern \\ California, Los Angeles, California 90089-0483
}

\begin{abstract}
Studies of the reaction mechanisms and rates of decomposition of triethylarsenic (TEAs) and diethylarsine (DEAsH) were measured-by sampled gas infrared absorption spectroscopy. The thermal decomposition of TEAs in a hydrogen gas ambient was observed to occur predominantly by $\beta$-hydride elimination in which ethylene $\left(\mathrm{C}_{2} \mathrm{H}_{4}\right)$ and $\mathrm{DEAsH}$ are produced. An activation energy of $45 \mathrm{kcal} / \mathrm{mol}$ is measured for the pyrolysis of the first As-C bond in the TEAs decomposition reaction. The pyrolysis of DEAsH was observed to produce both ethane $\left(\mathrm{C}_{2} \mathrm{H}_{6}\right)$ and ethylene $\left(\mathrm{C}_{2} \mathrm{H}_{4}\right)$ as by-products and was found to go to completion at temperatures about $500^{\circ} \mathrm{C}$. The decomposition of DEAsH is concluded to be heterogeneous since the measured activation energy of $20.6 \mathrm{kcal} / \mathrm{mol}$ for the As-H bond pyrolysis is significantly lower than the As- $\mathrm{H}$ bond strength.
\end{abstract}

In the interest of reducing the safety hazards associated with arsine gas, development of high purity organometallic group $V$ sources would be of considerable benefit to growth of gallium arsenide (GaAs) by metalorganic chemical vapor deposition (MOCVD). The use of liquid organometallic arsenic sources would greatly reduce the toxic hazard and handling problems associated with high pressure arsine gas cylinders. The group $V$ hydrides also have slow decomposition rates and require high temperatures for pyrolysis. Thus, large excesses of arsine are required to obtain good epitaxial growth. In contrast, group V organometallics sources might potentially decompose at temperatures allowing a more stoichiometeric mixture in the gas phase at "normal" growth temperatures or they may allow reduced temperature growth to eliminate temperature driven diffusion of dopants. These benefits have led to the investigation of alternative arsenic sources to replace arsine (1-4). However, little is currently understood about the basic chemical reactions operative in the MOCVD growth of GaAs with these new organoarsenic sources.

In this paper we present data on the reaction mechanisms and rates of decomposition of the organometallic $V$ sources triethylarsenic (TEAs) and diethylarsine (DEAsH) in a hydrogen carrier gas. In addition, the catalytic reactivity of single crystal GaAs surfaces on the decomposition of the gaseous reactants is also studied.

\section{Experimental}

The thermal decomposition of TEAs and DEAsH was measured by sampled gas infrared absorption spectroscopy at a variety of temperatures. The relative concentration of the reactants was measured by sampling a portion of the gas stream at the exit end of a well-controlled hot zone as shown schematically in Fig. 1. Hydrogen carrier gas was passed at a flow rate of $600 \mathrm{~cm}^{3} / \mathrm{min}$ through a heated quartz tube, $5.0 \mathrm{~cm}$ in diam, which was inserted in a hot wall furnace with a confined and well-controlled hot zone $12.5 \mathrm{~cm}$ long. The source materials, obtained from Morton ThiokolCVD Incorporated, were kept in a temperature bath at $20^{\circ} \mathrm{C}$. Six GaAs $(100)$ wafers were inserted

'Present address: Morton Thiokol Incorporated, CVD Incorporated, Woburn, Massachusetts 01801 . into the reactor in such a way as to maximize the interaction of the wafer surfaces with the sampled gas stream before exiting the hot zone.

\section{Results and Discussion}

The infrared absorption spectra of $\mathrm{DEAsH} / \mathrm{H}_{2}$ and TEAs/ $\mathrm{H}_{2}$ mixtures sampled from a flowing gas stream have been examined in detail at various temperatures. The room temperature spectra of DEAsH and TEAs is shown in Fig. 2 and Fig. 3 , respectively. For our current experiments we have monitored the IR absorption peaks at $2980,2080,955$, and $585 \mathrm{~cm}^{-1}$, which correspond to the $\mathrm{C}-\mathrm{H}, \mathrm{As}-\mathrm{H}$ vibrational modes and the $\mathrm{C}=\vec{C}$, As- $\mathrm{C}$ stretching modes, respectively (5). It is of interest to note that the As-H vibrational mode in the DEAsH molecule is located at 2080 $\mathrm{cm}^{-1}$, which is red shifted with respect to its value of 2122 $\mathrm{cm}^{-1}$ in the arsine molecule. Based upon this observation we conclude that the appearance of an unknown peak at $2080 \mathrm{~cm}^{-1}$ previously observed in decomposition studies of the TMGa/AsH 3 system (6) is likely to be caused by the formation of an intermediate organoarsenic compound. These compounds may play a role in conventional MOVPE growth of GaAs.

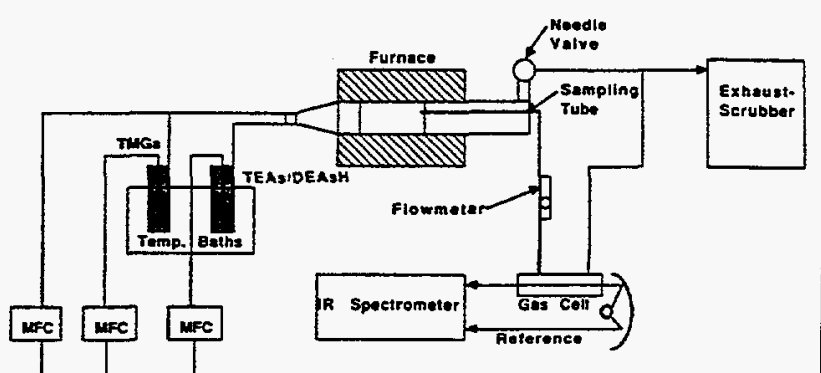

Mrarogen Puritler

Fig. 1. Experimental setup of MOCVD reaction kinetic studies by sompled gas infrored absorption spectroscopy. 


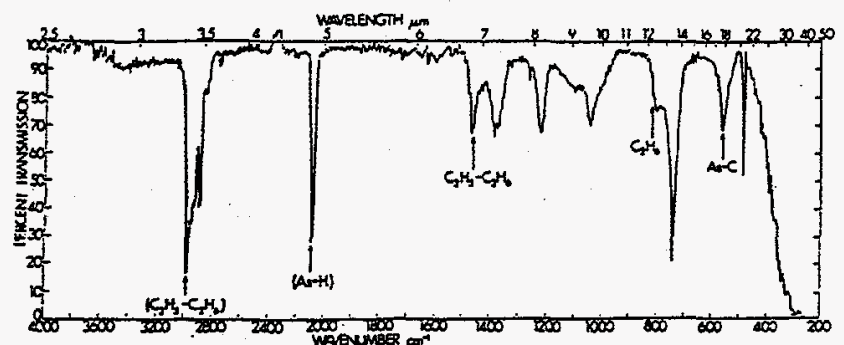

Fig. 2. Infrared absorption spectrum of diethylarsine at room temperature.

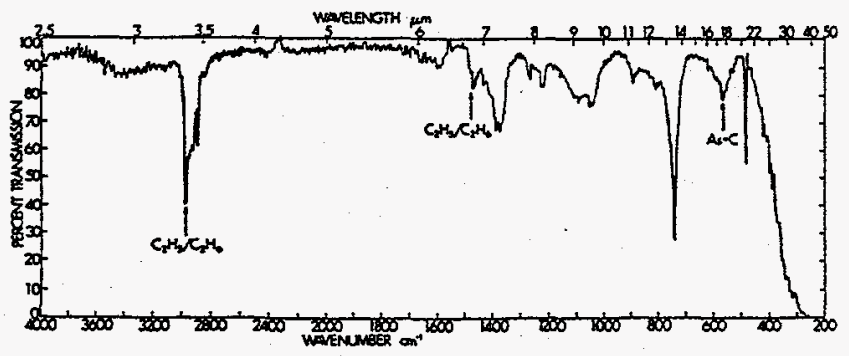

Fig. 3. Infrared absorption spectrum of triethylarsenic ot room temperature.

Upon thermally decomposing the DEAsH, ethane $\left(\mathrm{C}_{2} \mathrm{H}_{6}\right)$ and ethylene $\left(\mathrm{C}_{2} \mathrm{H}_{4}\right)$ are observed to be the main reaction products. In addition, a small quantity of arsine at 2122 $\mathrm{cm}^{-1}$ was observed to be produced at temperatures exceeding $440^{\circ} \mathrm{C}$. From infrared spectral analysis it was determined that the pyrolysis of DEAsH begins at $400^{\circ} \mathrm{C}$ and is virtually complete at $460^{\circ} \mathrm{C}$. In a hydrogen carrier stream the decomposition reaction for DEAsH appears to be

$$
\left(\mathrm{C}_{2} \mathrm{H}_{5}\right)_{2} \mathrm{AsH} \rightarrow 1 / 2 \mathrm{As}_{2}+(2-x) \mathrm{C}_{2} \mathrm{H}_{6}+x \mathrm{C}_{2} \mathrm{H}_{4}
$$

The value of $x$ for this reaction cannot be quantitatively determined since the absolute vapor pressure of $\mathrm{DEAsH}$ at $20^{\circ} \mathrm{C}$ is unknown.

Triethylarsenic shows a different reaction pathway, which interestingly enough yields DEAsH as an intermedate reaction product in the temperature range of $350^{\circ}$. $430^{\circ} \mathrm{C}$. Figure 4 shows an infrared absorption spectrum of TEAs taken at $410^{\circ} \mathrm{C}$, note the appearance of the As-H vibrational mode at $2080 \mathrm{~cm}^{-1}$. A $\beta$-hydride elimination mechanism is the most likely reaction pathway that explains the formation of DEAsH from the thermal decomposition of TEAs. This can be described by the following expression

$$
\left(\mathrm{C}_{2} \mathrm{H}_{5}\right)_{3} \mathrm{As} \rightarrow\left(\mathrm{C}_{2} \mathrm{H}_{5}\right)_{2} \mathrm{AsH}+\mathrm{C}_{2} \mathrm{H}_{4}\left(350^{\circ}-430^{\circ} \mathrm{C}\right)
$$

Figure 5 shows the relative concentration of the gaseous species as a function of temperature in the hot zone. Since the vapor pressure of DEAsH is not known, the concentration of DEAsH was estimated by taking the IR absorptivity at the As-H vibrational frequency to be one third of the value for the arsine molecule. Note that the evolution of DEAsH increases until a temperature of $430^{\circ} \mathrm{C}$ at which point the rate of decomposition of DEAsH exceeds the rate of its formation from the decomposition of TEAs. On ac-

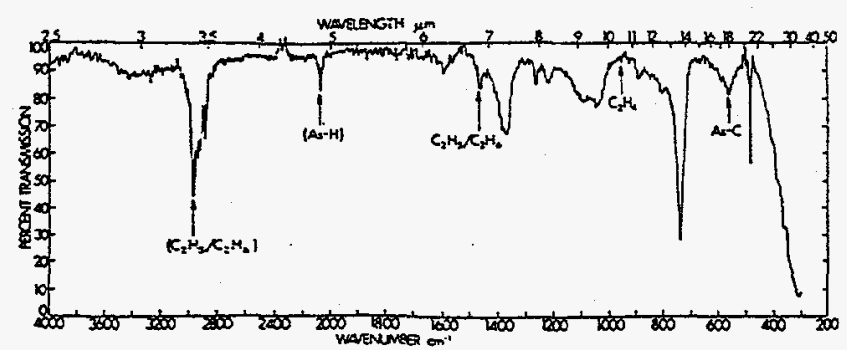

Fig. 4. Triethylorsenic spectrum at $410^{\circ} \mathrm{C}$, note oppearance of diethylorsine peck ot $2080 \mathrm{~cm}^{-1}$.

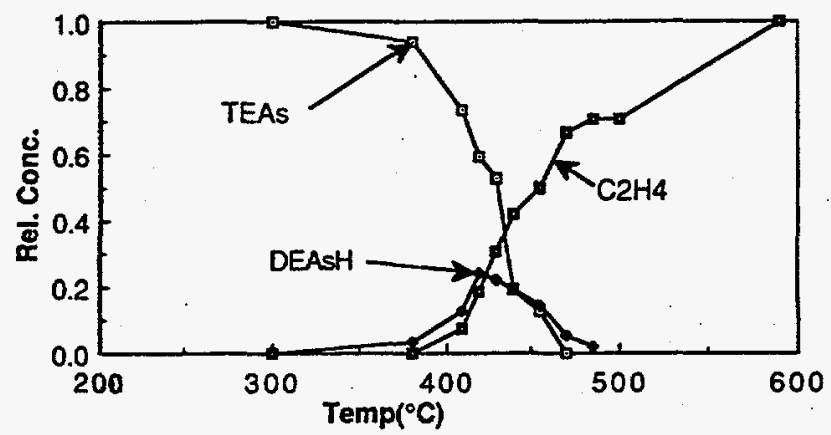

Fig. 5. Relative concentrotion of triethylarsenic, diethylarsine, and ethylese and os o function of temperoture.

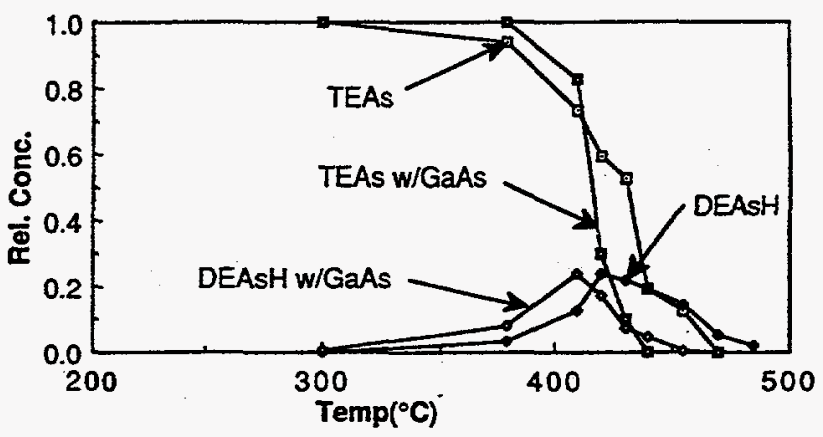

Fig. 6. Effect of GoAs $(100)$ surfoces on the pyrolysis of TEAs and DEAsH.

count of the overlap in the ethyl and ethane IR absorption peaks the relative concentration of $\mathrm{C}_{2} \mathrm{H}_{6}$ is not plotted.

The addition of GaAs(100) wafers to the hot zone has been found to catalyze the decomposition of both DEAsH and TEAs. As shown in Fig. 6 the relative rates of decomposition of both DEAsH and TEAs at $430^{\circ} \mathrm{C}$ are three times greater with GaAs surfaces in the hot zone. In addition, the slopes of the decomposition curves are steeper upon the addition of $\mathrm{GaAs}$ wafers which is indicative of a different activation energy for the process.

For the simple uncatalyzed pyrolysis of DEAsH and TEAs we model the kinetics of the decomposition by firstorder reaction kinetics as follows

$$
\frac{d C}{d t}=-k C
$$

where $C$ is the concentration of reactant, $t$ is reaction time, and $k$ is an effective rate constant of the form

$$
k=A \exp \left(-E_{A} / R T\right)
$$

The pre-exponential factor is the absolute rate and has units of $s^{-1}$ and $E_{\mathrm{A}}$ is the activation energy for the reaction. For a homogeneous unimolecular reaction, $E_{\mathrm{A}}$ is an approximate measure of the bond strength (7). The time and temperature dependence of the reactant concentration can be determined by rearranging Eq. [3] and integrating to yield the following expression

$$
C_{\varepsilon}=C_{0} \exp (-k \pi)
$$

where $\tau$ is the residence time of the gas in the hot zone, $C_{0}$ and $C_{r}$ are the initial and final reactant concentrations, $r e-$ spectively. This yields the following expression for the effective rate constant

$$
k=\ln \left(C_{d} / C_{f}\right) /(\tau)
$$

By using sampled gas infrared spectroscopy we have been able to measure the ratio of $C_{d} / C_{f}$ and control the contact time by adjusting the total gas flow rate. Therefore from Eq. [4] we can see that a logarithmic plot of $k$ vs. $1 /(R T)$ will yield the activation energy for the process under study. As shown in Fig. 7, an activation energy of $43.3 \mathrm{kcal} / \mathrm{mol}$ was measured for the decomposition rate of TEAs. This was 


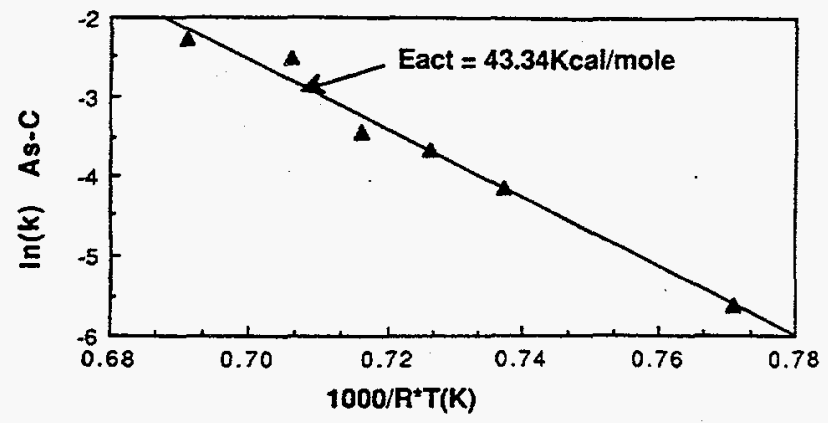

Fig. 7. Reaction rate of triethylarsenic plotted against inverse temperature times ideal gas constant yields activation energy of decomposition process.

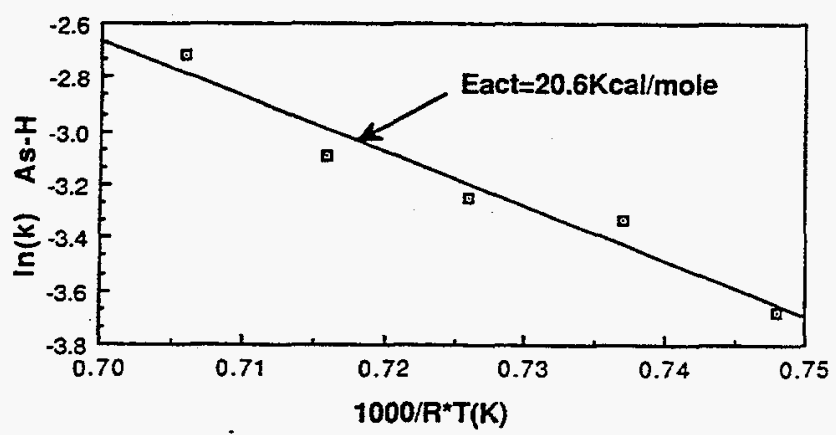

Fig. 8. Reaction rate of diethylarsine as a function of inverse temperature.

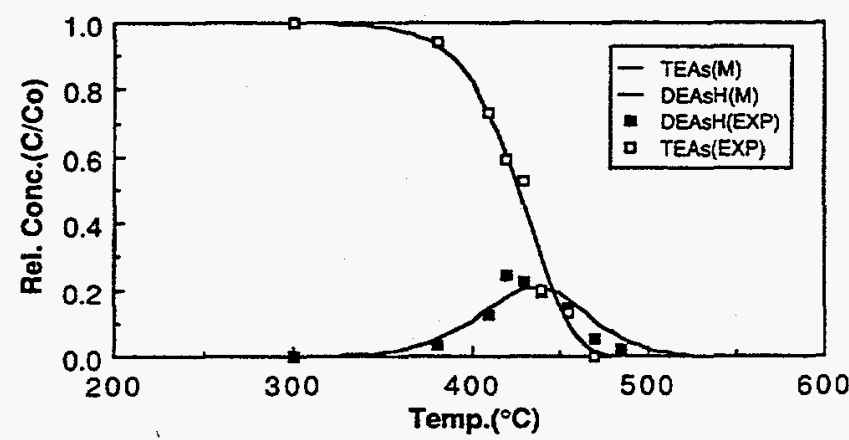

Fig. 9. Fit of reaction kinetics model to the experimentally observed relative concentrations of TEAs and DEAsH.

determined by monitoring the As- $\mathrm{C}$ stretching frequency $\left(565 \mathrm{~cm}^{-1}\right)$ in the TEAs molecule. The decomposition of DEAsH exhibited a significantly lower activation energy of $20.6 \mathrm{kcal} / \mathrm{mol}$ for the As- $\mathrm{H}$ vibrational frequency $\left(2080 \mathrm{~cm}^{-1}\right)$, see Fig. 8 . The activation energy for the decomposition of DEAsH is significantly lower than the As- $\mathrm{H}$ bond strengths (8) in $\mathrm{AsH}_{3}$, similar to results of earlier studies on the decomposition of $\mathrm{AsH}_{3}(9,10)$.

By using the effective reaction rate constants shown in Table I, we can produce a kinetic model which supports the experimentally observed evolution of $\mathrm{DEAsH}$ and $\mathrm{C}_{2} \mathrm{H}_{4}$ from the decomposition of TEAs by a $\beta$-hydride elimination mechanism. The temperature dependence of the relative concentration of $\operatorname{TEAs}\left(C_{f} / C_{0}\right)$ at a fixed contact time is shown in Fig. 9, in which both the line representing the kinetic model Eq. [7] and experimentally measured points are shown

$$
\frac{C_{f}}{C_{0}}(\text { TEAs })=\exp \left(-k_{1} \tau\right)
$$

Table I. Effective reaction rate constants

In $(k 1)$ TEAs $\left(s^{-1}\right)=27.8-(43,340 / R T)$

In $(k 2)$ DEASH $\left(\mathrm{s}^{-1}\right)=11.8-(20,624 / R T)$

If we assume that all the TEAs decomposes to form DEAsH then the relative amount evolved should be (1 $C_{f} / C_{0}$ ). By using the effective rate constant for the decomposition of DEAsH observed separately in the DEAsH experiments we can write the following kinetic expression for the final DEAsH concentration observed

$$
\frac{C_{\mathrm{f}}}{C_{\mathrm{o}}}(\mathrm{DEAsH})=\left[1-\exp \left(-k_{1} \tau\right)\right] \exp \left(-k_{2} \tau\right)
$$

As shown in Fig. 9, there is an excellent fit of the experimental data to the kinetic model. This supports the conclusion that the predominant mechanism for TEAs decomposition is $\beta$-hydride elimination resulting in the formation of DEAsH and ethylene. Consequently, it appears that DEAsH is the more stable organoarsenic molecule.

This is also supported by the experimental observation that upon the addition of trimethylgallium (TMGa) to gas stream a low volatility liquid adduct forms with TEAs, but not with DEAsH. The formation of the TEAs/TMGa adduct has required heating of reactor lines to $120^{\circ} \mathrm{C}$ in order to obtain successful GaAs growth (9). The use of DEAsH in MOCVD growth has yielded reasonably high purity GaAs with n-type background free carrier concentrations as low as $3 \times 10^{14} \mathrm{~cm}^{-3}$ and $77 \mathrm{~K}$ mobility of $64,600 \mathrm{~cm}^{2} /(\mathrm{V} \cdot \mathrm{s})$. On the basis of our studies and previous work indicates that DEAsH is a most promising arsine replacement. In addition, the As to hydrogen bond in DEAsH may play an important role in reducing the carbon incorporation in GaAs by hydrogenolysis of the methyl groups from TMGa. This view is supported by the work of Bhat (11) who observed high p-type background GaAs layers when elemental arsenic was substituted for arsine.

\section{Conclusions}

In conclusion, we have presented data which indicate that the thermal decomposition of TEAs in $\mathrm{H}_{2}$ occurs via a $\beta$-hydride elimination mechanism, producing DEAsH and $\mathrm{C}_{2} \mathrm{H}_{4}$ as the organic by-products. Thermal decomposition of DEAsH was observed to produce predominantly $\mathrm{C}_{2} \mathrm{H}_{6}$, $\mathrm{C}_{2} \mathrm{H}_{4}$, and a small quantity of arsine. Both DEAsH and TEAs decomposition was discovered to be catalyzed by the addition of single-crystal GaAs(100) surfaces to the reactor. Since DEAsH does not form an adduct with TMGa, whereas TEAs does, DEAsH is concluded to be the more attractive group $V$ alternative source.

Manuscript submitted April 4, 1988; revised manuscript received July $18,1988$.

Dr. S. P. DenBaars and Professor P. D. Dapkus assisted in meeting the publication costs of this article.

\section{REFERENCES}

1. P. Lee, D. McKenna, D. Kapur, and K. F. Jensen, J. Crystal Growth, 77, 120 (1986).

2. W. T. Tsang, Appl. Phys. Lett., 45, 1234 (1984).

3. R. Bhat, M. A. Koza, and B. J. Skromme, ibid., 50, 1194 (1987).

4. D. M. Speckman and J. P. Wendt, ibid., 50, 676 (1987).

5. M. R. Leys and H. Veenvliet, J. Crystal Growth, 55, 145 (1981).

6. J. Nishizawa and T. Kurabayashi, This Journal, 130, 413 (1983).

7. S. W. Benson, "Thermochemical Kinetics," 2nd ed, p. 86, John Wiley \& Sons, Inc., New York (1976).

8. S. R. Gunn, Inorg. Chem, 11, 796 (1972).

9. K. Tamaru, J. Phys. Chem., 59, 777 (1955).

10. S. P. DenBaars, B. Y. Maa, P.D. Dapkus, A. D. Danner, and H. C. Lee, J. Crystal Growth, 77, 188 (1986)

11. R. Bhat, J. Electron. Mater., 14, 433 (1985). 
4.0 Atomic Layer Epitaxy of III-V Compound Semiconductors by Thermal and Laser Assisted Metalorganic Chemical Vapor Deposition.

The Dissertation of Steven P. DenBaars in partial fulfillment of the requirements for the PhD at the University of Southern California. 
ATOMIC LAYER EPITAXY OF III-V COMPOUND

SEMICONDUCTORS BY THERMAL AND LASER-ASSISTED

METALORGANIC CHEMICAL VAPOR DEPOSITION

by

Steven P. DenBaars

A Dissertation Presented to the

FACULTY OF THE GRADUATE SCHOOL

UNIVERSITY OF SOUTHEIRN CALIFORNIA

In Partial Fulfillment of the

Requirements for the Degree

DOCTOR OF PHILISOPHY

(Electrical Engineering)

October 1988

Copyright 1988 Steven P. DenBaars 


\section{UNIVERSITY OF SOUTHERN CALIFORNIA THE GRADUATE SCHOOL \\ UNIVERSTTY PARK \\ LOS ANGELES. CALIFORNIA 90089}

This dissertation, written by

Steven P. DenBaars

under the direction of h.is........ Dissertation Committee, and approved by all its members, has been presented to and accepted by The Graduate School, in partial fulfillment of requirements for the degree of

DOCTOR OF PHILOSOPHY
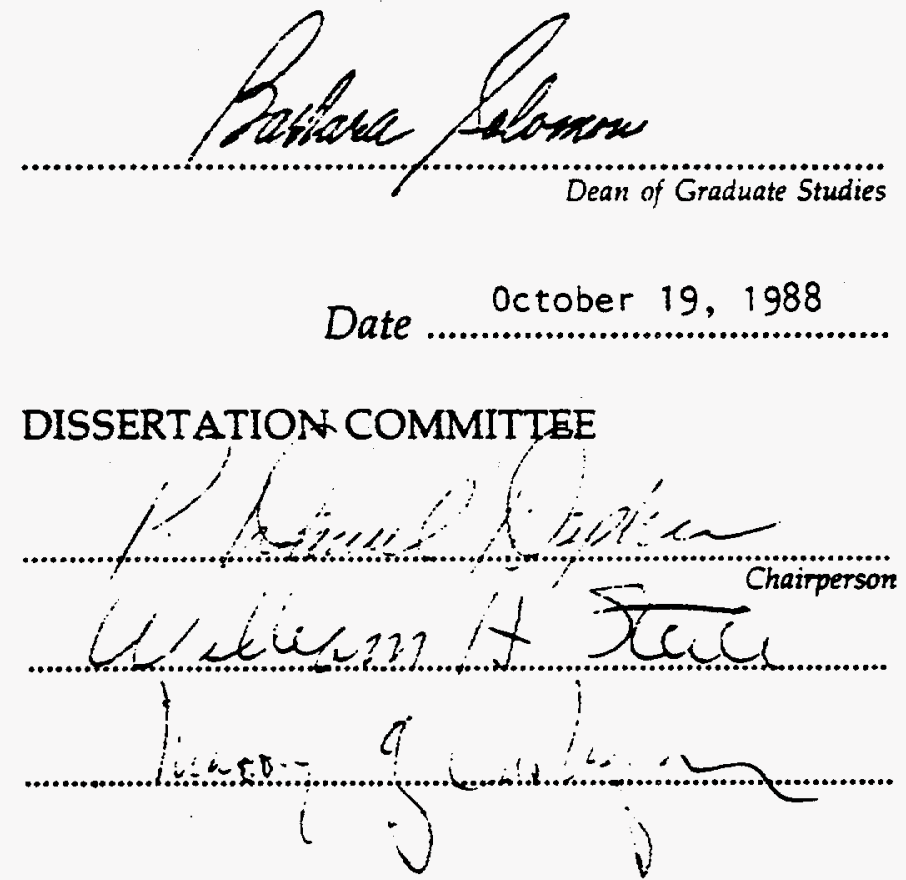


\section{DEDICATION}

I would like to dedicate this dissertation to my wife

Leslie, and to my parents for all the support and encouragement they provided. 


\section{ACKNOWLEDGEMENTS}

My deepest thanks are to my thesis advisor, Dr. P. Daniel Dapkus, for his constant support, inspiration, and ideas on the work presented here. Dr. Dapkus' enthusiastic attitude toward research has made graduate school an interesting and rewarding experience. I would also like to thank Dr. W. H. Steier and Dr. M. Gershenzon for serving as committee members and for the excellent classes they teach.

I am especially grateful to an excellent group of fellow graduate students. I would like to thank Dr. H. C. Lee and Dr. A. D. Danner for training me on the MOCVD reactor. For their support of my research efforts and friendship I would like to thank Chris Beyler, Qisheng Chen, Ken Dzurko, Ashan Hariz, Steve Hummel, Weon Jeong, Ben Maa, Dr. E. P. Menu, Jules Osinski, Dr. D. A. Sunderland, Majid Zandian, and Yao Zao.

I would like to thank my wife Leslie for all the support and encouragement. I would also like to acknowledge my parents and brothers for their support. 


\section{TABLE OF CONTENTS}

DEDICATION. ii

ACKNOWLEDGEMENTS. iii

LIST OF FIGURES. viii

LIST OF TABLES. xviii ABSTRACT. xix

1.0. INTRODUCTION .............................................. 1

1.1 Motivation.................................................... 1

1.2 Basic Theory of Atomic Layer Epitaxy...................... 6

1.2.1 Elemental Source ALE................................. 6

1.2.2 Molecular Source ALE................................ 7

1.3 Background Literature.......................................... 9

1.3.1 ALE of II-VI Compound Semiconductors............ 9

1.3.2 ALE of III-V Compound Semiconductors........... 12

1.3.3 Flow-rate Modulated Epitaxy.......................... 14

1.3.4 Laser Assisted ALE..................................... 15

1.4 Thesis Organization.......................................... 18

2.0 EPITAXIAL GROWTH BY MOCVD............................. 21

2.1 Introduction.................................................... 21

2.2 System Design................................................. 22

2.2.1 Source Material........................................... 24

2.2.2 Gas Handling.......................................... 25

2.2.3 Reactor Design......................................... 26

2.3 Basic Reaction Chemistry........................................ 26

2.3.1 Mass Transport Limited Regime...................... 29

2.3.2 Surface Kinetic Limited................................ 31

2.3.3 Desorption Limited..................................... 32

2.4 Hydrodynamic Concerns........................................ 32 
2.4.1 Boundary Layer Effects................................ 32

2.4.2 Compositional Control.................................. 35

2.5 Doping in MOCVD......................................... 36

2.5.1 p-Type Doping......................................... 36

2.5.2 n-Type Doping........................................ 37

2.6 Conclusions................................................. 38

3.0 REACTION KINETICS STUDY OF METALORGANIC CHEMICAL VAPOR DEPOSITION................................. 41

3.1 Introduction.................................................. 41

3.2 Reaction Kinetics............................................... 42

3.2.1 MOCVD Kinetic Steps................................... 42

3.2.2 Theory of Thermochemical Kinetics.................. 44

3.3 Experimental Setup........................................... 48

3.4 Sampled Gas Infrared Spectroscopy $\mathrm{TMGa}_{\mathrm{AsH}} / \mathrm{H}_{2} \ldots . . .51$

3.4.1 IR Spectra $\mathrm{TMGa} / \mathrm{AsH}_{3} / \mathrm{H}_{2}$........................... 51

3.4.2 Activation Energies................................... 59

3.5 Sampled Gas IR Spectroscopy DEAsH/TEAs .............. 66

3.5.1 IR Spectra DEAsH/TEAs............................ 67

3.5.2 DEAsH/TEAs Reaction Mechanism .................. 71

3.5.3 DEAsH/TEAsActivation Energies.................... 73

3.5.4 TEAS Decomposition Kinetics Model................. 75

3.6 Conclusions..................................................... 78

4.0 ATOMIC LAYER EPITAXY BY THERMALLY DRIVEN METALORGANIC CHEMICAL VAPOR DEPOSITION....... 82

4.1 Introduction................................................. 82

4.2 Design of ALE Reactor........................................ 83

4.3 Experimental Procedure........................................ 86

4.4. Growth and Characterization................................ 90

4.4.1 ALE Growth Results..................................... 90

4.4.2 Digital Growth Nature................................. 93

4.4.3 Thickness Uniformity................................... 94

4.4.4 Cleaved Corner TEM.................................... 98 
4.4.5 SEM Evaluation of ALE Layer Thicknesses......... 101

4.4.ú Morphology ALE GaAs................................ 101

4.4.7 Electrical Characterization............................ 105

4.4.8 Optical Properties........................................ 107

4.5 ALE Growth Model............................................ 109

4.5.1 Proposed ALE Reaction Model......................... 109

4.5.2 Calculated Surface Coverages.......................... 111

4.5.3 Kinetic Models of ALE Process......................... 114

4.5.3.1 ALE Growth Model 1......................... 114

4.5.3.2 ALE Growth Model 2........................ 118

4.5.3.3 Boundary Layer................................ 121

4.5.4 Kinetics of ALE in Nitrogen.......................... 122

4.6 Conclusions................................................. 125

5.0 ATOMIC LAYER EPITAXY OF AlGaAs/GaAs HETEROSTRUCTURES AND QUANTUM WELL LASERS.. 128

5.1 Introduction................................................. 128

5.2 Quantum Well Properties..................................... 130

5.2.1 Calculation of Eigenvalues in Finite Potential

Well....................................................... 130

5.3 ALE Quantum Wells......................................... 136

5.3.1 PL Linewidths......................................... 138

5.3.1.1 Interface Broadening........................ 141

5.3.1.2 Alloy Broadening.............................. 145

5.3.2 Optimization of ALE Quantum Well

Luminescence............................................. 148

5.3.3 AlGaAs/GaAs Heterostructures Grown Entirely by ALE.............................................. 152

5.4 Quantum Well Injection Lasers................................. 157

5.4.1 Density of States....................................... 157

5.4.2 Growth and Fabrication................................ 160

5.4.3 Device Results.......................................... 161

5.5 Conclusions................................................. 169 


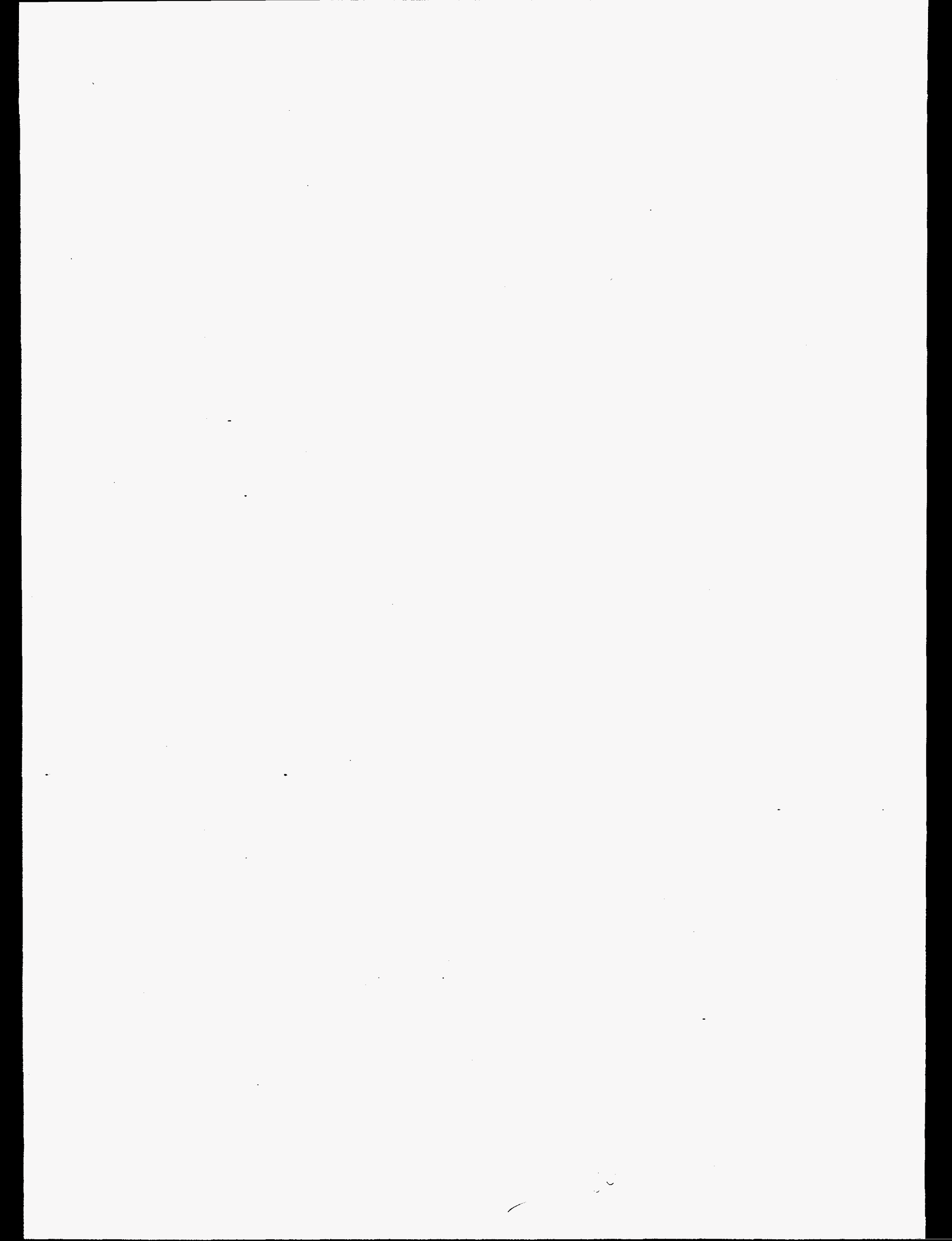




\section{LIST OF FIGURES}

Figure 1.1....

Film growth by the Elemental Source ALE method:(1) Excess incident $\mathrm{A}$ atoms evaporate, while $\mathrm{A}$ atoms that are bonded to underlying $B$ atoms form stable surface with small vapor pressure.(2) First layer of injected B atoms are chemisorbed to the surface while excess $B$ atoms evaporate.

Figure 1.2.

Method two (Gaseous Source ALE) utilizes surface exchange reactions between compound reactants: (1) one monolayer of AX gaseous precursor is chemisorbed on surface, excess AX molecules can only physisorb and evaporate off above a critical temperature, (2) reactor is purged with inert gas (H2), (3) precursor BY is injected and forms a complete monolayer by a surface exchange reaction, (4) reactor is purged in preparation for repeat of cycle.

Figure 1.3.

Dependence of growth rate per cycle on TEGa in the FME method.

Figure 1.4

Growth rate as a function of the laser power density in laserassisted ALE for both TMGa and TEGa.

Figure 1.5.

Laser-assisted ALE demonstrates a perfect monolayer per cycle growth rate over a wide range of TMGa fluxes. Thermally driven ALE shows saturated growth over a smaller range of conditions.

Figure 2.1

Schematic of conventional MOCVD system employed in this study.

Figure 2.2.

Growth rate versus reciprocal temperature for epitaxial layers on (100) and (111) Ga oriented GaAs substrates, from Reep and Ghandi. 
Figure 2.3

Growth rate as a function of $\mathrm{TMGa}$ flowrate shows linear dependence in the mass transport limited regime

$\left(\mathrm{T}_{\text {growth }}=750^{\circ} \mathrm{C}\right)$.

Figure 2.4

Schematic illustration of boundary layer in a horizontal MOCVD reactor. Note temperature and concentration profiles in the boundary layer (modified from Berkman et al.).

Figure 2.5

QW thickness versus wafer position on sample grown in conventional horizontal MOCVD reactor. Deposition was performed on a flat susceptor to exemplify the gas depletion effect.

Figure 2.6

Compositional control in MOCVD is achieved by linear adjustment of the molar fraction of the constituents. X-ray Diffraction determined composition data is from $\mathrm{H}$. C. Lee.

Figure 3.1

Schematic diagram illustrating some of the possible kinetic steps occurring in an MOCVD reactor.

Figure 3.2

Energy-reaction coordinate diagram for simple fission of molecule A-->B (after Benson).

Figure 3.3

Experimental Setup utilized in MOCVD reaction kinetic study. Note wafers inserted in well-controlled hot zone furnace.

Figure 3.4

GaAs wafers are arranged in rectangular quartz boat to insure maximum maximum interaction with sampled gas stream.

Figure 3.5

Room temperature IR spectrum of TMGa. 


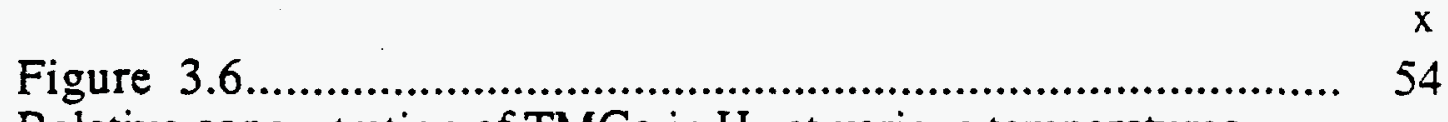

Relative concentration of $\mathrm{TMGa}$ in $\mathrm{H}_{2}$ at various temperatures.

Figure 3.7 .

55

Evolution of methane from pyrolysis of TMGa.

Figure 3.8

IR spectrum of $\mathrm{AsH} / \mathrm{TMGa} / \mathrm{H}_{2} \mathrm{MOCVD}$ system at $500^{\circ} \mathrm{C}$.

Figure 3.9

Relative concentration of $\mathrm{AsH}_{3}$ in $\mathrm{H}_{2}$ at various temperatures and for several different reactor conditions. Note the strong effect of the surfaces on $\mathrm{AsH}_{3}$ decomposition.

Figure 3.10

Effective decomposition rate constant versus reciprocal temperature for the decomposition of TMGa.

Figure 3.11

Ratio of evolved $\mathrm{CH}_{4}$ concentration to change in concentration of TMGa. The low temperature value of $R$ is 2.0 , while above $500^{\circ} \mathrm{C}$, $\mathrm{R} \sim 3$.

Figure 3.12

Effective rate constant for decomposition of arsine in $\mathrm{H}_{2}, \mathrm{H}_{2}+$ TMGa, and $\mathrm{H}_{2}$ with $\mathrm{GaAs}(100)$ wafers present.

Figure 3.13

68

Infrared absorption spectrum of diethylarsine at room temperature.

Figure 3.14

Infrared absorption spectrum of triethylarsenic at room temperature.

Figure 3.15

Triethylarsenic spectrum at $410^{\circ} \mathrm{C}$, note appearance of diethylarsine peak at $2080 \mathrm{~cm}^{-1}$. 
Figure 3.16

Relative concentration of triethylarsenic, diethylarsine and ethylene and as a function of temperature.

Figure 3.17

Effect of GaAs(100) surfaces on the pyrolysis of TEAs and DEAsH.

Figure 3.18

Reaction rate of triethylarsenic plotted against inverse temperature times ideal gas constant yields activation energy of decomposition process.

Figure 3.19 .

Reaction rate of diethylarsine as a function of inverse temperature.

Figure 3.20

Fit of reaction kinetics model to the experimentally observed relative concentrations of TEAs and DEAsH.

Figure 4.1

Schematic of fast gas switching ALE system. Note pressurebalancing of vent/run lines to minimize gas phase mixing of gaseous precursors.

Figure 4.2 .

Pulsed injection time sequence in four step ALE growth process.

Figure 4.3.

Hybrid ALE GaAs/AlGaAs single quantum well sample. Note $1 \mu \mathrm{m}$ thick AlGaAs cladding regions allow separation of PL from the individually grown ALE GaAs quantum wells

Figure 4.4

Comparison of growth rate of GaAs grown utilizing ALE growth regime and conventional MOCVD

Figure 4.5

Growth rate of ALE GaAs layers as a function of arsine injected into reactor. 
Figure 4.6.................................................................... 92

Growth rate of AlAs layers grown under ALE conditions as a function of TMAl injected into the reactor.

Figure 4.7 ...

Film thickness dependence on number of ALE deposition cycles demonstrates "digital" nature of ALE growth process.

Figure 4.8

$\mathrm{QW}$ thickness versus wafer position for quantum wells grown by conventional MOCVD and ALE.

Figure 4.9

The saturated growth mechanism operative in ALE makes the growth rate relatively insensitive to concentration fluctuations.

Figure 4.10 .

Cleaved-corner transmission electron micrograph of two ALE grown $\mathrm{GaAs}$ quantum wells (MAG $=1,200 \mathrm{kX}$ )

Figure 4.11

Schematic illustration of geometry of imaging conditions utilized in cleaved-comer TEM evaluation of GaAs/AlGaAs sample (modified from Kakibayashi et al.).

Figure 4.12.

SEM micrograph of 3 ALE AlAs layers with GaAs cladding regions. Each layer is grown with twice the flux of the layer preceding it. (MAG $=56 \mathrm{kX}$ )

Figure 4.13

Smooth surface morphology of $1 \mu \mathrm{m}$ thick GaAs grown by ALE at $460^{\circ} \mathrm{C}$.

Figure 4.14

Surface morphology of ALE layers grown above $500^{\circ} \mathrm{C}$ exhibit dendritic growth indicative of excess Ga. (SEM 5kX) 
Figure 4.15

Low temperature photoluminescence of ALE GaAs exhibits intense free exciton peak and donor to acceptor (carbon) peak.

Figure 4.16

Schematic illustration of the growth model showing Galliummethyl radicals chemisorbed on the surface. Layer growth is completed by the surface reaction of $\mathrm{AsH}_{3}$ with the gallium adsorbate.

Figure 4.17

Effect of temperature on growth rate in surface reaction controlled ALE growth regime.

Figure 4.18

First order model predicts self-limiting growth in the same temperature range as observed experimentally.

Figure 4.19

ALE growth rate as predicted by first order adsorption model with added deposition term accounting for decomposition of monomethylgallium to involatile gallium.

Figure 4.20

Monolayer coverage from arsine adsorption model.

Figure 4.21

Growth rate of ALE GaAs layers in both hydrogen and nitrogen carrier gas ambient as a function of TMGa injected.

Figure 4.22

Logarithmic plot of the cracking rate of TMGa in nitrogen and hydrogen (data from Yoshida et al.). Note the temperature difference necessary to obtain the same rate constant which corresponds to shift in ALE kinetic temperature range observed in Figure 4.21.

Figure 5.1

Energy band diagram for a GaAs/AlGaAs quantum well structure. 


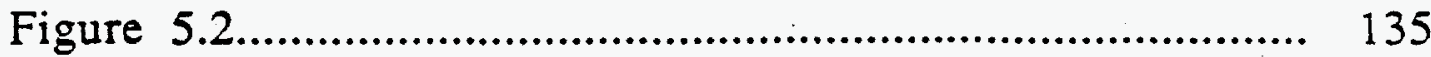

Shows the fit of experimental QW data to predicted emission wavelength.

Figure 5.3.

5 SQW sample grown for low temperature PL study.

Figure 5.4.

Low Temperature PL spectra of 5SQW exhibits narrow line intrinsic luminescence.

Figure 5.5

PL linewidth (FWHM) comparison of ALE and conventional grown MOCVD quantum wells.

Figure 5.6.

Monolayer variations in the width of small quantum wells result in shifts in emission energy.

Figure 5.7 .

Model of interface structure for interface island steps larger than the excitonic diameter (Case 1), and for interface islands steps smaller than excitonic diameter (Case 2 ).

Figure 5.8

Half-width of PL emission as a function of well width for one and two monolayer fluctuations. Lateral island size equals (a) $20 \AA$, (b) $80 \AA$, (c) $100 \AA$ (from Singh et al.). Solid line indicates one monolayer fluctuation, whereas dashed line indicates two monolayer fluctuation.

Figure 5.9

Local fluctuations in Aluminum concentration cause broadening of the PL emission.

Figure 5.10

The measured dependence of PL linewidth as a function of QW width for various growth methods. Solid lines indicate predicted PL broadening caused by fluctuations in $\mathrm{Al}$ composition with $33 \%$ Al barriers and $20 \AA$ lateral islands widths from S. B. Ogale et al. 
Figure 5.11

Room-temperature PL spectra of $5 \mathrm{SQWs}$ grown with varying surfaces coverages of arsine at $455^{\circ} \mathrm{C}$. Note that the insufficient arsine coverages reduce the PL efficiency and increase the PL FWHM linewidths.

Figure 5.12

Room-temperature PL spectra of 5 SQWs grown with varying surfaces coverages of $\mathrm{TMGa}$ at $455^{\circ} \mathrm{C}$.

Figure 5.13

PL spectrum of a GaAs/AlGaAs QW structure in which ALE was also used to grow the AlGaAs barrier layers.

Figure 5.14

PL spectra of a $70 \AA \mathrm{QW}$ with $\mathrm{Al} 0.2 \mathrm{Ga0} .8 \mathrm{As}$ ALE active region and $\mathrm{Al} 0.5 \mathrm{Ga} 0.5 \mathrm{As}$ barriers.

Figure 5.15

Low temperature PL spectra for five MQW structure in which 3 minute pauses at the AlGaAs interface lead to increased impurity incorporation. The uppermost spectrum shows narrow PL from QWs in which the pause was at the GaAs interface only. The lower spectrum shows the extrinsic broadened PL from QWs in which the pause was at the AlGaAs interface only.

Figure 5.16.

Quantum well laser offer discrete transitions energy levels because of the step-like 2-Dimensional density of states.

Figure 5.17

Schematic diagram of separate confinement $(\mathrm{SCH})$ heterostructure laser employing $60 \AA$ quantum well active region grown by ALE.

Figure 5.18

Spontaneous and laser emission spectrum from a. separate confinement quantum well laser with $\mathrm{GaAs} \mathrm{QW}$ active region by ALE. 
Figure 5.19

Mode spectrum of ALE SCH-QW injection laser showing longitudinal optical modes.

Figure 5.20

Energy Band diagrams of GRIN-SCH and SCH laser structures. Parabolic grading in GRIN-SCH creates pseudo-electric field and better optical confinement.

Figure 5.21

Luminescence intensity versus drive current for ALE QW injection laser

Figure 5.22

Top view of deposited $100 \mu \mathrm{m}$ wide $\mathrm{C}_{\mathrm{T}}$-Au contact stripe on $\mathrm{QW}$ injection laser. (MAG $=1000 \mathrm{X}$ )

Figure 6.1.

Photolytic deposition is achieved by excitation of molecular bonds by either (A) Multi-photon absorption, or (B) a single high energy photon. ( $E_{d}$ is the dissociation energy, $h v$ the photon energy)

Figure 6.2..

Schematic of four chamber reactor used in laser assisted ALE experiments. Note that the column III and column V reactants are separated spatially by two hydrogen purge channels and quartz baffles.

Figure 6.3

Laser assisted ALE exhibits self-limiting monolayer growth over a wide range of TMGa fluxes.

Figure 6.4

LALE deposits exhibit flat-top profile indicative of mono-atomic growth mechanism.

Figure 6.5

Photomicrograph of LALE GaAs stripe deposits exhibits smooth morphology of flat top stripes. (MAG $=100 X)$ 


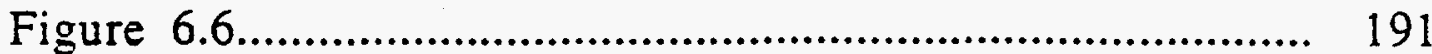

Low temperature PL from buried LALE CaAs stripes displays impurity related luminescence

Figure 6.7

UV illumination is observed to decrease the PL linewidth of thermally deposited ALE QWs.

Figure 6.8

Growth rate as a function of laser power for LALE of GaAs on Alo.3Gao.7As.

Figure 6.9

Temperature dependence of Thermal and Laser ALE. Note independence of LALE growth rate over $50^{\circ} \mathrm{C}$ range which is indicative of photocatalytic mechanism.

Figure 6.10.

Laser ALE model predicts saturated growth over a range of temperatures as was observed in experimental results. The model uses a $300 \mathrm{~mW}$ laser beam with a $40 \mu \mathrm{m}$ diameter scanned at $4000 \mu \mathrm{m} / \mathrm{sec}$.

Figure 6.11

Photocatalytic Model for catalytic chemisorption of gallium methyl molecules on a GaAs surface.

Figure 6.12

Calculated temperature rise for $50 \mu \mathrm{m}$ diameter laser beam at $200 \mathrm{~mW}$ scanned at $4000 \mu \mathrm{m} / \mathrm{sec}$.

Figure 7.1

LALE process using holographic projection for multiple device fabrication.

Figure 7.2

Multiple Wafer LALE deposition process. 


\section{ABSTRACT}

Atomic Layer Epitaxy (ALE) is a promising variation of conventional vapor phase epitaxy which achieves uniform growth of ultra-thin epitaxial layers by a self-limiting monolayer by monolayer deposition process. By employing a new regime of metalorganic chemical vapor deposition (MOCVD) growth, in which saturated surface reactions control the growth, it is possible to alternately deposit monolayers of column III and column V elements so that only one monolayer of the III-V compound semiconductor is deposited in every cycle of the deposition.

In this work, ALE growth of single crystal GaAs, as well as AlAs and GaAs/AlGaAs heterostructures and devices is demonstrated. We have been able to grow extremely uniform ultra-thin epitaxial layers and quantum wells (QWs) with thickness variations of less than one monolayer per $\mathrm{cm}$ over an entire sample in an optimized reactor using ALE. The observed dependence of the growth rate on temperature and trimethylgallium flux is modeled by first order adsorption kinetics utilizing measured reaction rate constants. The low temperature photoluminescence (PL) of ALE grown GaAs QW's exhibit narrow line intrinsic luminescence with linewidths comparable to the best reported values by conventional MOCVD. We have established for the first time that ALE can be hybridized with conventional MOCVD to grow device quality structures. This has been demonstrated by the operation for the first time of an injection laser with an active region grown by ALE. 


\section{LIST OF TABLES}

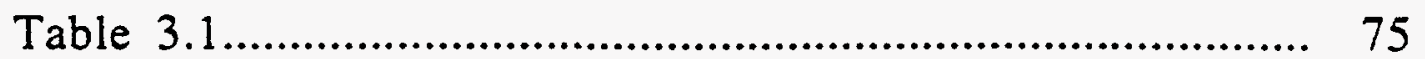

Rate constants for DEAsH/TEAs decomposition

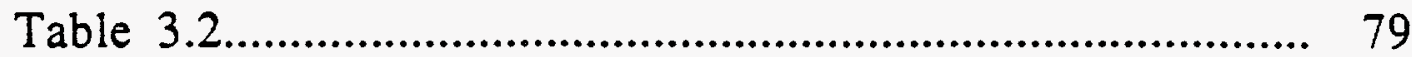

Rate constants for $\mathrm{TMGa}_{\mathrm{AsH}} 3$ decomposition.

Table 4.1.................................................................... 106

Electrical characteristics of ALE GaAs.

Table 4.2

Excess Surface Coverages $(\Theta)$

Table 4.3

Rate Constants

Table 5.1. 163

Threshold Current Densities

Table 6.1 198

Comparison of LALE techniques. 
Threshold densities as low as $380 \mathrm{~A} / \mathrm{cm}^{2}$ have been achieved for these structures.

Laser assisted ALE (LALE) of GaAs and related materials has been accomplished by the use of a Argon laser during the trimethylgallium (TMGa) exposure cycle in a novel four chamber ALE reactor. The "fast writing" potential of LALE to selectively deposit GaAs at scan speeds of $4000 \mu \mathrm{m} / \mathrm{sec}$ is demonstrated. We have employed a scanned laser beam with spot sizes as small as $30 \mu \mathrm{m}$ to deposit $\mathrm{GaAs}$ stripes on $\mathrm{Al} \mathrm{l}_{0.3 \mathrm{Ga}} \mathrm{G}$. As surfaces. Laser assisted ALE of GaAs displays several very favorable characteristics such as perfect monolayer saturated growth over an order of magnitude TMGa pressure, and growth saturation over a range of laser power densities. 


\section{CHAPTER 1 \\ INTRODUCTION TO ATOMIC LAYER EPITAXY}

\subsection{MOTIVATION}

In recent years, there has been an increasing demand for higher speed and higher efficiency operation of electronic and opto-electronic devices. The III-V compound semiconductor system is the leading materials system in which to produce the next generation of high speed electronic and photonic devices. Heterostructure devices composed of III-V semiconductors not only have a significant speed advantage over silicon based devices, 1 but also possess the ability to be integrated with optical devices on the same chip. In addition, heterostructure devices exhibit several interesting and useful physical phenomenon due to the quantum size effect which can be obtained in these structures. For such devices as high electron mobility transistors (HEMTs), quantum well lasers and other heterostructure devices, control of epitaxial layer thicknesses to within a single atomic layer becomes extremely desirable for high-yield implementation. Currently, metalorganic chemical vapor deposition (MOCVD) and molecular beam epitaxy (MBE) are the two leading processes utilized to grow these sophisticated semiconductor structures. Both processes have demonstrated sophisticated heterostructure devices with 1-2 monolayer abrupt interfaces in various III-V semiconductor systems. However, each technique has its own 
disadvantages and advantages.

Metalorganic chemical vapor deposition (MOCVD) is a nonequilibrium vapor phase growth technique which is typically operated at atmospheric pressure. The column III elements are transported to the growth reactor in the form of organometallic compounds, and the column $\mathrm{V}$ elements are supplied from gaseous hydride sources such as arsine and phosphine. The reactants are then simultaneously injected into the growth chamber where they thermally decompose in the hot boundary layer above the substrate and form single crystal layers. High quality materials and abrupt heterostructure devices have been grown by this technique. In addition, MOCVD shows the greatest promise for scale up to large area epitaxial growth for production of the next generation of electronic and opto-electronic devices owing to the economy and simplicity of the technique.

There are several inherent limitations of this growth technology. Because the growth rate is limited by diffusion of the column III reactant through the boundary layer, variations in layer thickness and compositional uniformity arise due to hydrodynamic fluctuations within the reactor. This dependence on hydrodynamics makes reactor design extremely crucial to both uniformity and scale. Currently, the best thickness uniformity achieved is $\pm 1.5 \%$ across 4 -cm diameter wafers in small research reactors. 2

The other leading technology for III-V semiconductor deposition is a high vacuum evaporation process known as molecular beam epitaxy (MBE). In this process shutters shield the crystal from molecular beams 
of the constituents and thin layers can then be grown by actuating these shutters. ${ }^{3}$ This technique has made impressive advances in demonstrating sophisticated heterostructure devices with extremely abrupt heterointerfaces of 1 monolayer. ${ }^{4}$ The main disadvantages of this technique has been the high cost of the equipment and it has proven to be extremely difficult and expensive to scale up this research technique to production capacities. 5

Utilizing these growth techniques to fabricate new heterostructure devices requires the precise control of such growth parameters as: growth time, flux of sources, and growth temperature. The ability to produce extremely uniform epitaxial layers is very important to the implementation of circuits based on HEMT's for which slight deviations in the channel thickness lead to significant threshold voltage variations. Quantum well injection laser threshold current and emission wavelength are also sensitive to well width. The sensitivity of these devices to the layer thickness and doping provides a major motivation for the subject of this thesis. In addition, development of a low temperature deposition process would result in reduction of defect concentration and elimination of temperature driven impurity redistribution.

In this dissertation, we have sought to develop a growth technology in which uniform large area deposition of one monolayer of column III elements followed by the deposition of one monolayer of column $\mathrm{V}$ elements is achieved. Atomic Layer Epitaxy (ALE) is a new crystal growth technique which allows control of the growth process at the atomic level.6.7 In contrast to conventional crystal growth, where the 
reactive gaseous precursors arrive simultaneously at the substrate, ALE proceeds by separately exposing the semiconductor surface such that one monolayer of an element containing adsorbate is deposited per exposure. Layer growth proceeds by repetition of this alternate exposure cycle. Because it employs saturated surface reactions, ALE is expected to produce extremely uniform layers and be amenable to the growth of ultrathin layers. Therefore, atomic layer epitaxy can be considered the crystal growth process with the ultimate in control because it operates with a self-limiting monolayer deposition mechanism at low substrate temperatures. For III-V compounds, the use of a volatile molecule for the column III element is required to avoid clustering of the element on the surface. In this work we have achieved ALE by employing conventional MOCVD reactants trimethylgallium (TMGa) and arsine $\left(\mathrm{AsH}_{3}\right)$ as the reactants for the ALE growth of GaAs.

The ALE technique has achieved excellent results in the II-VI compound semiconductor system, and is currently used in the manufacturing of electroluminescent displays. 8 ALE films can be grown by making use of either large pressure differences in the vapor pressures of the II-VI compounds and their pure constituents, or by sequential surface exchange reactions between compound reactants which have high vapor pressures in comparison with the product film.

The ALE process can also be stimulated locally by photolytically controlling the surface reactions with laser radiation. In this work we demonstrate that laser assisted ALE (LALE) selectively deposits GaAs with a perfect $100 \%$ monolayer saturated growth rate over a range of 
laser power densities and TMGa fluxes. We employ scan speeds of $4000 \mu \mathrm{m} / \mathrm{sec}$ to deposit $\mathrm{GaAs}$ stripes as small as $30 \mu \mathrm{m}$ on $\mathrm{Al}_{0.3 \mathrm{Ga}} \mathrm{G}_{7} \mathrm{As}$ surfaces. This "fast writing" aspect of LALE has great potential for integrating opto-electronic and electronic devices on the same chip. All of the above advantages result in ALE and LALE being attractive technologies for the growth of heterostructure devices with critical layer requirements. 


\subsection{Basic Theory of Atomic Layer Epitaxy}

\subsubsection{Elemental Source ALE}

Two different techniques have been discovered to yield atomic layer epitaxy in the II-VI semiconductor system. 9 The first technique utilizes elemental sources to achieve ALE, the second technique achieves monolayer growth from compound gas sources via a sequential surface exchange reactions. These two different methods are illustrated in figures 1 and 2. Method one (Elemental Source ALE) achieves ALE growth by making use of the large vapor pressure differences of the II-VI compound semiconductor compounds and their pure elemental constituents. Because of the low vapor pressure of column III metals at temperatures at which both the III-V bond and column V compounds are stable, self-limiting growth of III-V compounds is not thermodynamically possible using the elemental source method.

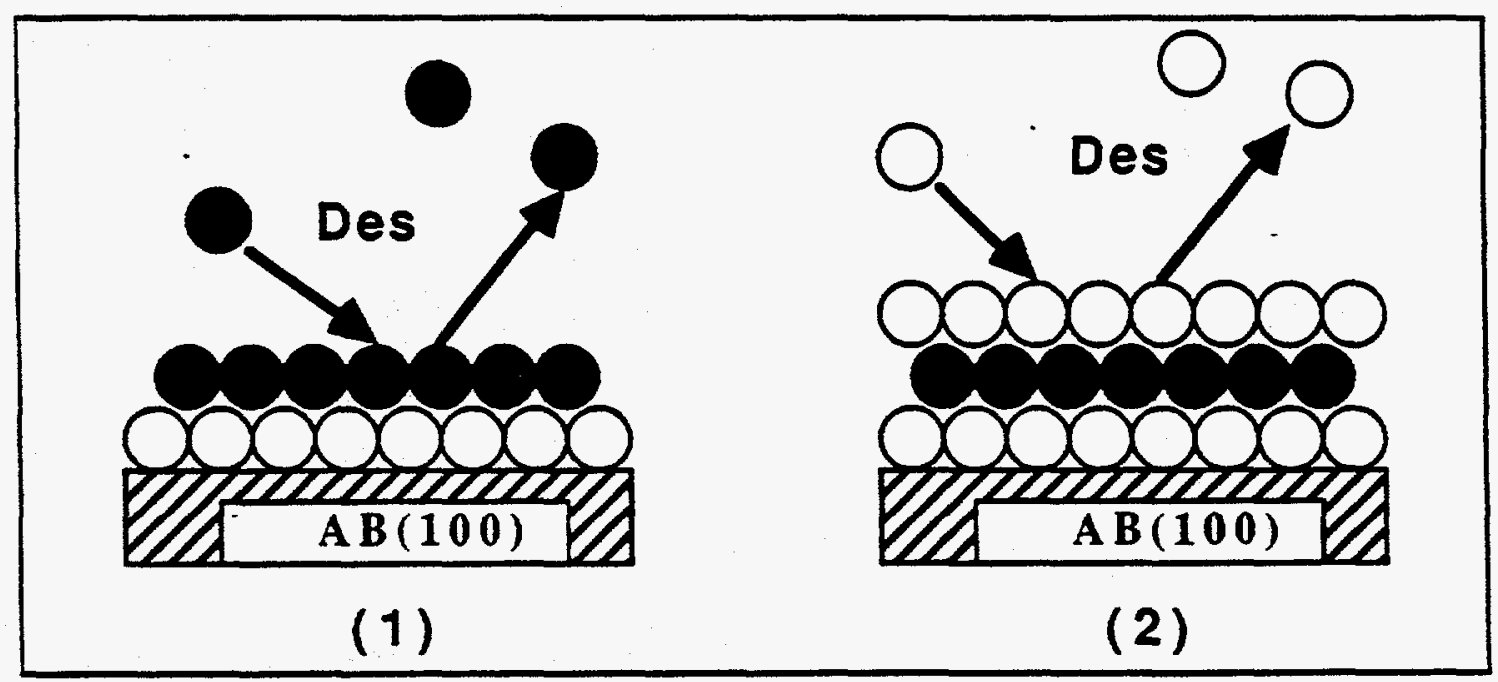

Figure 1.1

Film growth by the Elemental Source ALE method:(1) Excess incident A atoms evaporate, while $A$ atoms that are bonded to underlying $B$ atoms form stable surface with small vapor pressure.(2) First layer of injected B atoms are chemisorbed to the surface while excess B atoms evaporate. 


\subsubsection{Molecular Source ALE}

Method two (Molecular Source ALE) utilizes surface exchange reactions between compound reactants which have high vapor pressures in comparison to the product film. This technique achieves self-limiting growth which can be attributed to the fact that the first gaseous layer interacts strongly with the substrate (chemisorption), and subsequent layers interact less strongly (physisorption), and will evaporate above a critical temperature. Thus by obtaining a monolayer of chemisorbed compound reactant a self-limiting crystal growth process can be obtain. The general reaction for ALE by a surface exchange reaction can be expressed as follows:

$$
\begin{gathered}
A X_{(v)} \rightarrow A_{(\text {ads })} \\
A X_{(\text {ads })}+B Y_{(v)} \rightarrow A B_{(s)}+X Y
\end{gathered}
$$

For a self-limiting monolayer deposition process the time dependence of fractional surface coverage will be a function of both the rate of chemisorption on the unoccupied sites and the rate of desorption from the occupied sites. By using the following first order adsorption expression we can effectively describe the the ALE growth rate:

$$
\frac{d \theta}{d t}=k_{\text {ads }}(1-\theta)-k_{\text {des }}(\theta)
$$

Where: $\quad \Theta$ =fraction of occupied surface sites

$\mathrm{k}_{\mathrm{ads}}=$ rate of impinging molecules adsorbed on surface $\mathrm{k}_{\mathrm{des}}=$ desorption rate of molecules from the occupied sites 


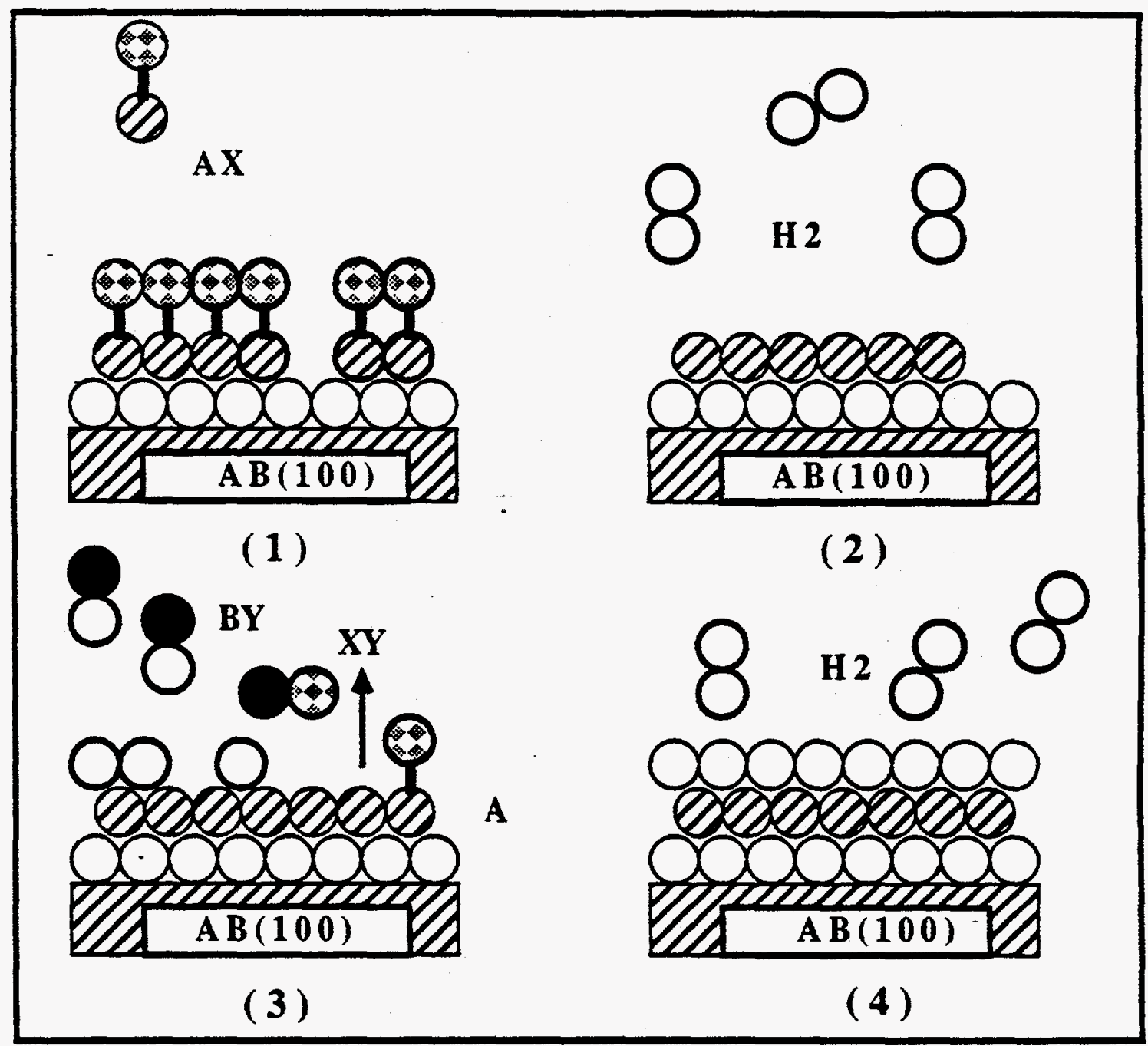

Figure 1.2

Method two (Gaseous Source ALE) utilizes surface exchange reactions between compound reactants: (1) one monolayer of AX gaseous precursor is chemisorbed on surface, excess AX molecules can only physisorb and evaporate off above a critical temperature, (2) reactor is purged with inert gas (H2), (3) precursor BY is injected and forms a complete monolayer by a surface exchange reaction, (4) reactor is purged in preparation for repeat of cycle. 


\subsection{Background Literature}

\subsubsection{ALE of II-VI Compound Semiconductors}

The first research on ALE was done in the II-VI semiconductor system. The most widespread commercial application of ALE has been the growth of $\mathrm{ZnS}$ for use in $\mathrm{AC}$ electroluminescent display panels. Both amorphous and polycrystalline $\mathrm{ZnS}$ has been grown by using both types of ALE deposition techniques. $\mathrm{ZnCl}_{2}$ and $\mathrm{Zn}\left(\mathrm{CH}_{3} \mathrm{COO}\right)_{2}$ in conjunction with $\mathrm{H}_{2} \mathrm{~S}$ have been utilized to achieve ALE of $\mathrm{ZnS}$ in a chemical vapor deposition (CVD) system. After exposing the surfaces to $\mathrm{ZnCl}$, films were analyzed by Auger Electron Spectroscopy (AES) which identified chemisorped $\mathrm{ZnCl}_{2}$. Surfaces were then exposed to $\mathrm{H}_{2} \mathrm{~S}$, after which subsequent analysis revealed only a small amount of $\mathrm{Cl}$. This indicates close to $100 \%$ completion of a surface exchange reaction. Reactions which govern the process are:

$$
\begin{gathered}
\mathrm{ZnCl}_{2(\mathrm{v})}---->\mathrm{ZnCl}_{2 \text { (chemisorped) }} \quad\left\{350^{\circ} \mathrm{C}-550^{\circ} \mathrm{C}\right\} \\
\mathrm{ZnCl}_{2 \text { (chemisorped) }}+\mathrm{H}_{2} \mathrm{~S}_{(\mathrm{v})}-\rightarrow \mathrm{ZnS}_{(\mathrm{s})}+2 \mathrm{HCl} \\
\mathrm{Zn}\left(\mathrm{CH}_{3} \mathrm{COO}\right)_{2}+\mathrm{H}_{2} \mathrm{~S} \rightarrow \mathrm{ZnS}+2 \mathrm{CH}_{3} \mathrm{COOH}
\end{gathered}
$$

Vacuum evaporation techniques utilizing elemental $\mathrm{Zn}$ and $\mathrm{S}$ 
sources have also been successful in obtaining films of $\mathrm{ZnS}$ by ALE. The crystalline quality of the $\mathrm{ZnS}$ is found to be highly dependent on the growth temperature and the type of substrate. 10 In general the degree of crystalline perfection was found to increase with increasing temperature. Both cubic and hexagonal structure was found in the deposited films. The dislocation density of $\mathrm{ZnS}$ ALE films $\left(1010 \mathrm{~cm}^{-2}\right)^{11}$ was found to be significantly lower than electron beam evaporated films $\left(1011 \mathrm{~cm}^{-2}\right)$.

Single crystal CdTe has been grown on $\mathrm{CdTe}(111), \mathrm{CdTe}(110)$ and GaAs(100) substrates. M. A. Herman et al. 12 have also utilized ALE to deposit CdTe films on $\mathrm{CdTe}(111) \mathrm{B}$ substrates. Using the elemental source method they observed monolayer depositions that were highly dependent on the pause time separating the $\mathrm{Cd}$ and $\mathrm{Te}$ pulses. This was achieved in a high vacuum evaporation system with elemental $\mathrm{Te}$ and $\mathrm{Cd}$ sources. Elemental sources can be used here since both $\mathrm{Cd}$ and $\mathrm{Te}$ have relatively large vapor pressures in comparison to the single crystal CdTe at growth temperatures of $270^{\circ} \mathrm{C}$. In this study it was determined crucial to accurately control the growth parameters since $\mathrm{Cd}$ and $\mathrm{Te}$ continue to re-evaporate and will form less then unity monolayer coverage. Therefore minimum pause durations of 0.5 seconds were found necessary to achieve good surface coverage. Typical growth conditions consisted of 1.0 second pulse of $C d$ and 2.5-2.8 second pulses of Te separated by 0.5 second delay times. The purge time $(\Delta \tau)$ between $\mathrm{Cd}$ and $\mathrm{Te}$ pulses was shortened to achieve monolayer by monolayer deposition. This observed growth behavior could be explained by taking into account the temperature dependence of the evaporation rate relative to lower 
temperatures with the following Arrhenius expression:

$$
R\left(T_{1}\right)=R\left(T_{0}\right) \exp \left[\frac{E_{a}\left(\frac{1}{T_{0}}-\frac{1}{T_{1}}\right)}{k}\right]
$$

The fractional monolayer coverage " $\Theta$ " of CdTe can than be modeled by taken into account this evaporation rate and balancing it with the incoming flux of $\mathrm{Cd}$ atoms. This can be expressed by the following equation:

$$
\theta=\frac{\left(\Phi_{C d} \tau-R_{C d} \Delta \tau\right)}{D_{C d}}
$$

Where: $\quad R_{C d}=$ Rate of Cd evaporating

$\mathrm{D}_{\mathrm{Cd}}=$ Surface density of $\mathrm{Cd}$ needed for one monolayer

$\Phi_{C d}=$ Incoming flux of $\mathrm{Cd}$ atoms

$\tau_{\text {exp }}=$ Exposure time

$\Delta \tau=$ Purge time 


\subsubsection{ALE of III-V Compound Semiconductors}

The first report of ALE in the III-V semiconductor system was reported by Nishizawa and co-workers in 1985.13 Utilizing gaseous sources in a high vacuum MBE apparatus, Nishizawa observed saturated monolayer growth of GaAs. Monolayer film growth was obtained at relatively low substrate temperatures $\left(450-500^{\circ} \mathrm{C}\right)$. A slow deposition rate of one atomic layer every 24 seconds was observed due to the inefficient cracking of the arsine in the high vacuum environment, thus comparatively long exposures of arsine are necessary. The deposited films were found to be heavily p-type with doping on the order of 1018 $\mathrm{cm}^{-3}$ and room temperature mobilities of around $100 \mathrm{~cm}^{2} / \mathrm{V} \cdot \mathrm{sec}$. Increasing the amount arsine injected into the reactor was found to substantially reduce the background doping, therefore improvements in the film quality are anticipated with optimization of the surface reaction of arsine. The addition of UV irradiation on the sample allowed singlecrystal growth to occur at substrate temperatures in the range of $350^{\circ} \mathrm{C}$. This is thought to be caused by either a photochemical effect or the fact that the migration length of admolecules is increased by photoirradiation as is the case for silicon epitaxy. 14

ALE of GaAs using hydride VPE was reported by Usi et al.15 Using a dual chamber growth system the substrate was alternately rotated between the $\mathrm{GaCl}$ flow and $\mathrm{AsH}_{3}$. Monolayer saturated growth was observed over an order of magnitude of $\mathrm{GaCl}$ pressures. This is in contrast to the linear dependence of growth rate on $\mathrm{GaCl}$ pressure usually 
observed in conventional VPE. Due to the long substrate transfer time the deposition rate was 60 seconds long for each monolayer. ALE was observed to occur over a wide range of temperatures with the hydride technique. Below $550^{\circ} \mathrm{C}$ all the deposited films were single crystal with good morphology and remarkably free of surface defects. No enhancement of layer thickness near the edge of windows opened in $\mathrm{SiO}_{2}$ was observed in this ALE technique. Large thickness variations have been a problem in conventional VPE where the growth is diffusion limited, thus excess reactants diffusing across the $\mathrm{SiO}_{2}$ mask enhance the growth rate near the edges of the window.

The first report of ALE in a atmospheric MOCVD reactor was by Bedair and Tischler 16 at North Carolina State University. The experimental setup consisted of rotating a susceptor between separated column III and column V gas streams. After exposure to each gas stream the recessed wafer is rotated past a fixed graphite edge assembly which effectively "shears the boundary layer," thus preventing any cross-mixing of the column III and column V gas stream. Saturated monolayer growth was observed at higher growth temperatures $\left(560-650^{\circ} \mathrm{C}\right)$ than was observed by both Nishizawa $12\left(460-500^{\circ} \mathrm{C}\right)$ and in the work reported here $\left(445-500^{\circ} \mathrm{C}\right)$. Photoluminescence of ALE GaAs sandwiched between GaAs $0.67 \mathrm{P}_{0.33}$ at $77 \mathrm{~K}$ exhibited an intense band to band peak with a 11 meV linewidth. Tischler et al. ${ }^{17}$ reported on ALE growth of InAs/GaAs single quantum well structures where the wells were only 2 and 4 monolayers thick. Photoluminescence from the quantum wells was found to be sharp, intense, and uniform across the sample with full widths at 
half-maximum for the 2 and 4 monolayer wells of 12 and $17 \mathrm{meV}$, respectively.

\subsubsection{Flow-rate Modulation Epitaxy}

A variation of ALE is a process entitled Flow-rate Modulation Epitaxy (FME) which was reported by Kobayashi et al. in 1985.18 This method is also based on alternating the gas flow of the column III and column V sources, however, a small amount of arsine is always injected into the reactor to suppress arsenic vacancies near the growing interface. To date, these workers have utilized triethylgallium (TEGa) for the column III gas source because it has a lower cracking temperature than TMGa. 19 In contrast to the p-type GaAs films previously reported in ALE films, the FME layers grown at $550^{\circ} \mathrm{C}$ were found to be n-type with carrier concentrations as low as $7 \times 10^{14} \mathrm{~cm}^{-3}$ and mobilities of 42,000 $\mathrm{cm}^{2} \mathrm{~V}^{-1} \mathrm{~s}^{-1}$ at $77 \mathrm{~K}$. This is caused by the addition of a small amount of arsine during the column III cycle, since p-type films with low mobility resulted when the growth was performed without the small arsine supply. This drastic improvement in film quality is theorized to be due to either the suppression of arsenic vacancies near the growing interface or enhanced reaction of As- $\mathrm{H}$ with the surface adsorbed $\mathrm{Ga}$ ethyl complexes. However, since a small amount of arsine is supplied continuously the growth rate dependence on TEG flux observed in FME, as shown in figure 1.3, does not saturate completely at the monolayer level. This is in contrast to ALE using completely separated exposures of $\mathrm{Ga}$ and As compounds where complete saturation has been observed. 


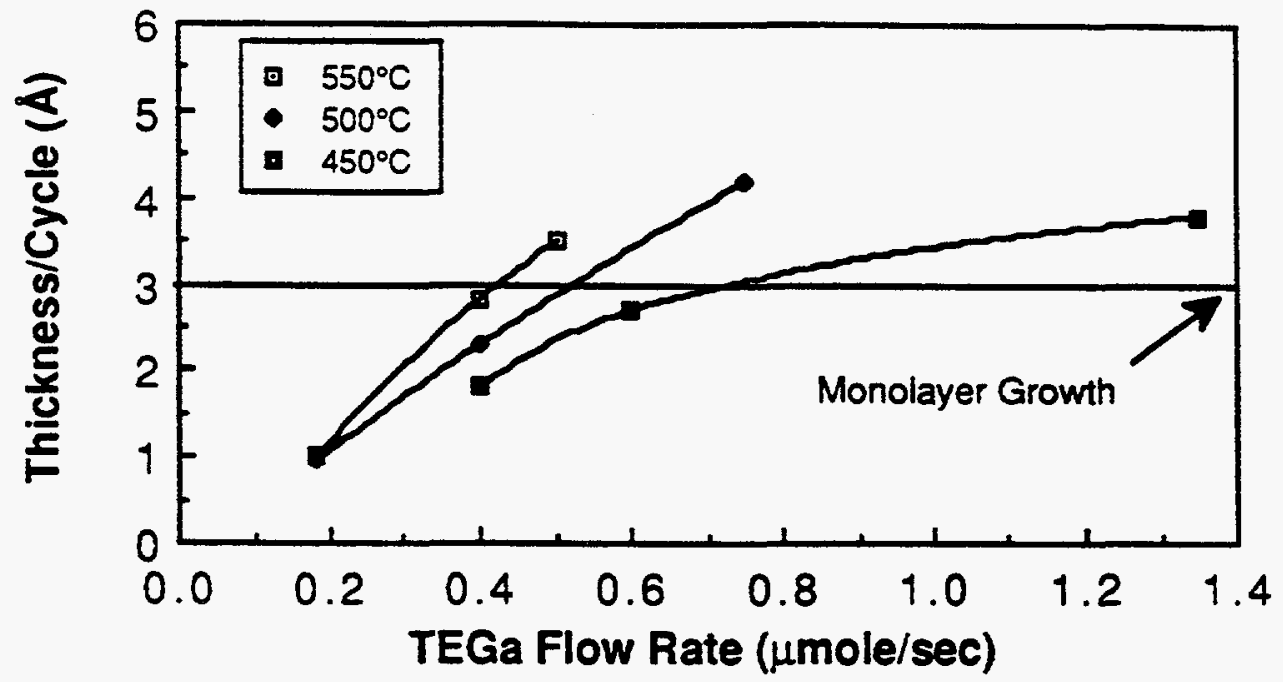

Figure 1.3.

Dependence of growth rate per cycle on TEGa in the FME method.

\subsubsection{Laser-Assisted ALE}

Laser assisted atomic layer epitaxy (LALE) of GaAs was first reported by A. Doi et al. in 1986.20 In this method, modulation of both a $\mathrm{CW}$ Argon ion laser and the gas stream is performed. The laser irradiates the surface during $\mathrm{Ga}$ exposure at an intensity of $100-200 \mathrm{~W} / \mathrm{cm}^{2}$. Utilizing laser irradiation a perfect self-limiting monolayer deposition mechanism was observed over two orders of magnitudes TMGa fluxes. The improved self-limiting nature of LALE in comparison to thermal ALE is theorized to be due to a photocatalytic effect. This is supported by the fact that for laser wavelengths below the band gap of GaAs such as the Nd:YAG laser $(\lambda=1.06 \mu \mathrm{m})$ perfect saturation is not achieved, whereas for two different wavelengths of the Argon ion laser above the band edge 
$(\lambda=514.5 \mu \mathrm{m}, 354 \mu \mathrm{m})$ perfect monolayer saturated growth is observed. A desirable characteristic of selectively deposited GaAs by LALE is the flat-top profile of the deposit in spite of the Gaussian profile of the intensity of the laser beam. This is explained by the fact that the photocatalytic mechanism exhibits a saturation in growth rate as a function of laser power as shown in figure 1.4. This data also supports the theory the observed laser ALE deposition mechanism is not entirely a thermal effect, which would have exhibited a linear growth rate with power density, but rather a surface photocatalytic effect. In addition, the growth rate was found to be independent of temperature in the temperature region of $370-430^{\circ} \mathrm{C}$ which provides further proof that the growth mechanism involves a surface photoreaction.

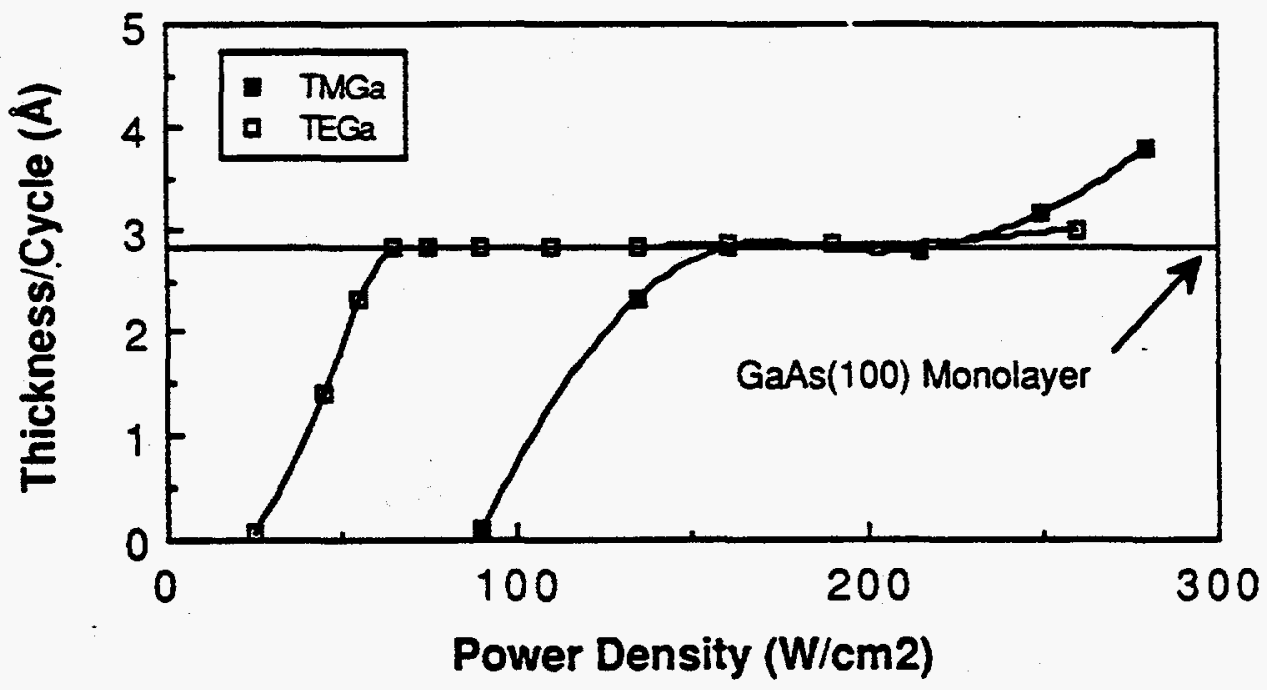

Figure 1.4.

Growth rate as a function of the laser power density in laser-assisted ALE for both TMGa and TEGa. 21

A summary of all the growth rate data for thermal and laser- 
assisted ALE to data is presented in figure 1.5. In comparison to other ALE methods laser-assisted ALE exhibits the best self-limiting growth mechanism. In thermally driven ALE there appears to be a window of conditions over which saturated growth is obtained for various reactor pressures, exposure times, and growth temperatures. For ALE in a high vacuum environment the growth appear to saturate at a rate slightly below a perfect monolayer per cycle because of inefficient cracking of arsine as Nishizawa observed.

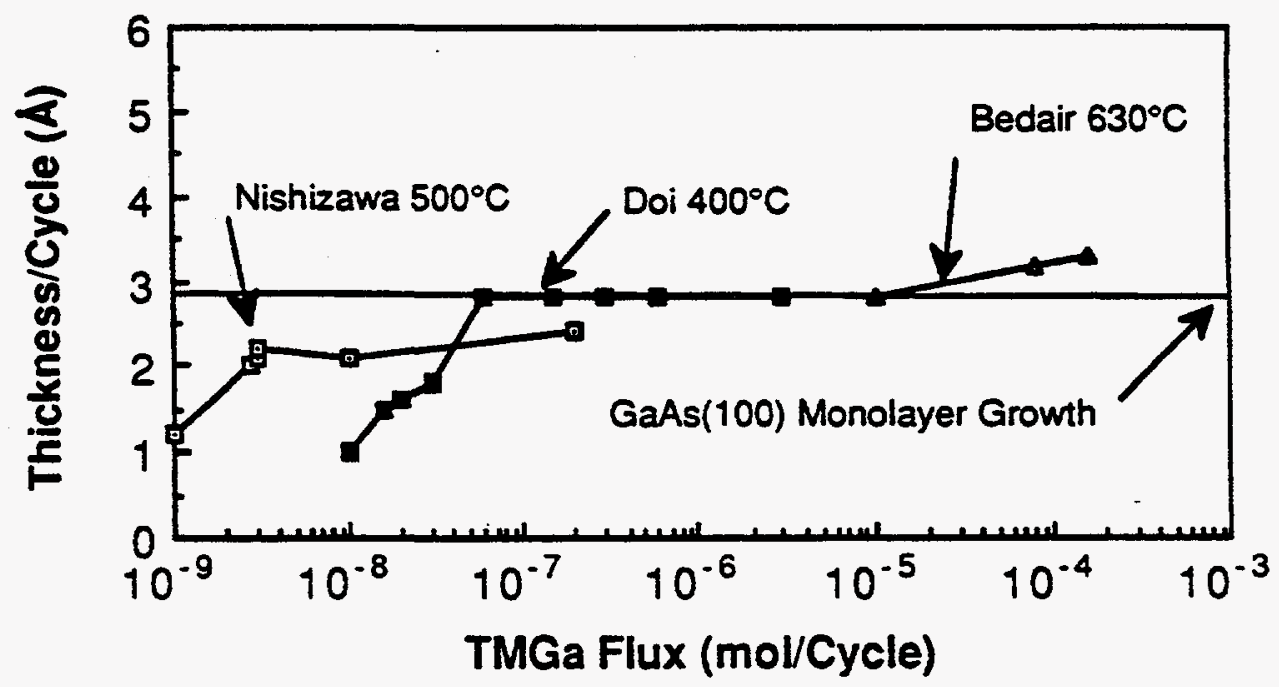

Figure 1.5.

Laser-assisted ALE demonstrates a perfect monolayer per cycle growth rate over a wide range of TMGa fluxes. Thermally driven ALE shows saturated growth over a smaller range of conditions. 


\subsection{Thesis Organization}

This dissertation is organized so that the next two chapters provide the reader with insight into the epitaxial growth and reaction kinetics of conventional MOCVD, because ALE is a variation of this vapor phase epitaxial technique. The fourth chapter describes the experimental conditions, reactor design considerations to achieve ALE, and growth results achieved in thermally driven ALE. Chapter five provides theory and results on ALE grown quantum wells and heterostructure devices. Chapter six consist of photochemical theory and results of laser scanning to achieve laser assisted ALE of GaAs. Chapter seven provides future direction from this research effort on integrated opto-electronic devices and conclusions on the observed experimental results. 
REFERENCES-CHAPTER 1

1 B. K. Gilbert, Gallium Arsenide Technology,( Howard W. Sams \& Co., Inc., Indianapolis, IN, 1985) D. K. Ferry, ed., p.28.

2 J. J. Coleman and P. D. Dapkus, Gallium Arsenide Technology, (Howard W. Sams \& Co., Inc., Indianapolis, IN, 1985) D. K. Ferry, ed., p.91.

3 R. Z. Bachrach, Crystal Growth, 2nd edition(Pergamon Press Ltd., London, 1980) B. R. Pamplin ed., pp. 221-271.

4 H. Kawai, I. Hase, K. Kaneko and N. Watanabe, J. Crystal Growth 68, 508 (1984).

$5 \quad$ P. D. Dapkus, J. Crystal Growth 68, 345 (1984).

6 M. Pessa, P. Huttunen, M. A. Herman, J. Appl. Phys. 54, 6047 (1983).

7 M. Pessa, R. Makela, R. Sunotla, Appl. Phys. Lett. 38, 131 (1980).

8 Y. Aoyagi, A. Doi, S. Iwai, S. Namba, J. Vac. Sci. Technol. B5, 1 460(1987).

9 M. Pessa, R, Makela, R. Suntola, Appl. Phys. Lett.38, 131 (1980)

10 V. P. Tanninen, M. Oikkoinen and T. Tuomi, Thin Solid Films 109, 283 (1983).

11 C. H. L. Goodman and M. V. Pessa, J. Appl. Phys. R65, 60 (1986).

12 M. A. Herman, M. Vulli, and M. Pessa, J. Cryst. Growth 67,339 (1985). 
13 J. Nishizawa, H. Abe, T. Kurabyashi, J. Electrochem. Soc. 132, 1197, (1985).

14 M. Kumagawa, H. Sunami, T. Terasaki, and J. Nishizawa, Japan J. Appl. Phys. 7, 1332 (1968).

15 A. Usi and H. Sunakawa, Inst. Phys. Conf. Ser. No. 79, 753 (1985).

16 M. A. Tischler,and S. M. Bedair, Appl. Phys. Lett. 48, 1961 (1986).

17 M. A. Tischler, N. G. Anderson, and S. M. Bedair, Appl. Phys. Lett. 49, 1199 (1986).

18 N. Kabayashi, T. Makimoto, and Y. Horikoshi, Jap. J. Appl. Phys. 24, L962 (1985).

19 M. Yoshida, H. Watanabe, and F. Uesugi, J. Electrochem. Soc. 130, 413 (1985).

20 A. Doi, Y. Aoyagi, S. Namba, Appl Phys. Lett. 48, 1787, (1986).

21 Y. Aoyagi, A. Doi, S. Iwai, and S. Namba, J. Vac. Sci. Technol. B5(5), 1460 (1987). 


\section{CHAPTER 2}

\section{EPITAXIAL GROWTH BY CONVENTIONAL METALORGANIC CHEMICAL VAPOR DEPOSITION}

\subsection{Introduction}

In the past few years, metalorganic chemical vapor deposition (MOCVD) has evolved into the most promising technique for production of high speed electronic and opto-electronic devices. High purity materials ${ }^{1}$ and monolayer abrupt heterojunctions ${ }^{2}$ have been demonstrated by this technique. Because of the economy and simplicity of the technology, MOCVD is the most viable technique for scale up to large area epitaxial growth for commercial applications. MOCVD is a nonequilibrium vapor phase growth tzchnique which is typically operated at pressures near atmospheric pressure. In this technique a metal alkyl, such as trimethylgallium (TMGa), is mixed in the vapor phase with a hydride, such as arsine $\left(\mathrm{AsH}_{3}\right)$, and the reactants are simultaneously injected into the growth chamber where they thermally decomposed to form single crystal layers.

The MOCVD technique originated from the early research of Manasevit, ${ }^{3}$ who demonstrated demonstrated that triethylgallium (TEGa) and arsine deposited single crystal GaAs pyrolytically in an open tube reactor. Manasevit and co-workers $4,5,6$ subsequently expanded the use of this technique for the growth of $\mathrm{GaAs}_{1-\mathrm{y}} \mathrm{P}_{\mathrm{y}}, \mathrm{GaAs}_{1-\mathrm{y}} \mathrm{Sb}_{\mathrm{y}}$, and $\mathrm{Al}$ containing compounds. Seki et al. ${ }^{7}$ demonstrated that high quality GaAs 
could be obtained by MOCVD by growing films with n-type background doping of $7 \times 10^{13} \mathrm{~cm}^{-3}$ and mobilities of $\mu 77=120,000 \mathrm{~cm}^{2} \mathrm{~V}^{-1} \mathrm{~s}^{-1}$. Bass 8.9 demonstrated the first devices by implementing MOCVD material in field effect transistors (FETs) and photocathode applications. An important milestone in the development of MOCVD was the demonstration of high performance solar cells and double heterostructure lasers by Dupuis and Dapkus. 10,11 This work firmly established that MOCVD could produce high-quality AlGaAs films with excellent optical properties necessary for semiconductor laser applications.

\subsection{System Design}

The overall MOCVD system consists of five major components; source materials, gas handling, reactor chamber, heating system, and exhaust. Only the first three items will be discussed in this section since they are the most pertinent to this work. Figure 2.1 presents a simplified schematic diagram of the components which constitute the MOCVD system employed in this study. 
MFC's (Computer Controlled)

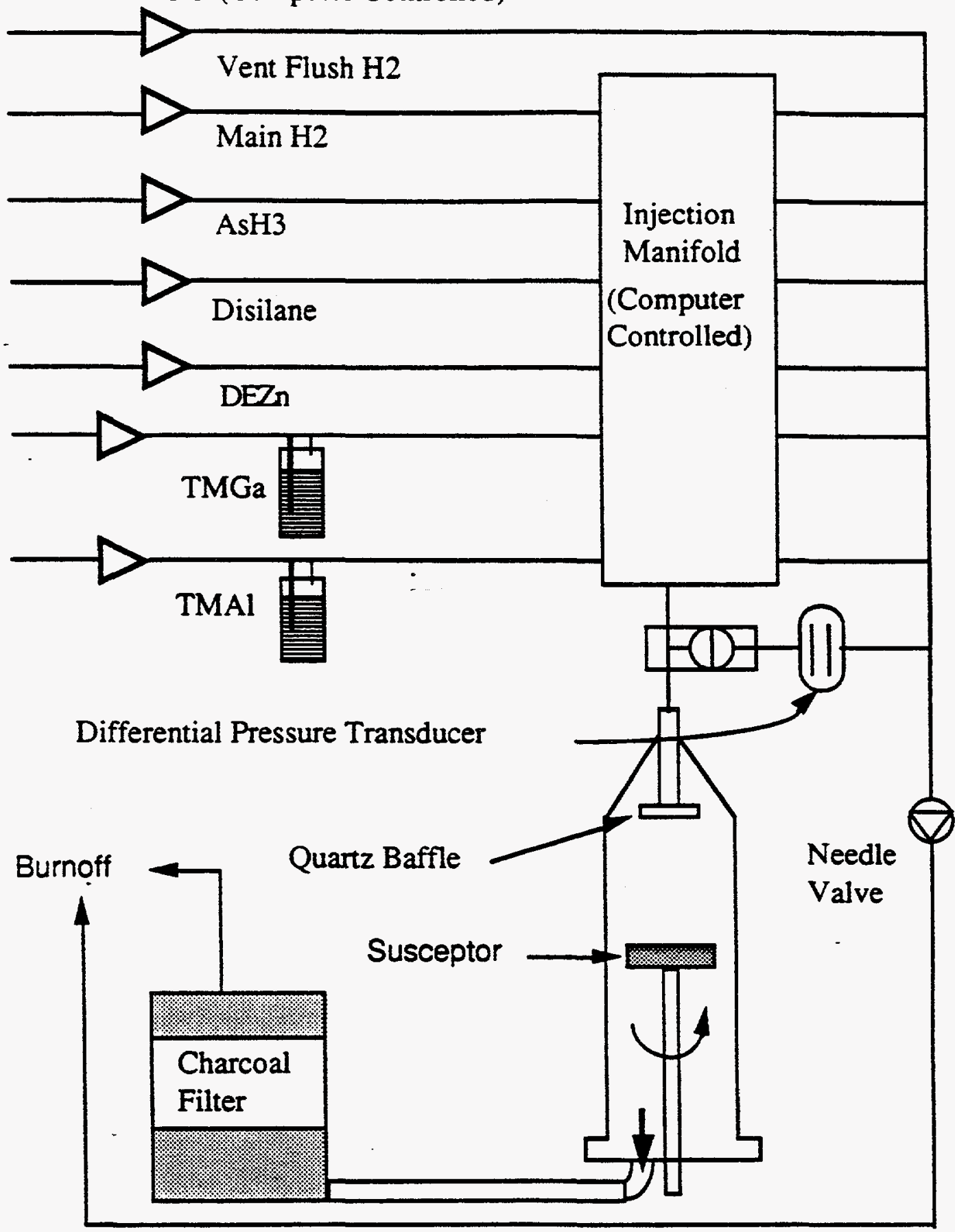

Figure 2.1.

Schematic of conventional MOCVD system employed in this study. 


\subsubsection{Source Material}

Organometallic (OM) sources, such as TMGa and trimethylaluminum (TMAl), provide the group III elements. The OM sources are volatile liquids or solids which are kept in temperature controlled baths to insure a constant equilibrium vapor pressure. The $\mathrm{OM}$ sources are transported to the reactor in the vapor phase by flowing pure $\mathrm{H}_{2}$ through the liquid or over the solid. The amount of the column III precursor transported to the reactor is accurately controlled by precision electronic mass flow controllers to within \pm 0.5 standard cubic centimeter per minute (SCCM). ${ }^{12}$

Group V elements are supplied from gaseous hydride sources such as arsine $\left(\mathrm{AsH}_{3}\right)$ and phosphine $\left(\mathrm{PH}_{3}\right)$. Typically, dilute mixtures of $10 \% \mathrm{AsH}_{3}$ in $\mathrm{H}_{2}$ are contained in high pressure cylinders which incorporate flow-limiting valves for emergency shut-off. On-site purification of the hydride sources is an important concern in the MOCVD process. Both oxygen and water vapor are present in the high pressure gas cylinders at a level of several parts per million (ppm). In this work purification of $\mathrm{AsH}_{3}$ is achieved by both a molecular sieve which adsorbs water, and a $\mathrm{Li}$-impregnated resin. The proprietary Liimpregnated resin (ATM Technology Part \#3010300T) removes both $\mathrm{H}_{2} \mathrm{O}$ and $\mathrm{O}_{2}$ to levels lower than 10 parts per billion (ppb) by an exothermic exchange reaction. 13

High purity hydrogen (99.999\%) is used as the primary carrier gas. A palladium cell hydrogen purifier and an oxygen remover are employed 
to obtain the high level of purity necessary for state-of-the-art GaAs quality.

\subsubsection{Gas Handling}

The gas handling system is designed to deliver accurately measured amounts of pure reactants to the growth chamber. Precision control of the gas flow rates is achieved by electronic mass flow controllers (MFC), manufactured by Tylan Corp. or Unit Instruments, Inc. The design also transports the reactants to the growth chamber without any gas concentration transients due to pressure imbalances or flow changes by incorporating pressure sensors and auxiliary flow balances lines. The gas injection manifold consists of a series of fast gas switching valves employed in a vent/run configuration. An important point to note in figure 2.1 is the addition of pressure transducer between the vent and run lines; this eliminates gas concentration fluctuations resulting from pressure imbalances. Growth is initiated by switching the column III precursors from the vent to run line. An equal flow of hydrogen is switched from the run to the vent line instantaneously to eliminate any pressure surges. Computer controlled (Apple II) switching of the valves enables sharp transitions in the gas composition in the reactor. This allows the MOCVD technique to achieve the monolayer compositional transitions necessary for sophisticated heterostructure devices. 


\subsubsection{Reactor Design}

The most common reactor chamber designs are the vertical and horizontal configurations. In the vertical design reactants are injected through the top. Typically, the substrate is held flat on a rotating silicon carbide coated graphite susceptor which is perpendicular to the gas flow direction. Heating is accomplished by radio frequency (RF) induction, and temperature monitoring is accomplished by either an infrared pyrometer or a thermocouple. The low gas velocities employed in our conventional MOCVD reactor $(2-4 \mathrm{~cm} / \mathrm{sec})$ promote laminar flow conditions. However, convection currents do occur in this design and the flow pattern directly above the wafer is not well understood. The addition of a quartz disc baffle at the gas inlet promotes uniform gas distribution.

The horizontal design utilizes a susceptor which is situated approximately parallel to the gas flow direction. Minimal convection currents and highly laminar gas flow conditions exists in the horizontal reactors. 14 Uniform growth can be achieved only after precise tilting of the susceptor to eliminate reactant depletion along the flow direction. Heating in this design can be accomplished by either RF induction or infrared heating from quartz-halogen lamps.

\subsection{Basic Reaction Chemistry}

The exact chemical pathways operative in the MOCVD process are not yet clearly understood. The basic reaction most commonly used to 
describe the growth of the compound semiconductor GaAs by MOCVD is as follows:

$$
\mathrm{Ga}\left(\mathrm{CH}_{3}\right)_{3(\mathrm{v})}+\mathrm{AsH}_{3}(\mathrm{v})-\cdots \mathrm{GaAs}(\mathrm{s})+3 \mathrm{CH}_{4}(\mathrm{v})
$$

This balanced expression ignores the fact that the specific reaction path and reactive species are largely unknown. A more likely reaction pathway leading to growth of the GaAs epitaxial layers involves the homogeneous decomposition of $\mathrm{TMGa}$ as was reported in an earlier study. 15,16

$$
\mathrm{Ga}\left(\mathrm{CH}_{3}\right)_{3(\mathrm{v})}+3 / 2 \mathrm{H}_{2}(\mathrm{v}) \rightarrow->\mathrm{Ga}(\mathrm{v})+3 \mathrm{CH}_{4}(\mathrm{v})
$$

Arsine is thought to decompose heterogeneously to yield As4 at normal growth temperatures

$$
2 \mathrm{AsH}_{3}(\mathrm{~s} / \mathrm{v}) \rightarrow 1 / 2 \mathrm{As} 4(\mathrm{v})+3 \mathrm{H}_{2}(\mathrm{v})
$$

Therefore the growth of GaAs which probably occurs at the solid vapor interface could be expressed as follows:

$$
\mathrm{Ga}(\mathrm{s} / \mathrm{v})+1 / 4 \mathrm{As} 4(\mathrm{~s} / \mathrm{v})^{--->} \mathrm{GaAs}(\mathrm{s})
$$

However, the level of understanding of the growth process is inadequate from a scientific point of view. The most difficult topic, and certainly the least developed, is the area of the kinetics of the process and growth 
mechanisms occurring at the solid/vapor interface during MOCVD growth. This subject is discussed in detail in Chapter 3.

Most researchers have optimized their MOCVD reactors by empirical studies of external parameters such as growth temperature, V/III ratio, substrate tilt and mass flow rates. However, these studies yield little information about the mechanistic steps which eventually lead to epitaxial growth.

These studies have identified three region of growth: mass transport limited, desorption and surface kinetically limited regimes. As shown in figure 2.1 , kinetics of surface reactions dominate the growth in the low temperature regime. Conventional MOCVD is usually performed in the mass transport limited regime which occurs over a wide temperature range $\left(500^{\circ} \mathrm{C}-800^{\circ} \mathrm{C}\right)$. At high temperatures desorption of arsenic begins to limit the growth and a decrease in the growth rate becomes evident. 


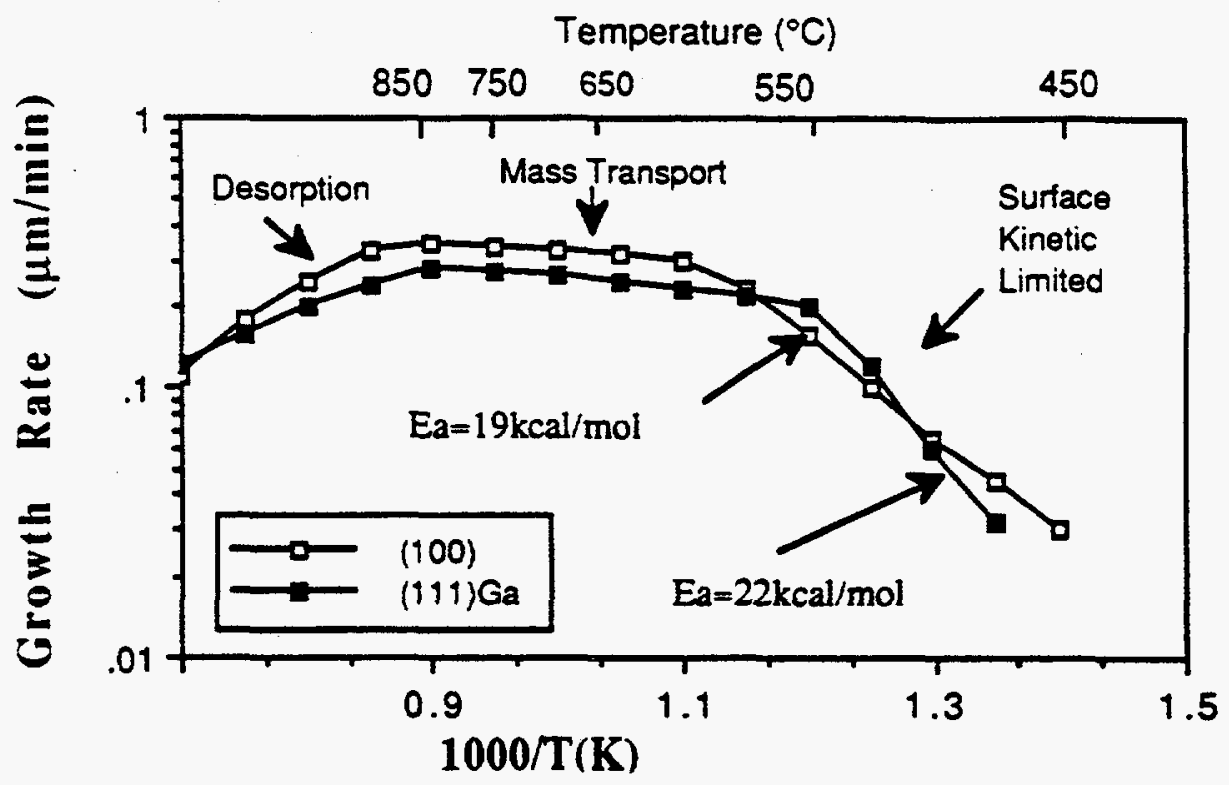

Figure 2.2.

Growth rate versus reciprocal temperature for epitaxial layers on (100) and (111) Ga oriented GaAs substrates, from Reep and Ghandi. 17

\subsubsection{Mass Transport Limited Regime}

In this temperature region, $550-850^{\circ} \mathrm{C}$, growth is limited by mass transport of the column III reactant to the growing interface. Because the diffusion process is slightly temperature dependent, there exists a slight increase in the growth rate in this temperature range as shown in figure 2.2. From Fick's law we can describe the flux of column III elements toward the substrate in the mass transport limited regime as:

$$
J_{G a}=\frac{D_{G a}\left(P_{G a}^{o}-P_{G a}\right)}{\delta_{b} R T}
$$


where $\delta_{b}$ is the boundary layer thickness, $D_{G a}$ the diffusion coefficient for $\mathrm{Ga}$ in the vapor phase, $\mathrm{P}^{\circ} \mathrm{Ga}$ is the input gas stream partial pressure of TMGa, and PGa is partial pressure at the gas-solid interface. For temperatures above $550^{\circ} \mathrm{C}$, the metal alkyl pyrolysis efficiency is unity, then we can assume $\mathrm{PO}_{\mathrm{Ga}} \approx \mathrm{P}_{\mathrm{TMGa}}$. By noting the partial pressure of $\mathrm{Ga}$ at the interface is small in comparison to the input concentration of TMGa ( $\mathrm{P}_{\mathrm{TMGa}}>>\mathrm{PGa}_{\mathrm{Ga}}$ ), we can then express the column III flux at the interface as:

$$
J_{G a}=\frac{D_{G a} P_{T M G a}}{\delta_{b} R T}
$$

From equation 2.7 , we can see that control of the growth rate is easily obtained by controlling the number of moles of metal alkyls injected into the reactor. Precise control of the growth rate is then achieved by utilizing accurate electronic mass-flow controllers which control the flow through the metal alkyl bubblers. As shown in figure 2.3, the growth rate of GaAs in our reactor is directly proportional to the flow rate through the TMGa bubbler . 


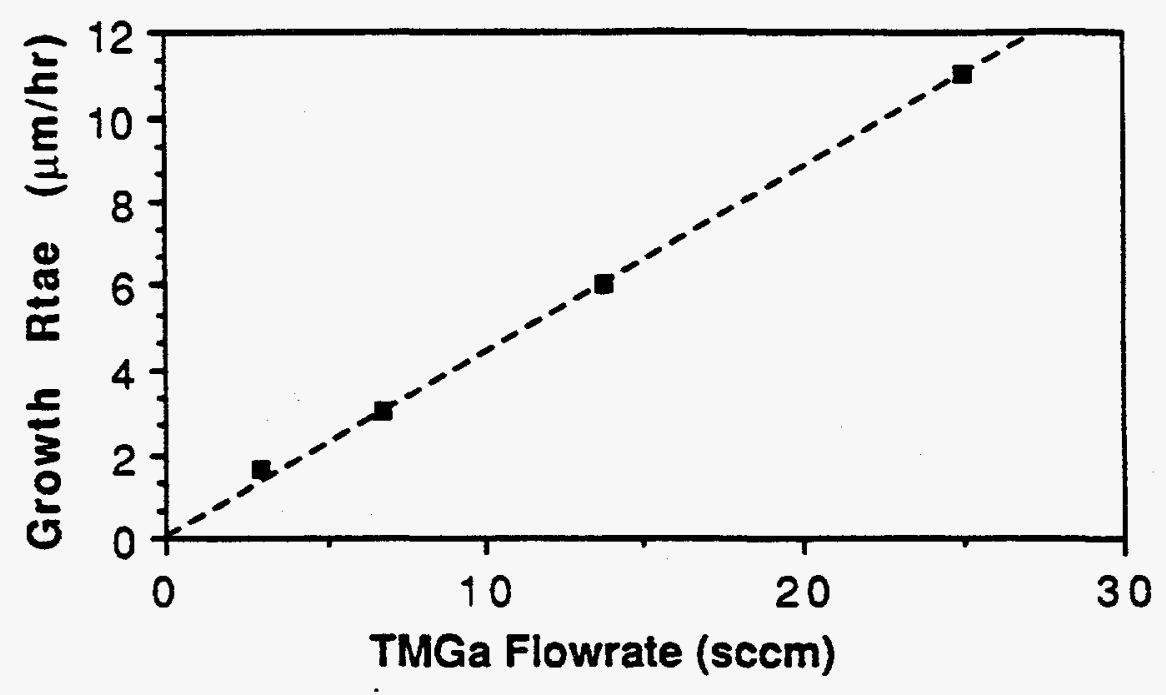

Figure 2.3

Growth rate as a function of TMGa flowrate shows linear dependence in the mass transport limited regime $\left(\mathrm{T}_{\text {growth }}=750^{\circ} \mathrm{C}\right.$ ).

\subsubsection{Surface Kinetic Limited}

In the temperature regime $300-550^{\circ} \mathrm{C}$, the kinetics of surface reactions dominate the growth. Reep and Ghandi17 theorized that the growth process is dominated by adsorption and decomposition of arsine at the growing interface. This view is supported by the fact that the activation energy for the heterogeneous decomposition of arsine on single crystal $\mathrm{GaAs}(100)$ surfaces of $18 \mathrm{kcal} / \mathrm{mol}$ that we have previously reported, 15 is approximately equal to the activation energy of $19 \mathrm{kcal} / \mathrm{mol}$ observed for growth on the $\mathrm{GaAs}(100)$ orientation. However, the presence of TMGa still plays an important role since the growth rate is still strongly influenced by the partial pressure of $\mathrm{TMGa}$ in the reactor. 


\subsubsection{Desorption Limited}

The high temperature region where the growth rate begins to decrease is classified as the desorption limited regime. Several factors may cause the observed drop in the growth rate. One view is that desorption of $\mathrm{Ga}$ from the surface limits the growth rate at these temperatures. Desorption of As2 or As4 may also reduce the growth rate in this high temperature regime. Pre-deposition of the reactants on the reactor sidewalls is yet another mechanism which can limit growth at these temperatures. 18

\subsection{Hydrodynamic Concerns}

\subsubsection{Boundary Layer Effects}

The flow dynamics and temperature gradients occurring near the hot susceptor play an important role in the growth process. Two flow regimes develop in a horizontal reactor. 19 A low-velocity boundary layer develops next to the susceptor. Flow is laminar, and steep thermal and concentration gradients exist in this region. The second is the mixed flow regime in which flow is turbulent and relatively flat concentration and temperature gradients exist. Figure 2.4 of a reactor tube crosssection illustrates the boundary layer and mixed flow zones 


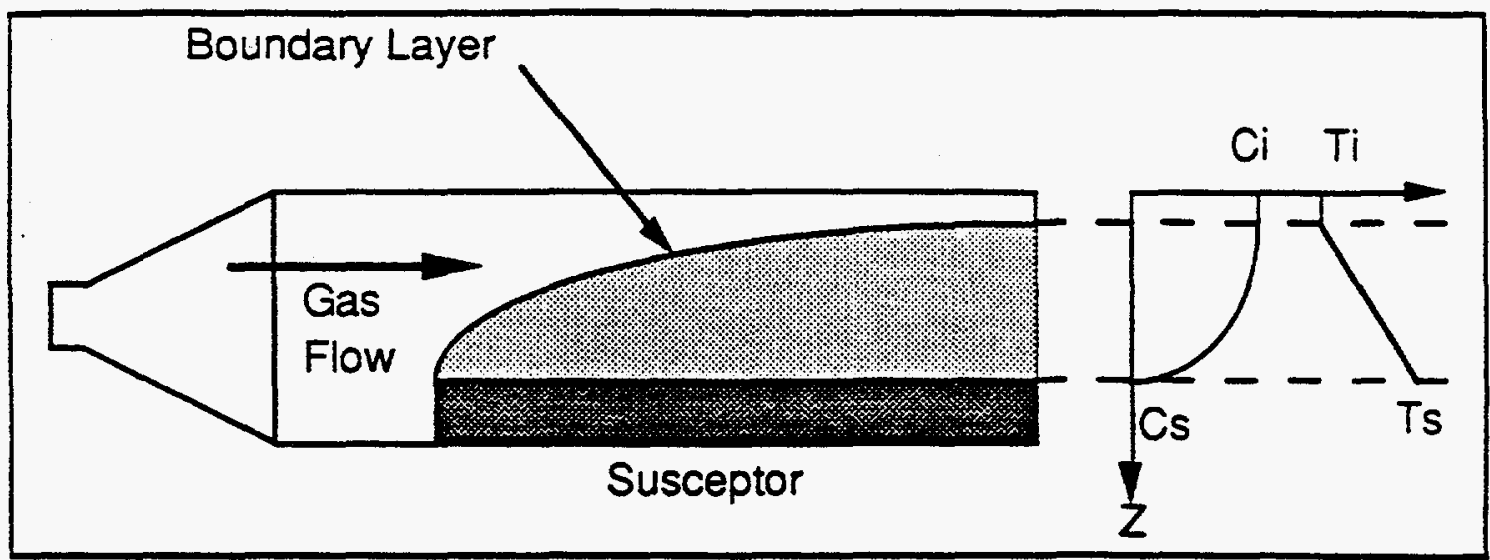

Figure 2.4 .

Schematic illustration of boundary layer in a horizontal MOCVD reactor. Note temperature and concentration profiles in the boundary layer (modified from Berkman et al.).21

Transport of the precursors to the growing interface is controlled by diffusion through the boundary layer zone above the wafer. The reactant concentration decreases along the direction of flow because of consumption ty the growth. Berkman et al.20 have developed a detailed model which accurately predicts mass transport in a horizontal cold wall reactor. For a flat susceptor, their model projects an exponential decrease in the growth rate along the flow direction

Growth in our horizontal MOCVD reactor exhibits the predicted gas phase depletion behavior. Figure 2.5 displays the severe decrease in the layer thickness along the flow direction. Data was taken by measuring the photoluminescence emission energy of the quantum well as a function of wafer position with a tightly focussed laser spot. Well thicknesses were then estimated from a Kronig-Penney model of expected emission energies. This data illustrates the inherent uniformity disadvantage of conventional MOCVD. As we will demonstrate in 
Chapter 5, extremely uniform quantum wells can be grown by ALE because it employs a self-limiting deposition mechanism.

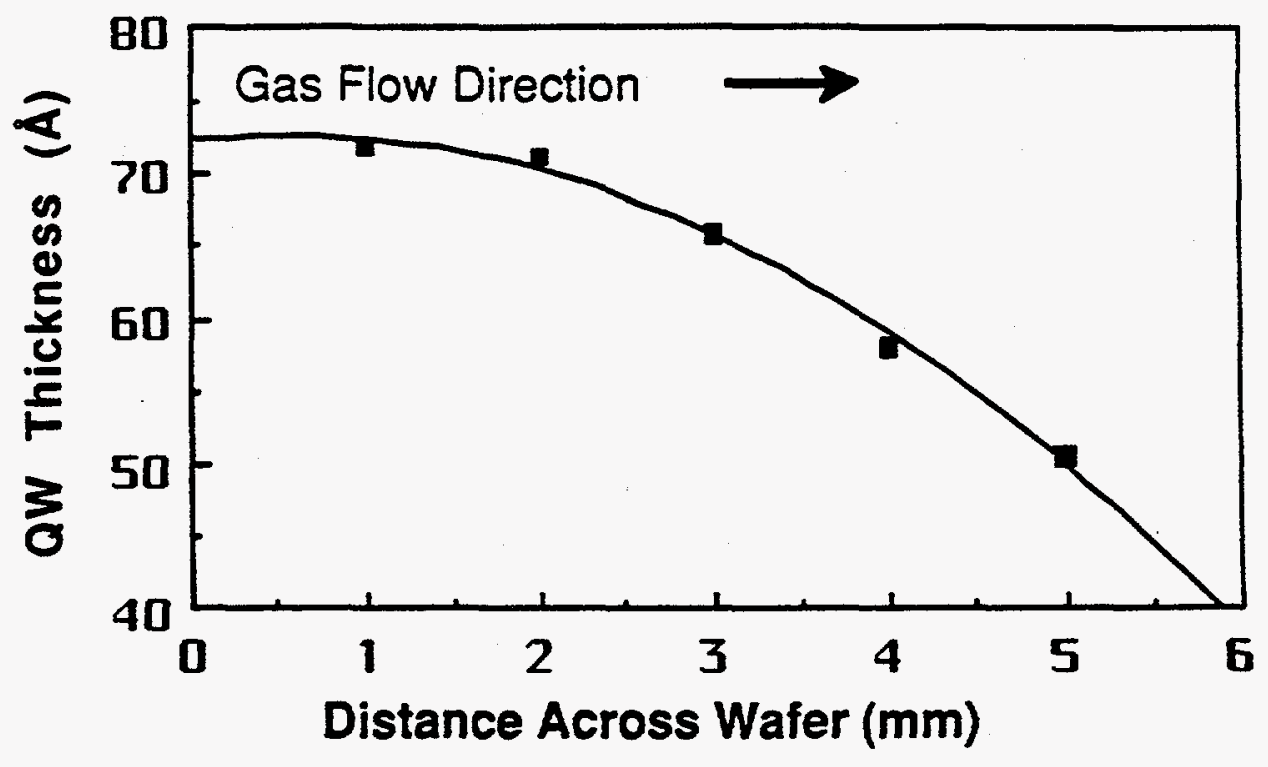

Figure 2.5

QW thickness versus wafer position on sample grown in conventional horizontal MOCVD reactor. Deposition was performed on a flat susceptor to exemplify the gas depletion effect.

By optimizing the hydrodynamic design of the reactor, improvements in the uniformity of the crystal growth can be achieved. Berkman's model predicts that the exponential variation can be minimized by tilting the susceptor at a unique angle $\left(\theta_{c}\right)$.

$$
\sin \left(\theta_{c}\right)=\left(\frac{2 D_{o}}{h_{c} v_{0}}\left[\frac{T_{a}}{T_{0}}\right]^{0.88}\right.
$$

where $D_{0}$ is the diffusion coefficient of the precursor molecule measured at the inlet temperature $T_{0}, T_{a}$ is the average temperature in the boundary 
layer, $h_{c}$ is the channel height above the susceptor, and $V_{o}$ is the initial gas velocity.

Several factors need to be taken into account to achieve uniform growth. As shown in equation 2.8, temperature effects the critical angle needed for uniform growth. Therefore growing layers at different temperatures requires readjustment of several of the process parameters. The total gas-flow rate should be optimized since it affects the complex nature of the boundary layer. Rotation of the susceptor also improves the uniformity of the epitaxial layer. These hydrodynamic concerns become magnified when MOCVD is scaled-up for commercial applications.

\subsubsection{Compositional Control}

For the growth of AlGaAs the solid composition is found to be proportional to the flux ratio of $\mathrm{Al}$ and $\mathrm{Ga}$ at the growing interface. Since the diffusion coefficients of $\mathrm{Al}$ and $\mathrm{Ga}$ are approximately equal, composition control of $\mathrm{Al}_{\mathrm{x}} \mathrm{Ga}_{1-\mathrm{x}} \mathrm{As}$ is achieved by linear adjustment of the molar flows of the column III sources. Data in figure 2.6 illustrates this simple relation of composition in the solid phase to mole fraction in the gas phase. This simple relation is described by the following equation:

$$
X_{A 1}=\frac{M F_{A 1}}{\left(M_{A 1}+M F_{G 2}\right)}
$$

This equation illustrates one of the greatest assets of the MOCVD growth process, that being the distribution coefficient is essentially unity. This 
makes the growth of Al-In compounds possible, which is in contrast to liquid phase epitaxy (LPE) where the growth of Al-In compounds are impossible because of the large liquid distribution coefficient. 20

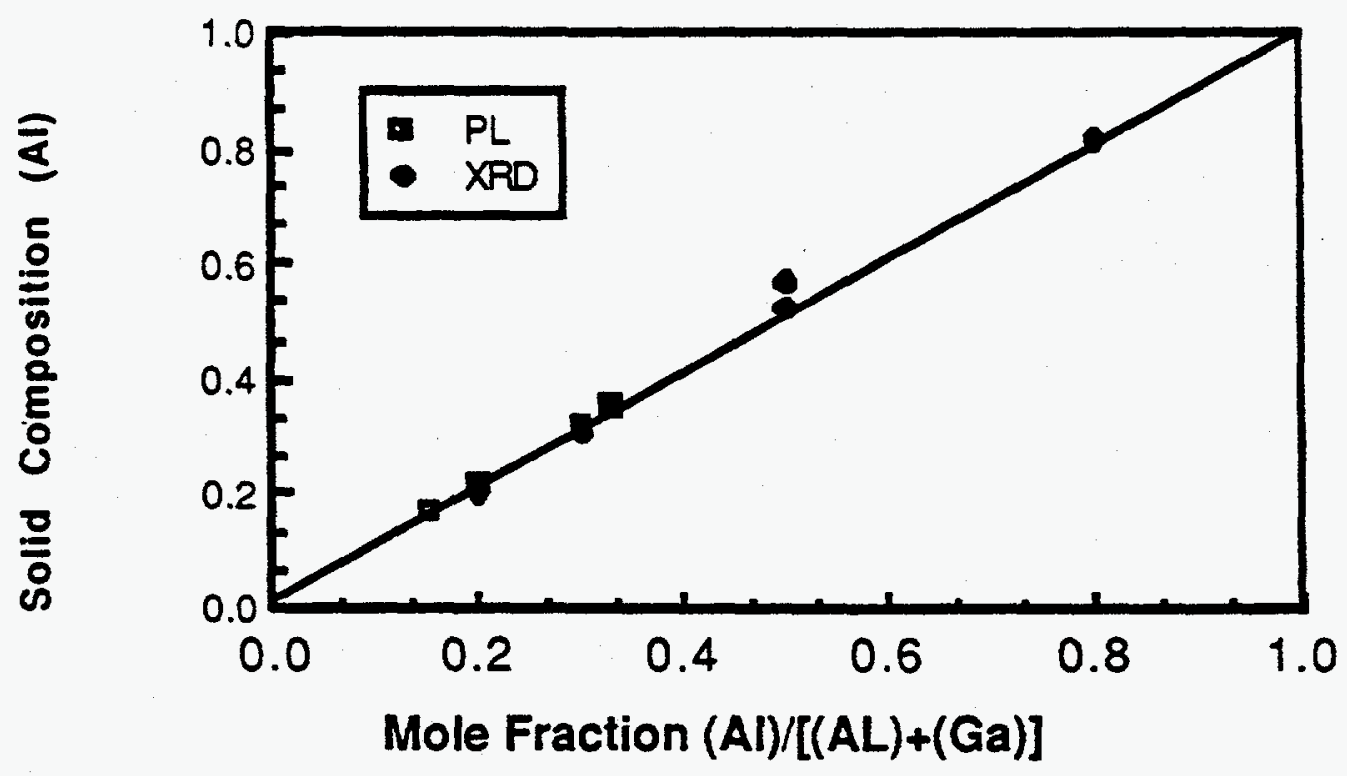

Figure 2.6

Compositional control in MOCVD is achieved by linear adjustment of the molar fraction of the constituents. X-ray Diffraction determined composition data is from H. C. Lee. 21

\subsection{Doping in MOCVD}

\subsection{1 p-Type Doping}

A Group II impurity occupying a group-III sub-lattice position will create a hole in the lattice and therefore cause p-type doping. The standard group-II p-dopant used in MOCVD is $\mathrm{Zn}$ from either DMZn or DEZn precursors. For our MOCVD system, we selected DEZn as the source of p-doping. Dopant incorporation exhibits a temperature activated dependence of electrically active zinc into the lattice. For our 
studies, we observe an activation energy of $1.8 \mathrm{eV}$. The dopant concentration will also depend on the mole fraction of $\mathrm{Zn}$ to $\mathrm{Ga}$ injected into the reactor. DEZn is reported to have a sublinear dependence on the mole fraction. 22 For our reactor, we also observed a sublinear dependence of doping on the mole fraction. 23 Equation 2.10 predicts the doping incorporation of electrically active $\mathrm{Zn}$ in $\mathrm{GaAs}$ for our MOCVD reactor taking into account these factors. This equation is valid for

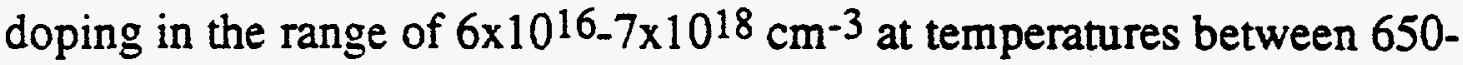
$800^{\circ} \mathrm{C}$.

$$
\mathrm{p}=1.13 \times 10^{29}\left[\exp \left(-\frac{1.8 \mathrm{eV}}{\mathrm{kT}}\right)\right]\left\{\frac{\mathrm{MF}_{\mathrm{Zn}_{\mathrm{n}}}}{\mathrm{MF}_{\mathrm{Ga}}}\right\}^{0.85}
$$

A drawback to using $\mathrm{Zn}$ as a dopant is its high diffusivity at typical growth temperatures. Carbon is an ideal p-type dopant since it has a very low diffusivity value even at high concentration. 24 Recently, Bhat et al. 25 have utilized ALE to grow a carbon doped p-type base in a heterojunction bipolar transistor. They have determined that high carbon doping could be achieved by proper control of the ALE injection sequence and growth temperature.

\subsection{2 n-Type Doping}

Silicon is utilized as the n-type dopant source in our MOCVD system. Si was selected because of its low vapor pressure and low diffusion coefficient to preserve abrupt doping profiles. Disilane was 
selected as the $\mathrm{Si}$ source because of its has an efficient cracking pattern with no temperature dependence. However, for our system we observed a significant dependence of incorporation rate on the temperature. 26 This is believed to be caused by the decomposition of disilane into silane in our source bottle.

\subsection{Conclusions}

This chapter reviews the basic concepts underlying the conventional MOCVD growth process. Although MOCVD processes are not the main topic of this dissertation, the process serves as the base technology upon which we have built our work in ALE. In addition, the shortcomings of the process provide some of the motivation for our work. In particular understanding the surface reaction kinetics of MOCVD precursors, and obtaining uniform growth motivate the need for studying ALE. 
REFERENCES-CHAPTER 2

1 S. K. Shastry, S. Zemon, D. G. Kenneson, G. Lambert, Appl. Phys. Lett. 52, 150 (1988).

2 S. J. Jeng, C. M. Wayman, G. Costrini, and J. J. Coleman, Materials Lettters 2, 359 (1984).

3 H. M. Manasevit, Appl. Phys. Lett. 12, 156 (1968).

4 - H. M. Manasevit and W. I. Simpson, J. Electrochem. Soc. 116, 1725 (1969).

5 . H. M. Manasevit, J. Electrochem. Soc. 118, 647 (1971).

6 H. M. Manasevit and K. L. Hess, J. Electrochem. Soc. 126, 2031 (1979).

7 Y. Seki, K. Tanno, K. Lida, and E. Ichiki, J. Electrochem. Soc. 122, 1108 (1975).

8 S. J. Bass, J. Crystal Growth 31, 172 (1975).

9 S. J. Bass, J. Crystal Growth 44, 29 (1978).

10 R. D. Dupuis, P. D. Dapkus, R. D. Yingling, and L. A. Moudy, Appl. Phys. Lett., 31, 201 (1977).

11 R. D. Dupuis and P. D. Dapkus, Appl. Phys. Lett., 32, 472 (1978).

12 Unit Instuments, Inc. Orange, California, Sales Brochure (1986).

13 Advanced Technology Materials, Stamford, Conn.,Technical Sheet (1987).

14 L.J. Giling, J. de Physique, C5, 235 (1982). 
15 S. P. DenBaars, B. Y. Maa, P. D. Dapkus, A. D. Danner, H. C. Lee, J. Crystal Growth, 77, 188, (1986).

16 J. Nishizawa, H. Abe and T. Kurabayashi, J. Electrochem. Soc. 132, 1197 (1985).

17 D. H. Reep and S. K. Ghandi, J. Electrochem. Soc. 130, 675 (1983).

18 W. Richter, "Physics of Metal-Organic Chemical Vapour Deposition," Festkorperprobleme XXVI ( Pergamon Press, New York, 1986).

19 S. Berkman, V. S. Ban, and N. Goldsmith, Heterojunction Semiconductors for Electronic Devices, G. W. Cullen and C. C. Wang, eds., (Springer-Verlag, Berlin. 1979) p. 264.

20 J. S. Yuan, C. C. Hsu, R. M. Cohen, and G. B. Stringfellow, J. Appl. Phys., 57, 1380 (1985).

21 H. C. Lee, PhD. Dissertation, University of Southern Califomia, (1987) p.106.

22 V. Aebi, C. B. Cooper, R. L. Moon, and R. R. Saxena, J. Crystal Growth, 55, 517 (1981).

23 A. D. Danner, $\mathrm{PhD}$. Dissertation, University of Southern California (1987) p.74.

24 N. Kobayashi, T. Makimoto, and Y. Horikoshi, Appl. Phys. Lett, 50, 1435 (1987).

25 R. Bhat, J. R. Hayes, E. Colas, and R. Esagui, IEEE Electron Device Lett., 9, 442 (1988).

26 A. D. Danner, PhD. Dissertation, University of Southern California (1987) p.72. 


\section{CHAPTER 3 \\ REACTION KINETICS STUDY OF \\ METALORGANIC CHEMICAL VAPOR DEPOSITION}

\subsection{Introduction}

To achieve atomic level control of the MOCVD process a study of the reaction kinetics involved in the thermal decomposition of metalorganic precursors would be invaluable. Currently, the understanding of the kinetics and mechanisms of gas phase and surface reactions operative in the MOCVD process is limited. Thermodynamics predicts that every closed isolated system will approach an equilibrium state, in which its properties are independent of time. The second law of thermodynamics can estimate the amount of driving force for epitaxy. For the equation typically used to describe the MOCVD process, thermodynamics tell us that the reaction is very exothermic and spontaneous even at room temperature.

$\mathrm{Ga}\left(\mathrm{CH}_{3}\right)_{3}+\mathrm{AsH}_{3}->\mathrm{GaAs}+3 \mathrm{CH}_{4} \quad \Delta \mathrm{Ho}_{\mathrm{f}}=-83 \mathrm{kcal} / \mathrm{mole}$

However, thermodynamics is incapable of telling us anything about the chemical pathways or time required to obtain equilibrium. These problems can be solved by applying chemical kinetics, which predict the 
time varying properties of chemical systems. Kinetics are not fast enough to establish equilibrium in the MOCVD process, therefore MOCVD is classified as a non-equilibrium growth technique.

In order to help elucidate critical reaction pathways in MOCVD we have applied an "ex-situ" sampled gas Infrared Spectroscopy (IR) setup to study the thermochemical kinetics. Through the use of this analysis we have been able to effectively measure decomposition reaction rates of gaseous precursors utilized in MOCVD. The homogeneous rates of decomposition are measured whenever possible. In some instances, surface catalytic reactions are dominant and the effect of the surface chemistry on the decomposition is described. These studies allow us to determine separately the homogeneous and heterogeneous reaction rates and to determine the catalytic reactivity of various surfaces and gaseous species. Rate constants measured for these decomposition processes can than be extrapolated to the case of ALE growth in chapter four to explain the observed growth behavior.

\subsection{Reaction Kinetics}

\subsubsection{MOCVD Kinetic Steps}

The growth rate in MOCVD is determined by the slowest process in a series of reactions leading to deposition. Figure 3.1 illustrates some of the plausible reaction pathways which lead to the epitaxial growth of GaAs. Gas phase reactants must diffuse through the boundary layer where several reaction pathways are possible. Pyrolysis of TMGa in the gas phase may provide the source of $\mathrm{Ga}$ atoms needed for crystal growth. 
Alternatively, the formation of an adduct between $\mathrm{TMGa}$ and $\mathrm{AsH}_{3}$ may be the mechanism for $\mathrm{GaAs}$ growth. Another reaction mechanism might occur is the surface reaction of adsorbed gallium monomethyl $\left(\mathrm{GaCH}_{3}\right)$ with arsenic hydride (AsH) to release methane $\left(\mathrm{CH}_{4}\right)$.

$$
\mathrm{GaCH}_{3(\mathrm{ads})}+\mathrm{AsH}_{(\mathrm{s} / \mathrm{v})}-->\mathrm{GaAs}_{(\mathrm{s})}+\mathrm{CH}_{4(\mathrm{v})}
$$

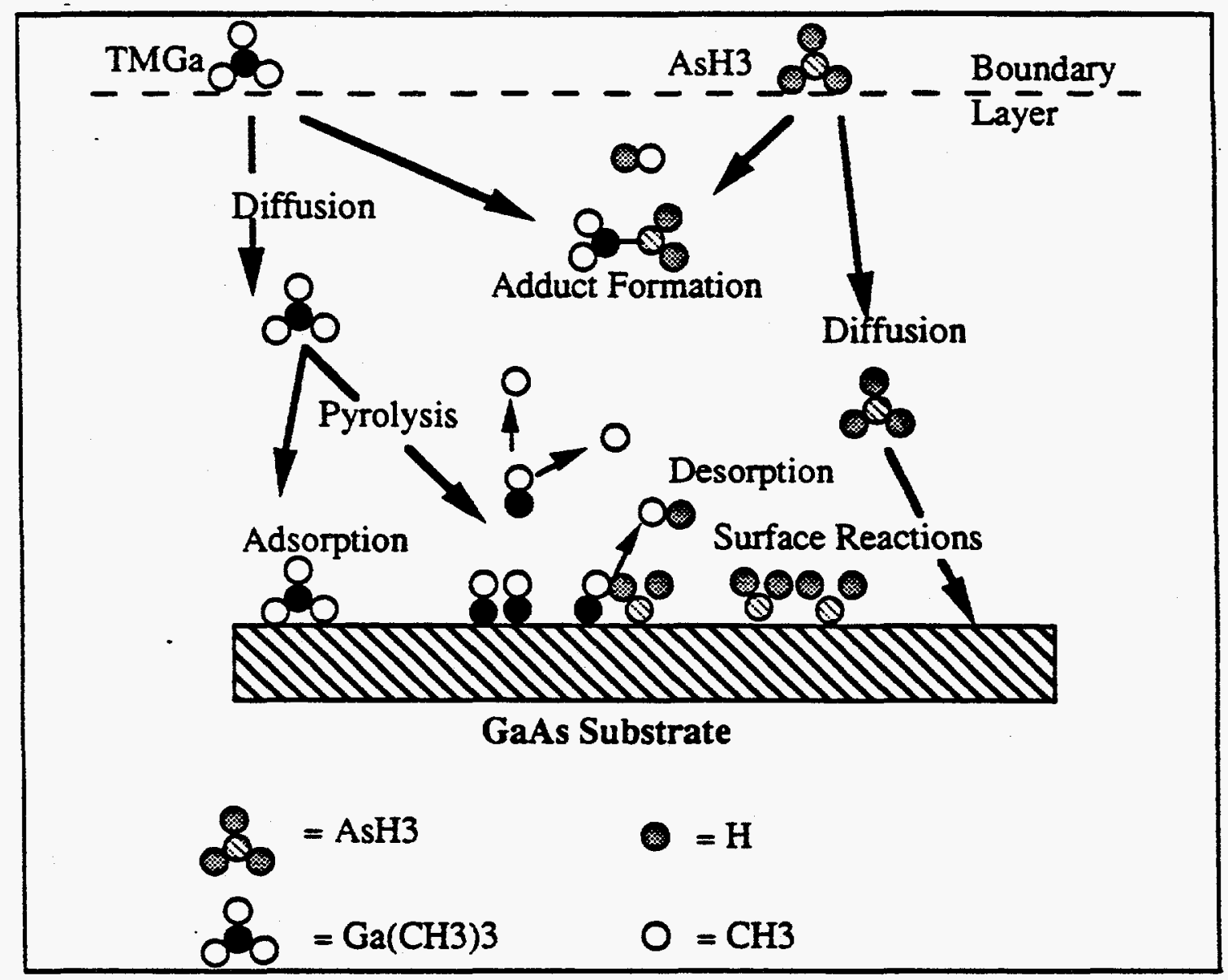

Figure 3.1

Schematic diagram illustrating some of the possible kinetic steps occurring in an MOCVD reactor. 


\subsubsection{Theory of Thermochemical Kinetics}

The most useful quantitative model for describing reaction kinetics is the "activated complex theory" (ACT), also known as the "transitionstate theory (TST) of rate processes." 1 In order for a unimolecular reaction to occur, the reacting molecule must obtain sufficient internal energy to break the necessary bond. For a gas phase reaction this "activation" energy may be accumulated by a sequence of "atypical" collisions with average molecules in the gas. The reaction-coordinate energy diagram shown in figure 3.2 is useful for illustrating the kinetic process. The solid curve is the potential energy of the molecule corresponding to zero kinetic energy for that reaction coordinate. The light horizontal lines suggest the different vibrational levels of the molecule. The energy of the average molecule will be $E^{\circ}{ }_{A}$, while $E^{*} A$ is the energy of the average reacting molecule. Note that at any finite temperature; the average reacting molecule is not precisely at the top of the energy barrier but significantly above it. The activation energy for the reaction of $A-->B$ is given as $E^{*}{ }_{1}$ in the figure. 


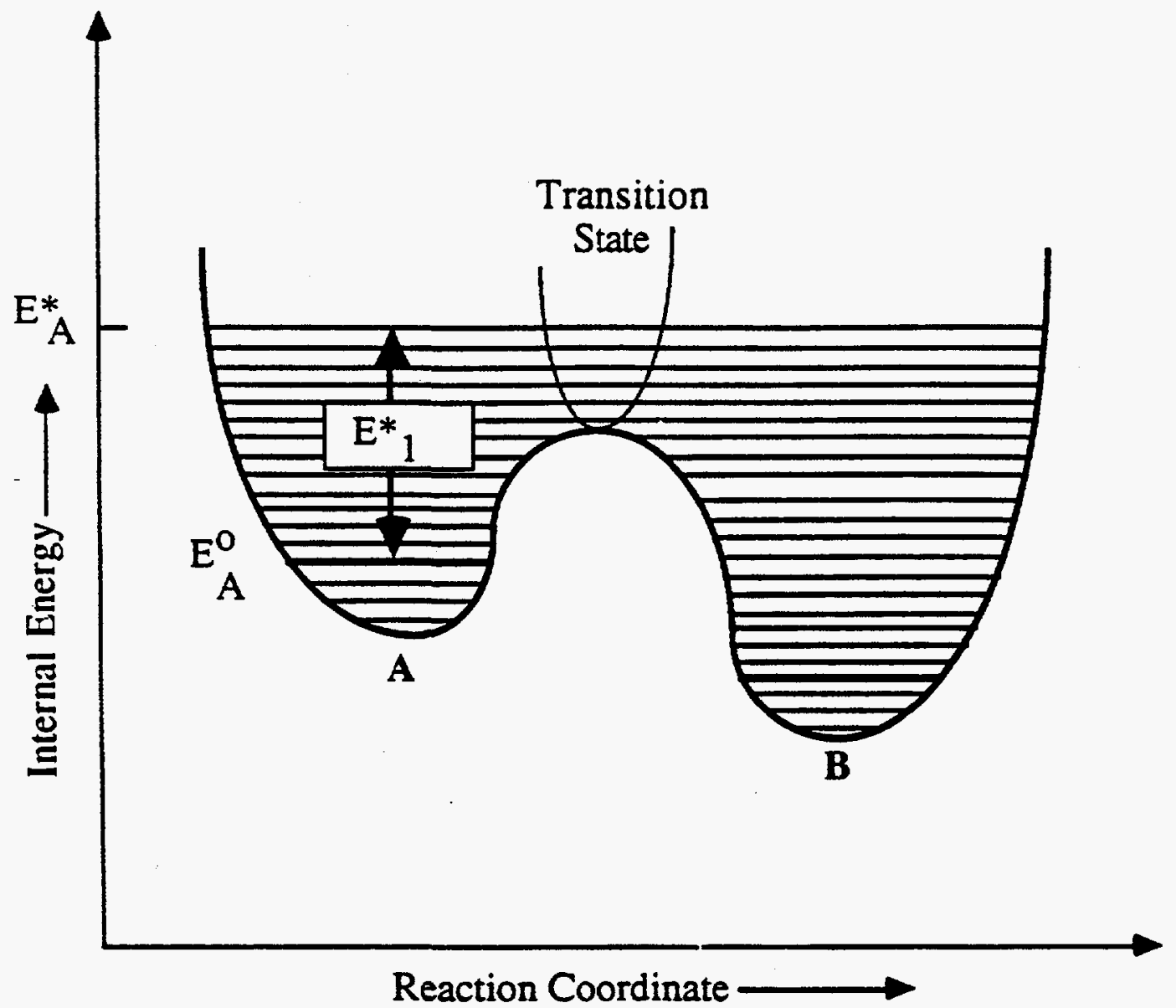

Figure 3.2 Energy-reaction coordinate diagram for simple fission of molecule A->B (after Benson). ${ }^{2}$

To illustrate the fission of a complex metalorganic molecule such as $\mathrm{Ga}\left(\mathrm{CH}_{3}\right)_{3}$, lets first consider the simple, unimolecular fission of a molecule AB.

$$
\mathrm{AB} \leftrightarrow \mathrm{AB}^{*} \leftrightarrow \mathrm{A}+\mathrm{B}
$$

Where $A B^{*}$ is a molecule that has obtain enough internal energy to be in the transition state and can therefore decompose into A and B. The first 
order rate constant for the decomposition of $\mathrm{AB}, \mathrm{k}_{\mathrm{AB}}$, can be related to the activation energy of the process by the Arrhenius equation:

$$
k_{A B}=A * \exp \left(-\frac{E_{a}}{R T}\right)
$$

Where $E_{a}$ is the activation energy, $R$ is the ideal gas constant, $A$ is the frequency factor, and $\mathrm{T}$ is the reaction temperature.

We now introduce thermodynamic formalism to demonstrate the relation between the activation energy and the dissociative bond strength. From S.W. Benson, ${ }^{3}$ the rate constant can also be expressed as:

$$
\mathrm{k}_{\mathrm{AB}}=\left(\frac{\mathrm{kT}}{\mathrm{h}}\right) \mathrm{K}_{\mathrm{AB}}^{*}
$$

where $\mathrm{K}_{\mathrm{AB}}{ }^{*}$ is the equilibrium rate constant of the transition state species for motion across the barrier, $h$ is Planck's constant, and $k$ is Boltzmann's constant. The free-energy change for the transition state reactant is related to the rate constant by:

$$
\Delta \mathrm{G}^{*}=-\mathrm{RT} \ln \left(\mathrm{K}_{\mathrm{AB}}{ }^{*}\right)
$$

By definition :

$$
\Delta \mathrm{G}^{*}=\Delta \mathrm{H}^{*}-\mathrm{T} \Delta \mathrm{S}^{*}
$$

We can then express the rate constant for the decomposition of $A B$ as: 


$$
\mathrm{k}_{\mathrm{AB}}=\left[\frac{\mathrm{kT}}{\mathrm{h}}\right] \exp \left(\frac{\Delta \mathrm{S}_{\mathrm{AB}}^{*}}{\mathrm{R}}\right) \exp \left(-\frac{\Delta \mathrm{H}_{\mathrm{AB}}^{*}}{\mathrm{RT}}\right)
$$

Comparing equation 3.4 to the thermodynamic formalism of the rate constant, equation 3.8 , we can see that the dissociative bond enthalpy is related to the activation energy by:

$$
\mathrm{E}_{\mathrm{a}}=\Delta \mathrm{H}_{\mathrm{AB}}{ }^{*}+R \mathrm{~T}_{\mathrm{m}}
$$

where $T_{m}$ is the mean temperature of experiment. For our experiments the $\mathrm{RT}_{\mathrm{m}}$ contribution is typically small $(1-2 \mathrm{kcal} / \mathrm{mol})$. We can also identify the relation between the frequency factor " $A$ ", and the entropy of dissociation as follows:

$$
A=\left(\frac{e_{k T}}{h}\right) \exp \left(\frac{\Delta S_{A B}^{*}}{R}\right)
$$

Several of the metalorganic precursors, such as TMGa, are believed to decompose by unimolecular gas phase reactions. ${ }^{4}$ Therefore, if we can measure the reaction rates and activation energy for such processes we can measure the dissociative bond strengths for homogeneous gas phase reactions such as the fission of methyl groups from TMGa:

$$
\begin{array}{ll}
\mathrm{Ga}\left(\mathrm{CH}_{3}\right)_{3}->\mathrm{Ga}\left(\mathrm{CH}_{3}\right)_{2}+\mathrm{CH}_{3} & \text { kTMGa } \\
\mathrm{Ga}\left(\mathrm{CH}_{3}\right)_{2-->} \mathrm{Ga}\left(\mathrm{CH}_{3}\right)+\mathrm{CH}_{3} & \text { kDMGa } \\
\mathrm{Ga}\left(\mathrm{CH}_{3}\right)-->\mathrm{Ga}+\mathrm{CH}_{3} & k_{M M G a}
\end{array}
$$




\subsection{Experimental Setup}

The decomposition of $\mathrm{AsH}_{3}$ and TMGa was measured by flowing a mixture of $\mathrm{H}_{2}$ and the desired reactant through a heated quartz tube 5.0 $\mathrm{cm}$ in diameter inserted in a furnace with confined and well controlled hot zone $12.5 \mathrm{~cm}$ long. Figure 3.3 illustrates the experimental setup used in this study of MOCVD reaction kinetics. The concentration of reactants at various temperatures was measured by sampling a portion of the gas at the exit end of the hot zone and using infrared absorption spectroscopy to measure the absorption coefficient for known vibrational transitions of the molecules under study. In some cases, reactive surfaces such as GaAs wafers were inserted into the reactor to determine their effect on the decomposition of the reactants. Eight 2 inch GaAs (100) wafers were installed in sich a way as to insure that most of the gas stream would interact with before exiting the hot zone, see figure 3.4. The absolute concentration of reactants was determined from the known vapor pressure of TMGa, the $\mathrm{H}_{2}$ flows, and from the manufacture's analysis of $\mathrm{AsH}_{3}$ concentration. The evolution of $\mathrm{CH}_{4}$ from the decomposition of TMGa was measured and calibrated relative to a commercial standard. 


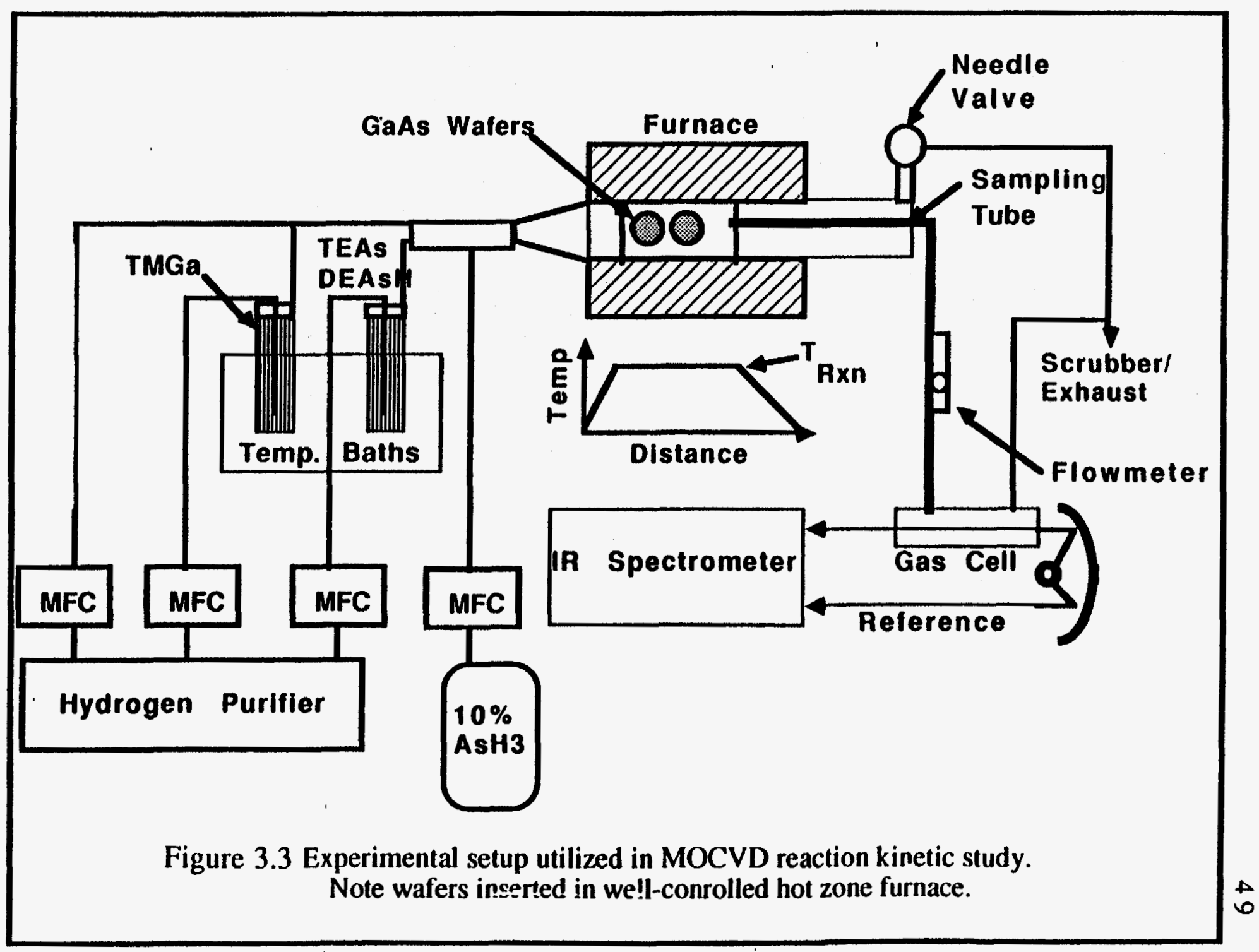




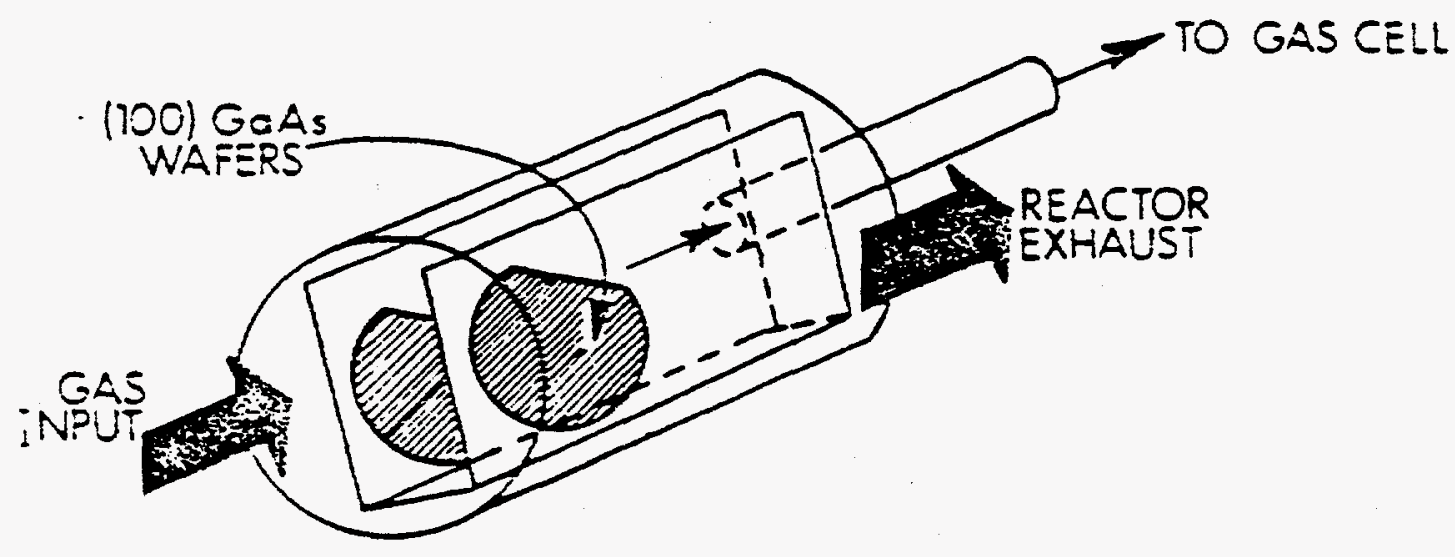

Figure 3.4

GaAs wafers are arranged in reactangular quartz boat to insure maximum maximum interaction with sampled gas stream. 


\subsection{Sampled Gas IR Spectroscopy $\mathrm{TMGa} / \mathrm{AsH}_{3} / \mathrm{H}_{2}$}

\subsubsection{IR Spectra TMGa/ $\mathrm{AsH}_{3} / \mathrm{H}_{2}$}

The infrared absorption spectra of $\mathrm{TMGa} / \mathrm{H}_{2}$ mixtures sampled from a flowing gas stream have been examined in detail at a variety of temperatures. The gas mixture was passed through the hot zone at an average gas velocity of $1.25 \mathrm{~cm} / \mathrm{sec}$. Figure 3.5 shows the room temperature spectra of TMGa which exhibits peaks at $\sim 580, \sim 700, \sim 1200$, and $\sim 2900 \mathrm{~cm}^{-1}$. We have monitored the features at 1200 and $580 \mathrm{~cm}^{-1}$ that are associated with the $\mathrm{C}-\mathrm{H}$ vibrational mode and the $\mathrm{Ga}-\mathrm{C}$ stretching mode respectively, 5 to determine the change in TMGa concentration. Above $400^{\circ} \mathrm{C}$ the spectra show additional features at 1304 and $3014 \mathrm{~cm}^{-1}$ that are associated with $\mathrm{CH}_{4}$ vibrational modes. 


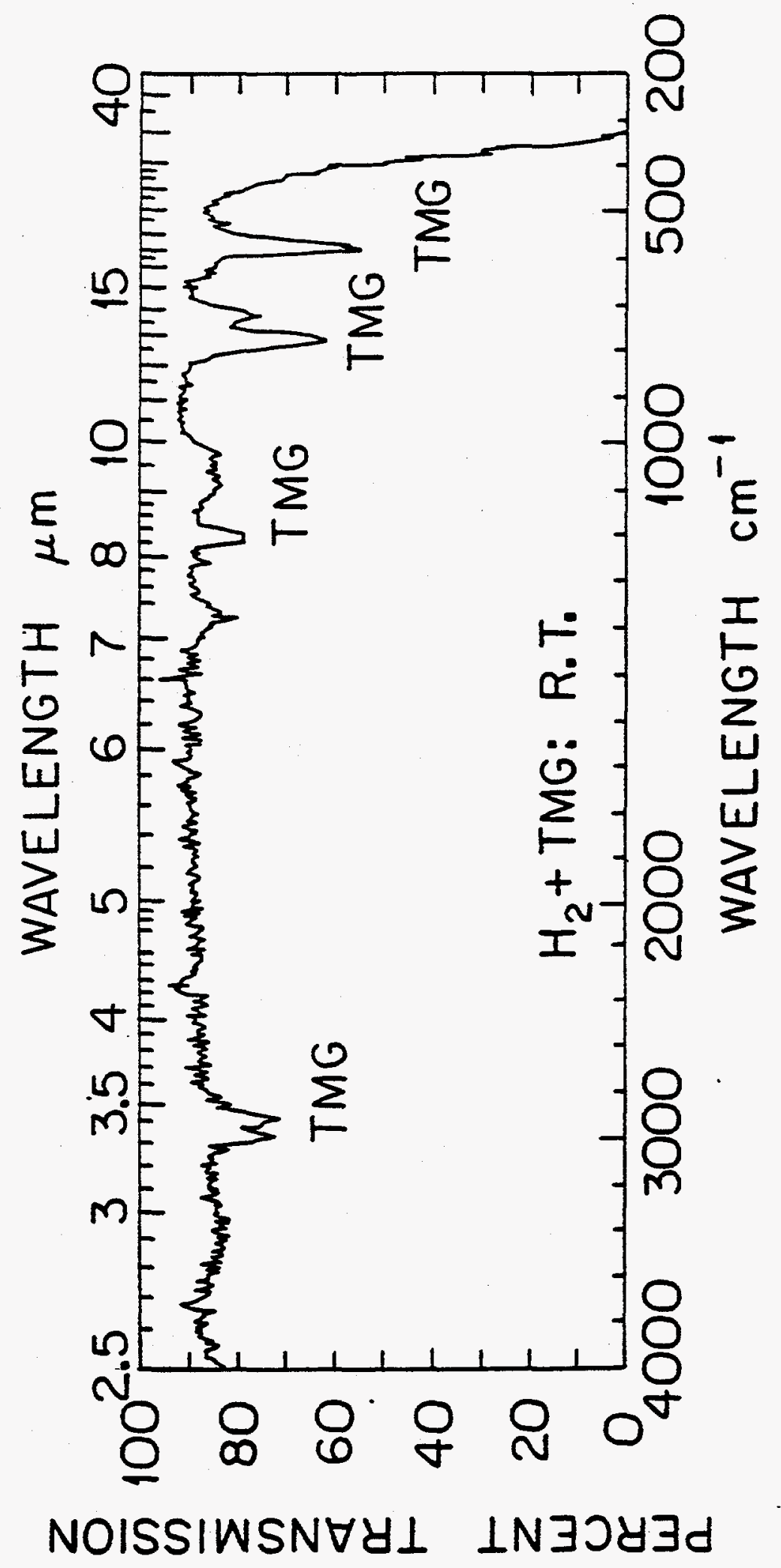


The relative concentration of reactants is obtained from the transmission spectra by the use of Beer's law:

$$
T=T_{0} e^{(-a b c)}
$$

where: $T_{0}$ is the baseline transmission, $a$ is the absorptivity of the molecule at a specified wavelength $\left(\right.$ moles $\left.^{-1} \mathrm{~cm}^{-1}\right), b$ is the path length of the cell $(10 \mathrm{~cm})$, and $\mathrm{c}$ is the molar concentration of the reactant. By measuring the input and output IR transmission spectra, we can determine the final reactant concentration relative to the initial concentration:

$$
\frac{C_{f}}{C_{i}}=\frac{\ln \left(\frac{T_{o}}{T_{f}}\right)}{\ln \left(\frac{T_{o}}{T_{i}}\right)}
$$

Figure 3.6 shows the dependence of the TMGa concentration upon the temperature of the hot zone. Note that the relative concentration is unchanged up to a temperature of $380^{\circ} \mathrm{C}$ and decreases above $400^{\circ} \mathrm{C}$ to zero at $\sim 460^{\circ} \mathrm{C}$. The decomposition curves for $\mathrm{TMGa}$ in quartz tube with and without GaAs wafers in the hot zone show only a small difference. 


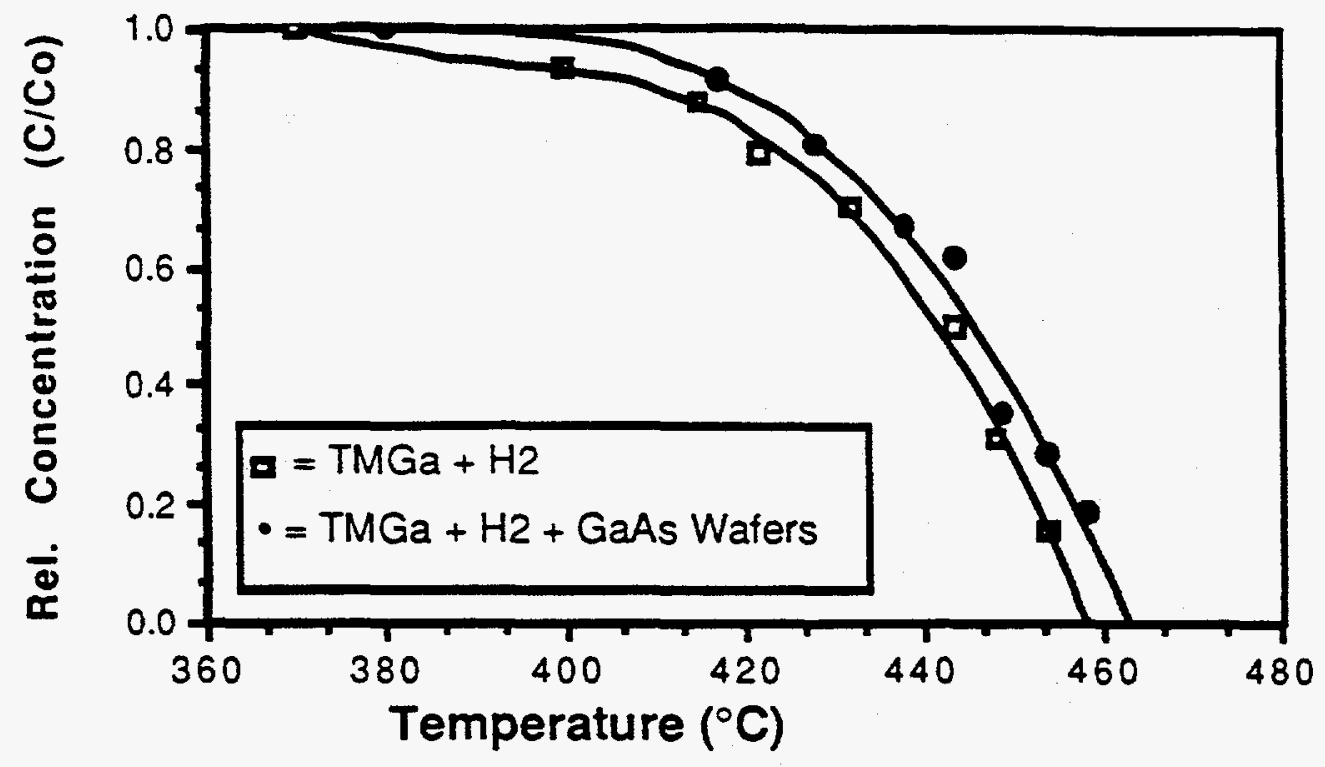

Figure 3.6

Relative concentration of $\mathrm{TMGa}$ in $\mathrm{H}_{2}$ at various temperatures.

We have also measure the evolution of $\mathrm{CH}_{4}$ from the decomposition of TMGa. Figure 3.7 shows the temperature dependence of this evolution for several different gas mixtures. In all cases the formation of $\mathrm{CH}_{4}$ coincides with the onset of TMGa decomposition. The evolution of $\mathrm{CH}_{4}$ continues up to $\sim 500^{\circ} \mathrm{C}$ at which temperature it saturates. We have calibrated the concentration relative to a commercially prepared mixture and find that in all cases the $\mathrm{CH}_{4}$ concentration saturates at $\mathrm{CH}_{4}=3$ TMGa. 


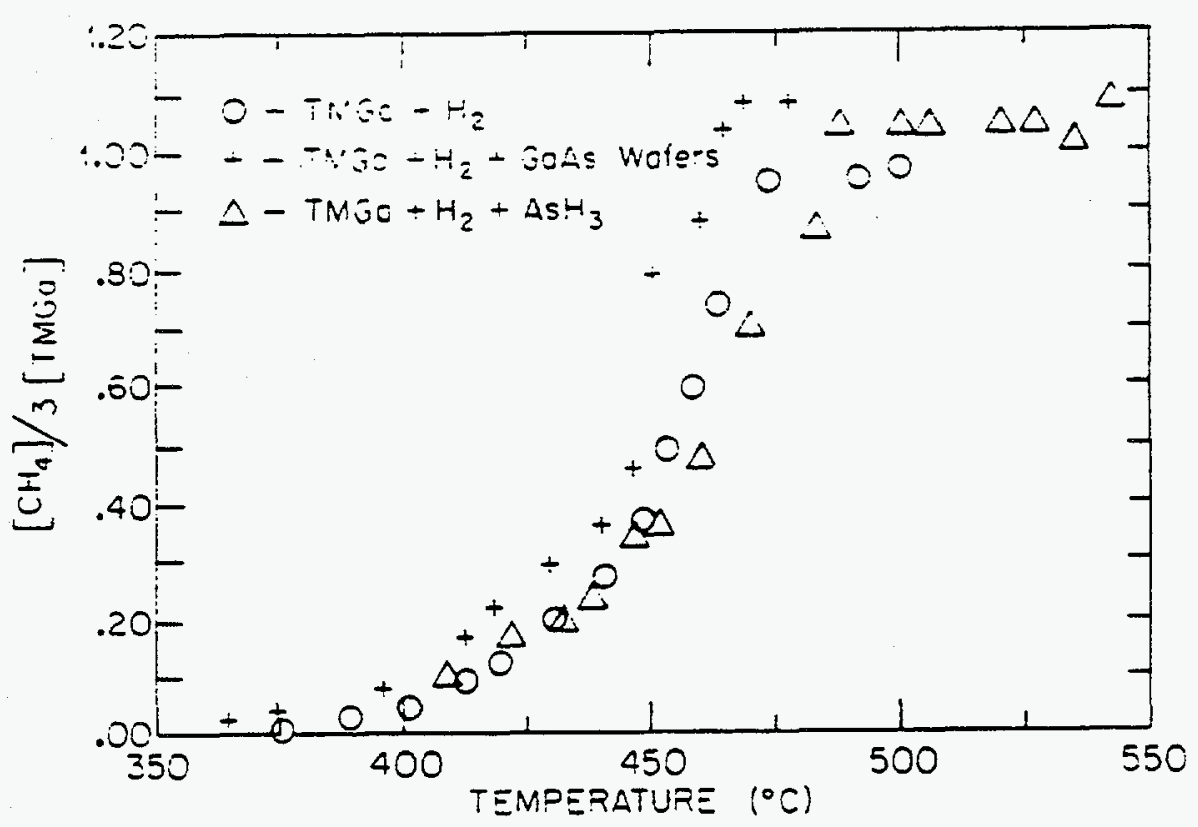

Figure 3.7

Evolution of methane from pyrolysis of TMGa. 
Infrared spectra of $\mathrm{AsH} / \mathrm{H}_{2}$ and $\mathrm{AsH}_{3} / \mathrm{TMGa} / \mathrm{H}_{2}$ mixtures have also been examined at various gas temperatures. Spectral features are observed at $880-1050$ and $1950-2300 \mathrm{~cm}^{-1}$ that are related to $\mathrm{AsH}_{3}$ vibrational modes. The appearance of peak at $2080 \mathrm{~cm}^{-1}$ is observed upon the addition of $\mathrm{TMGa}$ to the $\mathrm{AsH}_{3} / \mathrm{H}_{2}$ system. Nishizawa et al. 6 have also observed the appearance of this unknown peak. We believe this to indicate the formation of a organoarsenic compound in the gas phase. This is based upon examination of the IR spectrum for diethylarsenic hydride (DEAsH) which exhibits a red shifted As- $\mathrm{H}$ vibrational peak also at $2080 \mathrm{~cm}^{-1}$, see figure 3.11 presented later in this chapter. 


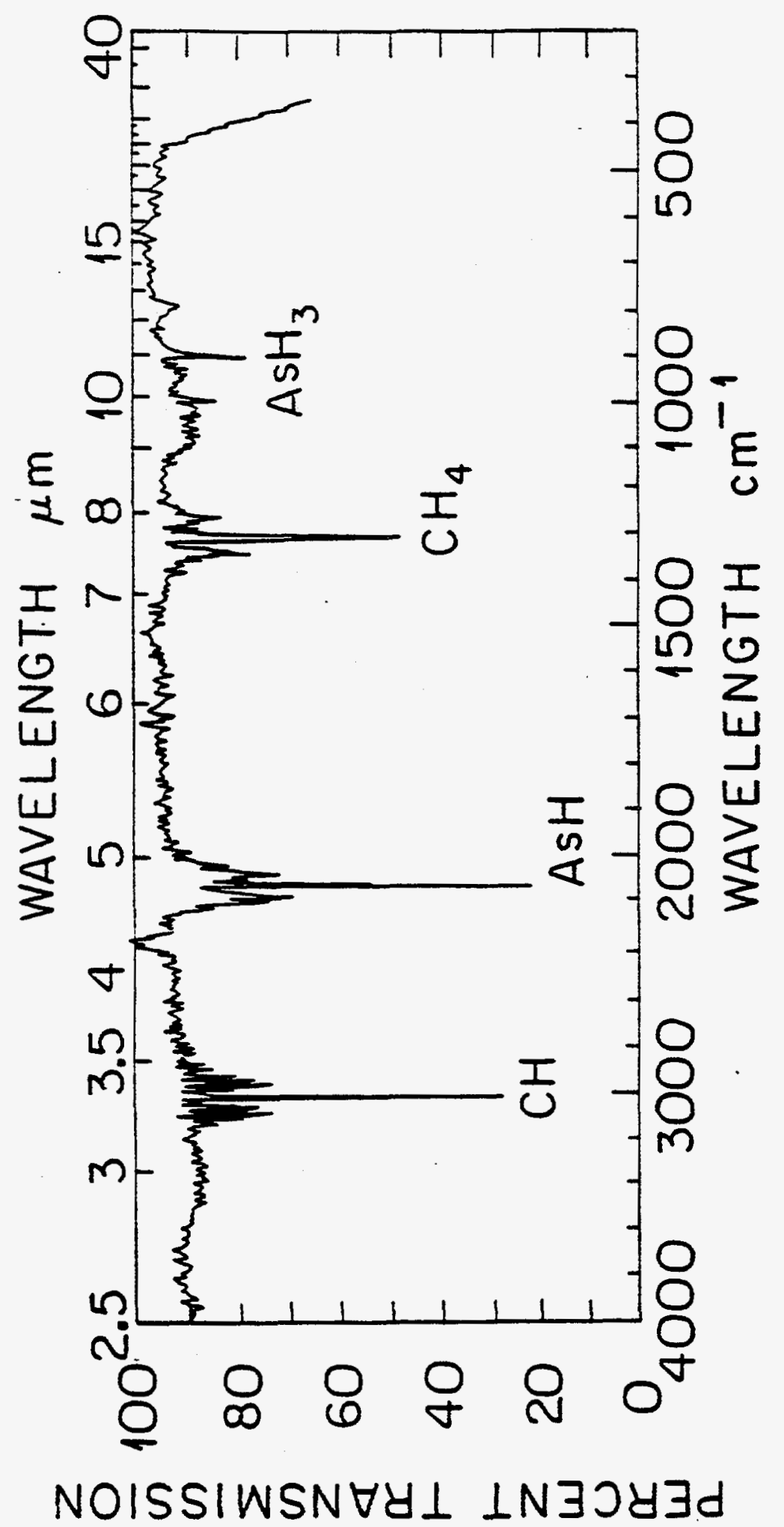


We monitor the feature at $2112 \mathrm{~cm}^{-1}$ to determine the concentration of $\mathrm{AsH}_{3}$ in heated mixtures. Figure 3.9 shows the variation with temperature of the $\mathrm{AsH}_{3}$ concentration measured in this way. Note the gradual decrease in $\mathrm{AsH}_{3}$ concentration with increasing temperature The concentration of $\mathrm{AsH}_{3}$ in $\mathrm{H}_{2}$ is strongly affected by the nature of the surfaces in the reactor, as is indicated by the difference between the decomposition curve obtained in a clean quartz reactor and that obtained when GaAs wafers are present in the reaction zone. The rate of decomposition of $\mathrm{AsH}_{3}$ was also enhanced by the presence of $\mathrm{Ga}$ wall deposits and nonstoichiometric Ga:As wall deposits. The presence of TMGa in the gas phase also increases the rate of $\mathrm{AsH}_{3}$ decomposition, the rate increasing with the TMGa concentration as shown in figure 3.9 In this case it is not possible to separately determine the role of gas phase reactions from those occurring on the reactor walls due to Ga:As deposits that inevitably occur. 


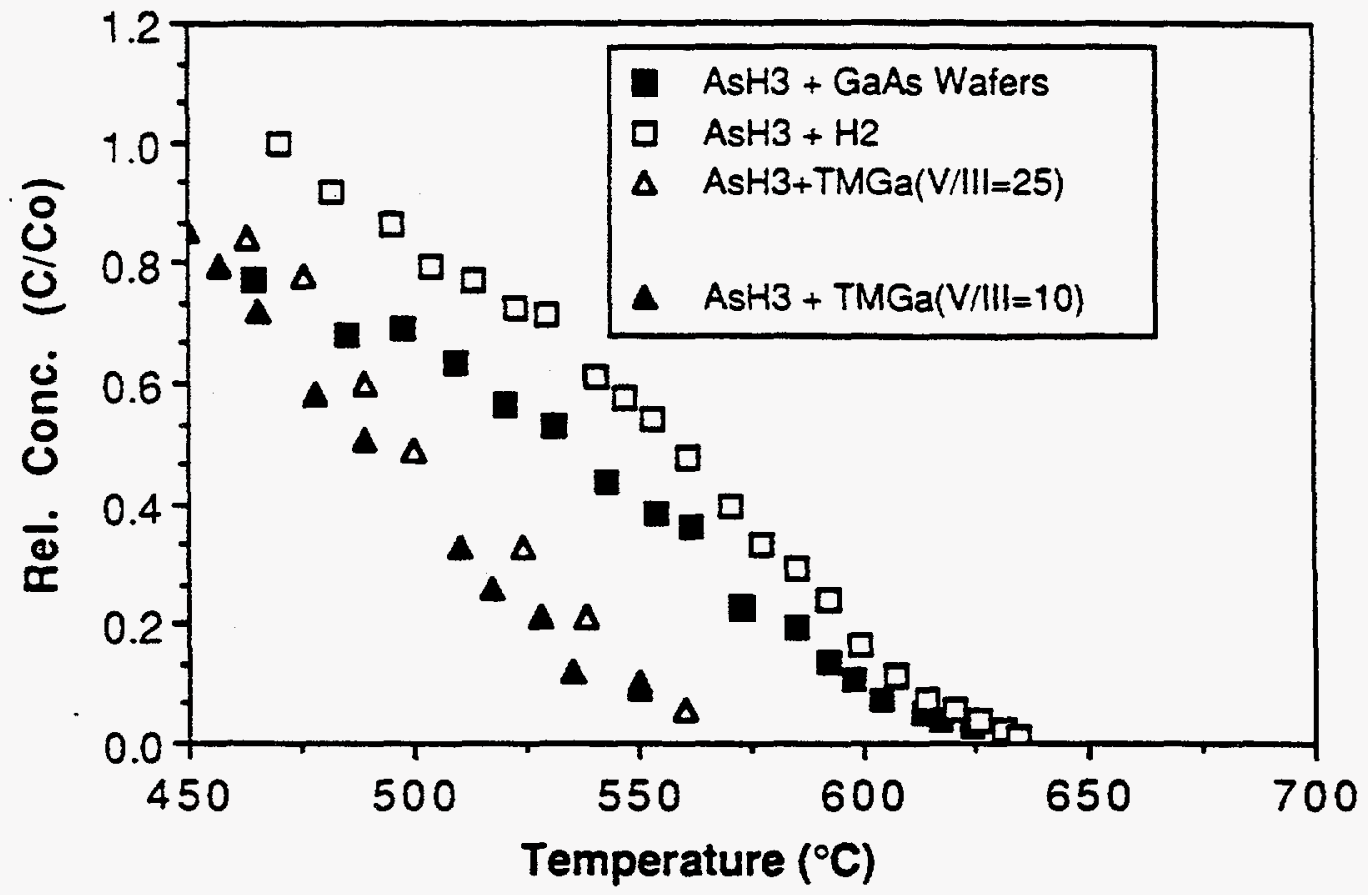

Figure 3.9

Relative concentration of $\mathrm{AsH}_{3}$ in $\mathrm{H}_{2}$ at various temperatures and for several different reactor conditions. Note the strong effect of the surfaces on $\mathrm{AsH}_{3}$ decomposition.

\subsubsection{Activation Energies}

The data of figures 3.6-9 can provide further insight into the reaction processes occurring in an MOCVD reactor. Yoshida et al. ${ }^{4}$ have shown that the decomposition of TMGa is a first order reaction. The kinetics for this decomposition process can be described by:

$$
\frac{\mathrm{dC}}{\mathrm{dt}}=-\mathrm{kC}
$$

where $C$ is the concentration of the reactant and $\mathrm{k}$ is a rate constant of the Arrhenius form: 


$$
k=A \exp \left(-\frac{E_{a}}{R T}\right)
$$

A has the units of $\sec ^{-1}$ and $E_{a}$ is the activation energy for the process. As derived in section 3.2.2, for a homogeneous unimolecular process $E_{a}$ will reflect the dissociative bond strength of the Ga-C bond in TMGa. We determine $\mathrm{k}$ by integrating equation 3.16 :

$$
\int_{C_{0}}^{C_{f}}\left(\frac{d C}{C}\right)=-\int_{0}^{\Delta t} k d t
$$

where $\Delta t$ is the contact time in the reactor hot zone. This leads to the following expression for the effective reaction rate constant:

$$
k=\frac{\ln \left(\frac{C_{o}}{C_{f}}\right)}{\Delta t}
$$

From equation 3.17 we can deduce that a logarithmic plot of $k$ versus $1 / T$ will yield the activation energy for the process under study.

$$
\mathrm{E}_{\mathrm{a}}=\frac{\partial \ln (\mathrm{k})}{\partial\left(\frac{1}{\mathrm{RT}}\right)}
$$

Figure 3.10 shows a plot of $\ln \mathrm{k}$ versus $1 / \mathrm{T}$ for the decomposition of TMGa in hydrogen. The slope of the straight line fit to the data of 
figure 3.8 yields an activation energy of $E_{\mathrm{a}}=58 \mathrm{kcal} / \mathrm{mol}$ for this process and $E_{O}=62 \mathrm{kcal} / \mathrm{mol}$ for decomposition in the presence of GaAs wafers. It is not known whether the difference in activation energy observed in these two cases is significant or merely a measure of experimental error. Both activation energies are comparable to the activation energy for the loss of one methyl group from TMGa that was measured by Jacko and Price ${ }^{7}(59.5 \mathrm{kcal} / \mathrm{mol})$ which is an approximate measure of the dissociative bond enthalpy for the first methyl group on $\left(\mathrm{CH}_{3}\right)_{3} \mathrm{Ga}$, i.e. $\mathrm{DH}\left(\left(\mathrm{CH}_{3}\right)_{2} \mathrm{Ga}-\mathrm{CH}_{3}\right)$.

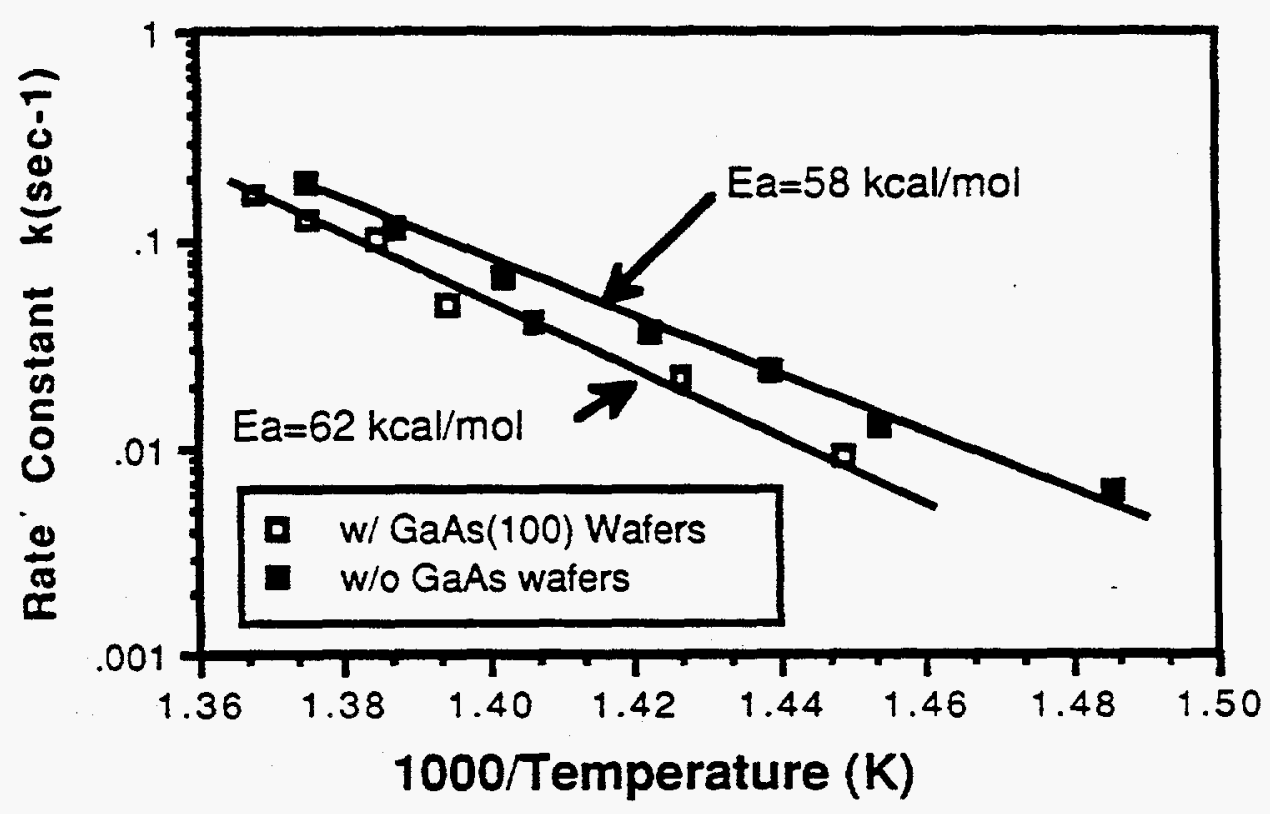

Figure 3.10

Effective decomposition rate constant versus reciprocal temperature for the decomposition of TMGa.

Jacko and Price observed no more than two $\mathrm{CH}_{4}$ molecules for each TMGa molecule decomposed at any temperature in their studies with a 
tolulene carrier. The data of figure. $3.7 \mathrm{can}$ be further analyzed to yield information related to the decomposition path of $\mathrm{TMGa} / \mathrm{H}_{2}$ mixtures. In figure 3.11 we plot the ratio of the $\mathrm{CH}_{4}$ present at each temperature to the TMGa concentration loss (TMGa) due to decomposition at that temperature. This ratio gives a measure of the number of methyl groups lost from the TMGa in the decomposition process. For low temperatures, where there is quantifiable loss of $\mathrm{TMGa}$, the ratio $\mathrm{R}=\mathrm{CH}_{4} / \Delta \mathrm{TMGa}=2$, indicates that more than one methyl group is lost from the TMGa prior to sampling of the gas. We have observed a range of values for this low temperature ratio, $R$, e.g. $1.7 \leq R \leq 2.0$. These data suggest suggest that the loss of the first methyl group from the TMGa is followed promptly by the loss of a second methyl group at a rate that exceeds our current measurement ability. At temperatures above $460^{\circ} \mathrm{C}$ we consistently observe three methane molecules for each decomposed TMGa molecule. This observation suggests that, contrary to the results of Jacko and Price, all methyl radicals are removed from $\mathrm{TMGa}$ in $\mathrm{H}_{2}$ at temperatures above $500^{\circ} \mathrm{C}$. 


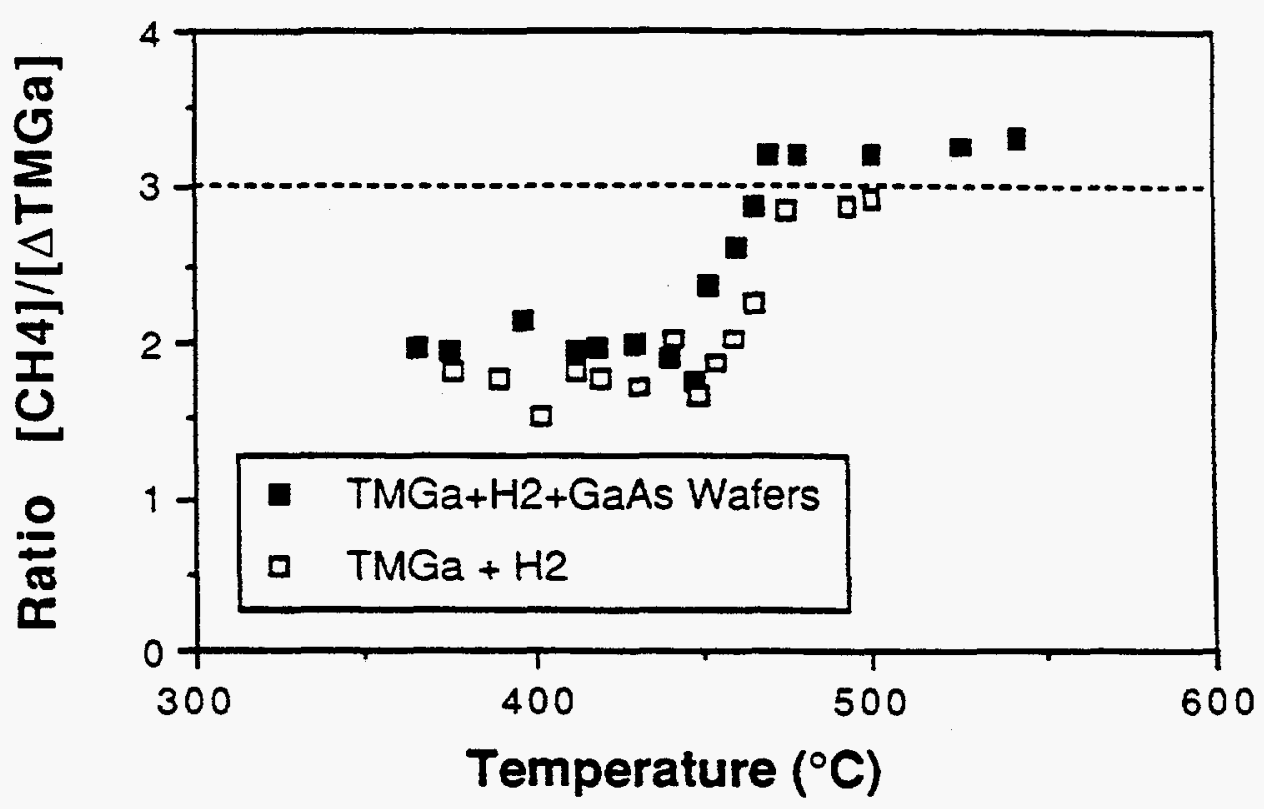

Figure 3.11

Ratio of evolved $\mathrm{CH} 4$ concentration to change in concentration of TMGa. The low temperature value of $R$ is 2.0 , while above $500^{\circ} \mathrm{C}, \mathrm{R} \sim 3$

It appears that the fission of methyl groups on TMGa occurs in stages. The first group is removed by homogeneous reactions. This is suggested by the relative insensitivity of the reaction rate constant and its temperature variation to reactor surfaces and deposits. The loss of the second methyl group cannot be measured by our current experimental arrangement. The variation of $R$ at low temperature for different runs and conditions suggests that factors other than homogeneous reaction can play a role.

We are not able to reliably extract the reaction rates for the loss of the second and third methyl group. However, the rate of loss of the 
second methyl group is at least as large as that for the first methyl group. The sharp transition between the temperature region where the two and three methyl groups are lost suggests a high activation energy for this process. A more accurate determination of the rate constants than is possible with the current apparatus is required to assess the degree of decomposition of TMGa in the boundary layer of a typical MOCVD reactor.

The decomposition of $\mathrm{AsH}_{3}$ in $\mathrm{H}_{2}$ is characterized by an activation energy of $34 \mathrm{kcal} / \mathrm{mol}$ when analyzed as a first order reaction, see figure. 3.12. This activation energy is somewhat larger than that determined by Tamaru 8 (23 kcal/mol). In Tarmaru's work, however, the decomposition was carried out on coherent arsenic and antimony surfaces at very low temperatures. Our data suggest that $\mathrm{AsH}_{3}$ decomposition is largely surface catalyzed. This is indicated by the sensitivity of decomposition rate to the surface preparation and wall deposits. An example of the effect of GaAs surfaces on the rate of decomposition of $\mathrm{AsH}_{3}$ is also shown in figure 3.12 . The activation energy for the process is $18 \mathrm{kcal} / \mathrm{mol}$ in the temperature range below $\sim 530^{\circ} \mathrm{C}$ when GaAs surfaces are present and increases above $530^{\circ} \mathrm{C}$ to the value determined for homogeneous or quartz decomposition ( $34 \mathrm{kcal} / \mathrm{mol}$ ). This transition suggests that homogeneous or quartz catalyzed decomposition dominates at temperatures above $530^{\circ} \mathrm{C}$ in our experiments. 


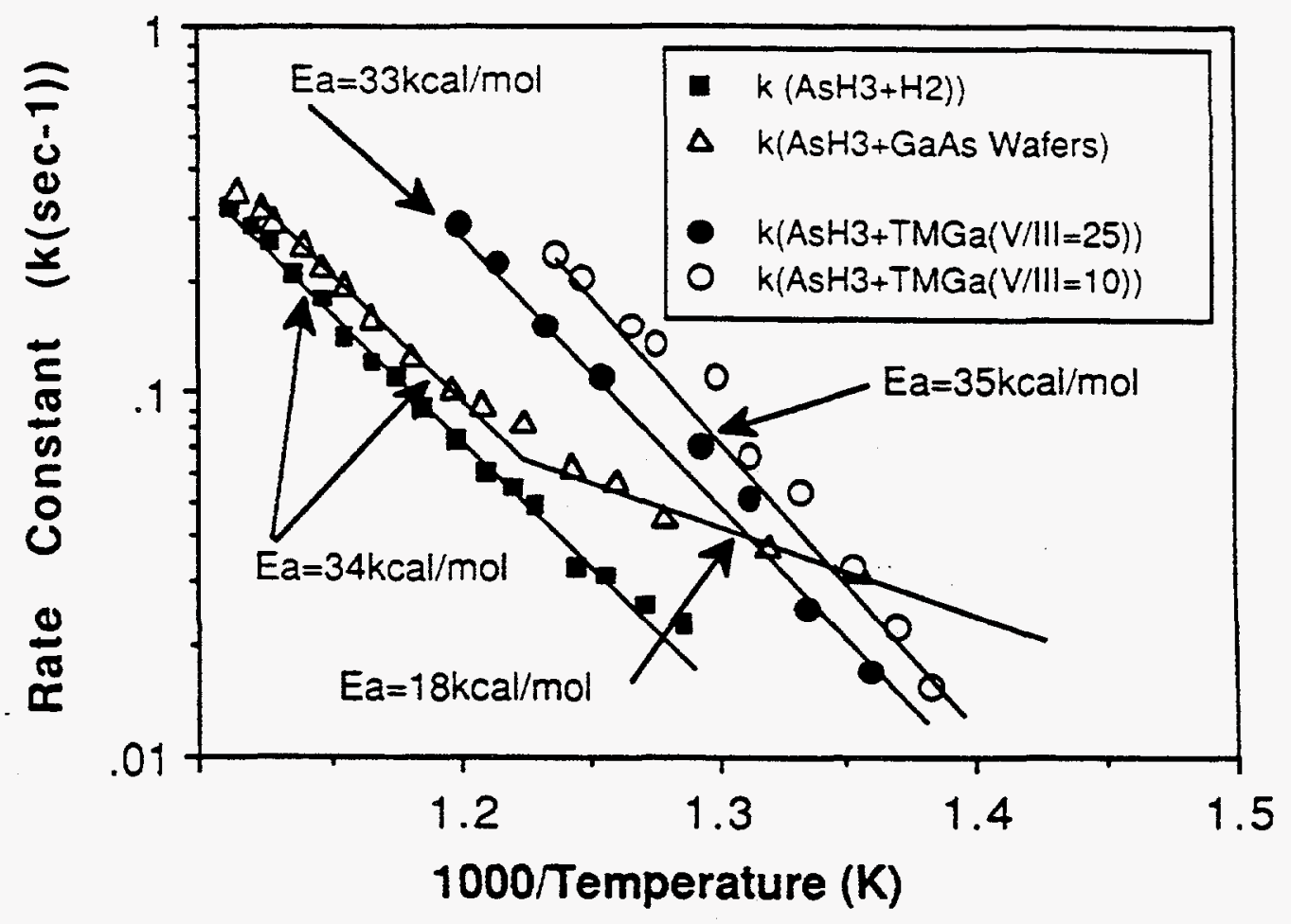

Figure 3.12

Effective rate constant for decomposition of arsine in $\mathrm{H}_{2}, \mathrm{H}_{2}+\mathrm{TMGa}$, and $\mathrm{H}_{2}$ with $\mathrm{GaAs}$ (100) wafers present.

The presence of TMGa in the reactor greatly enhances the rate of decomposition of $\mathrm{AsH}_{3}$, but does not substantially alter the activation energy of the process. A complete explanation for this is not possible at present. The implication for the growth of GaAs however is that TMGa promotes the decomposition of $\mathrm{AsH}_{3}$ at temperatures well below normal growth temperature. Clearly this process dominates the decomposition of arsine for most MOCVD growth conditions. 


\subsection{Sampled Gas IR Spectroscopy DEAsH/TEAs}

In order to achieve thermally driven ALE of III- $\mathrm{V}$ compounds a low decomposition temperature source of arsenic would be beneficial. Also, reducing the safety hazards associated with arsine gas by development of high purity organometallic group $V$ sources would be of considerable importance to growth of gallium arsenide by MOCVD. The use of liquid organometallic arsenic sources would reduce the toxic hazard and handling problems associated with high pressure arsine gas cylinders. The group $\mathrm{V}$ hydrides also have slow decomposition rates and require high temperatures for pyrolysis. Thus, large excesses of arsine are required to obtain good epitaxial growth. In contrast, group V organometallics sources might potentially decompose at temperatures allowing a more stoichiometric mixture in the gas phase at "normal" growth temperatures or they may allow reduced temperature growth to eliminate temperature driven diffusion of dopants. These benefits have led to the investigation of alternative arsenic sources to replace arsine. $9,10,11,12$ However, little is currently understood about the basic chemical reactions operative in the MOCVD growth of GaAs with these new organoarsenic sources.

In this study we present data on the reaction mechanisms and rates of decomposition of the organometallic $\mathrm{V}$ sources triethylarsenic (TEAs) and diethylarsine (DEAsH) in a hydrogen carrier gas. In addition, the catalytic reactivity of single crystal GaAs surfaces on the decomposition of the gaseous reactants is also studied. 


\subsubsection{IR Spectra DEAsH/TEAs}

The infrared absorption spectra of $\mathrm{DEAsH} / \mathrm{H}_{2}$, and $\mathrm{TEAs} / \mathrm{H}_{2}$ mixtures sampled from a flowing gas stream have been examined in detail at various temperatures. The room temperature spectra of DEAsH and TEAs is shown in figure 3.13 and figure 3.14 respectively. For our current experiments we have monitored the IR absorption peaks at 2980 , 2080,955 and $585 \mathrm{~cm}^{-1}$, which correspond to the $\mathrm{C}-\mathrm{H}$, As-H vibrational modes and the $\mathrm{C}=\mathrm{C}$, As- $\mathrm{C}$ stretching modes, respectively. ${ }^{5}$ It is of interest to note that the As-H vibrational mode in the DEAsH molecule is located at $2080 \mathrm{~cm}^{-1}$, which is red shifted with respect to its value of $2122 \mathrm{~cm}^{-1}$ in the arsine molecule. Based upon this observation we conclude that the appearance of an unknown peak at $2080 \mathrm{~cm}^{-1}$ previously observed in decomposition studies of the $\mathrm{TMGa} \mathrm{AsH}_{3}$ system $^{6}$ is likely to be caused ty the formation of an intermediate organoarsenic compound. These compounds may play a role in conventional MOVPE growth of GaAs. 


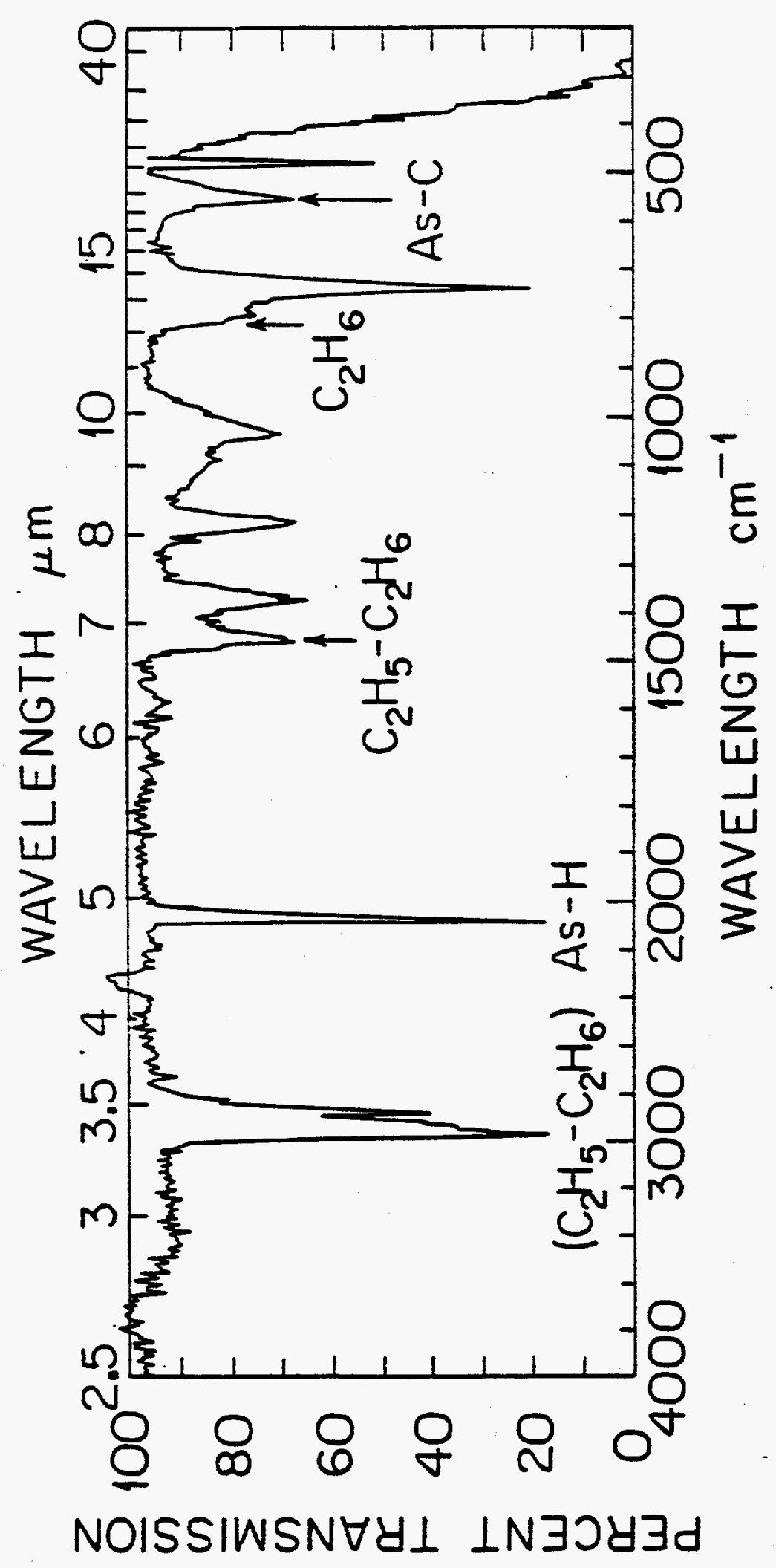




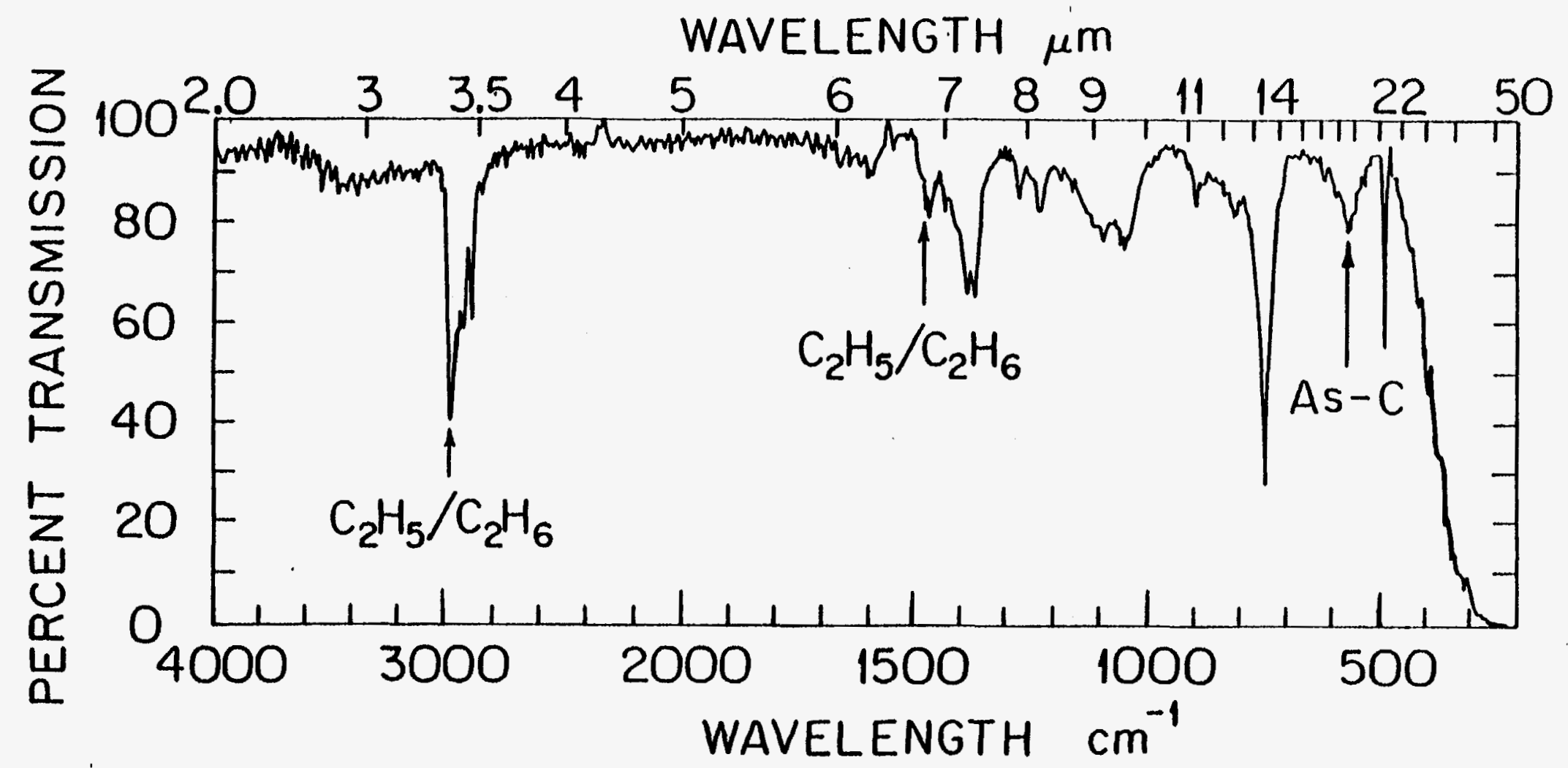

Figure 3.14

Infrared absorption spectrum of triethylarsenic at room temperature 


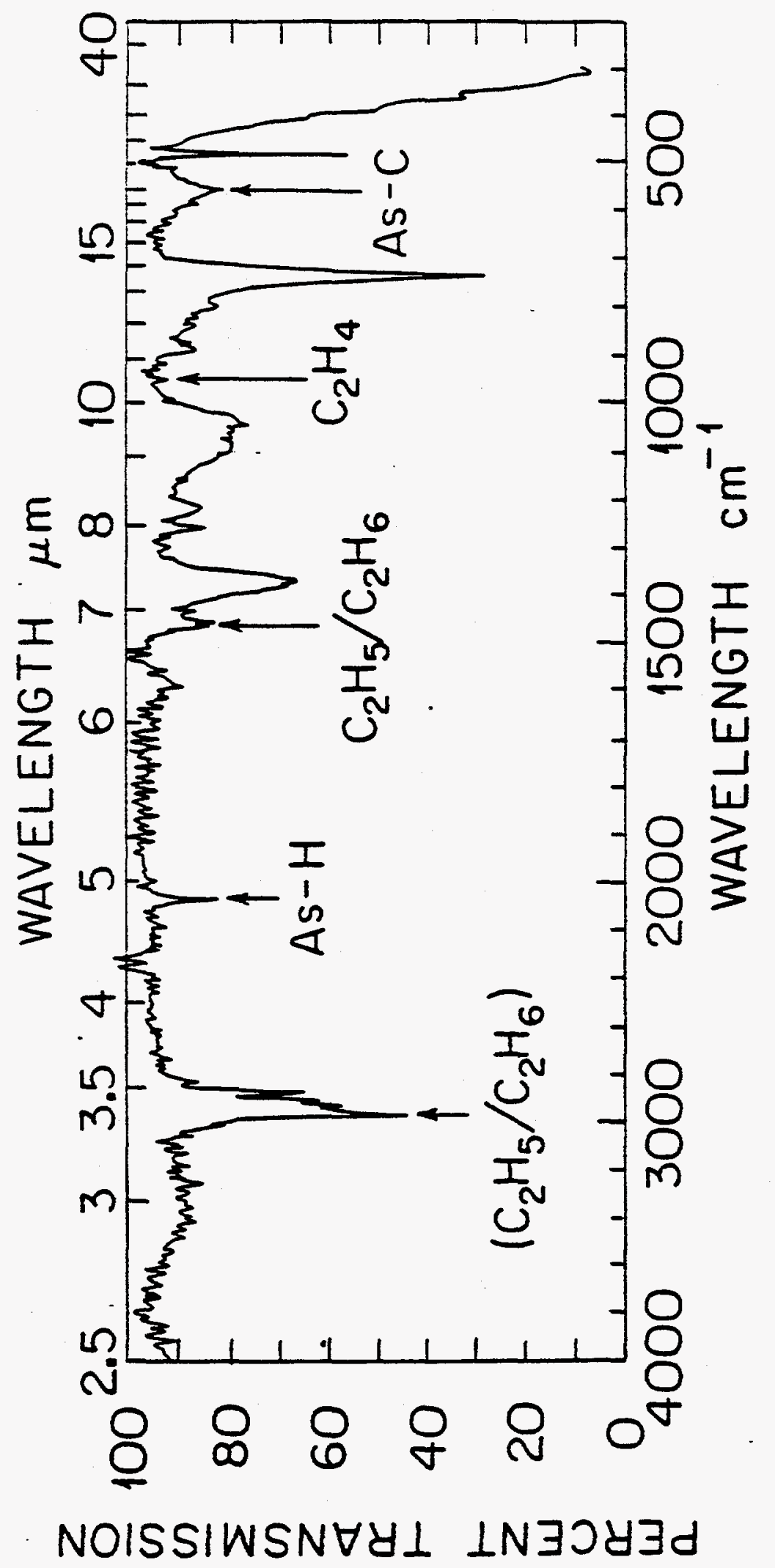




\subsubsection{DEAs/TEAs Reaction Mechanism}

Upon thermally decomposing the DEAsH, ethane $\left(\mathrm{C}_{2} \mathrm{H}_{6}\right)$ and ethylene $\left(\mathrm{C}_{2} \mathrm{H}_{4}\right)$ are observed to be the main reaction products. In addition, a small quantity of arsine at $2122 \mathrm{~cm}^{-1}$ was observed to be produced at temperatures exceeding $440^{\circ} \mathrm{C}$. From infrared spectral analysis it was determined that the pyrolysis of DEAsH begins at $400^{\circ} \mathrm{C}$ and is virtually complete at $460^{\circ} \mathrm{C}$. In a hydrogen carrier stream the decomposition reaction for DEAsH appears to be:

$$
\left(\mathrm{C}_{2} \mathrm{H}_{5}\right)_{2} \mathrm{AsH}-\cdots>1 / 2 \mathrm{As}_{2}+(2-\mathrm{x}) \mathrm{C}_{2} \mathrm{H}_{6}+\mathrm{xC}_{2} \mathrm{H}_{4}
$$

The value of $\mathrm{x}$ for this reaction can not be quantitatively determined since the absolute vapor pressure of $\mathrm{DEAsH}$ at $20^{\circ} \mathrm{C}$ is unknown.

Triethylarsenic shows a different reaction pathway, which interestingly enough yields $\mathrm{DEAsH}$ as an intermediate reaction product in the temperature range of $350-430^{\circ} \mathrm{C}$. Figure 3.15 shows an infrared absorption spectrum of TEAs taken at $410^{\circ} \mathrm{C}$, note the appearance of the As- $\mathrm{H}$ vibrational mode at $2080 \mathrm{~cm}^{-1}$. A ß-hydride elimination mechanism is the most likely reaction pathway that explains the formation of DEAsH from the thermal decomposition of TEAs. This can be described by the following expression:

$$
\left(\mathrm{C}_{2} \mathrm{H}_{5}\right)_{3} \mathrm{As}--->\left(\mathrm{C}_{2} \mathrm{H}_{5}\right)_{2} \mathrm{AsH}+\mathrm{C}_{2} \mathrm{H}_{4}\left(350-430^{\circ} \mathrm{C}\right)
$$


Figure 3.16 shows the relative concentration of the gaseous species as a function of temperature in the hot zone. Since the vapor pressure of DEAsH is not known, the concentration of DEAsH was estimated by taking the IR absorptivity at the As- $\mathrm{H}$ vibrational frequency to be $1 / 3$ of the value for the arsine molecule. Note that the evolution of DEAsH increases until a temperature of $430^{\circ} \mathrm{C}$ at which point the rate of decomposition of DEAsH exceeds the rate of its formation from the decomposition of TEAs. On account of the overlap in the ethyl and ethane IR absorption peaks the relative concentration of $\mathrm{C}_{2} \mathrm{H}_{6}$ is not plotted.

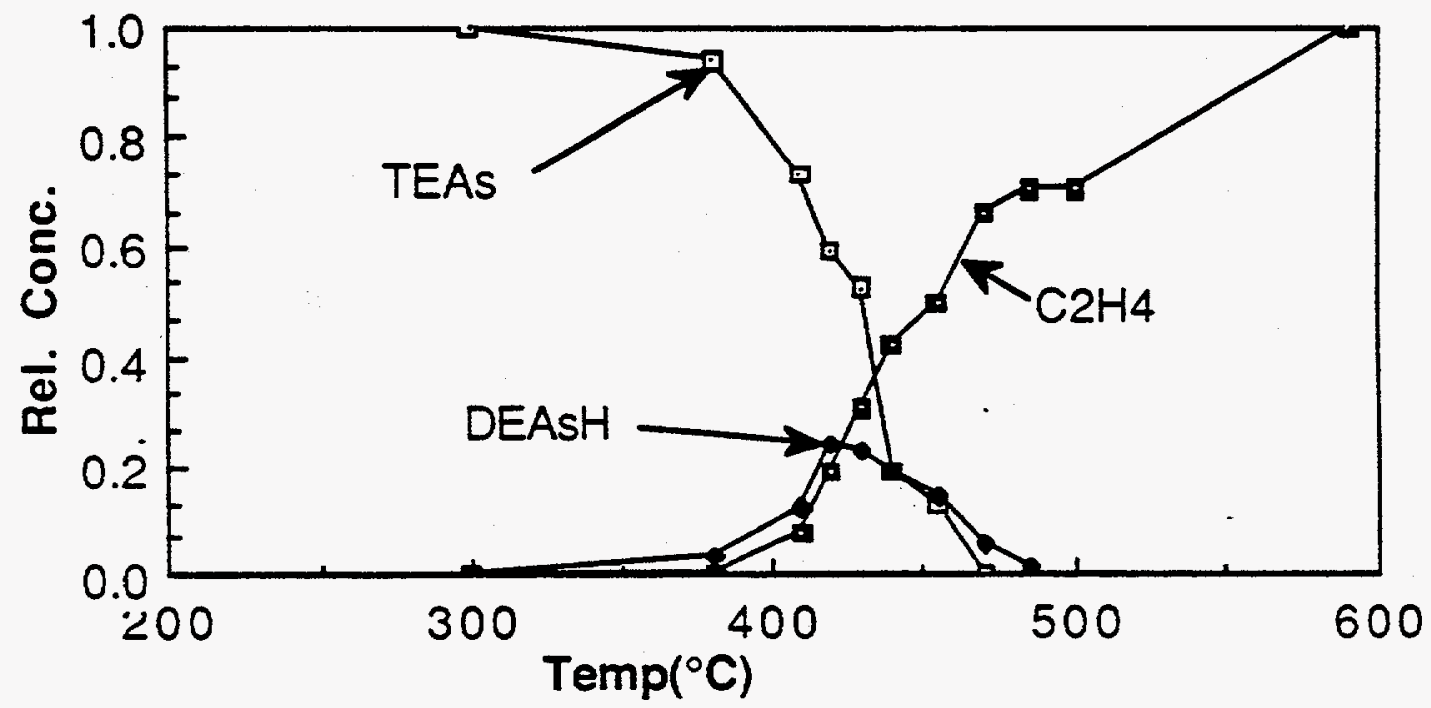

Figure 3.16.

Relative concentration of triethylarsenic, diethylarsine and ethylene and as a function of temperature.

The addition of $\operatorname{GaAs}(100)$ wafers to the hot zone has been found to catalyze the decomposition of both DEAsH and TEAs. As shown in figure 
3.17 the relative rates of decomposition of both DEAsH and TEAs at $430^{\circ} \mathrm{C}$ are 3 times greater with $\mathrm{GaAs}$ surfaces in the hot zone. In addition, the slopes of the decomposition curves are steeper upon the addition of GaAs wafers which is indicative of a different activation energy for the process.

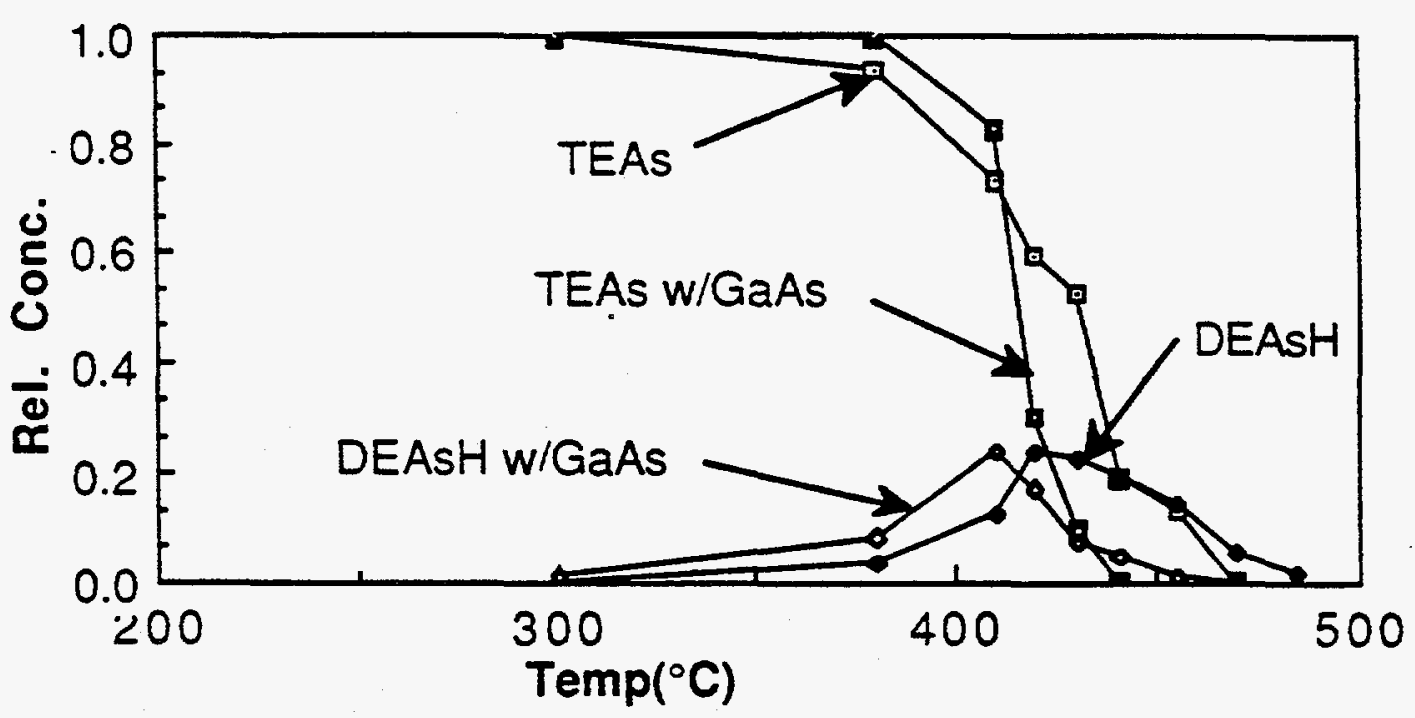

Figure 3.17

Effect of $\mathrm{GaAs}(100)$ surfaces on the pyrolysis of TEAs and DEAsH.

\subsubsection{DEAsH/TEAs Activation Energies}

For the simple uncatalyzed pyrolysis of DEAsH and TEAs we model the decomposition by first order reaction kinetics. As shown in figure 3.18 , an activation energy of $43.3 \mathrm{kcal} / \mathrm{mole}$ was measured for the decomposition rate of TEAs. This was determined by monitoring the As- 
C stretching frequency $\left(565 \mathrm{~cm}^{-1}\right)$ in the TEAs molecule. The decomposition of DEAsH exhibited a significantly lower activation energy of $20.6 \mathrm{kcal} / \mathrm{mole}$ for the $\mathrm{As}-\mathrm{H}$ vibrational frequency $(2080 \mathrm{~cm}-1)$, see figure 3.19. The activation energy for the decomposition of DEAsH is significantly lower than the As- $\mathrm{H}$ bond strengths 13 in $\mathrm{AsH}_{3}$, similar to results of earlier studies on the decomposition of $\mathrm{AsH}_{3} .^{14}$

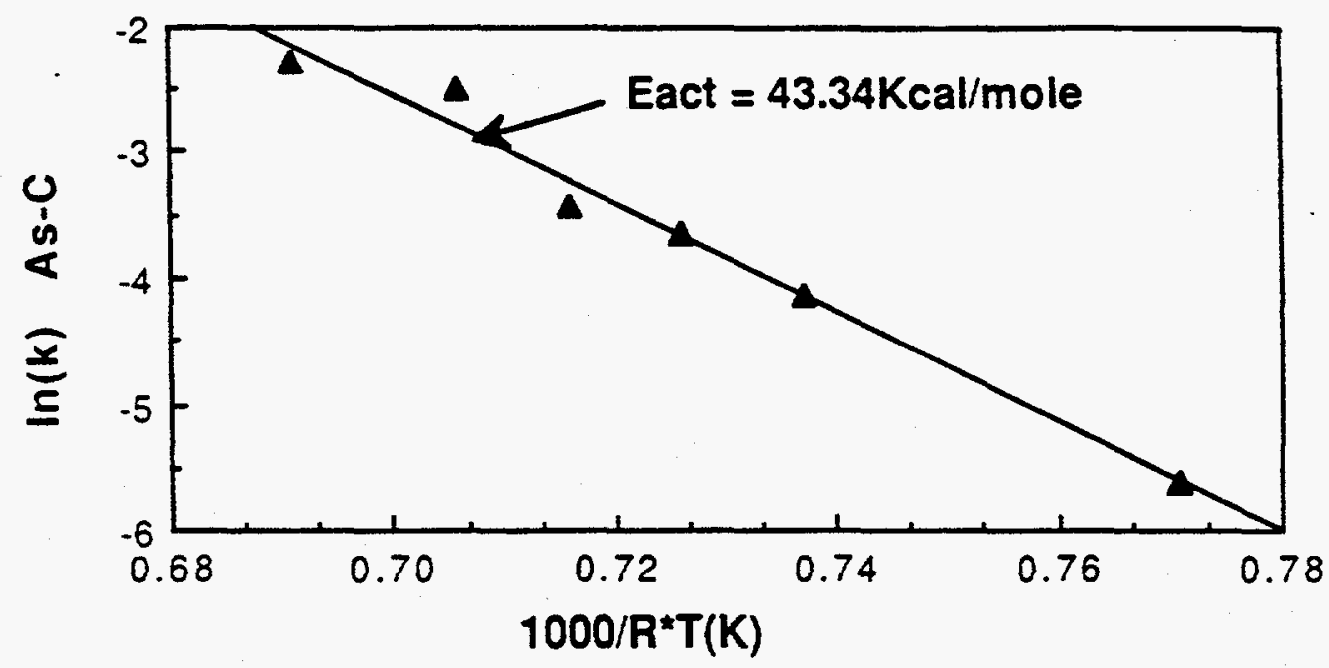

Figure 3.18.

Reaction rate of triethylarsenic plotted against inverse temperature times ideal gas constant yields activation energy of decomposition process. 


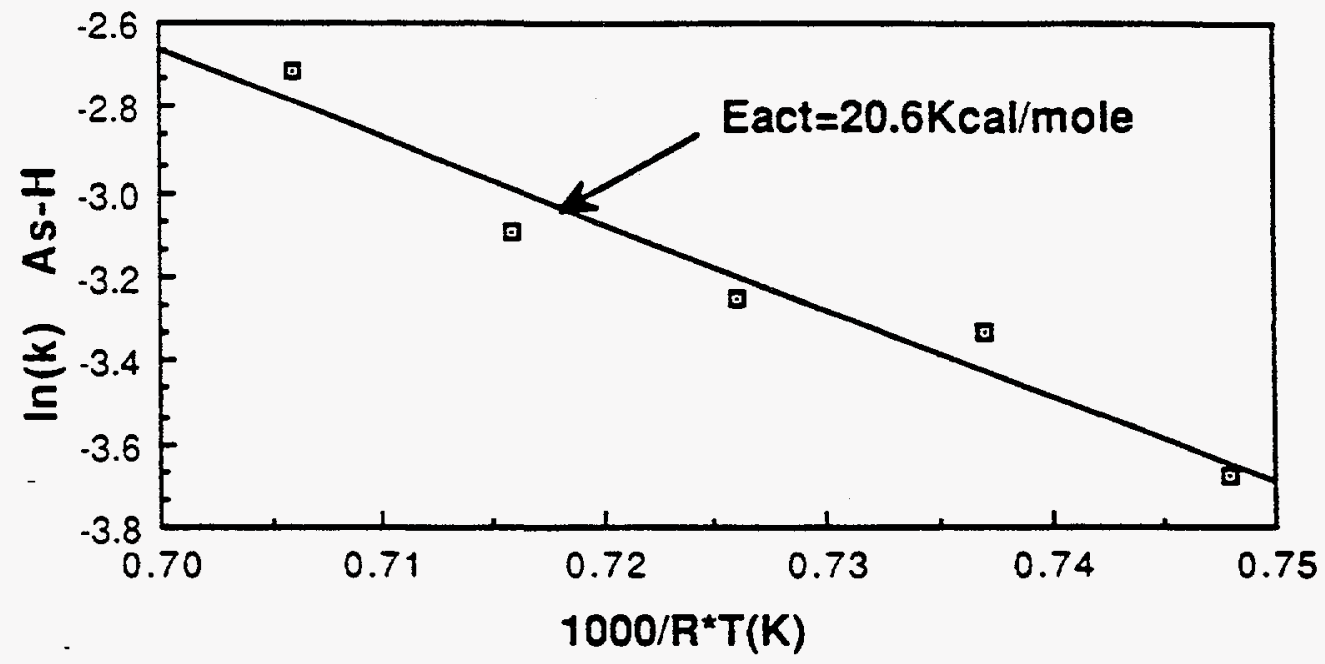

Figure 3.19.

Reaction rate of diethylarsine as a function of inverse temperature.

Table 3.1 Rate Constants for DEAsH/TEAs Decomposition

\begin{tabular}{|ccc|}
\hline \multicolumn{3}{|c|}{$k=\mathrm{A} \exp \left[-\mathrm{E}_{\mathrm{a}} / \mathrm{RT}\right]$} \\
\hline & $\log \mathbf{1 0} \mathbf{A}\left(\mathbf{s e c}^{-1}\right)$ & $\mathbf{E}_{\mathbf{a c t}}(\mathbf{k c a l} / \mathbf{m o l})$ \\
\hline $\mathrm{TEAs} / \mathrm{H} 2$ & 12.1 & 43.4 \\
\hline $\mathrm{DEAsH}$ & 5.1 & 20.6 \\
\hline
\end{tabular}

\subsubsection{TEAs Decomposition Kinetics Model}

By using the effective reaction rate constants shown in Table 3.1, we can generate a kinetic model which supports the experimentally observed evolution of $\mathrm{DEAsH}$ and $\mathrm{C}_{2} \mathrm{H}_{4}$ from the decomposition of TEAs by a $\beta$-hydride elimination mechanism. The temperature dependence of the relative concentration of $\operatorname{TEAs}\left(\mathrm{C}_{\mathrm{f}} / \mathrm{C}_{0}\right)$ at a fixed contact time is shown 
in figure 3.20 , in which both the line representing the kinetic model equation 3.23 and experimentally measured points are shown.

$$
\operatorname{TEAs}\left(\frac{\mathrm{C}_{f}}{\mathrm{C}_{0}}\right)=\exp \left(-\mathrm{k}_{1} \tau\right)
$$

If we assume that all the TEAs decomposes to form DEAsH then the relative amount evolved should be $\left(1-\mathrm{C}_{\mathrm{f}} / \mathrm{C}_{\mathrm{o}}\right)$. By using the effective rate constant for the decomposition of DEAsH observed separately in the DEAsH experiment we can write the following kinetic expression for the final DEAsH concentration observed:

$$
\operatorname{DEAsH}\left(\frac{\mathrm{C}_{\mathrm{f}}}{\mathrm{C}_{\mathrm{o}}}\right)=\left[1-\exp \left(-\mathrm{k}_{1} \tau\right)\right] * \exp \left(-\mathrm{k}_{2} \tau\right)
$$




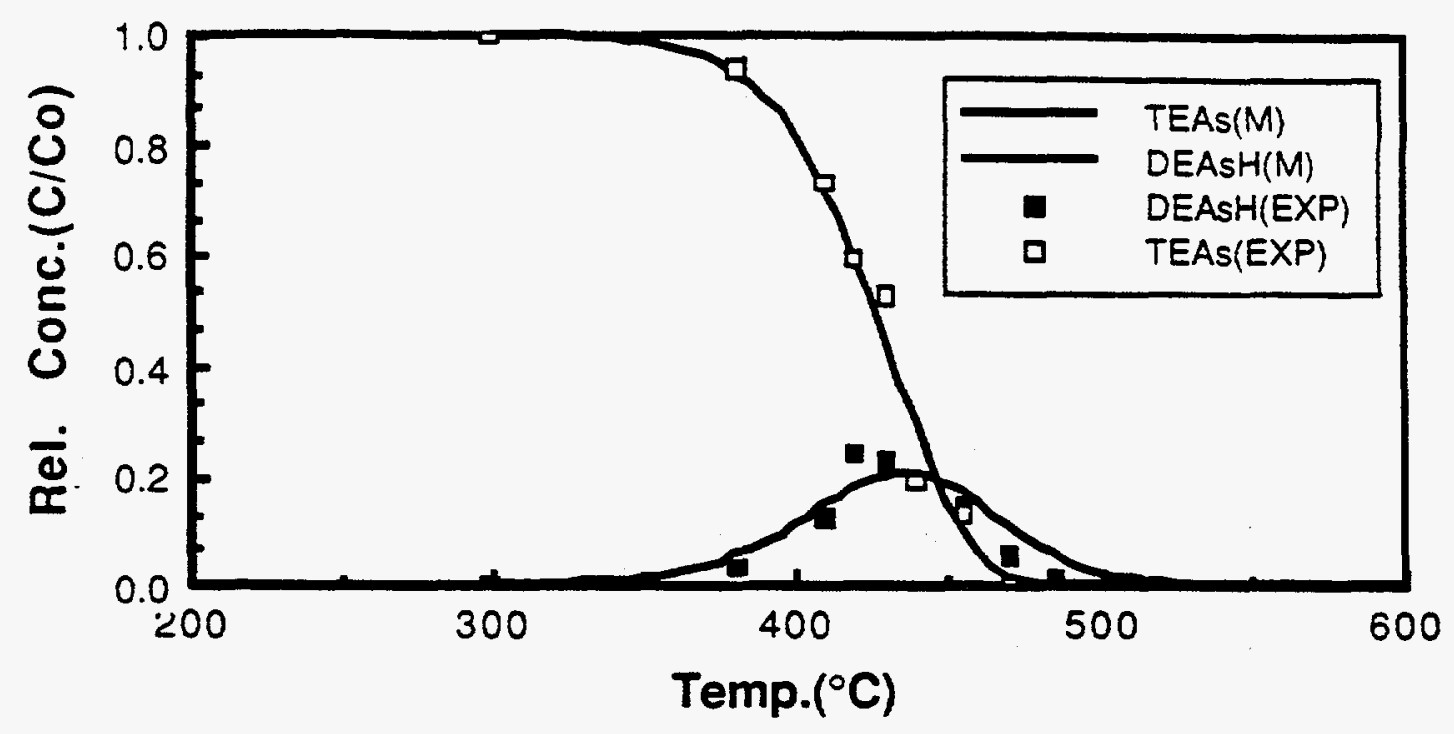

Figure 3.20.

Fit of reaction kinetics model to the experimentally observed relative concentrations of TEAs and DEAsH.

As shown in Fig. 3.20, there is an excellent fit of the experimental data to the kinetic model. This supports the conclusion that the predominant mechanism for TEAs decomposition is $\beta$-hydride elimination resulting in the formation of DEAsH and ethylene. Consequently, it appears that DEAsH is the more stable organoarsenic molecule.

This is also supported by the experimental observation that upon the addition of trimethylgallium (TMGa) to gas stream a low volatility liquid adduct forms with TEAs, but not with DEAsH. The formation of the TEAs/TMGa adduct has required heating of reactor lines to $120^{\circ} \mathrm{C}$ in 
order to obtain successful GaAs growth. ${ }^{15}$ The use of DEAsH in MOCVD growth has yielded reasonably high purity GaAs with n-type background free carrier concentrations as low as $3 \times 10^{14} \mathrm{~cm}^{-3}$ and $77 \mathrm{~K}$ mobility of $64,600 \mathrm{~cm}^{2} /(\mathrm{V} \cdot \mathrm{sec})$. On the basis of our studies and previous work, we conclude that DEAsH is a most promising arsine replacement. In addition, the As to hydrogen bond in DEAsH may play an important role in reducing the carbon incorporation in GaAs by hydrogenolysis of the methyl groups from TMGa. This view is supported by the work of $R$. Bhat 16 who observed high p-type background GaAs layers when elemental arsenic was substituted for arsine.

\subsection{Summary}

By using sampled gas infrared absorption spectroscopy we have been able to effectively measure the decomposition rates for the $\mathrm{TMGa} / \mathrm{AsH}_{3} / \mathrm{H}_{2}$ system. Table 3.2 summarizes first order rate constant for the MOCVD process. The pyrolysis of TMGa was found to occur in stages, with the first two methyl groups releasing in the $350-450^{\circ} \mathrm{C}$ range. It is not definite that all stages of alkyl elimination are homogeneous. However, the rate of decomposition is not enhanced by wall deposits of GaAs substrates for the TMGa partial pressure and reactor pressure examined. The only gas phase by product of the decomposition observed is $\mathrm{CH}_{4}$. Arsine decomposition on the other hand is strongly catalyzed by wall deposits, GaAs surfaces, and gaseous mixtures with TMGa. The activation energy for $\mathrm{AsH}_{3}$ decomposition is reduced for the reactor at the GaAs surface. We emphasize that these catalytic reactions play an 
important role in the MOCVD growth of GaAs and must be taken into account when modeling a large scale reactor.

Thermal decomposition of TEAs in $\mathrm{H}_{2}$ occurs via a B-hydride elimination mechanism, producing DEAsH and $\mathrm{C}_{2} \mathrm{H}_{4}$ as the organic byproducts. Thermal decomposition of DEAsH was observed to produce predominantly $\mathrm{C}_{2} \mathrm{H}_{6}, \mathrm{C}_{2} \mathrm{H}_{4}$ and a small quantity of arsine. Both DEAsH and TEAs decomposition was discovered to be catalyzed by the addition of single crystal GaAs(100) surfaces to the reactor. Since DEAsH does not form an adduct with TMGa, whereas TEAs does, DEAsH is concluded to be the more attractive group $\mathrm{V}$ alternative source

Table 3.2 Rate Constants for $\mathrm{TMGa}_{\mathrm{AsH}}$ Decomposition

\begin{tabular}{|lcc|}
\hline \multicolumn{3}{|c|}{$k=A \exp \left[-E_{a} / R T\right]$} \\
\hline & $\log 10 \mathrm{~A}\left(\sec ^{-1}\right)$ & $E_{\text {act }}(\mathbf{k c a l} / \mathbf{m o l})$ \\
\hline $\mathrm{TMGa}_{2} \mathrm{H}_{2}$ & 16.64 & 58 \\
\hline $\mathrm{TMGa} \mathrm{w} / \mathrm{GaAs}$ & 18.12 & 62 \\
\hline $\mathrm{AsH}_{3} / \mathrm{H}_{2}$ & 7.55 & 34 \\
\hline $\mathrm{AsH}_{3} / \mathrm{H}_{2} / \mathrm{GaAs}$ & 3.72 & $18\left(\mathrm{~T}<530^{\circ} \mathrm{C}\right)$ \\
\hline $\mathrm{AsH}_{3} / \mathrm{TMGa}(10: 1)$ & 8.86 & $35\left(\mathrm{~T}>530^{\circ} \mathrm{C}\right)$ \\
\hline $\mathrm{AsH} / \mathrm{TMGa}(25: 1)$ & 8.13 & 33 \\
\hline
\end{tabular}




\section{REFERENCES-CHAPTER 3}

1 I. R. Levine, Physical Chemistry, (McGraw-Hill Publ. Co.,New York, 1978) p. 771.

2 S. W. Benson, Thermochemical Kinetics, 2nd Ed, (John Wiley \& Sons, New York, 1976) p. 17.

3 S. W. Benson, Thermochemical Kinetics, 2nd Ed, (John Wiley \& Sons, New York, 1976) p. 85.

4 M. Yoshida, H. Watanabe, and F. Uesugi, J. Electrochem. Soc. 130, 413 (1985).

5 M.R. Leys and H. Veenvliet, J. Crystal Growth, 55, 145 (1981).

6 J. Nishizawa and T. Kurabayashi, J. Electrochem. Soc. 130, 413 (1983).

7 M. G. Jacko, and S. J. W. Price, Can. J. Chem. 41, 1560 (1963).

$8 \quad$ K. Tamura, J. Phys. Chem. 59, 777 (1955).

9 P. Lee, D. McKenna, D. Kapur and K.F. Jensen, J. Crystal Growth. 77,120 (1986).

10 W.T. Tsang, Appl. Phys. Lett. 45,1234 (1984).

11 R. Bhat, M.A. Koza, and B.J. Skromme, Appl. Phys. Lett. 50, 1194 (1987).

12 D.M. Speckman and J.P. Wendt, Appl. Phys. Lett. 50, 676 (1987).

13 S.R. Gunn, Inorg. Chem., 11, 796 (1972). 
14 S.P. DenBaars, B.Y. Maa, P.D. Dapkus, A.D. Danner and H.C. Lee, J. Crystal Growth.77, 188 (1986).

15 K. Tamaru, J. Phys. Chem. 59, 777 (1955).

16 R. Bhat, J. Electron. Mater. 14, 433 (1985). 


\section{CHAPTER 4}

\section{ATOMIC LAYER EPITAXY BY THERMALLY DRIVEN METALORGANIC CHEMICAL VAPOR DEPOSITION}

\subsection{Introduction}

As described in the first chapter, the Atomic Layer Epitaxy (ALE) process is a stepwise deposition process in which alternate monolayers of each constituent element in the compound are deposited using a selflimiting surface reaction mechanism. By employing a new regime of MOCVD growth, in which saturated surface reactions control the growth, it is possible to alternately deposit monolayers of column III and column $\mathrm{V}$ elements so that only one monolayer of the III-V compound semiconductor is deposited in every cycle of the deposition. Extremely uniform films can be deposited by repetition of this alternate exposure cycle. For III-V compounds, the use of a volatile molecule for the column III element is required to avoid clustering of the element on the surface. In this study, we employ conventional MOCVD reactants trimethylgallium (TMGa), trimethylaluminum (TMAl), and arsine $\left(\mathrm{AsH}_{3}\right)$ as the $\mathrm{ALE}$ reactants for the growth of GaAs, AlAs and AlGaAs. We describe the kinetics of thermally driven ALE growth using these reactants in both $\mathrm{H}_{2}$ and $\mathrm{N}_{2}$ ambients in the temperature range $445^{\circ} \mathrm{C}$ $500^{\circ} \mathrm{C}$. We further demonstrate that this technique can be integrated with the existing technological base of conventional MOCVD. Hybrid structures are grown by utilizing ALE for the layers of critical thickness 
control and thicker layers by MOCVD. In order to integrate the two technologies several design considerations must be addressed. This chapter describes the ALE system employed in this work, saturated growth results, and kinetic growth model.

\subsection{Design of ALE Reactor}

ALE was performed in a modified atmospheric MOCVD reactor system. The system was designed to allow fast transitions between the column III and column $\mathrm{V}$ gas sources with a minimum of intermixing. Figure 4.1 shows a schematic of the modified vent/run MOCVD system which is equipped with a pressure balanced injection manifold to eliminate gas concentration fluctuations. The reactor vessel is a small volume rectangular tube which is water-cooled on the top surface to promote laminar gas flow. The average gas residence time in this reactor design is only 0.5 seconds at the high flowrates employed in this work $(35 \mathrm{~cm} / \mathrm{sec})$. The recessed graphite susceptor lies flush with the adjacent bottom quartz wall insuring minimal gas turbulence along the leading edge. The small residence time growth chamber allowed alternate exposures of the substrate to be achieved by a pulsed gas injection method. In this method, ALE of GaAs, AlAs, and AlGaAs is achieved by using conventional MOCVD precursors in a four step gas injection sequence at low growth temperatures.

The use of variable growth temperature in a run allowed us to grow the best quality GaAs/AlGaAs heterostructure by a hybridization of ALE and conventional MOCVD. By using an infrared heating assembly rapid 
and conventional MOCVD. By using an infrared heating assembly rapid changes in the growth temperature were possible. The highest quality AlGaAs is grown by conventional MOCVD at $750^{\circ} \mathrm{C} .1$ The temperature is then lowered to the surface controlled growth regime $\left(445-500^{\circ} \mathrm{C}\right)$ where the quantum well active regions are grown by ALE. 


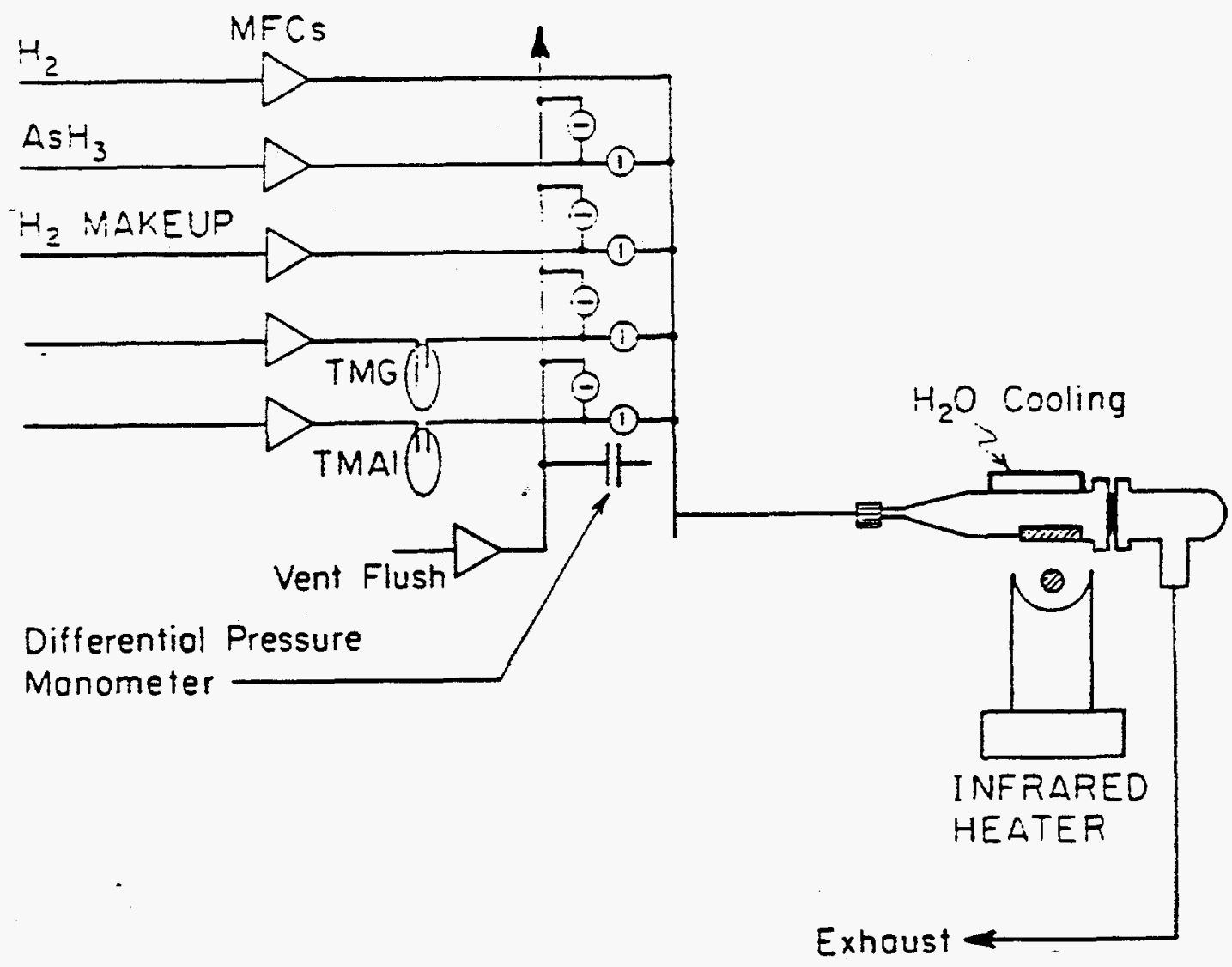

Figure 4.1

Schematic of fast gas switching ALE system. Note pressure-balancing of vent/run lines to minimize gas phase mixing of gaseous precursors. 


\subsection{Experimental Procedure}

Both undoped and Si-doped GaAs, orientated $(100) \pm 0.5^{\circ}$, are used as substrates. The cleaning procedure consists of a 5 minute heated bath in separate beakers of trichloroethane $(1,1,1)$, acetone, and methanol. Following a 5 minute $18 \mathrm{M} \Omega$ deionized (DI) water rinse, the sample is etched in a 3:1:1 $\left(\mathrm{H}_{2} \mathrm{SO}_{4}: \mathrm{H}_{2} \mathrm{O}_{2}: \mathrm{H}_{2} \mathrm{O}\right)$ solution for 3 minutes to remove polish damage and surface oxides. Finally, the sample is given a 10minute $18 \mathrm{M} \Omega \mathrm{DI}$ water rinse and then blown dry with filtered nitrogen.

The sample is then loaded onto a graphite susceptor in the horizontal reactor. The quartz chamber is connected to the system with glass-to-metal seals via ultra-Torr O-ring fittings (Cajon, Inc.). The reactor is pumped down to 75 millitorr and back-filled twice with ultrapure hydrogen to eliminate residual oxygen from the system. After a 1/2 hour hydrogen purge, the sample is heated to $750^{\circ} \mathrm{C}$ under a arsine overpressure to remove surface oxides. The temperature is monitored with a Alumel-Chromel type $\mathrm{K}$ thermocouple (Omega Inc), which is sheathed in a quartz feedthrough. Calibration of the surface temperature on the graphite susceptor is performed at the Al-Si eutectic point $\left(577^{\circ} \mathrm{C}\right)$. This is accomplished by monitoring the transition in the surface reflection pattern of $1000 \AA \mathrm{Al}$ dots on a Si substrate with a He-Ne laser beam.

Growth is initiated by switching the group III metalorganic from

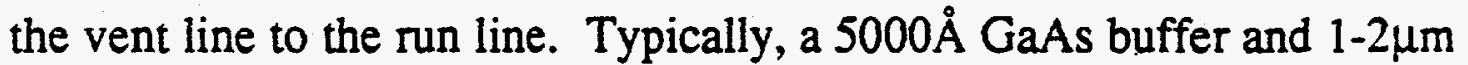
AlGaAs layer are grown by conventional MOCVD at $750^{\circ} \mathrm{C}$. The thick $\mathrm{AlGaAs}$ layer insures that the only luminescence from the subsequent 
ALE GaAs layers would be observed. The reactor temperature is then lowered to the surface kinetically controlled growth regime, $500^{\circ} \mathrm{C}$ or lower. The small thermal mass of the thin graphite susceptor in conjunction with the infrared heating lamp allows rapid $300^{\circ} \mathrm{C}$ temperature changes with stability after only 3 minutes. ALE layers are then grown by a four step pulsed gas injection method.

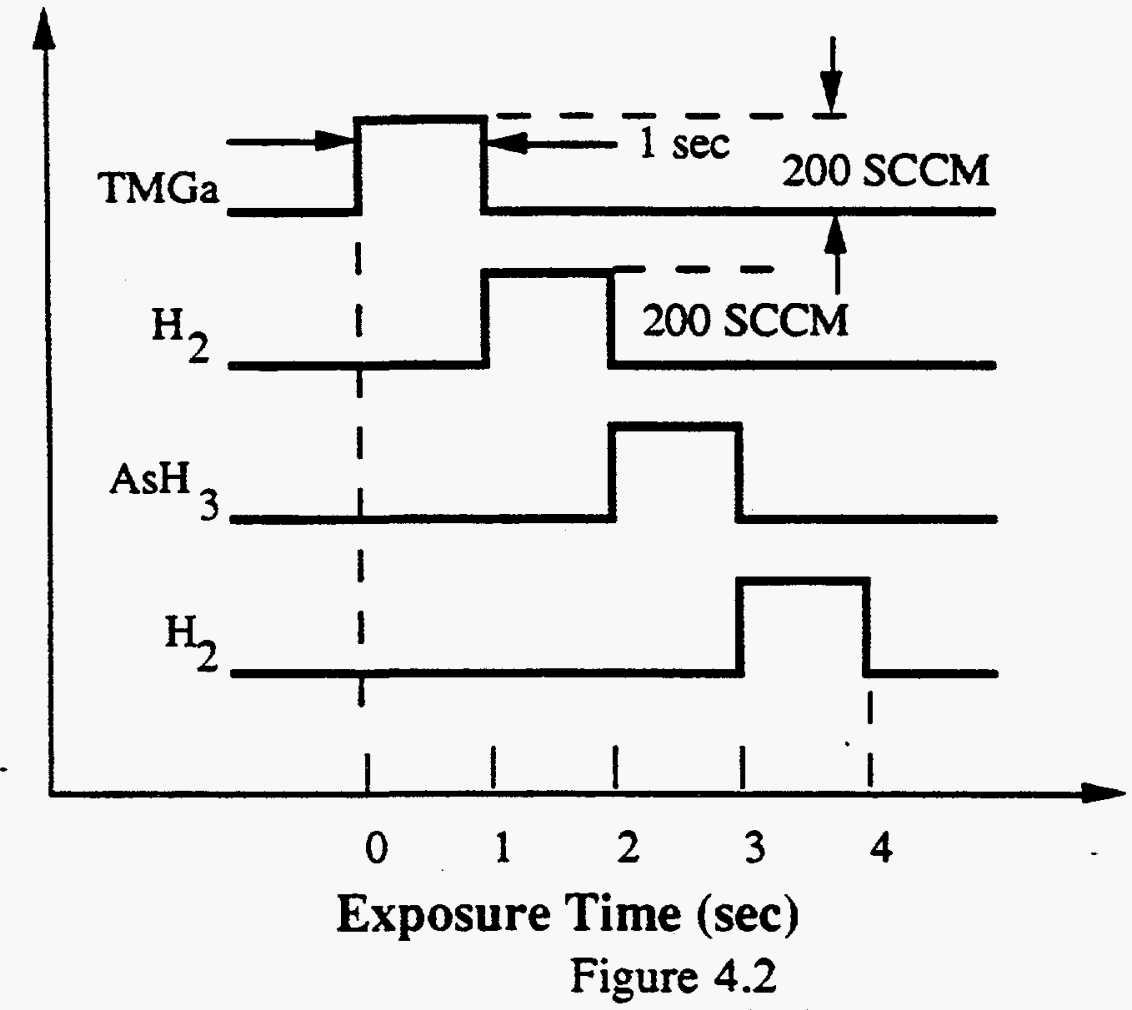

Pulsed injection time sequence in four step ALE growth process

As shown in Figure 4.2, the ALE growth sequence consists of a one second pulse of arsine, a one second pure hydrogen purge, a one second pulse of TMGa or TMAl, and a final one second purge of hydrogen. 
Since the average gas residence time in the growth chamber is only 0.5 seconds at the high gas flow velocities employed in this work, minimal gas phase mixing of the precursors will occur. This has been confirmed by the observation that longer hydrogen purge times did not change the deposited film thickness.

The effect of several growth variables on the ALE process was studied by growing quantum wells under various ALE conditions with the wells separated by $1 \mu \mathrm{m}$ MOCVD-grown AlGaAs barriers. The photoluminescence (PL) from each well could be analyzed to determine material's quality and well thickness. For the Argon ion laser excitation wavelength $(514 \mathrm{~nm})$ utilized in this study the 1/e absorption depth is shorter than $1 \mu \mathrm{m}$, therefore only photoluminescence (PL) emission from the uppermost quantum well is observed. The typical fivt: single quantum well sample (SQW) sample is shown in Figure 4.3. After obtaining PL from the top well, the sample is then etched with a calibrated etch to within $1000 \AA$ of the next quantum well. The etched consisted of a $\mathrm{H}_{2} \mathrm{O}: \mathrm{H}_{2} \mathrm{O}_{2}: \mathrm{H}_{2} \mathrm{SO}_{4}$ mixture in a 80:8:1 ratio. Calibration was confirmed by a surface profiliometer accurate to within $50 \AA$. Utilizing this measurement technique five SQWs of varying growth parameters could be analyzed on the same sample produced in a single run. Layer thicknesses were estimated from peak emission wavelength expected from a Kronig-Penney model of $\mathrm{QW}$ emission energies. A complete explanation of the $\mathrm{QW}$ emission energy model along with a detailed study of the PL from the ALE QWs is presented in Chapter 5. Accuracy of the 
layer thickness determined in this way was confirmed by cleaved corner TEM.

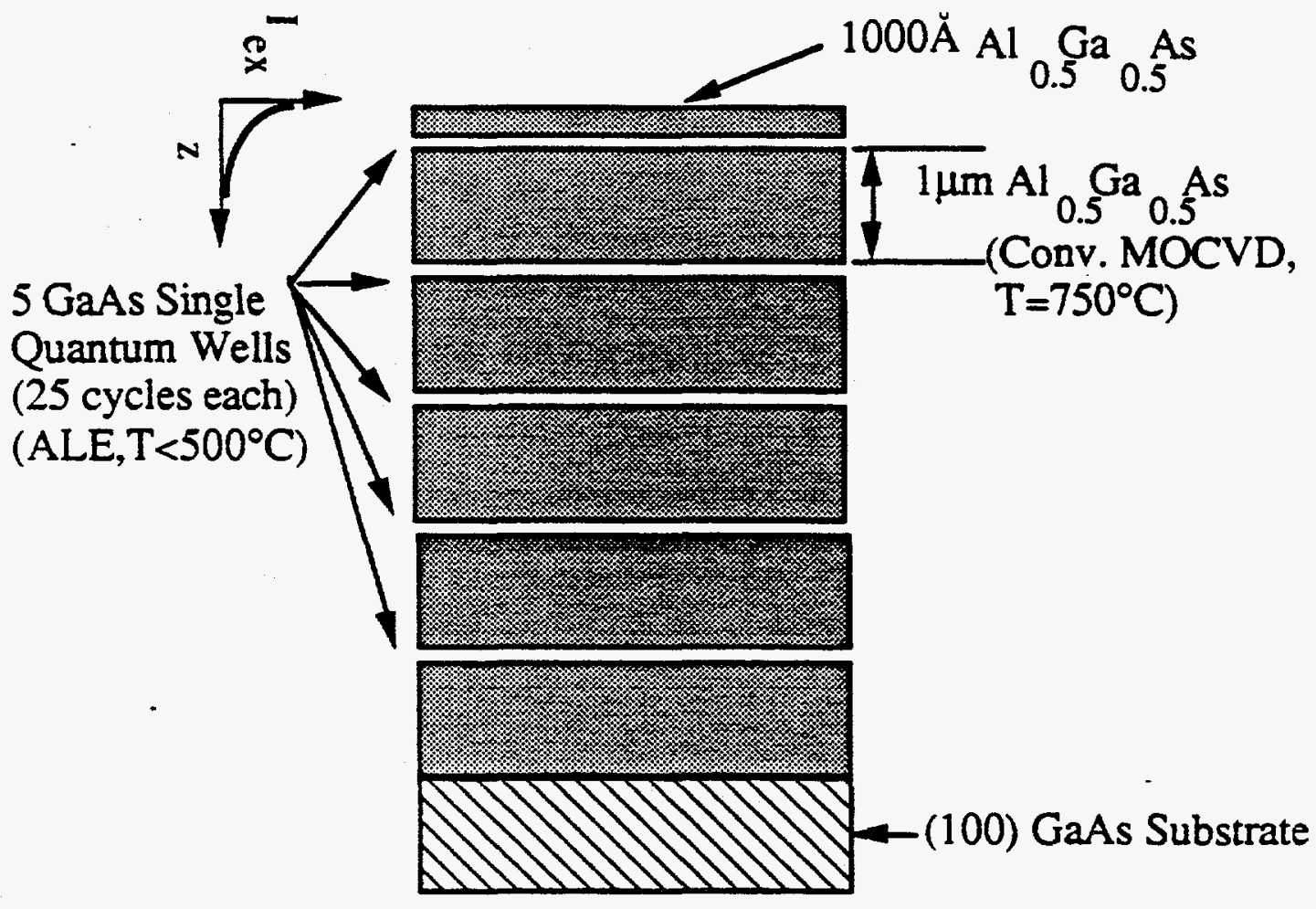

Figure 4.3

Hybrid ALE GaAs/AlGaAs single quantum well sample. Note $1 \mu \mathrm{m}$ thick AlGaAs cladding regions allow separation of PL from the individually grown ALE GaAs quantum wells 
4.4 Growth and Characterization

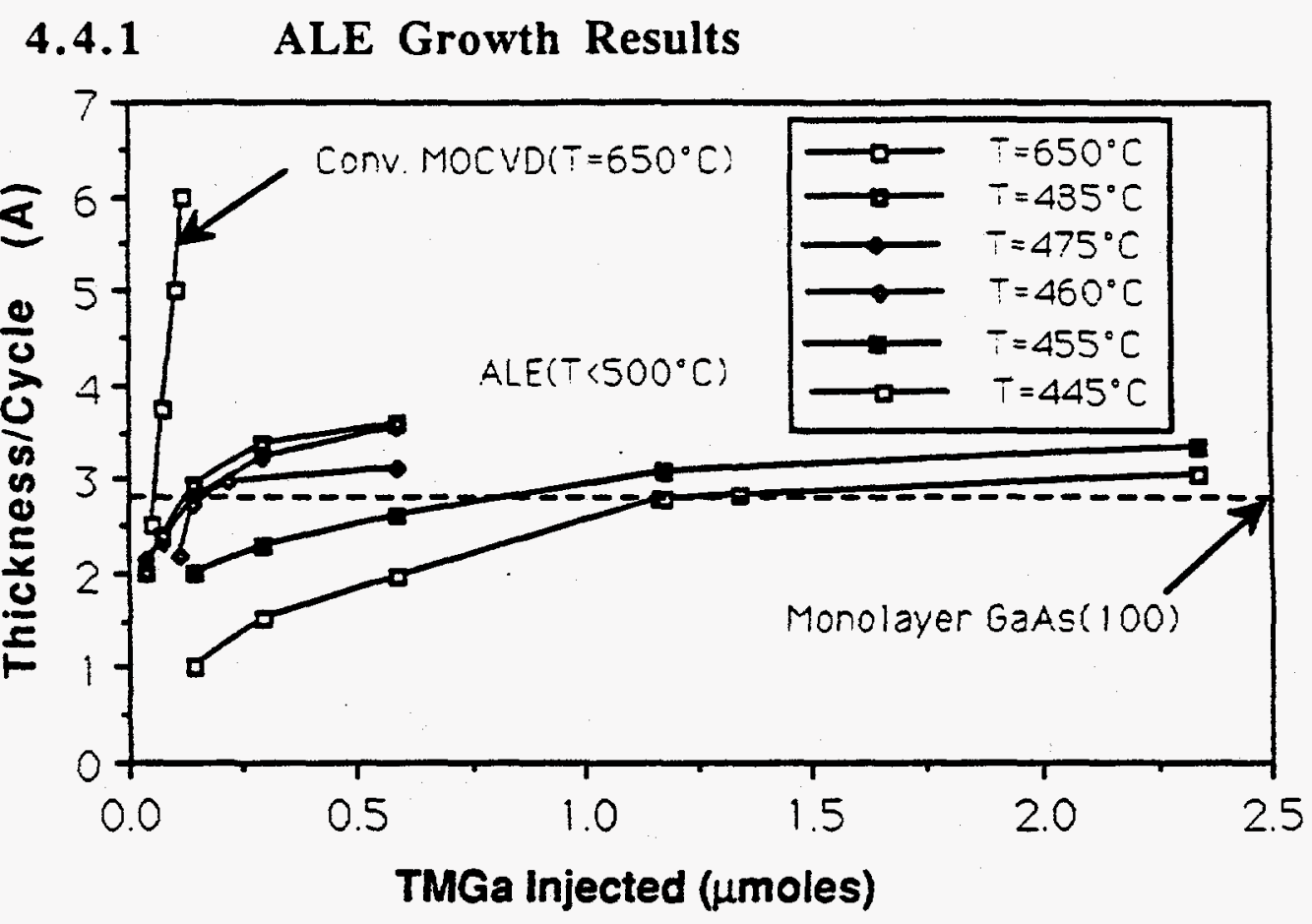

Figure. 4.4.

Comparison of growth rate of GaAs grown utilizing ALE growth regime and conventional MOCVD

Saturated monolayer growth of GaAs has been achieved under conditions where surface reactions dominate the deposition of gallium and arsenic adsorbates. In contrast to conventional MOCVD, in which the growth rate is directly proportional to the TMGa flux in the diffusion controlled regime, the ALE growth rate is strongly sublinear in its dependence on both TMGa and arsine as shown in figures 4.4 and 4.5. The conventional MOCVD data shown in figure 4.4 has been normalized to the same delivery rate as the ALE data to illustrate this point. It is evident that at low temperatures, gas phase reaction rates decrease and 
surface kinetics control the growth rate. Note that the growth rate increases linearly with the reactant volume injected during the cycle up to a critical value near one monolayer and then saturates The chemisorption of a $\mathrm{Ga}$ methyl species is proposed to explain this saturation with TMGa exposure over the temperature range $445^{\circ} \mathrm{C}-485^{\circ} \mathrm{C}$. The fact that the deposited film thicknesses slightly exceed the ideal one monolayer per cycle deposition rate is thought to be caused by the deposition of involatile atomic gallium or incomplete flushing of the reactor. The strong saturation with arsine exposure shown in figure 4.5 indicates that the arsine is not limiting the growth rate and the $\mathrm{Ga}$ deposition step is responsible for the non-ideal saturation of figure 4.4 .

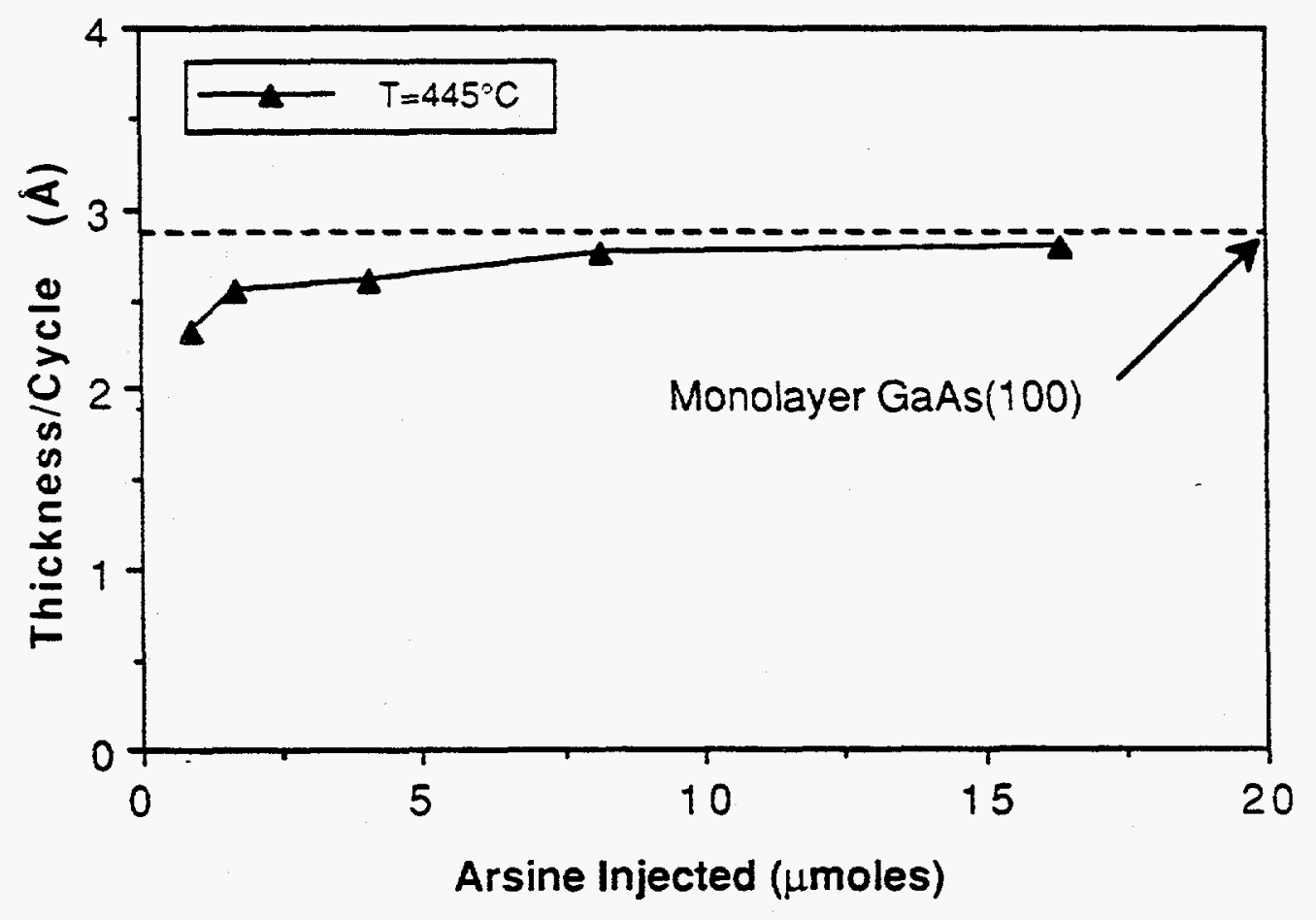

Figure 4.5 .

Growth rate of ALE GaAs layers as a function of arsine injected into reactor. 


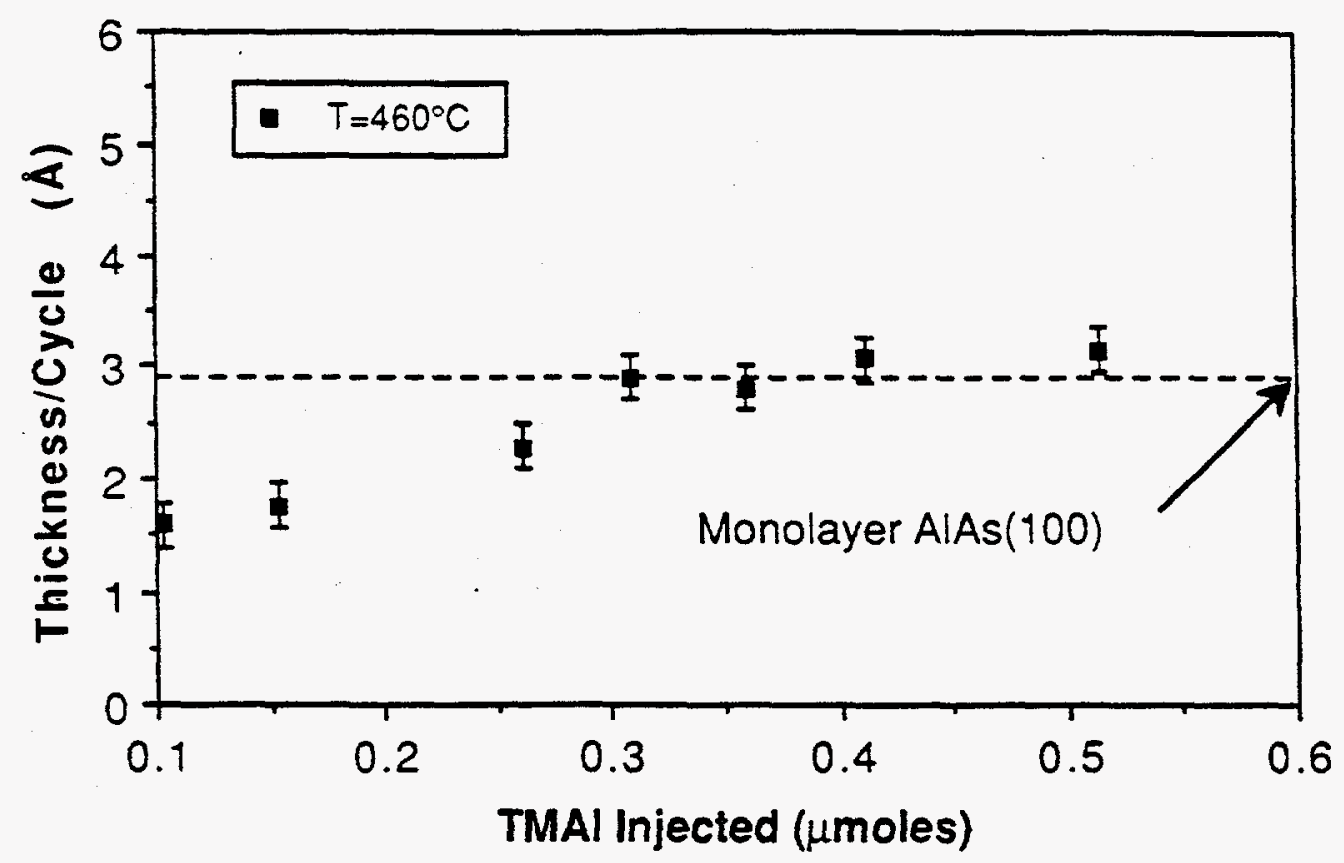

Figure 4.6

Growth rate of AlAs layers grown under ALE conditions as a function of TMAl injected into the reactor.

Deposition of AlAs by ALE also demonstrates a monolayer saturation growth regime as shown by the dependence of growth rate on reactant flux as shown in figure 4.6. Note that the ALE growth rate of AlAs at $460^{\circ} \mathrm{C}$ saturates at slightly higher TMAl quantities than does GaAs using TMGa at the same temperature. We believe that this is most likely due to the lower decomposition rate constant of TMAl. This suggests that partial gas phase or surface decomposition of TMAl also plays an important role in achieving ALE growth. This is supported by the fact that TMAl is a dimer in the gas phase and one would expect that less TMAl 
is needed for saturated growth. ALE of AlGaAs was also achieved by pulsed introduction of the gaseous precursors. Room temperature photoluminescence from both thick AlGaAs ALE layers and quantum wells in which the AlGaAs barrier is also grown by ALE has been studied. The data display the expected behavior, however, low temperature luminescence of ALE AlGaAs exhibits extrinsic luminescence indicating that further optimization of the deposition process is needed for ternary growth.

\subsubsection{Digital Growth Nature}

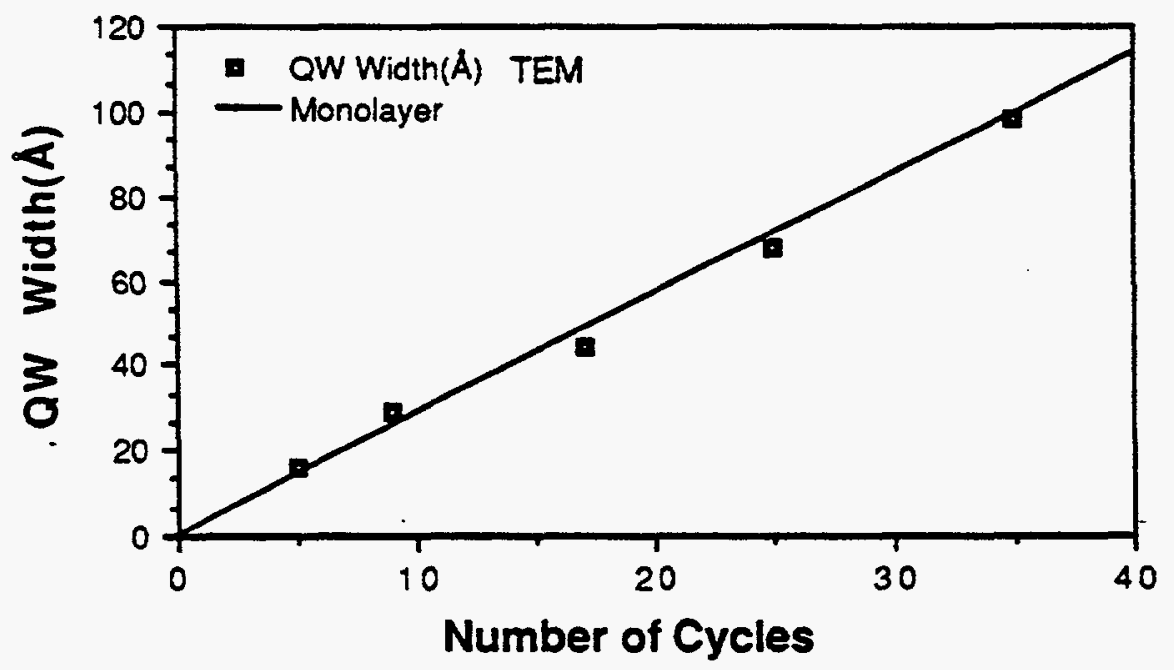

Figure 4.7.

Film thickness dependence on number of ALE deposition cycles demonstrates "digital" nature of ALE growth process.

The "digital" nature of the ALE growth process is best illustrated by the dependence of deposited film thickness on the number of deposition cycles as shown in figure 4.7 where the solid line corresponds to perfect 
monolayer deposition per cycle. Layer thickness measurements for this illustration were performed by cleaved corner TEM which is accurate to within $10 \%$ at the magnification used. Under the conditions identified in figures 4.4 and 4.5 , the grown thickness becomes relatively insensitive to the source gas pressure and the total thickness is determined solely by the number of ALE cycles. Therefore, in comparison with molecular beam epitaxy (MBE) and MOCVD, ALE can be considered a "digital process" since it does not require the precise control of such analog parameters as growth time and reactant flux.

\subsubsection{Thickness Uniformity}

Excellent layer thickness uniformity is one of the inherent advantages expected from using the self-limiting monolayer growth mechanism in the ALE process. We have realized excellent $\mathrm{QW}$ thickness uniformities through the use of the ALE deposition process. Figure 4.8 shows the spatial variation in the ALE GaAs quantum well thickness as a function of distance along the gas flow direction. The QW width variation across the wafer was measured by focusing the excitation laser to a $300 \mu \mathrm{m}$ diameter spot and measuring the peak emission energy shift as function of spot position. A quantum well grown by conventional MOCVD on the same untilted susceptor exhibited a 20-30\% variation in layer thickness due to the gas depletion of TMGa along the gas flow direction. This figure demonstrates the inherent benefits to uniformity that the surface saturated growth mechanism operative in the ALE 
process affords. Therefore, ALE liberates the crystal growth process from the hydrodynamic concerns that plague conventional MOCVD reactor designs. This is of particularly importance in the massproduction of multiple wafers with good uniformity.

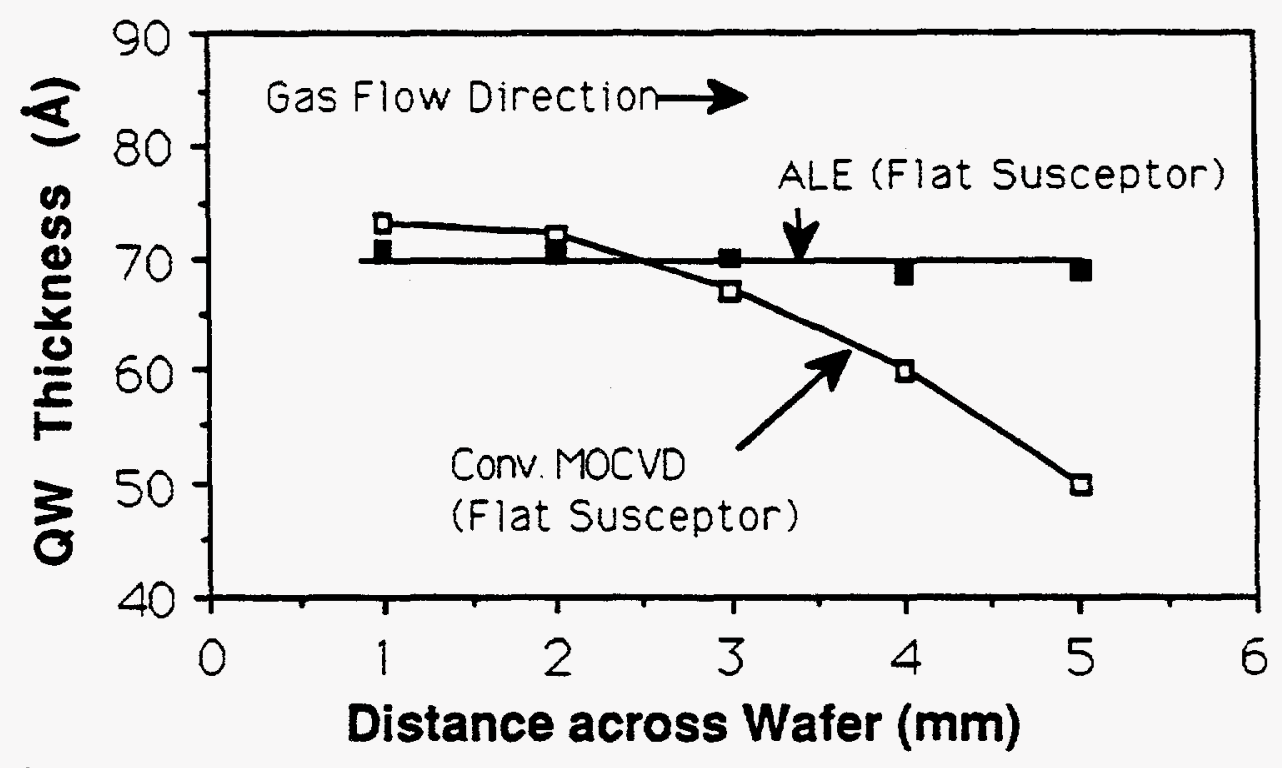

Figure 4.8

QW thickness versus wafer position for quantum wells grown by conventional MOCVD and ALE. 


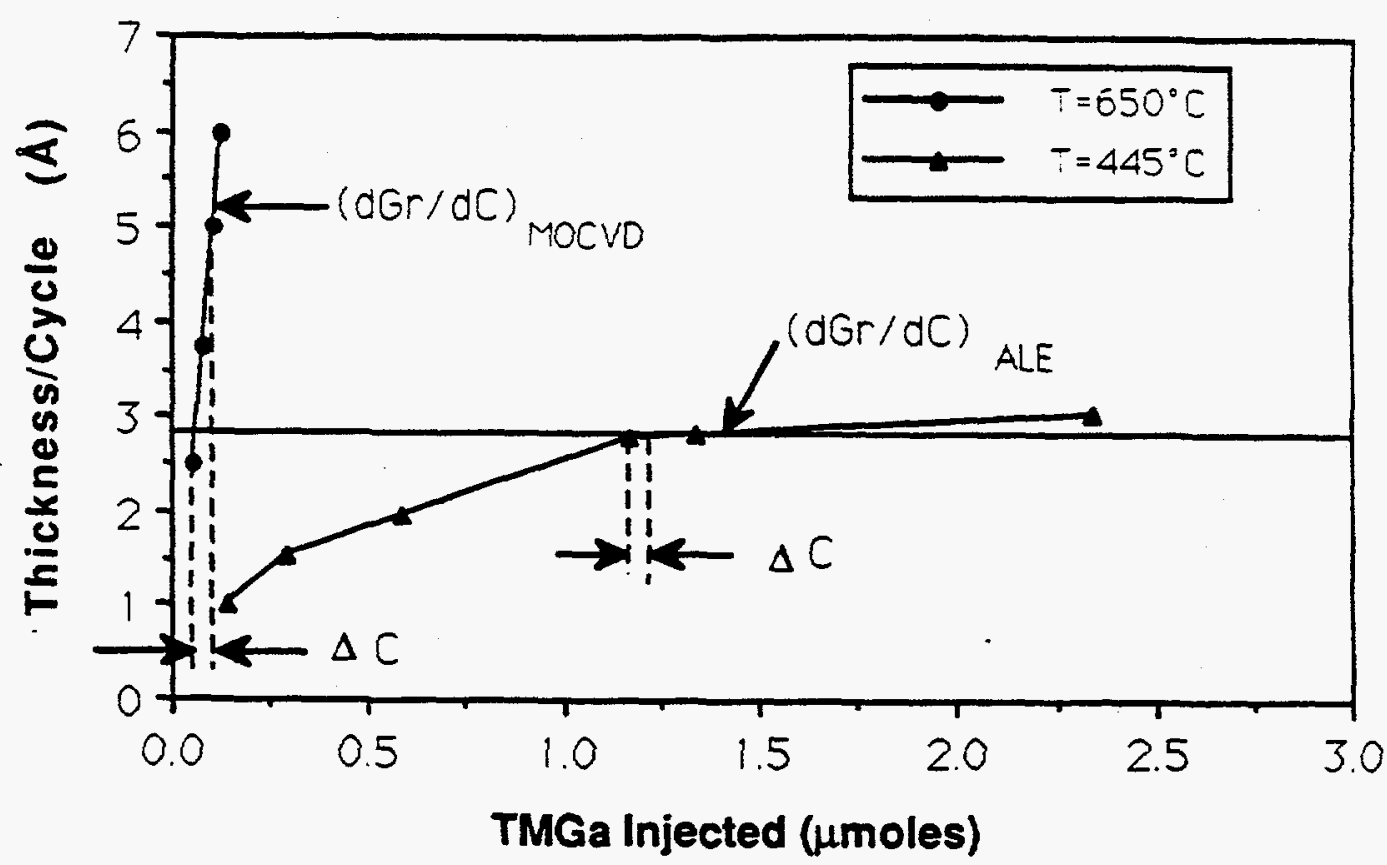

Figure 4.9

The saturated growth mechanism operative in ALE makes the growth rate relatively insensitive to concentration fluctuations.

The excellent thickness uniformities obtainable from ALE, in comparison to conventional MOCVD, can be further explained by analysis of the growth data. As shown in figure 4.9 the slope of the growth rate versus $T M G a$ injected, $\left(\mathrm{dG}_{\mathrm{r}} / \mathrm{dC}_{\mathrm{TMGa}}\right)$, gives a measure of sensitivity of the process to concentration changes. From this graph we can deduce that the ALE process is 230 times less sensitive to concentration fluctuations than conventional MOCVD. For a given gas concentration difference " $\Delta C$ " caused by the gas depletion effect along the gas flow direction we can calculate the corresponding thickness uniformity by the following equation: 


$$
\left(\frac{\Delta T}{T}\right)=\left(\frac{\Delta C\left(\frac{\partial G_{r}}{\partial C}\right)}{G_{r}}\right)
$$

From figure 4.9 we can determine the sensitivity of the growth rate to reactant concentration:

$$
\begin{gathered}
\left(\frac{\partial G_{r}}{\partial C}\right)_{\text {MOCVD }}=\frac{57.5 \mathrm{~A}}{\mu \text { moles }} \\
\left(\frac{\partial G_{r}}{\partial C}\right)_{A L E}=\frac{0.25 \mathrm{~A}}{\mu \text { moles }}
\end{gathered}
$$

For a gas concentration depletion of just $0.02 \mu$ moles this results in $32.9 \%$ thickness change across the wafer for conventional MOCVD. In contrast, for the same concentration fluctuation ALE will theoretically yield excellent uniformity control with a thickness variation of just $0.14 \%$ ! 


\subsubsection{Cleaved Corner TEM}

Epitaxial layer thickness measurements of the GaAs quantum wells are made by transmission electron microscopy (TEM) analysis of the freshly cleaved single crystal corner and photoluminescence emission energy from the individual wells. Utilizing the natural (110) cleavage planes in GaAs, it is possible to obtain an extremely sharp corner, the thinnest portion of which can be penetrated by the electrons. Thus by orienting the edge of the sample at $45^{\circ}$ with respect to the electron beam, a thin area exists near the corner allowing TEM analysis of layers in the cross section. Figure 4.10 illustrates the geometry of the TEM imaging conditions. This is relatively new technique, ${ }^{1}$ which eliminates the time consuming sample preparation usually required for TEM evaluation of semiconductor layer thickness measurements. Evaluation by corner TEM and photoluminescence results confirm that the ALE growth process is controlled at the atomic level. Analysis of ALE AlAs films was performed by cleaved corner TEM, SEM and angle lapping measurements of thicker layers. 


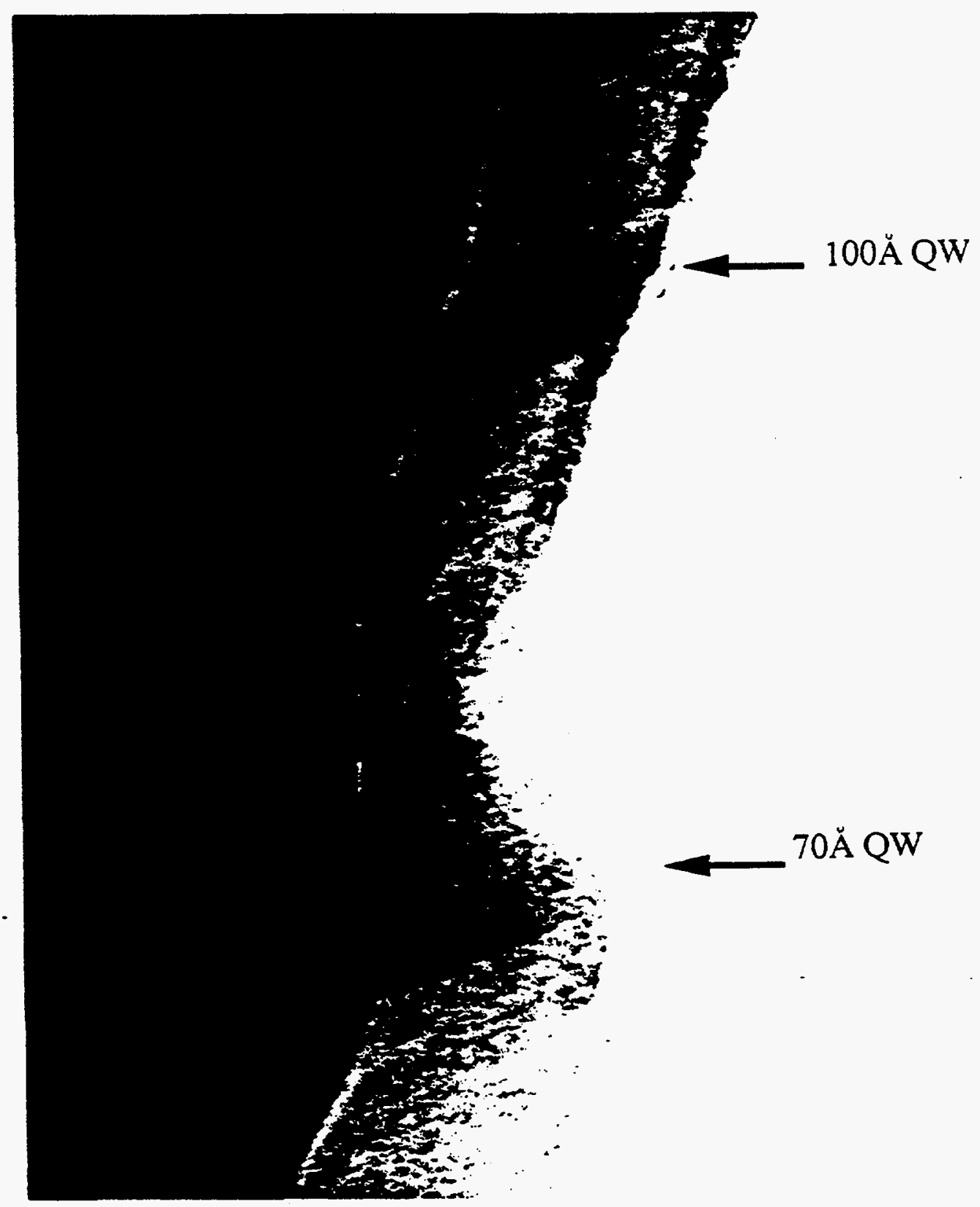

Figure 4.10

Cleaved-corner transmission electron micrograph of two ALE grown $\mathrm{GaAs}$ quantum wells (MAG $=1,200 \mathrm{kX}$ ) 


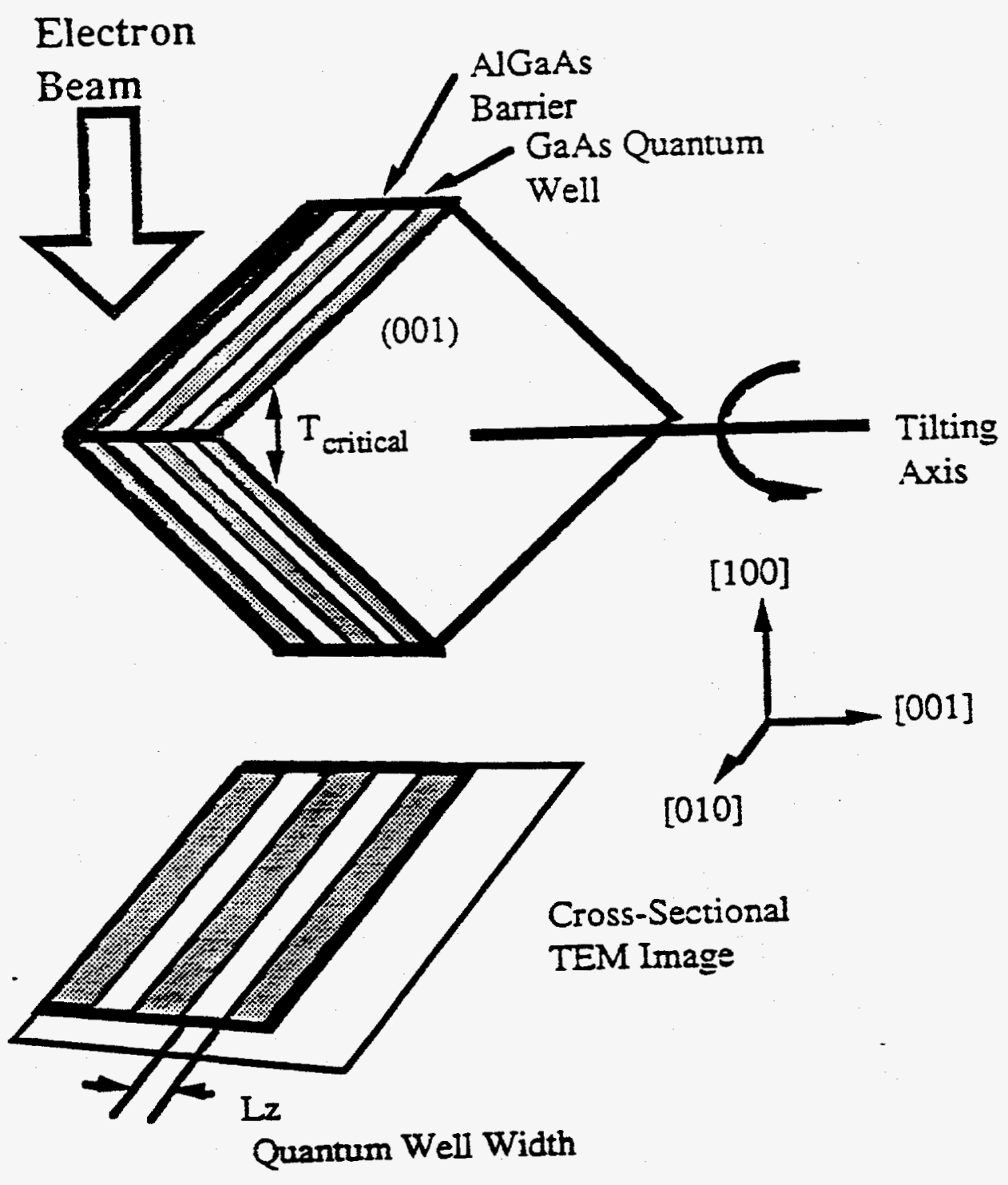

Figure 4.11

Schematic illustration of geometry of imaging conditions utilized in cleaved-corner TEM evaluation of GaAs/AlGaAs sample (modified from Kakibayashi et al.). 2 


\subsubsection{SEM Evaluation of ALE layer thickness}

Scanning Electron Microscopy has been used to verify the monolayer deposition nature of the ALE process. Accurate SEM measurements can be made by growing thick ALE GaAs and AlAs layers 1000-1500 $\AA$ thick, and confining them between AlGaAs or GaAs for contrast. Figure 4.12 shows a typical structure consisting of three ALE AlAs with GaAs cladding regions, in which the ALE layers are grown under varying TMAl fluxes. In this structure, each layer was grown at $460^{\circ} \mathrm{C}$ with twice the TMAl flux of the layer preceding it. For fluxes above the critical amount, the layer thickness saturate at the expected monolayer deposited thickness of $1500 \AA$ for 530 cycles

\subsubsection{Morphology ALE GaAs}

The surface morphology of GaAs grown by ALE is smooth with mirror-like surfaces. Figure 4.13 shows the surface of a 1 micron GaAs sample grown by ALE at $460^{\circ} \mathrm{C}$. The defect on the left side is typical of our conventional grown GaAs and is thought to be cause by a defect or particulate matter at the interface between the grown layer and susceptor. The smoothness of the surface surrounding the defect provide evidence that $\mathrm{Ga}$ is not balling up on the surface. If the growth temperature is too high, we violate the conditions under which the surface saturation growth mechanism dominates; therefore, deposition of involatile gallium occurs and we observe dendritic growth. Figure 4.14 shows an SEM of dendritic growth occurring on a sample grown by ALE at $540^{\circ} \mathrm{C}$ 

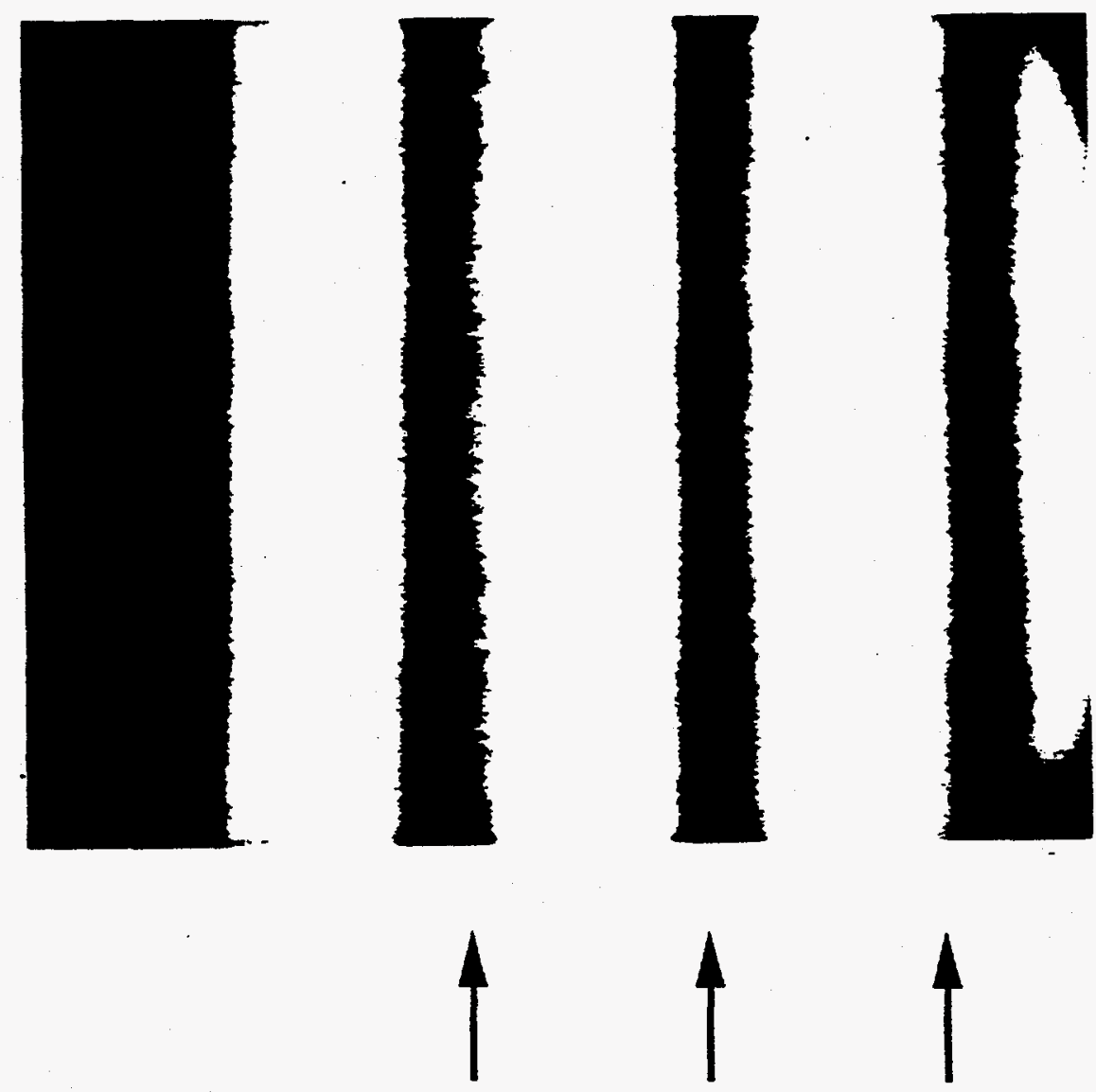

Figure 4.12

SEM micrograph of 3 ALE AlAs layers with GaAs cladding regions. Each layer is grown with twice the TMAl flux of the layer preceding it. $(\mathrm{MAG}=56 \mathrm{kX})$ 
Figure 4.13

Smooth surface morphology of $1 \mu \mathrm{m}$ thick GaAs grown by ALE at $460^{\circ} \mathrm{C}$. 


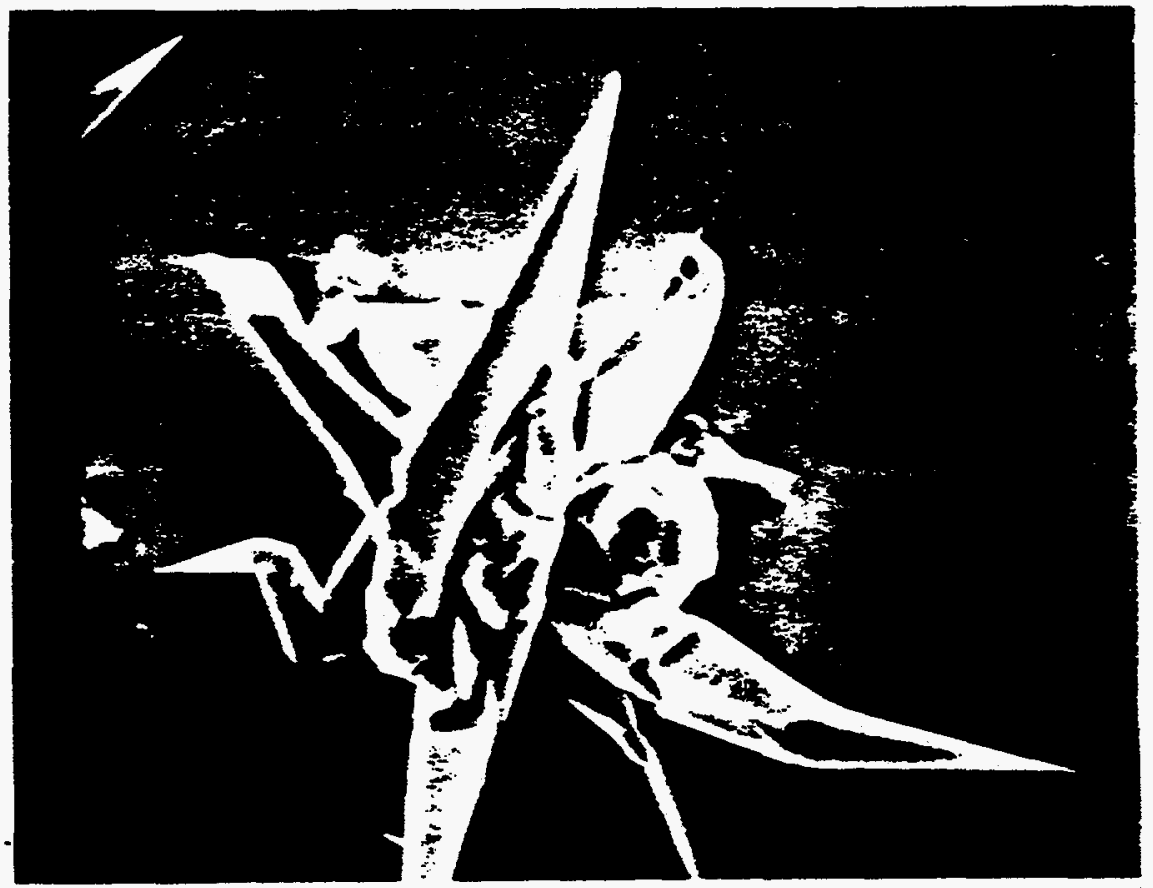

Figure 4.14

Surface morphology of ALE layers grown above $500^{\circ} \mathrm{C}$ exhibit dendritic growth indicative of excess $\mathrm{Ga}$ (SEM $5 \mathrm{kX}$ ). 


\subsubsection{Electrical Characterization}

In order to perform electrical characterization of the epitaxial layers at least $1 \mu \mathrm{m}$ of material is needed to compensate for the depletion depth created by the electric field from the ohmic contacts. Because of the slow growth rate in $\operatorname{ALE}(0.71 \AA / \mathrm{sec})$, only a few thick samples were grown for electrical characterization. These samples required 3533 ALE cycles and took approximately 4 hours to grow. Hall measurements at room temperature and $77 \mathrm{~K}$ were performed on $1 \mu \mathrm{m}$ thick ALE GaAs layers using the Van der Pauw technique. ${ }^{2}$ Ohmic contact was made to these samples by alloying Indium-Zinc ( $99 \% \mathrm{In}-1 \% \mathrm{Zn}$ ) dots. As listed in Table 4.1, all ALE layers exhibit high p-type background carrier concentrations with low mobilities. This high p-type background doping has been observed in all the previous studies of ALE GaAs.3,4 Methyl radicals adsorbed on As sites are thought to lead to p-type doping in MOCVD. 5 Therefore, incompletion of the surface reaction between the gallium 'methyl adsorbate and arsine would lead to the p-type doping observed in ALE films.

Recently Colas et al. 6 have obtained significantly lower background doping, n-type $3 \times 10^{15} \mathrm{~cm}^{-3}$, and higher mobility values of $35,000 \mathrm{~cm}^{2} / \mathrm{V} \cdot \mathrm{s}$ at $77 \mathrm{~K}$. The highest mobility samples were found to be grown by using $1 / 2$ monolayer surface coverage of the column III source and no purge after the arsine exposure step. They explain their results by noting that carbon incorporation depends on the effective V/III ratio at the surface. In conventional MOCVD it is well established that an 
increase in the V/III ratio results in a decrease in carbon incorporation. ${ }^{7}$ Therefore, if no purge follows the arsine step there will be minimal arsine desorption and the V/III ratio is at its peak. These results indicate that with further optimization, the ALE process could possibly grow high purity GaAs. Rather than focussing on electrical properties of ALE, we have elected to optimize ALE for the growth of ultrathin quantum well layers and incorporate these layers in devices. As we will demonstrate in the next two chapters high quality quantum wells and heterostructure devices can be grown by ALE.

Table 4.1 Electrical characteristics of ALE GaAs.

\begin{tabular}{|cll|}
\hline Growth Temperature & Carrier Conc. & \multicolumn{1}{c|}{ Mobility } \\
\hline $460^{\circ} \mathrm{C}$ & $1 \times 10^{17} \mathrm{~cm}^{-3}$ & $240 \mathrm{~cm}^{2} / \mathrm{V} \cdot \mathrm{s}(300 \mathrm{~K})$ \\
\hline & & $940 \mathrm{~cm}^{2} / \mathrm{V} \cdot \mathrm{s}(77 \mathrm{~K})$ \\
\hline $500^{\circ} \mathrm{C}$ & $3 \times 10^{18} \mathrm{~cm}^{-3}$ & $110 \mathrm{~cm}^{2} / \mathrm{V} \cdot \mathrm{s}(300 \mathrm{~K})$ \\
\hline & & $480 \mathrm{~cm}^{2} / \mathrm{V} \cdot \mathrm{s}(77 \mathrm{~K})$ \\
\hline
\end{tabular}




\subsubsection{Optical Properties}

Strong intense photoluminescence (PL) is observed from the ALE GaAs layers at both room temperature and 4.8K. A cw Argon ion laser $(\lambda=514 \mathrm{~nm})$ was used as the excitation source with power densities in the $10-1000 \mathrm{~mW} / \mathrm{cm}^{2}$ range. As shown in figure 4.15 , low temperature PL spectra exhibits an intense free exciton (FX) peak at $8180 \AA$ and impurity related luminescence which can be attributed to carbon. The luminescence intensity observed from ALE GaAs films is approximately equal to that from conventional grown GaAs films at equal excitation powers. This demonstrates that ALE GaAs has good optical efficiency and is not dominated by non-radiative recombination. Therefore, optical devices incorporating ALE layers should exhibit good characteristics. 


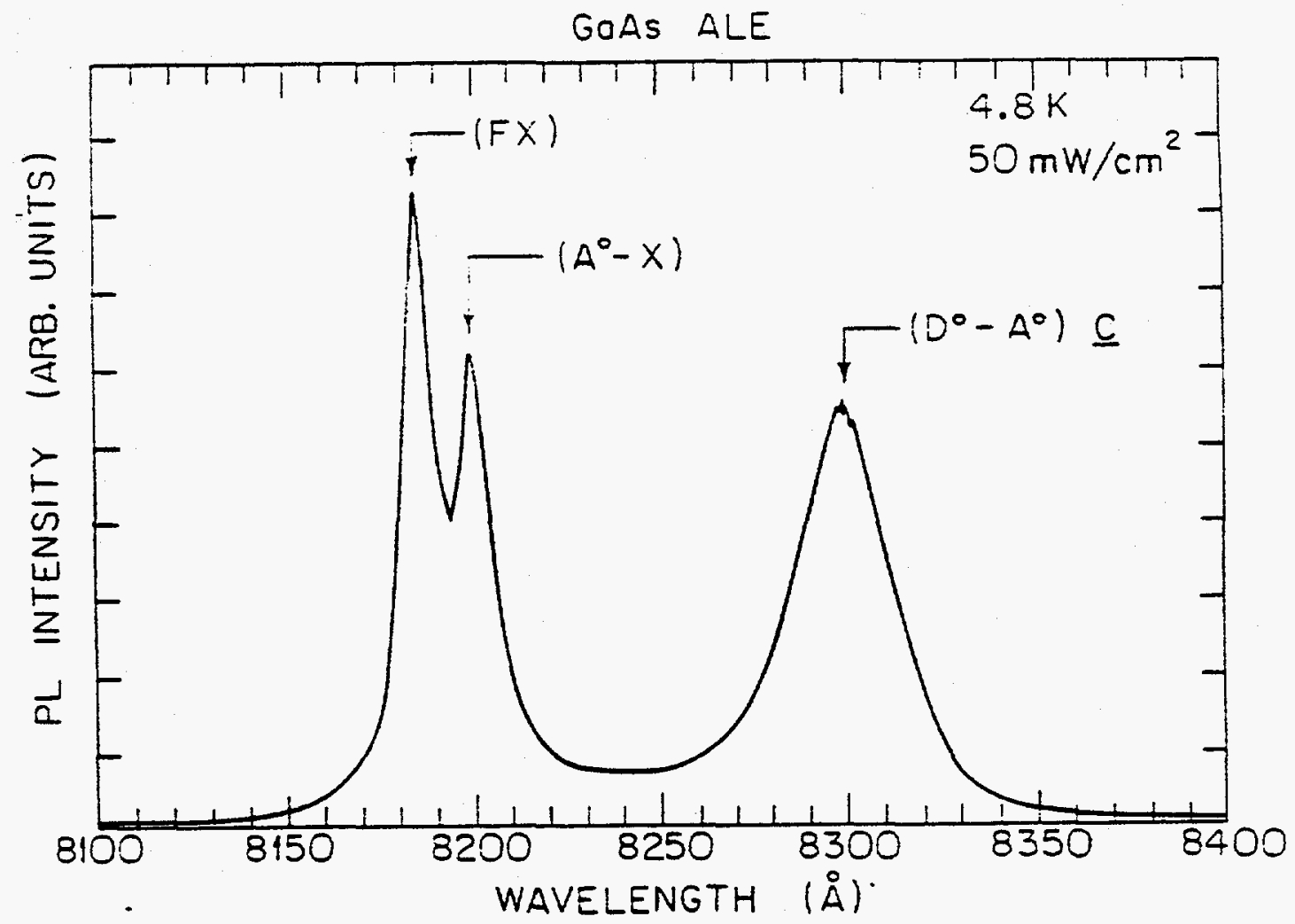

Figure 4.15

Low temperature photoluminescence of ALE GaAs exhibits intense free exciton peak and donor to acceptor (carbon) peak. 


\subsection{ALE GROWTH MODEL}

\subsubsection{Proposed ALE Reaction Model}

We propose that the self-limiting mechanism operative in the ALE process consists of the partial decomposition of trimethylgallium followed by the chemisorption of the gallium adsorbate on arsenic sites. The most plausible series of reactions occurring in the ALE process are as follows:

$$
\begin{gathered}
\mathrm{Ga}\left(\mathrm{CH}_{3}\right)_{3(\mathrm{v})} \rightarrow \mathrm{Ga}\left(\mathrm{CH}_{3}\right)_{2}+\mathrm{CH}_{3}(\mathrm{v}) \\
\mathrm{Ga}\left(\mathrm{CH}_{3}\right)_{2(\mathrm{v})} \rightarrow \dot{\mathrm{Ga}}\left(\mathrm{CH}_{3}\right)+\mathrm{CH}_{3}(\mathrm{v}) \\
\mathrm{Ga}\left(\mathrm{CH}_{3}\right)_{\text {(chem. ads.)As site }}+\mathrm{AsH}_{3(\mathrm{~s} / \mathrm{v})} \rightarrow \mathrm{GaAs}_{(\mathrm{s})}+\mathrm{CH}_{4(\mathrm{v})}+\mathrm{H}_{2(\mathrm{v})}
\end{gathered}
$$

This view is supported by our experimental observations along with the recent research of several other groups. Upon injecting arsine onto a gallium adsorbate saturated surface, Nishizawa et al. ${ }^{8}$ observe the release of methane into the vapor phase. In another study, ${ }^{9}$ ALE growth is observed to occur on the arsenic terminated (111)B plane, but no growth was observed on the gallium terminated (111)A plane. Therefore, the gallium methyl complex may adsorb on arsenic atoms but it does not adsorb on $\mathrm{Ga}$ atoms at the surface. In Chapter 3, data was presented which indicated that TMGa decomposes in two stages. In the first stage, which occurred in the temperature range $380^{\circ} \mathrm{C}-440^{\circ} \mathrm{C}$ in the decomposition experiments, TMGa sequentially loses two methyl radicals in rapid succession. The third methyl radical is lost only in the second 
stage at higher temperatures to release atomic Ga. Thus it is likely that the partial gas or surface phase decomposition of $\mathrm{TMGa}$ in $\mathrm{H}_{2}$ at the temperatures and times employed here $(1 \mathrm{sec})$ for ALE will result in the formation of $\mathrm{CH}_{3} \mathrm{Ga}$. The saturated monolayer growth we have observed suggests that the adsorbed species must retain some of the organic radicals to remain volatile enough to allow only one monolayer to be chemisorbed and avoid the deposition of excess $\mathrm{Ga}$. This is confirmed by the fact that other researchers observe the formation of $\mathrm{Ga}$ droplets at higher temperatures. 10 Therefore we conclude that $\mathrm{CH}_{3} \mathrm{Ga}$ is the surface adsorbed species in our experiments. Figure 4.16 illustrates the surface reactions which we believe lead to the self-limiting growth observed in the ALE process. 

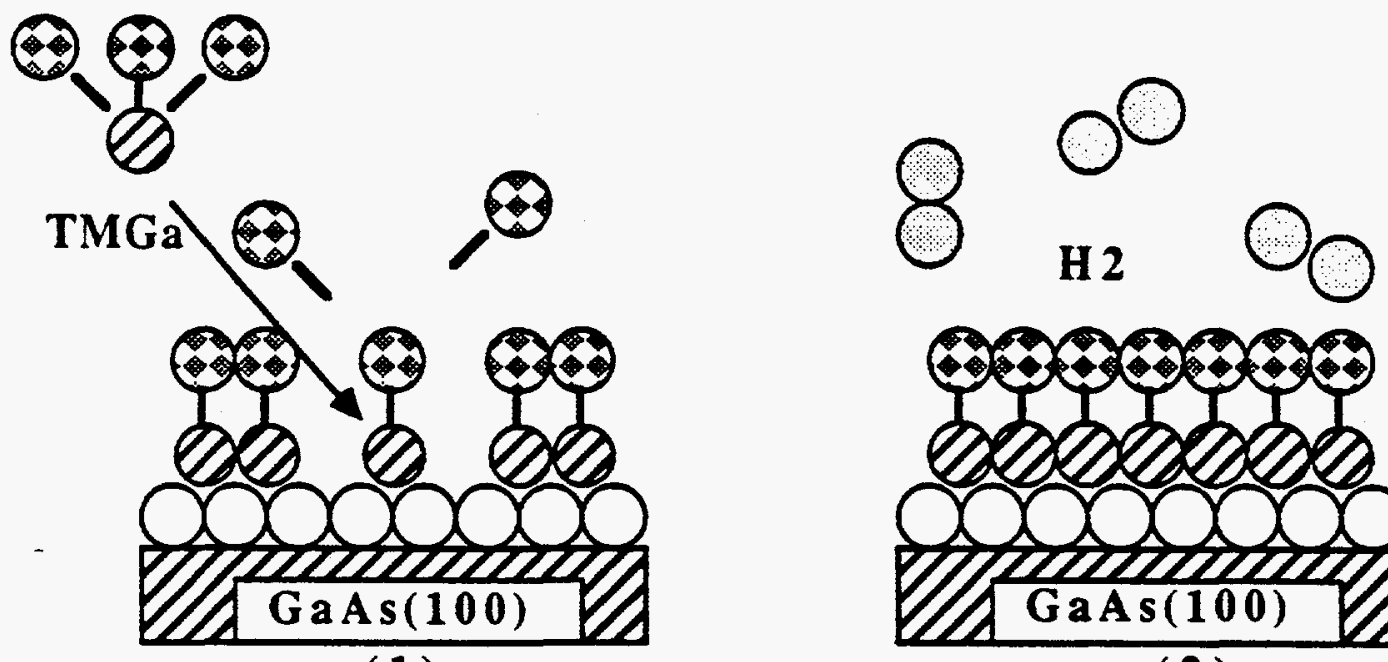

(1)

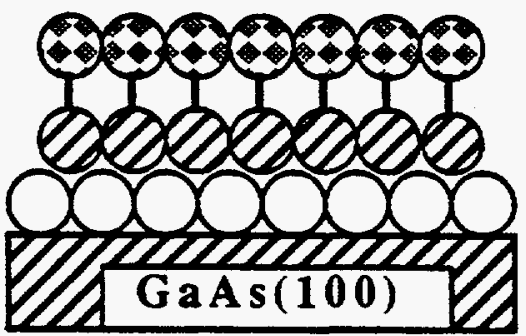

(2)

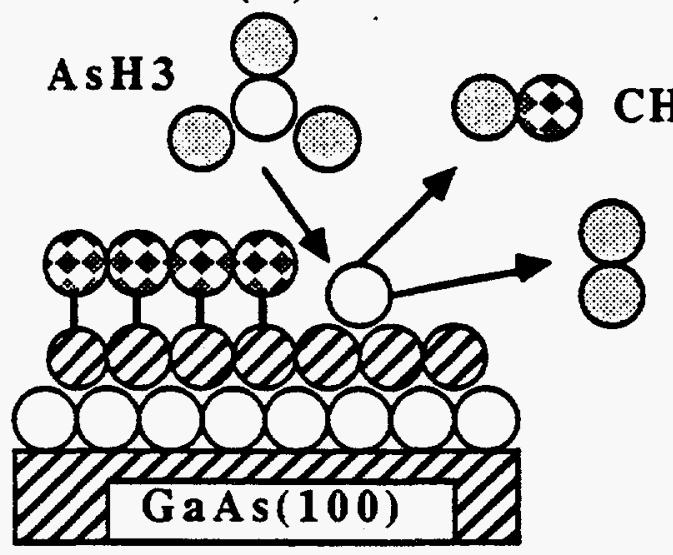

(3)

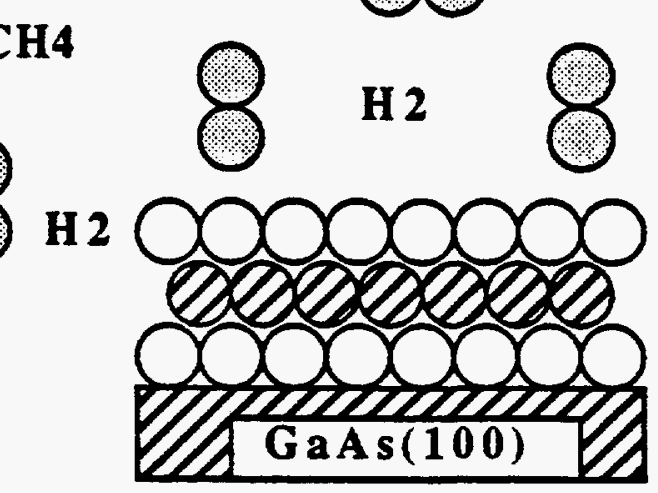

(4)

Figure 4.16

Schematic illustration of the growth model showing Galliummethyl radicals chemisorbed on the surface. Layer growth is completed by the surface reaction of $\mathrm{AsH}_{3}$ with the gallium adsorbate.

\subsubsection{Calculated Surface Coverages}

To achieve atomic layer epitaxy the deposited number of atoms should be approximately equal to the surface density in the plane of interest. The surface density of atoms in the (100) plane of GaAs is 
$6.26 \times 10^{14}$ atoms $/ \mathrm{cm}^{2}$. Using Fick's equation for flux through the boundary layer, equation 2.7 Chapter 2 , we can get a measure of the excess surface coverage of TMGa on the GaAs surface The amount of surface coverage " $\Theta$ " can then be estimated by divided the flux of molecules to the surface by the surface site density. As shown in Table 4.2 , the surface densities of TMGa provided for observed perfect monolayer coverages $(\Theta=1)$, are in excess of the available surface sites at all the temperatures over which saturated growth was observed. Therefore, it appears that a temperature activated process is necessary to drive the chemisorption of a gallium adsorbate.

\section{Table 4.2 Excess Surface Coverages( $(\theta)$}

Temp. TMGa Injected TMGa Surface Coverage

\begin{tabular}{|llll|}
$\left({ }^{\circ} \mathrm{C}\right)$ & for $\Theta=1$ & Flux & $\Theta$ \\
\hline 485 & $0.13 \mu$ moles & $5.38 \times 10^{16}$ molecules $/ \mathrm{cm}^{2} \mathrm{sec}$ & 1.3 \\
\hline 460 & $0.26 \mu$ moles & $1.08 \times 10^{17}$ molecules $/ \mathrm{cm}^{2} \mathrm{sec}$ & 2.6 \\
\hline 445 & $1.3 \mu$ moles & $5.40 \times 10^{17}$ molecules $/ \mathrm{cm}^{2} \mathrm{sec}$ & 13 \\
\hline
\end{tabular}

Further evidence of a temperature driven adsorption process is the fact that the actual number of collision of the TMGa molecule with each surface site yields an even greater value of excess surface saturation. The impinging collision rate " $R_{i}$ " of molecules on the surface can be determined from the average speed of the molecules in the gas phase and the number density of TMGa. If we assume that the thermal energy of the gas is equal to the total kinetic energy of all moving particles, then the 
impinging rate of molecules on a planer surface at any given temperature $T_{\mathrm{m}}$ can be expressed as: 11

$$
R_{i}=\frac{N_{g}}{4} \sqrt{\frac{8 k T_{m}}{\pi M}}
$$

where: $\mathrm{Ng}$ is the gas phase density of column III molecules $/ \mathrm{cm}^{3}, \mathrm{M}$ is the molecular weight of the column III source, $\mathrm{T}_{\mathrm{m}}$ the mean reactor temperature. The amount of excess collisions per site "\# $\Theta$ " is then given by the impinging rate $R_{i}$ time the exposure time " $\tau$ ", divided by the surface site density $\mathrm{N}_{\mathrm{S}}$.

$$
\# \theta=\frac{R_{i} \tau}{N_{s}}
$$

For the partial pressures of TMGa utilized in our study the number of excess collisions for each surface site is from $800-8000$. Thus it is obvious that the ALE process is not limited by simply the supply of available reactant, but rather by kinetics of the surface adsorption process. 


\subsubsection{Kinetic Models of ALE Process}

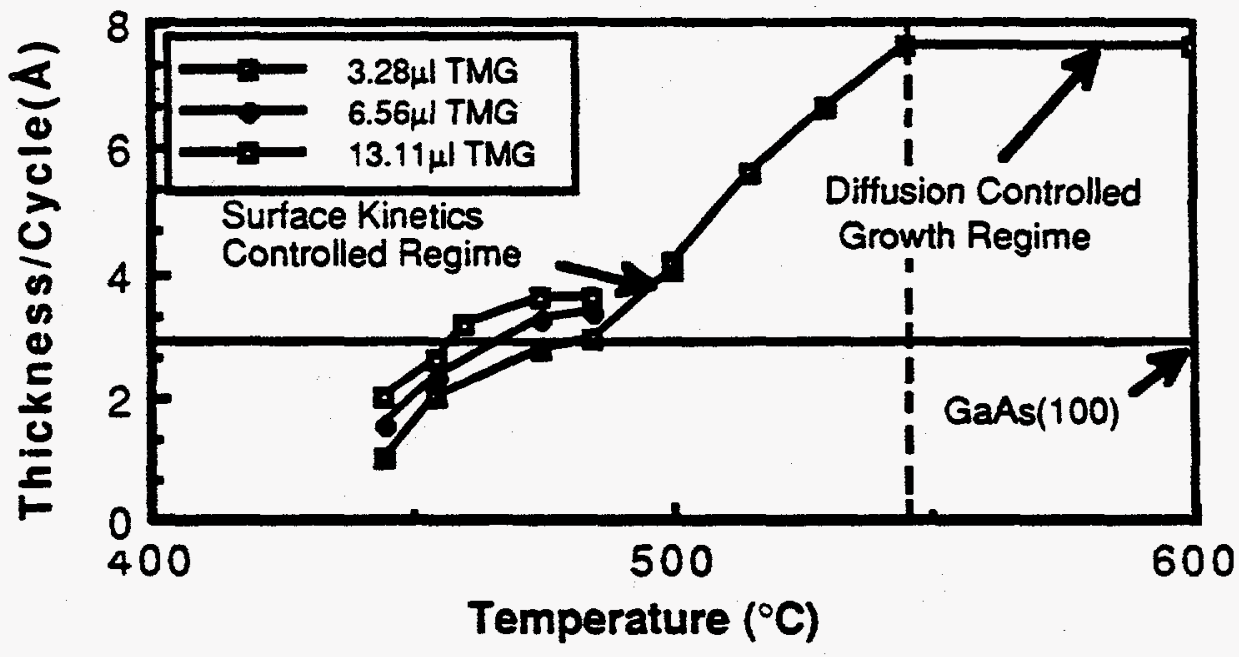

Figure 4.17

Effect of temperature on growth rate in surface reaction controlled ALE growth regime.

\subsubsection{ALE Growth Model 1}

The observation of increasing surface coverage with increasing temperatures is contrary to normal adsorption models. When the species is physisorbed on the surface, the temperature dependence of $\Theta$ is controlled by the rate of desorption, $k d e s$, and $d \Theta / d t$ decreases with increasing temperature. The data of figure 4.17 show the opposite trend. This indicates that the adsorbed species are chemisorbed and that rate of adsorption, kads, controls the temperature dependence of $\Theta$.This trend is 
illustrated in figure 4.17. Therefore, we expect that $\mathrm{k}_{\mathrm{ads}}$ will be dependent upon some gas phase or surface reaction that is more strongly activated than the surface desorption process.

We propose that a two process model occurs in thermally driven atomic layer epitaxy. The first step is the thermal decomposition of TMGa which generates gallium methyl radicals. The second step is the adsorption of the reactive species, which saturates with increasing partial pressures because of the limited adsorption sites. We assume that the diffusive flux of the adsorbate is proportional to the reactant concentration in the gas phase, $C_{T M G a}$ (atoms $/ \mathrm{cm}^{3}$ ), proportional to the gas phase or surface reaction rate that produces the adsorbate, $\mathrm{kg}\left(\mathrm{sec}^{-1}\right)$, and proportional to the diffusion distance which we take to be the boundary layer, $\delta(\mathrm{cm})$. The effective adsorption is given by

$$
\mathrm{k}_{\mathrm{ads}}=\frac{\delta \mathrm{k}_{\mathrm{TMGa}} \mathrm{C}_{\mathrm{TMGa}}}{\mathrm{N}_{\mathrm{s}}}
$$

where $\mathrm{N}_{S}$ is the atomic surface site density in $\mathrm{cm}^{-2}$.

The saturated ALE growth data from figures 4.4-4.6, along with the observed temperature dependence, can be qualitatively explained by a first order adsorption model which takes into account the temperature activated generation of a gallium adsorbate. We describe the rate of 
surface coverage $(d \Theta / d t)$ as the difference between a surface adsorption rate, $\mathrm{k}_{\mathrm{ads}}$, and a surface desorption rate, $\mathrm{k}_{\mathrm{des}}$.

$$
\frac{d \theta}{d t}=k_{\text {ads }}(1-\theta)-k_{\text {des }}(\theta)
$$

Integration leads to a simple Langmuir adsorption isotherm for the surface coverage.

$$
\theta=\left(\frac{\mathrm{k}_{\mathrm{ads}}}{\mathrm{k}_{\mathrm{ads}}+\mathrm{k}_{\mathrm{des}}}\right)\left[1-\exp \left[-\left(\mathrm{k}_{\mathrm{ads}}+\mathrm{k}_{\mathrm{des}}\right) \mathrm{t}\right]\right]
$$

where both $\mathrm{k}_{\mathrm{ads}}$ and $\mathrm{kdes}$ are functions of temperature. 


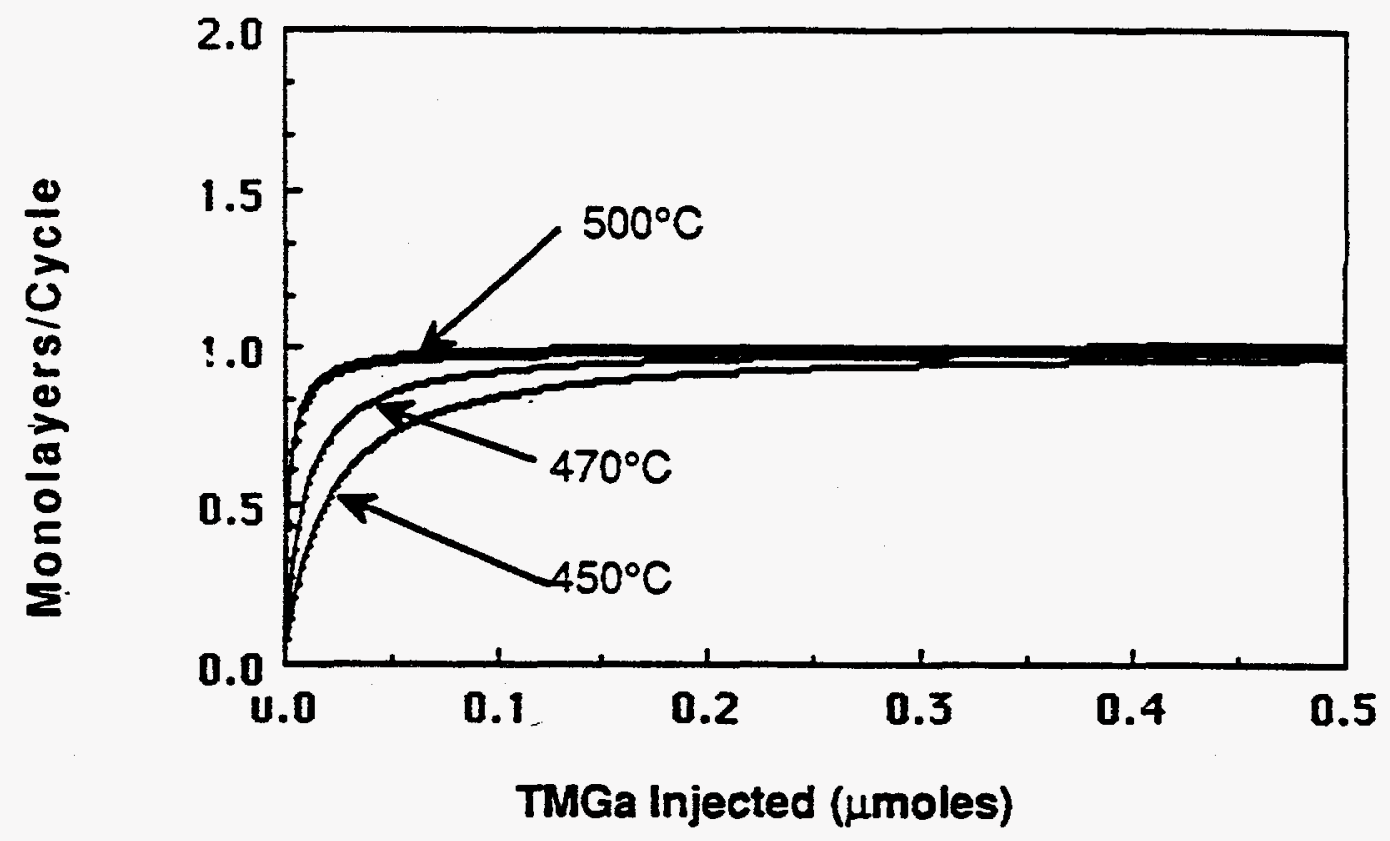

Figure 4.18

First order model predicts self-limiting growth in the same temperature range as observed experimentally.

Figure 4.18 shows the computer generated curves for the first order adsorption model. Using the measured rate constant for removal of the first methyl group from TMGa presented in Chapter 3, we observe that the model predicts self-limiting growth in the same time and temperature range as was observed experimentally. However, a more accurate model should take into account the non-ideal saturation observed experimentally. 


\subsubsection{ALE Growth Model 2}

To account for the observed deviation from perfect monolayer growth we introduce a rate expression $(\mathrm{kGa})$ which predicts the amount of involatile $\mathrm{Ga}$ produced by the pyrolysis of monomethylgallium. The rate of deposited $\mathrm{Ga}$ will be independent of surface coverage since it has a low vapor pressure and can not evaporate as readily as the gallium methyl complex. The first order adsorption model is then corrected to the following expression:

$$
\frac{d \theta}{d t}=k_{a d s}(1-\theta)-k_{\text {des }}(\theta)+k_{G a}
$$

Where:

$$
\mathrm{k}_{\mathrm{Ga}}=\frac{\left(\delta \mathrm{k}_{\mathrm{MM}} \mathrm{C}_{\mathrm{MM}}\right)}{\mathrm{N}_{\mathrm{s}}}
$$

$\mathrm{k}_{\mathrm{Ga}}$ utilizes the activation energy $(-77.5 \mathrm{kcal} / \mathrm{mole})$ required for the removal of the third methyl radical from TMGa which determined by Jacko and Price. 12 The frequency factor" $A$ " for the rate constant $k_{M M}$ in hydrogen was estimated by setting the relative concentration of the third methyl group to $99 \%$ at $460^{\circ} \mathrm{C}$, as was observed in figure 3.9 . Integration of equation 4.11 leads to the following adsorption isotherm for the surface coverage (See Appendix A for Integration).

$$
\theta=\left(\frac{\mathrm{k}_{\mathrm{Ga}}+\mathrm{k}_{\text {ads }}}{\mathrm{k}_{\mathrm{ads}}+\mathrm{k}_{\text {des }}}\right)\left[1-\exp \left[-\left(\mathrm{k}_{\mathrm{ads}}+\mathrm{k}_{\mathrm{des}}\right) \mathrm{t}\right]\right]
$$


The concentration of monomethylgallium $\left(\mathrm{C}_{\mathrm{MM}}\right)$ can be estimated from an earlier kinetic study in which removal of the first methyl from TMGa was observed to be the rate limiting step.

$\mathrm{CMM}_{\mathrm{MM}}=\mathrm{Ng}\left\{1-\exp \left[-\mathrm{kTMGa}_{\mathrm{TM}} \tau\right]\right\}$

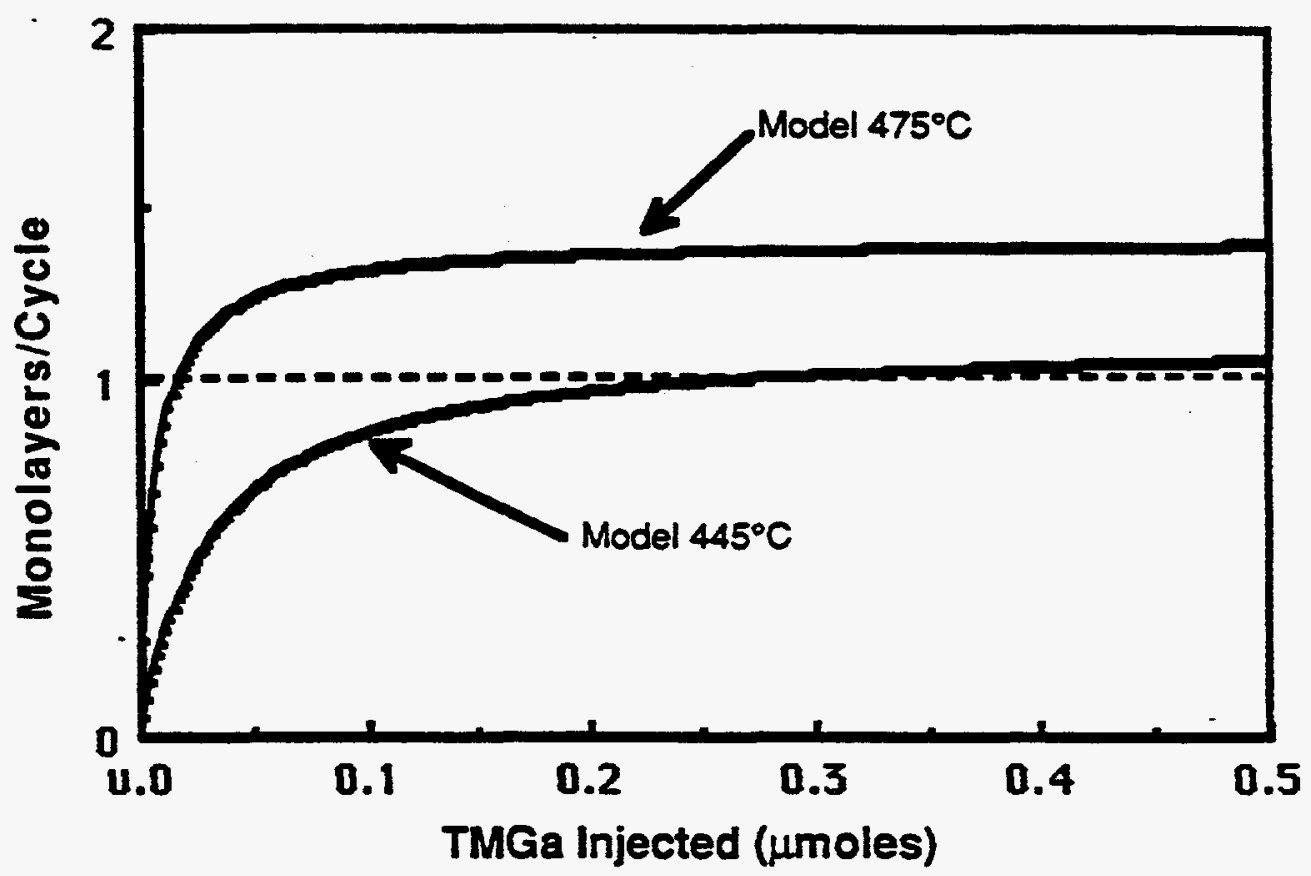

Figure 4.19

ALE growth rate as predicted by first order adsorption model with added deposition term accounting for decomposition of monomethylgallium to involatile gallium. 
Utilizing the rate constants shown in Table 4.2 which have been determined from previous results published in the literature13,14 we can then model the ALE deposition process. As shown in the calculated curves of Figure 4.19, we have been able to qualitatively explain the observed ALE growth behavior. We have also used the pyrolysis rate constant for the decomposition of arsine on the catalytic GaAs(100) surfaces with a first order adsorption model. As shown in Figure 4.20 below, there will be sufficient arsine adsorption for monolayer coverage until the temperature drops below $350^{\circ} \mathrm{C}$.

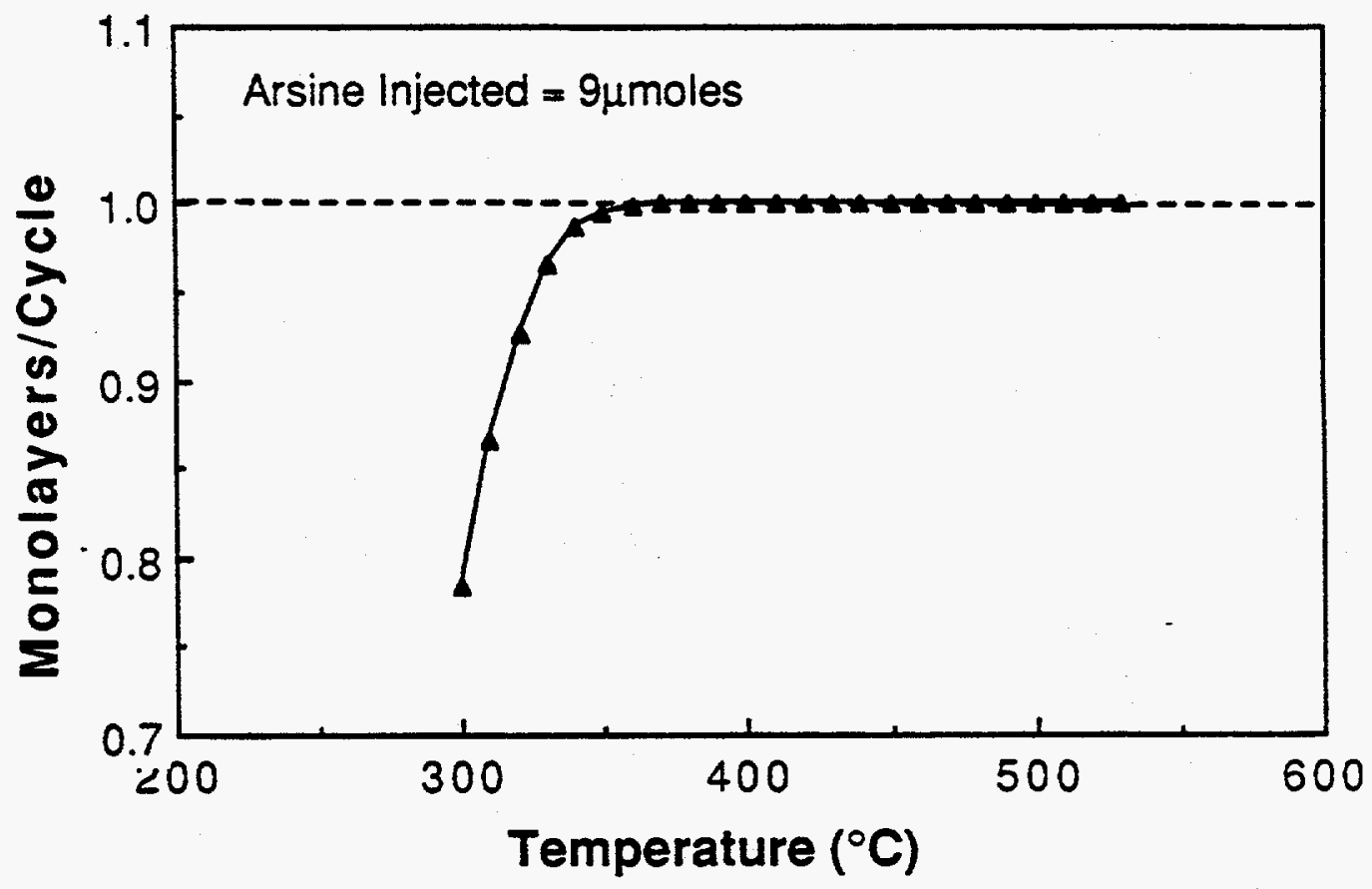

Figure 4.20

Monolayer coverage from arsine adsorption model. 


\begin{tabular}{|c|}
\hline Table 4.3 Rate Constants \\
\hline $\mathrm{k}_{\text {TMGa }}=1 \times 10^{18} \exp \left(-\frac{60 \mathrm{kcal}}{\mathrm{RT}}\right)$ \\
$\mathrm{k}_{\mathrm{MMGa}}=5 \times 10^{22} \exp \left(-\frac{77.5 \mathrm{kcal}}{\mathrm{RT}}\right)$ \\
$\mathrm{k}_{\text {des }}=333 \exp \left(-\frac{4.6 \mathrm{kcal}}{\mathrm{RT}}\right)$ \\
\hline Arsine on GaAs(100) Surfaces \\
\hline $\mathrm{k}_{\text {AsH }}=5.2 \times 10^{3} \exp \left(-\frac{18 \mathrm{kcal}}{\mathrm{RT}}\right)$ \\
\hline
\end{tabular}

\subsubsection{Boundary Laver}

The width of the boundary layer will determine the amount of gas heated to the reaction temperature. For laminar flow across a flat plate and assuming a typical velocity profile of the form:

$$
\mathbf{v}_{x}=a z+b z^{3}
$$

the thermal boundary layer can be expressed as: ${ }^{15}$

$$
\delta(x)=4.64 \sqrt{\frac{x\left(v^{\prime}\right)}{v_{\infty}}}
$$


where $\mathrm{x}$ is the distance along the plate from the leading edge, $\mathrm{V}_{\infty}$ is the average gas velocity, and $\mathbf{v}^{\prime}$ is the kinematic viscosity. For a hydrogen carrier gas stream at a temperature of $455^{\circ} \mathrm{C}$ the kinematic viscosity is $1.51 \mathrm{~cm}^{2} / \mathrm{sec}$. For our model we use the average boundary layer thickness across the substrate which we calculate to be $0.68 \mathrm{~cm}$ (See Appendix B for calculations).

\subsubsection{Kinetics of ALE in Nitrogen}

To test our kinetic model we have performed ALE growth of GaAs in an $\mathrm{N}_{2}$ ambient. The data of figure 4.21 show the dependence of the growth rate in $\mathrm{N}_{2}$ at $500^{\circ} \mathrm{C}$ as a function of the TMGa exposure. Also shown for comparison is the growth rate data for ALE growth in $\mathrm{H}_{2}$ at $455^{\circ} \mathrm{C}$. Note that the growth rate in $\mathrm{N}_{2}$ saturates at a thickness considerably less than the lower temperature growth in $\mathrm{H}_{2}$. This can be explained by the fact that the decomposition rate for TMGa in $\mathrm{N}_{2}$ is known to be suppressed relative to a $\mathrm{H}_{2}$ carrier gas because $\mathrm{N}_{2}$ is nonreactive with the TMGa and has a lower thermal conductivity. 16 As shown in figure 4.22 , there is approximately an $80^{\circ} \mathrm{C}$ temperature difference necessary in nitrogen to obtain the same pyrolysis rate constant. We expect that gas phase reaction rates are depressed in $\mathrm{N}_{2}$, but not surface. The data of figure 4.21 are consistent with a picture of the gallium methyl complex being chemisorbed in our process. 


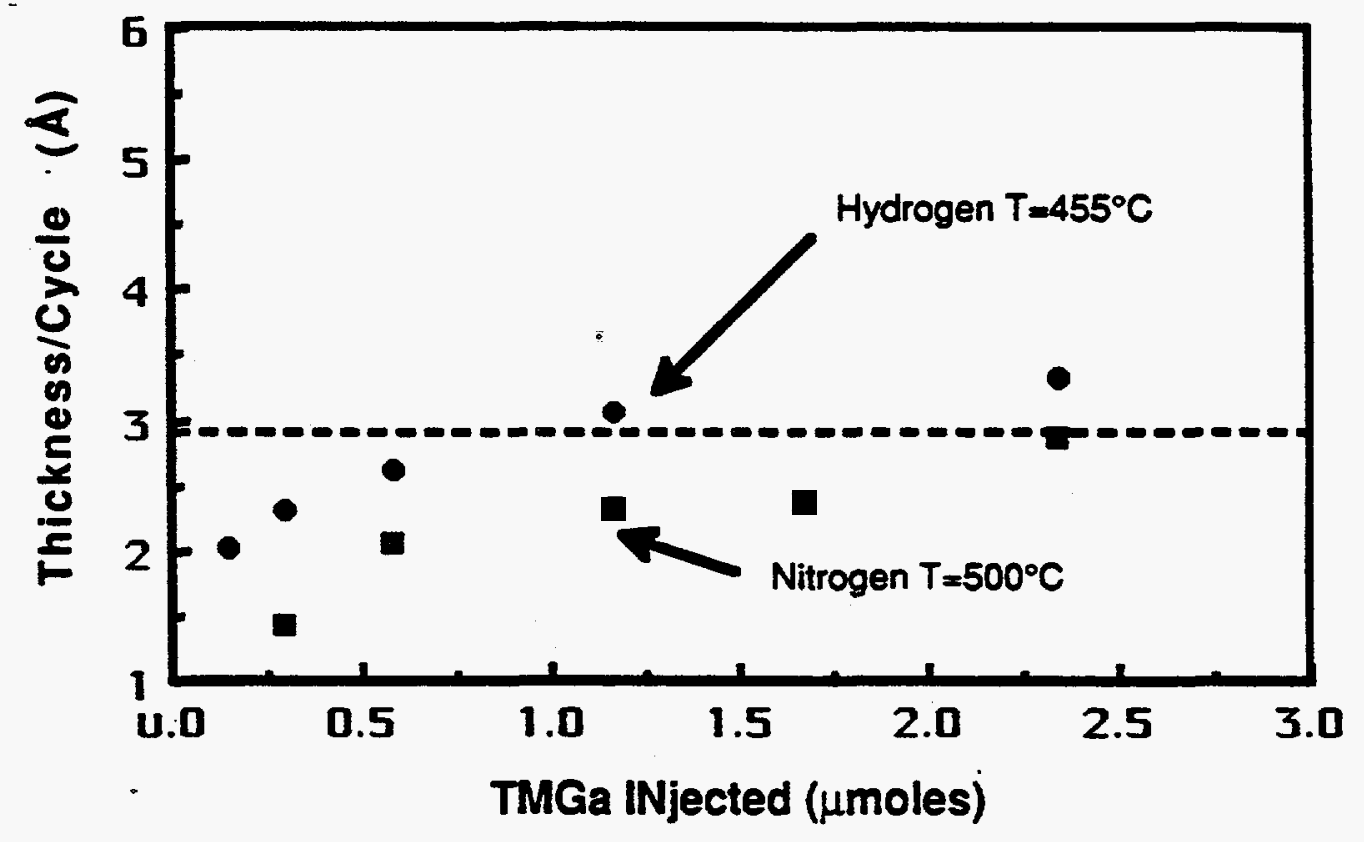

Figure 4.21

Growth rate of ALE GaAs layers in both hydrogen and nitrogen carrier gas ambient as a function of TMGa injected. 


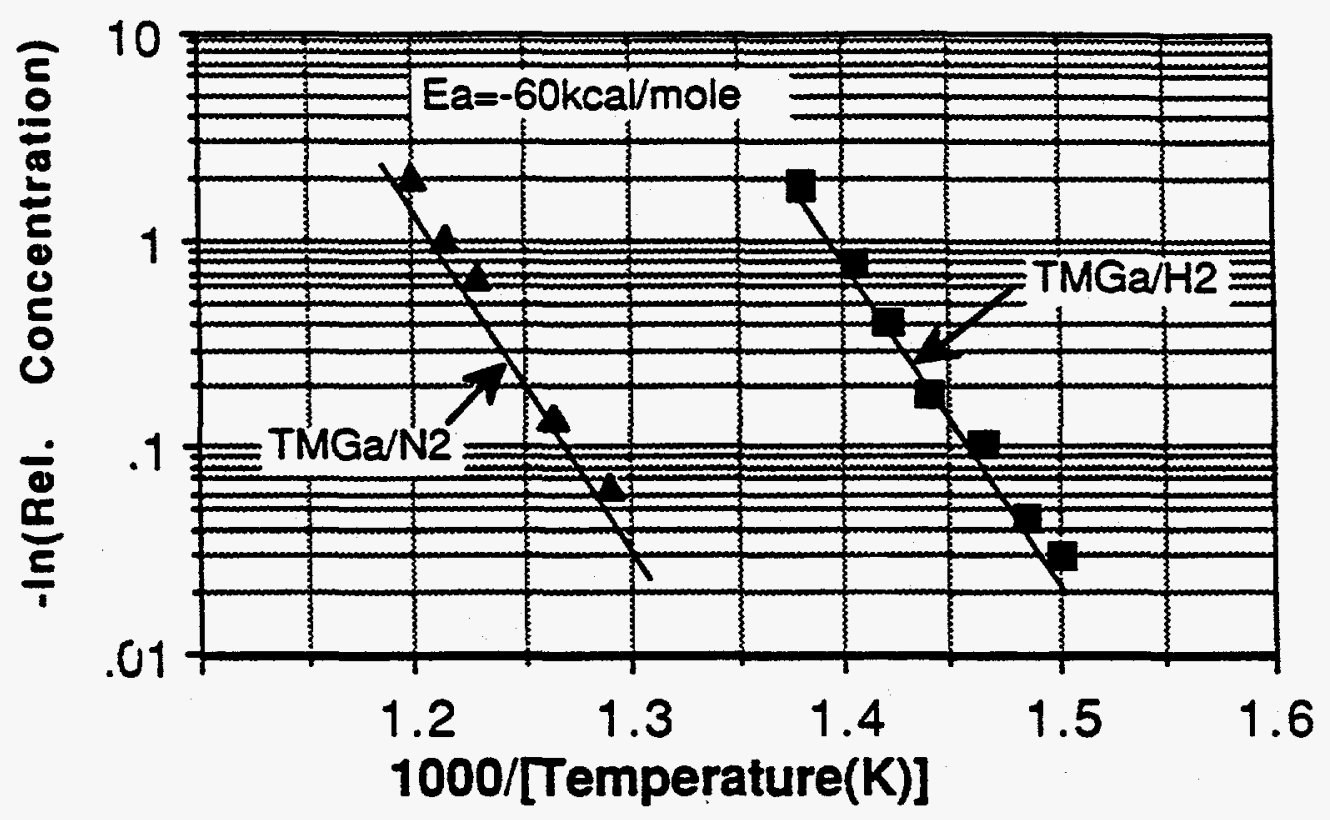

Figure 4.22

Logarithmic plot of the cracking rate of TMGa in nitrogen and hydrogen(data from Yoshida et al.). 17 Note the temperature difference necessary to obtain the same rate constant which corresponds to shift in ALE kinetic temperature range observed in Figure 4.21. 


\subsection{Conclusions}

Saturated monolayer growth of $\mathrm{GaAs}$ and AlAs has been observed by using metalorganic precursors at growth temperatures where kinetics of surface reactions dominate the deposition process. We have confirmed some of the inherent advantages of the ALE technique by demonstrating the "digital" nature of the deposition process and growing extremely uniform high quality quantum wells. The data presented distinctly show that ALE proceeds by: the chemisorption of a $\mathrm{Ga}\left(\mathrm{CH}_{3}\right) \mathrm{x}$ adsorbate (where $\mathrm{x}$ is most likely equal to 1 ), followed by the heterogeneous reaction of arsine with this adsorbate to complete the molecular monolayer. The kinetics for the ALE process have been determined and are shown to behave in accordance with a first order aclsorption model. The resultant process, carried out at atmospheric pressure, is compatible with conventional MOCVD and can be used to grow high quality materials and heterostructures by a hybridization of ALE and MOCVD. 


\section{REFERENCES-CHAPTER 4}

1 H. Kakibayashi and F. Nagata, Surface Sci, 74, 84 (1986).

2 L. J. Van der Pauw, Philips Res. Rept. 13, 13 (1958).

3 M. A. Tischler, N. G. Anderson, and S. M. Bedair, Appl. Phys. Lett. 49, 1199, (1986).

4 J. Nishizawa, T. Kurabayashi, H. Abe, and N. Sakuri, J. Electrochem. Soc. 134, 945 (1986).

5 T. F. Keuch and E. Veuhoff, J. Crystal Growth, 68,148 (1984).

6 E. Colas, R. Bhat, and B. J. Skromme,D-8, International Symposium on GaAs and Related Compounds, Atlanta, GA (1988).

7 S.Ito, T. Shinohara, and Y. Seki, J. Electrochem. Soc., 120, 1419 (1972).

8 J. Nishizawa, T. Kurabayashi, H. Abe, and A. Nozoe, Surface Sci., 185,249 (1987).

9 J. Nishizawa, T. Kurabayashi,H. Abe, and N. Sakurai, J. Electrochem. Soc. 134, 945 (1986).

10 J. Nishizawa, H. Abe and T. Kurabayashi, J. Electrochem. Soc. 132, 1197 (1985).

11 G.H. Geiger, D. R. Poirier, Transport Phenomena in Metallurgy, (Addison-Wesley Publishing Co., Menlo Park, CA 1980) p. 8.

12 M. G. Jacko and S. J. W. Price, Can. J. Chem. 41, 1560 (1963). 
13 S. P. DenBaars, B. Y. Maa, P. D. Dapkus, A. D. Danner and H. C. Lee, J. Crystal Growth, 77, 188 (1986).

14 Y. Aoyagi, A. Doi, S. Iwai, S. Namba, J. Vac. Sci. Technol. B5, 1460 (1987).

15 G.H. Geiger, D. R. Poirier, Transport Phenomena in Metallurgy, (Addison-Wesley Publishing Co., Menlo Park, CA 1980) p. 63.

16 M. Yoshida, H. Watanabe and F. Uesugi, J. Electrochem. Soc. 137, 677 (1985).

17 M. Yoshida, H. Watanabe and F. Uesugi, J. Electrochem. Soc. 137, 677 (1985). 


\section{CHAPTER 5 \\ ATOMIC LAYER EPITAXY OF \\ AlGaAs/GaAs HETEROSTRUCTURES AND QUANTUM \\ WELL LASERS}

\subsection{Introduction}

Recent research efforts have made it clear that heterostructure devices will play an important role in high speed electronics and communications systems. A heterostructure is a material in which a semiconductor with a narrow energy gap is sandwiched between two layers of a wider energy gap semiconductor. Heterostructures offer several physical benefits to electronic and opto-electronic devices such as optical waveguiding, selective absorption, and selective minority carrier injection and confinement. Heterojunction technology has made possible the realization of the continuous room-temperature operation of semiconductor injection lasers at low threshold currents. 1 This elevated the technology from a laboratory curiosity to what has become the key component of the fiber-optic communications systems. Also, the observation of the quantum size effect (QSE) in ultrathin heterostructures has given rise to a whole new generation of electronic and opto-electronic devices.

Advanced heterojunction device concepts that rely on ultra-thin layers and abrupt interfaces require unprecedented control and uniformity of layer properties for high yield implementation. Transistor structures such as high electron mobility transistors (HEMTs) and 
modulation doped field effect transistors (MODFETs) have also been shown to be dependent on monolayer level thickness variations. ${ }^{2}$ Threshold current density and lasing wavelength of quantum well injection lasers have been found to be extremely dependent upon the active layer thickness. 3 Therefore, active layer thickness uniformity in the range of one to two monolayers is necessary for these devices. Such control is difficult to achieve by conventional epitaxial techniques. Because atomic layer epitaxy operates with a self-limiting monoatomic growth mechanism, it appears to be especially well suited for the growth of these devices with the desired thickness control.

In this chapter we establish that ALE is capable of producing high quality GaAs/AlGaAs quantum well structures. Hybridization of the ALE and MOCVD growth technologies is demonstrated by utilizing ALE to grow the critical ultra-thin layers and MOCVD to grow the thicker AlGaAs regions. We show that under the proper growth conditions, extremely uniform layers of high-quality GaAs can be grown by ALE and that these layers can be incorporated into quantum wells that exhibit intrinsic photoluminescence (PL) at $8 \mathrm{~K}$ and $300 \mathrm{~K}$. We also present results on the first successful demonstration of a device fabricated by ALE in III-V semiconductors. Injection lasers with ALE quantum well active regions operate at room temperature with threshold current densities as low as $380 \mathrm{~A} / \mathrm{cm}^{2}$. 


\subsection{Quantum Well Properties}

\subsubsection{Calculation of Eigenvalue in finite potential well}

A quantum well is the term used to describe a double heterostructure in which the width of the smaller bandgap region is less than the DeBroglie wavelength of the charge carriers $\left(\lambda_{0}=280 \AA\right.$ in GaAs) ${ }^{4}$ in that material. Due to the quantum size effect in these structures, new discrete energy levels arise as shown in figure 5.1. These new discrete eigenvalues for the charged particles, electrons and holes, can be approximated by solving the Schrodinger equation with a finite square potential well:

$$
-\left(\frac{h^{2}}{2 m} \frac{\partial^{2}}{\partial z^{2}}+V\right) \psi=E \psi
$$

We derive the energy levels of an electron in a GaAs/AlGaAs quantum well with the barrier height given by the conduction band discontinuity " $\Delta E_{c}$." An analogous expression can be derived for the holes in the valence band of the well material with the use of the appropriate effective masses and barrier $\Delta E v$. For the electron, the Schrodinger equation becomes:

$$
\left(-\frac{h^{2} \partial^{2}}{2 m_{e}^{*} \partial z^{2}}-\Delta E_{c}\right) \Psi_{n}(z)=E_{e n} \Psi_{n}(z)
$$


where $\mathrm{m}^{*} \mathrm{e}$ is the effective mass of the electron, $\mathrm{h}$ is Planck's constant divided by $2 \pi$, and $E_{\text {en }}$ the energy level of the electron bound state or confined state.

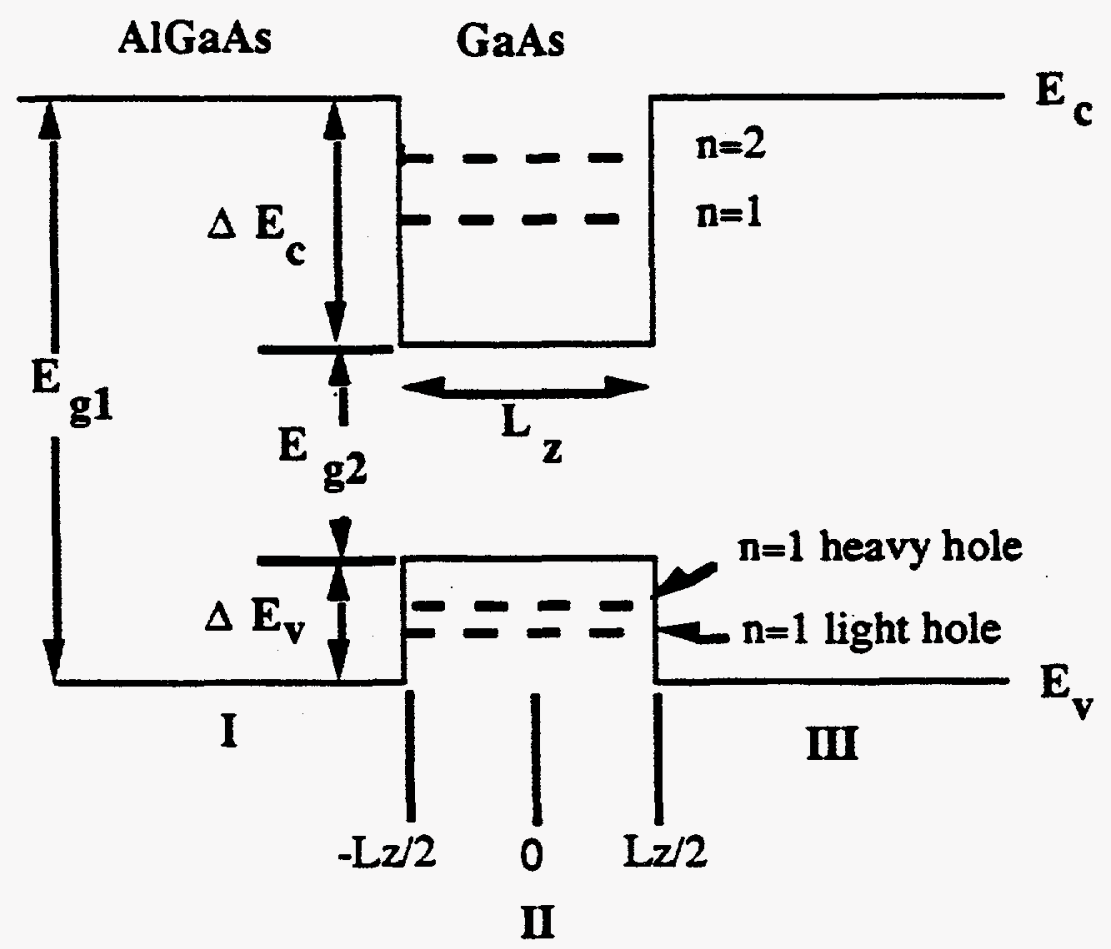

Figure 5.1

Energy band diagram for a GaAs/AlGaAs quantum well structure

The solutions of the eigenvalue equation in the three regions of the heterostructure are:

$$
\psi_{I}=\operatorname{Bexp}\left(k_{b}\left(z+\frac{L_{z}}{2}\right)\right) \quad . z \leq-\frac{L_{z}}{2}
$$




$$
\psi_{\text {III }}=B \exp \left(-k_{b}\left(z-\frac{L_{z}}{2}\right)\right) \quad z \geq \frac{L_{z}}{2}
$$

In the well region, the solutions are:

For even parity

$$
\Psi_{\mathrm{II}}=\mathrm{A} \cos \left(\mathrm{k}_{\mathrm{w}} \mathrm{z}\right) \quad|\mathrm{z}| \leq \frac{\mathrm{L}_{\mathrm{z}}}{2}
$$

$$
\text { For odd pairity } \quad \Psi_{I I}=A \sin \left(k_{w} z\right) \quad|z| \leq \frac{L_{z}}{2}
$$

$$
\begin{gathered}
\mathbf{k}_{w}=\frac{\sqrt{\left(2 m_{e}^{*}\left(\Delta E_{c}+E_{e n}\right)\right)}}{\bar{h}} \\
k_{b}=\frac{\sqrt{\left(2 m_{e}^{b}\left(E_{e n}\right)\right)}}{\bar{h}}
\end{gathered}
$$

Where $\mathrm{m}^{*}$ and $\mathrm{m}^{\mathrm{b}} \mathrm{e}$ are the effective mass of the electron in the well and barrier region,respectively.

There are two types of boundary conditions that can be employed for solution of the eigenvalues in quantum wells. R. Dingle 5 has obtained good agreement of experimental data with a model in which the wavefunction and its first derivative are matched at the interfaces. G. Bastard 6 has proposed that the most appropriate condition at the boundary is that the probability flux is continuous at the interfaces. The continuous flux model accounts for the effective-mass discontinuity at the 
interface. For our model we find that continuity of the solutions at the interfaces yield the best fit to both conventional MOCVD 7 and ALE experimental data. Matching solutions and first derivatives of equations 5.3 and 5.4 at the boundary $\left(z=L_{2} / 2\right)$ yields the transcendental equation for even parity :

$$
\tan ^{2}\left\lfloor\frac{\sqrt{\left(2 m_{e}^{*}\left(\Delta E_{c}+E_{e n}\right)\right)}}{\bar{h}}\left(\frac{L_{z}}{2}\right)\right\rfloor=\left(\frac{m_{e}^{b}}{m_{e}^{*}}\right)\left(-\frac{E_{e n}}{\Delta E_{c}+E_{e n}}\right)
$$

Similarly we can obtain the equation for odd parity:

$$
\cot ^{2}\left\lfloor\frac{\sqrt{\left(2 m_{e}^{*}\left(\Delta E_{c}+E_{e n}\right)\right)}}{\bar{h}}\left(\frac{L_{z}}{2}\right)\right\rfloor=\left(\frac{m_{e}^{b}}{m_{e}^{*}}\right)\left(-\frac{E_{e n}}{\Delta E_{c}+E_{e n}}\right)
$$

These equations are then solved numerically to obtain the eigenvalue $E_{\text {en }}$ of the electron bound states. For the eigenvalues of the hole bound states we solve similar eigenvalue equations which utilize the valence band discontinuity " $\Delta \mathrm{Ev}$ ". The PL emission energy is given by the transition from the $n=1$ electron level $E_{1 \text { en }}(L z)$ to the $n=1$ heavy-hole level $\left(E_{1 h h}(L z)\right.$ minus the exciton binding energy $E_{e x}$ :

$$
h v_{P L}=E_{g}+E_{1 e}\left(L_{z}\right)+E_{1 h h}\left(L_{z}\right)-E_{e x}\left(L_{z}\right)
$$

The materials parameters, energy dependence of the band gaps, and effective mass values for our model have been reported elsewhere. 8 We use an energy band discontinuity in our calculations in which $65 \%$ of the 
energy gap difference between the heterojunction materials is attributed to the conduction band discontinuity.

As shown in figure 5.2, there is good fit of the predicted emission energy in comparison to the expected well thicknesses in the range from

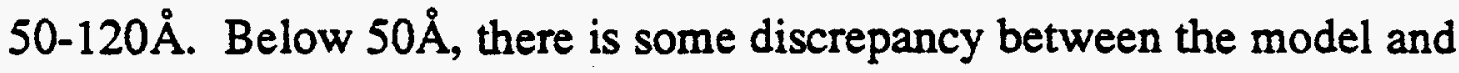
results. At this thickness tunneling of the wavefunction into the AlGaAs barriers becomes significant and further refinement of the wavefunction continuity model is necessary. Many quantum well thicknesses were measured by cleaved corner TEM measurements. Others were estimated by extrapolation of growth rates determined by SEM and angle lapping evaluation of thicker structures. Because there is good agreement of the model with measured well thicknesses at $70 \AA$, all the ALE quantum wells of varying parameters were grown with 25 cycles $(2.83 \AA$ × $25=70.75 \AA)$. Therefore, all of the $5 \mathrm{QW}$ thicknesses in the ALE study, shown in figure 4.3 of the previous chapter, can be measured relative to each other without the large discrepancies observed in smaller well structures. 


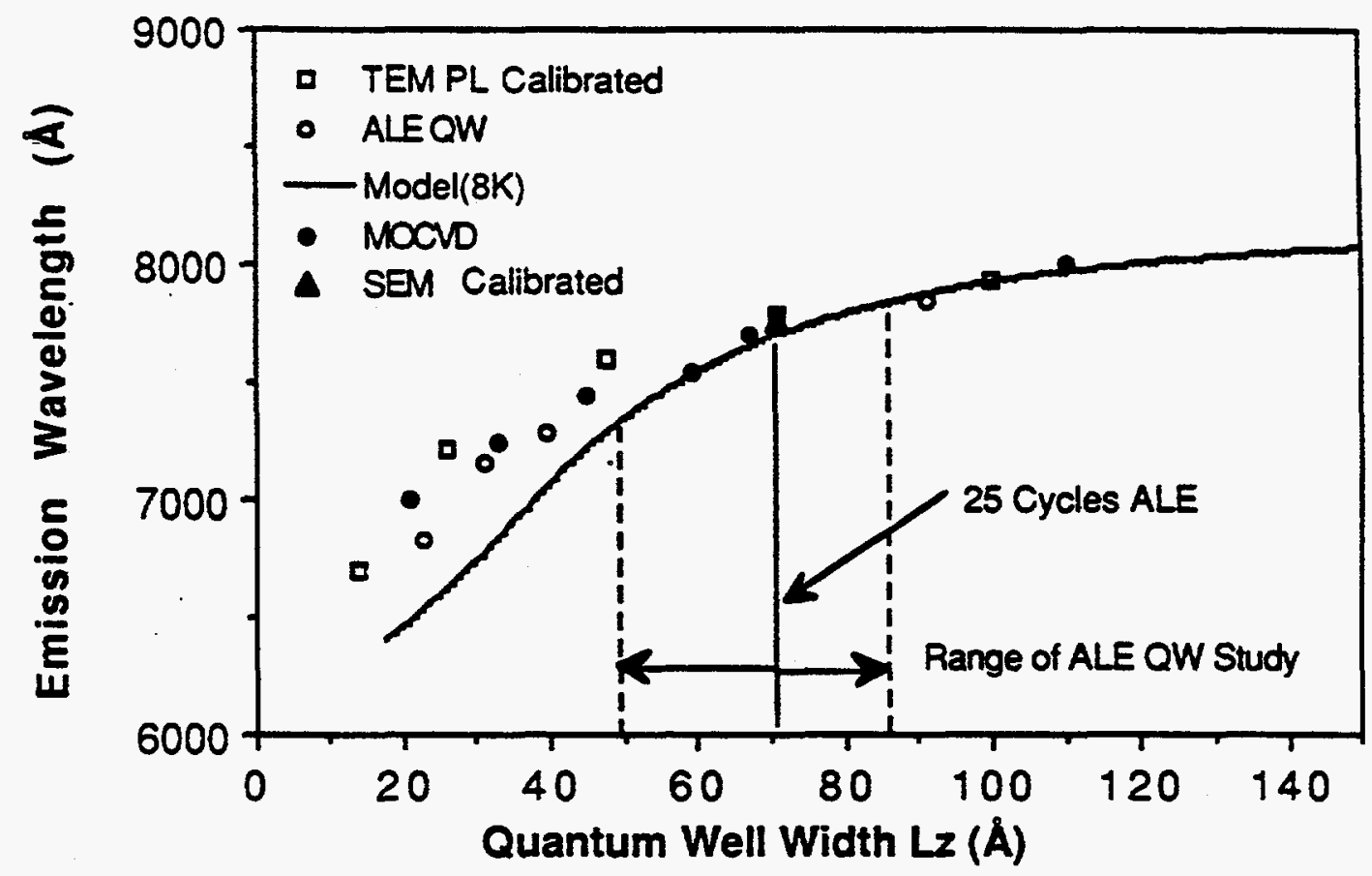

Figure 5.2

Shows the fit of experimental QW data to predicted emission wavelength 


\subsection{ALE Quantum Wells}

Single quantum wells are generally evaluated using low temperature photoluminescence (PL). The linewidth at these temperatures is a measure of the abruptness of the interface. Both the well width and the interface abruptness can be determined by modeling the SQW and comparing the predicted peak energy and linewidth with those measured experimentally. In order to evaluate the quality of ALE grown GaAs for quantum wells a five SQW test sample was studied, as shown schematically in figure 5.3. The five QW's of varying width are arranged such that the thinnest layer is grown last because it does not absorb luminescence from the wider QWs below which produce lower emission energy. The highest quality AlGaAs is produce by conventional MOCVD at high growth temperatures. Therefore, we grew the thick AlGaAs barriers at high temperatures $\left(750^{\circ} \mathrm{C}\right)$, the temperature was then lowered to the ALE temperature regime $\left(445-500^{\circ} \mathrm{C}\right)$, where the $\mathrm{QWs}$ were grown by the pulsed gas injection method. 


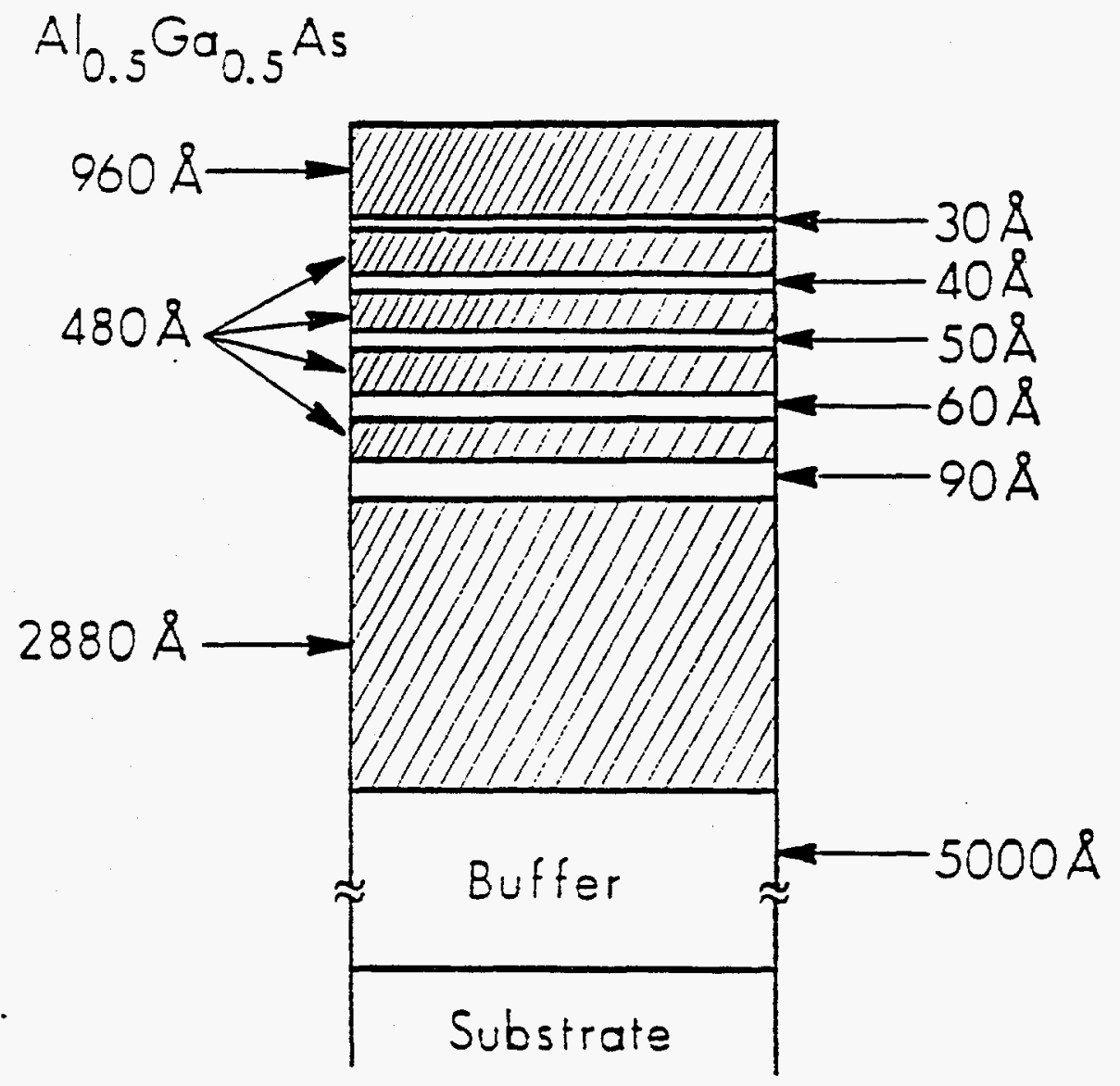

Figure 5.3

5 SQW sample grown for low temperature PL study. 


\subsubsection{PL Linewidths}

The quality of quantum wells formed by the ALE growth of the $\mathrm{GaAs}$ and MOCVD growth of $\mathrm{Al}_{0.5} \mathrm{Ga}_{0.5} \mathrm{As}$ at higher temperatures is demonstrated in the $8 \mathrm{~K}$ photoluminescence spectra as shown in figure 5.4. Note that all five quantum wells exhibit intrinsic radiative emission with narrow linewidths. The PL linewidths for each corresponding well thickness compare quite favorably with quantum wells grown in our laboratory or reported in the literature 9 by conventional MOCVD. The $1-3 \mathrm{meV}$ increase in the linewidth of the ALE grown quantum well in comparison to conventional grown MOCVD QWs is presumably caused by a small amount of impurity incorporation that may occur during the three-minute cool-down. The linewidth of the PL spectra is determined by a number of factors. At low measurement temperatures, the linewidth broadening is dominated by interface abruptness 10 and alloy clustering. The latter causes fluctuations in the composition that, in turn, can cause local potential differences. Other factors that may lead to linewidth broadening are electric fields from ionized impurities ${ }^{11}$ and band filling due to high carrier concentrations. 12 


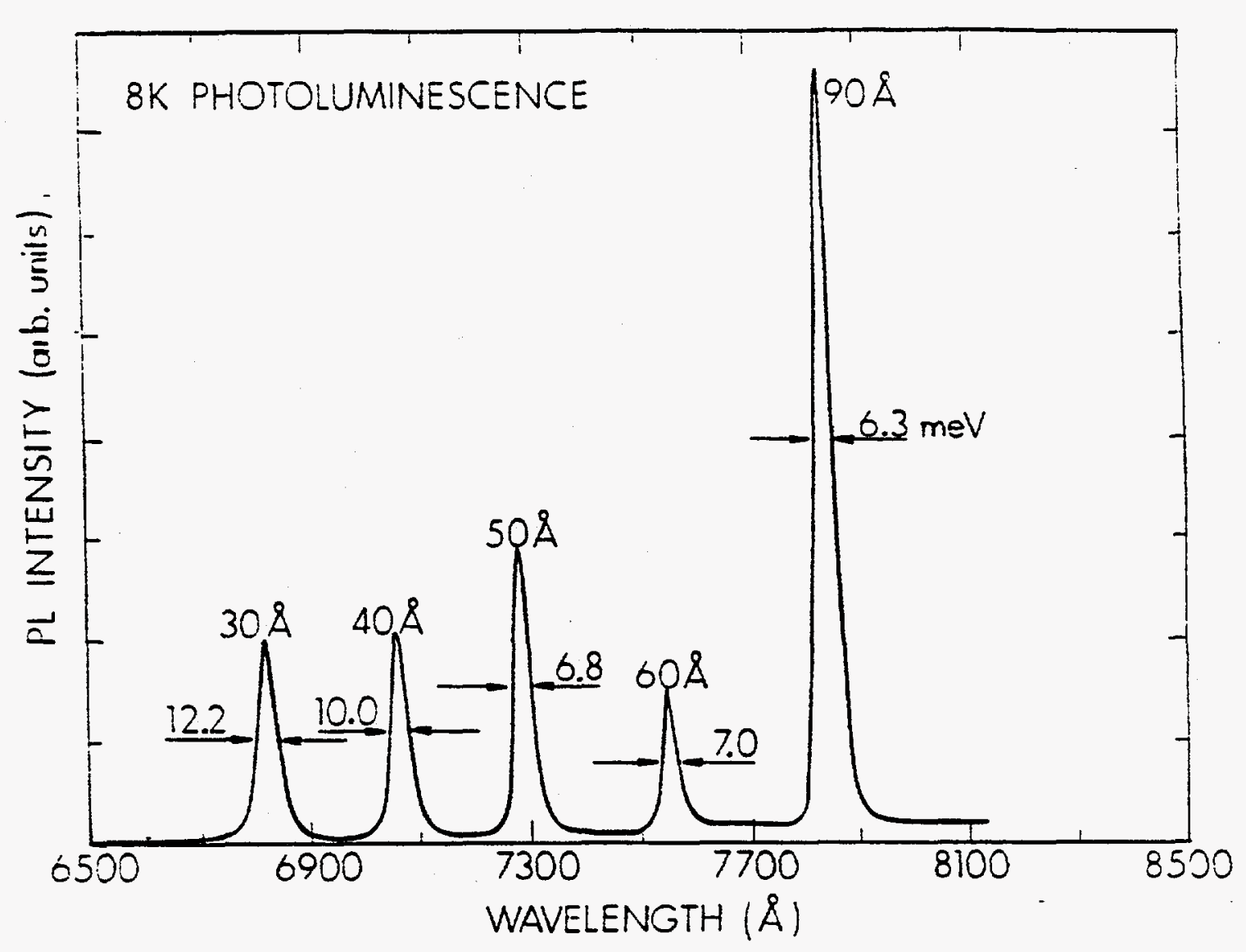

Figure 5.4

Low Temperature PL spectra of $5 S Q W$ exhibits narrow line intrinsic luminescence. 


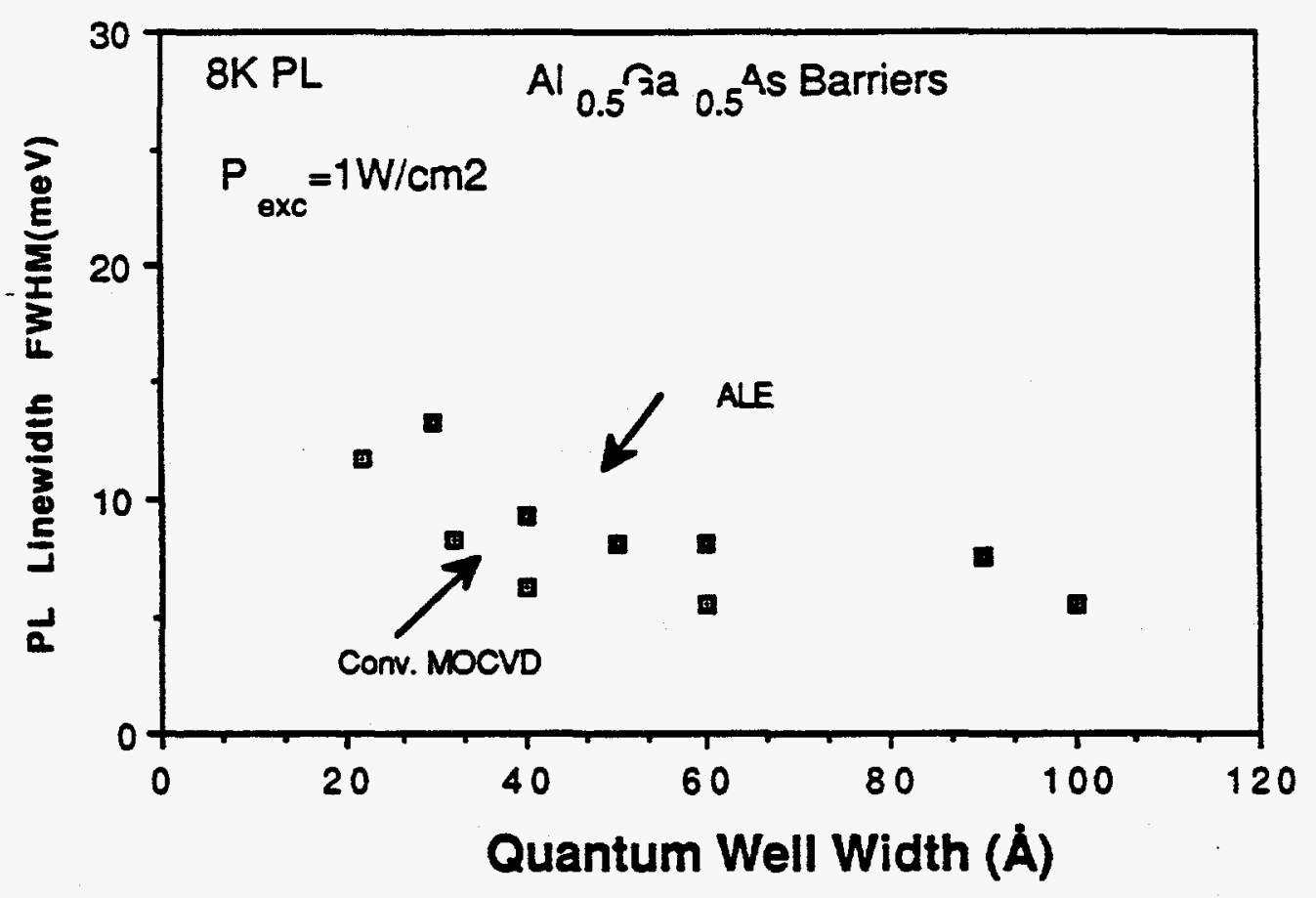

Figure 5.5

PL linewidth (FWHM) comparison of ALE and conventional grown MOCVD quantum wells 


\subsubsection{Interface Broadening}

In thin quantum well structures monolayer variations at the heterointerface will result in spectral broadening. This phenomena is illustrated in figure 5.5. Typically, the $\mathrm{QW}$ is not uniform in $\mathrm{L}_{z}$ within the layer, but is a assembly of lateral islands in the plane of the layer (See Figure 5.6). If the adjacent lateral island has a different thickness $L_{z}{ }^{*}$, it therefore yields a different PL emission energy. When the lateral extent, $\mathrm{W}$, of the island is larger the than Bohr diameter of the exciton, the PL spectrum exhibits multiple peaks. This has been observed in the PL spectrum of MBE-grown QW where long pauses at the heterointerface cause smoothness by surface migration and allows larger islands to grow.13 In the case where the lateral island size is smaller than the exciton diameter, an exciton experiences diff $\epsilon$ rent values of $L_{z}$ which causes spectral broadening. In this case, we can see the approximate effect of broadening $\Delta E$ caused by fluctuations in the well width by taking the derivative of the energy dependence of $\mathrm{E}$ for an infinite quantum well:

$$
\frac{\partial}{\partial L_{z}}(E)=\frac{\partial}{\partial L_{z}}\left(\frac{\bar{h}^{2}}{2\left(L_{z}\right)^{2} m_{e}^{*}}\right)
$$

Thus we can see the energy broadening will increase inversely with the cube of the QW width:

$$
\Delta \mathrm{E}_{\mathrm{PL}}=\left(\frac{\overline{\mathrm{h}}^{2}}{2 \mathrm{~m}_{\mathrm{e}}^{*}}\left[\frac{\Delta \mathrm{L}_{\mathrm{z}}}{\mathrm{L}_{\mathrm{z}}^{3}}\right]\right.
$$




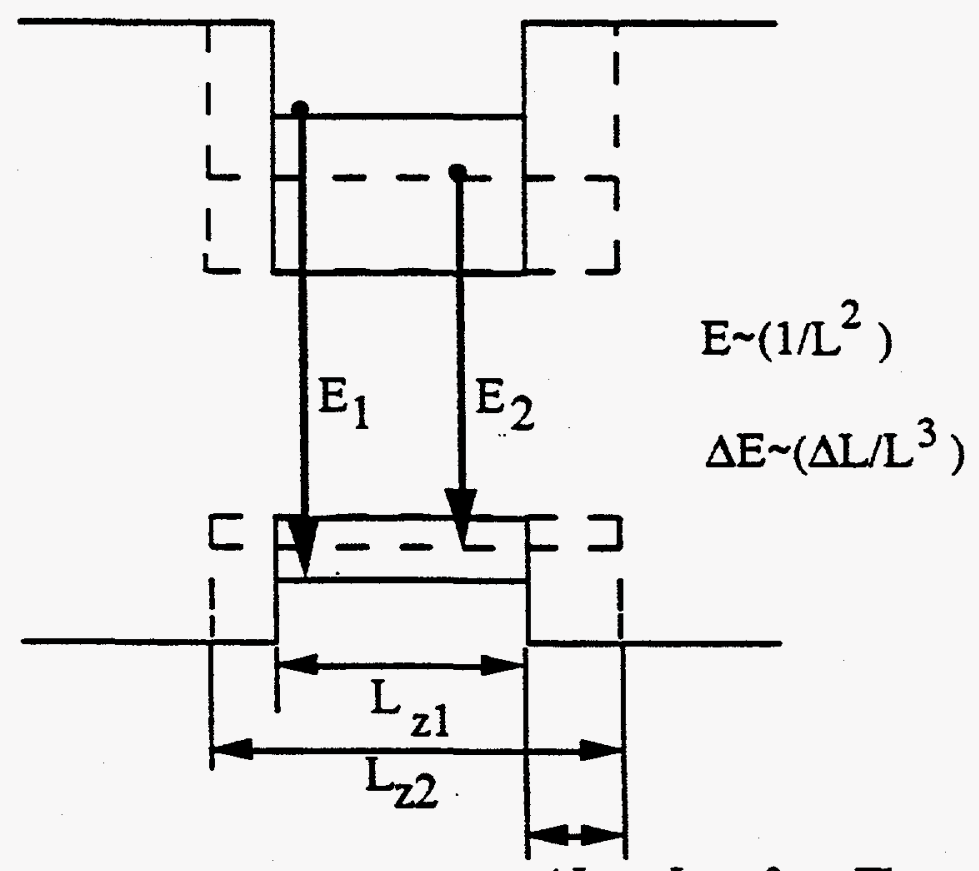

$\Delta \mathrm{L}_{\mathrm{z}}=$ Interface Fluctuations

Figure 5.6

Monolayer variations in the width of small quantum wells result in shifts in emission energy.

Singh et al. ${ }^{14}$ provide a more detailed model taking into account the finite potential barrier of $\mathrm{AlGaAs}$ and $\mathrm{GaAs}$ active region. Figure 5.8 shows the dependence of the spectral broadening as a function of the interface fluctuations and lateral island size. By utilizing this PL broadening model with a lateral island size of $100 \AA$, a well width fluctuation of one monolayer can account for the $12.2 \mathrm{meV}$ linewidth of the $30 \AA$ quantum well grown by ALE. 
Case 1( $\left.W_{Q W}>D_{\text {exciton }}\right)$

AlGaAs

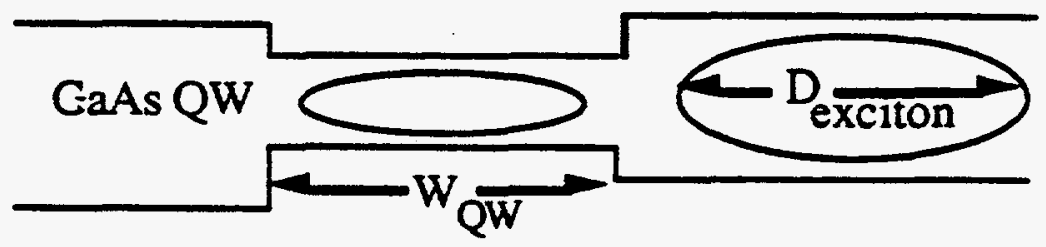

AlGaAs

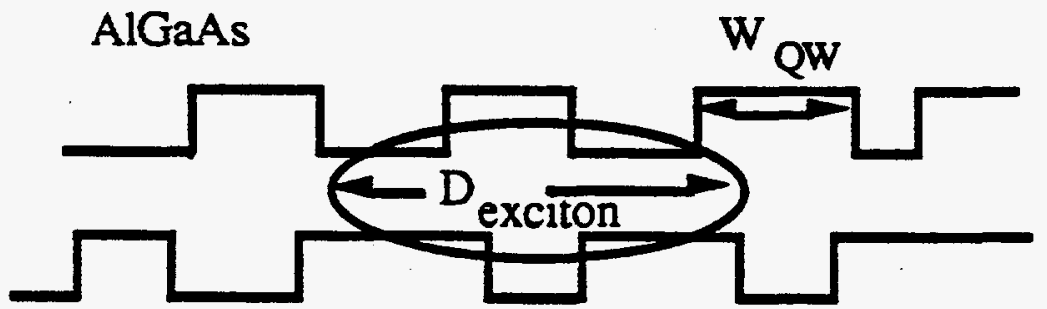

AlGaAs

Case $2\left(W_{\text {QW }}<D_{\text {exciton }}\right)$

Figure 5.7

Model of interface structure for interface island steps larger than the excitonic diameter (Case 1), and for interface islands steps smaller than excitonic diameter (Case 2). 
144

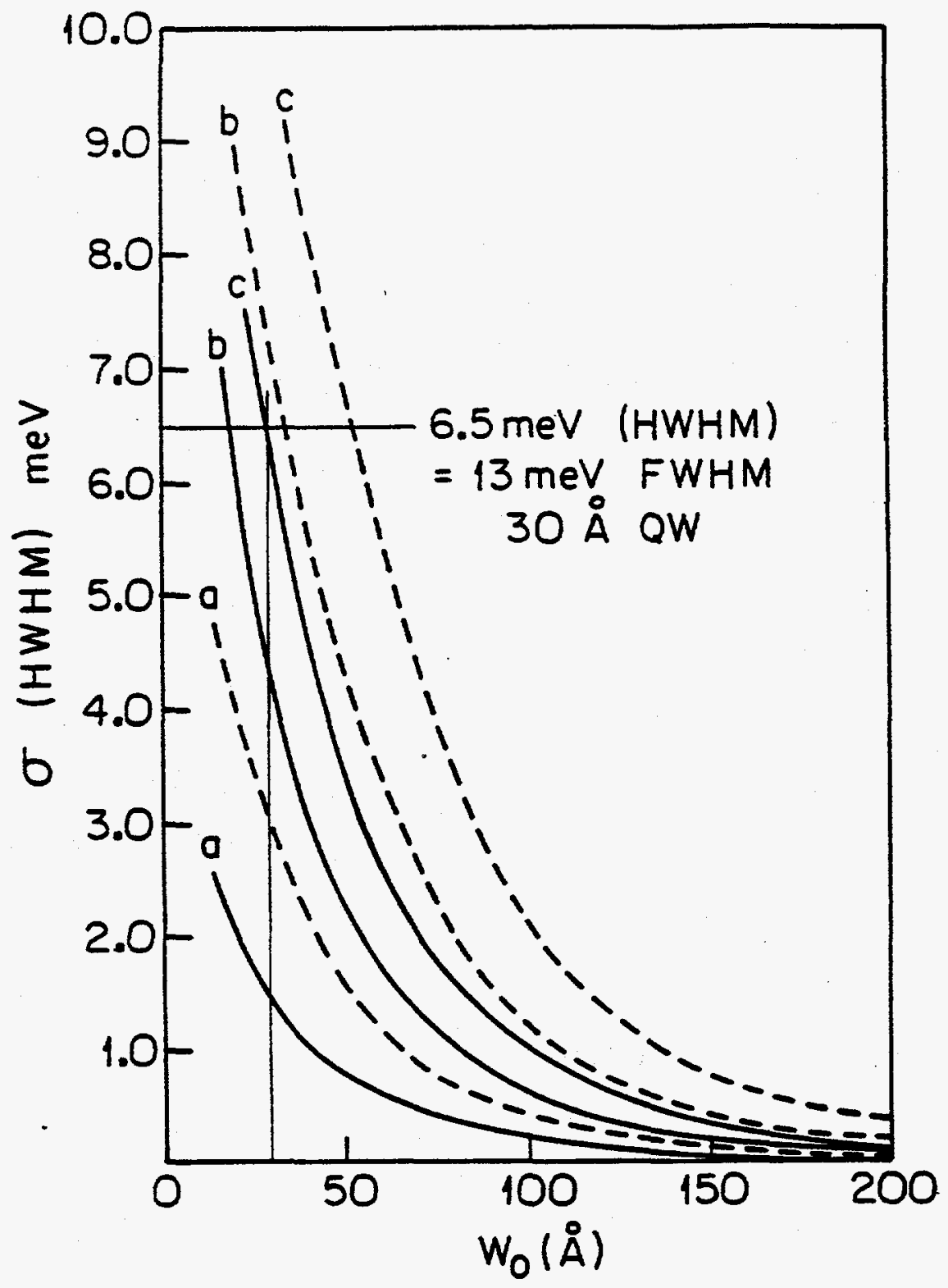

Figure 5.8

Half-width of PL emission as a function of well width $\mathrm{L}_{2}$ for one and two monolayer fluctuations. Lateral island size equals (a) $20 \AA$, (b) $80 \AA$, (c) $100 \AA$ (from Singh et al.). 14 Solid line indicates one monolayer interface fluctuations, whereas dashed line indicates two monolayer fluctuation. 


\subsubsection{Alloy Broadening}

Another factor contributing to PL line broadening is local fluctuations in the $\mathrm{Al}$ composition which will cause barrier height fluctuations. This effect is shown schematically in figure 5.9. S. B. Ogale et al. 15 have developed a rigorous quantum mechanical model which predicts that the PL linewidth will exhibit a $\left(\mathrm{L}_{2}\right)^{-1}$ dependence if compositional fluctuations are the dominant broadening mechanism. Figure 5.10 shows the comparison between theory and experimental data for several growth techniques. From this figure we can determine that if compositional fluctuations are the dominant broadening mechanism the observed PL linewidths in the ALE grown QWs can be accounted for by only a $1.0 \%$ compositionally fluctuation in the AlGaAs barrier layers. Therefore, the PL spectra of ALE grown SQWs shown in figure 5.4 suggest that the interface abruptness and quality of the quantum wells has not been significantly altered by interrupting the growth to lower the temperature to ALE conditions. 


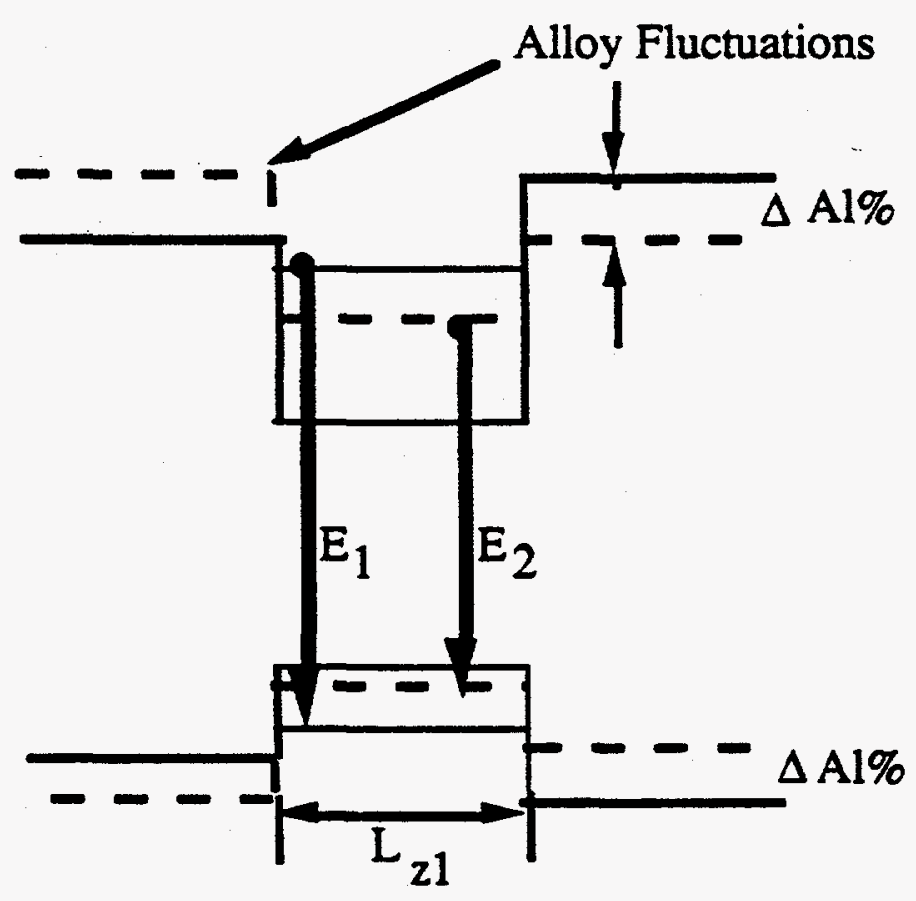

Figure 5.9

Local fluctuations in Aluminum concentration cause broadening of the PL emission. 


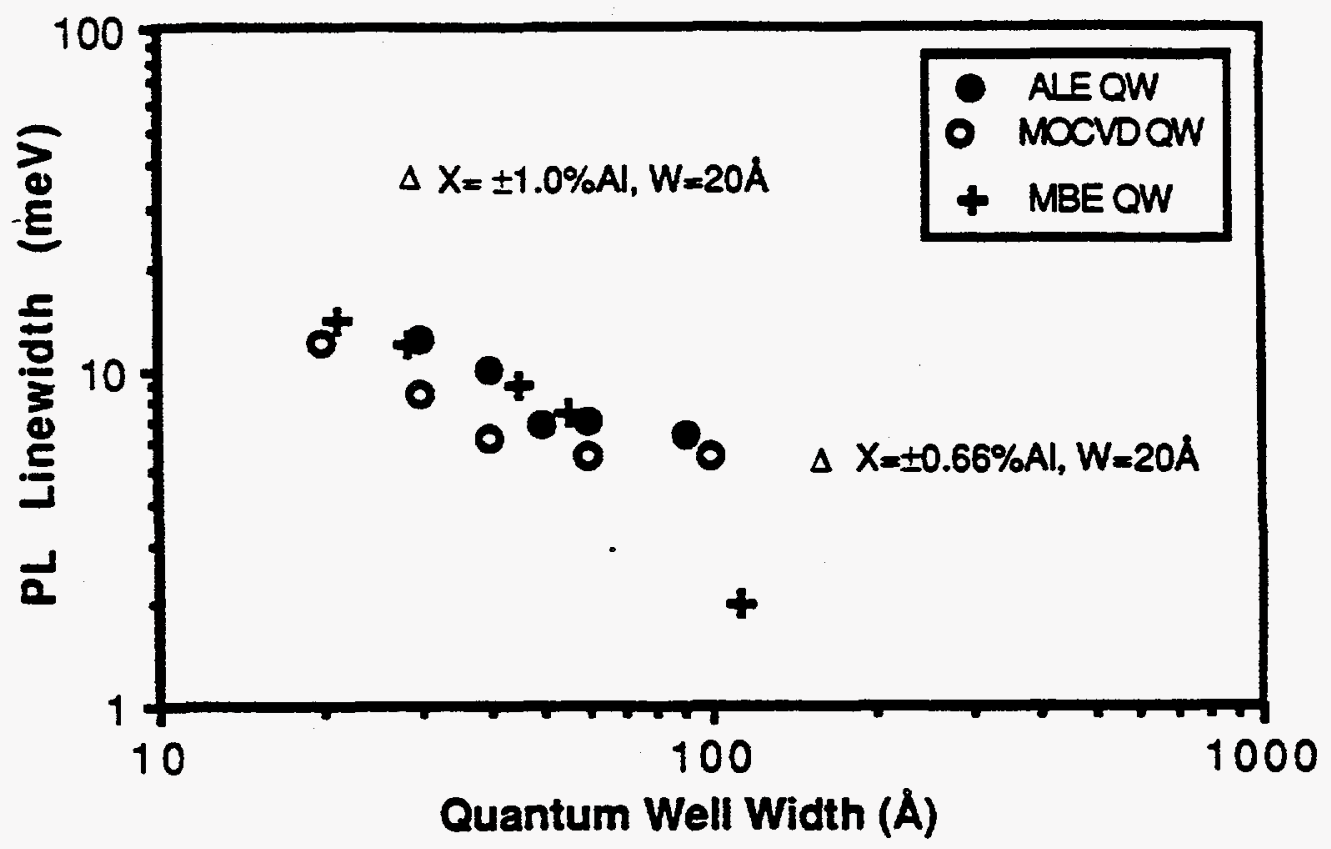

Figure 5.10

The measured dependence of PL linewidth as a function of QW width for various growth methods. Solid lines indicate predicted PL broadening caused by fluctuations in Al composition with $33 \% \mathrm{Al}$ barriers and $20 \AA$ lateral islands widths from S. B. Ogale et al. 16 


\subsubsection{Optimization of ALE Quantum Well Luminescence}

Several factors influence the quality of luminescence from quantum wells. Poor AlGaAs quality and heavy impurity incorporation in the wells will lower luminescence efficiency. Oxygen incorporation will act as a non-radiative recombination site in both AlGaAs and GaAs. For the ALE growth of quantum wells the most important parameter we have identified that effects the photoluminescence quality is the lack of surface coverage caused by an inefficient arsine exposure. Figure 5.10 shows the detrimental effects produced by reducing the arsine exposure below the amount required to obtain perfect surface coverage " $\Theta=1$." Not only does the luminescence efficiency decrease but also there is significant increase in the linewidth of the emission. The increased linewidth is due to the homogeneous line broadening caused by increased impurity incorporation in these layers. The most likely impurity being incorporated is carbon caused by incompletion of the proposed surface reaction between arsine and the gallium adsorbate.

$$
\mathrm{GaCH}_{3}(\mathrm{ads})+\mathrm{AsH}_{3}-\mathrm{GaAs}_{(\mathrm{s})}+\mathrm{CH}_{4}(\mathrm{v})+\mathrm{H}_{2}(\mathrm{v})
$$

Therefore, sufficient arsine amounts and time of exposures are necessary for high quality GaAs by ALE. 


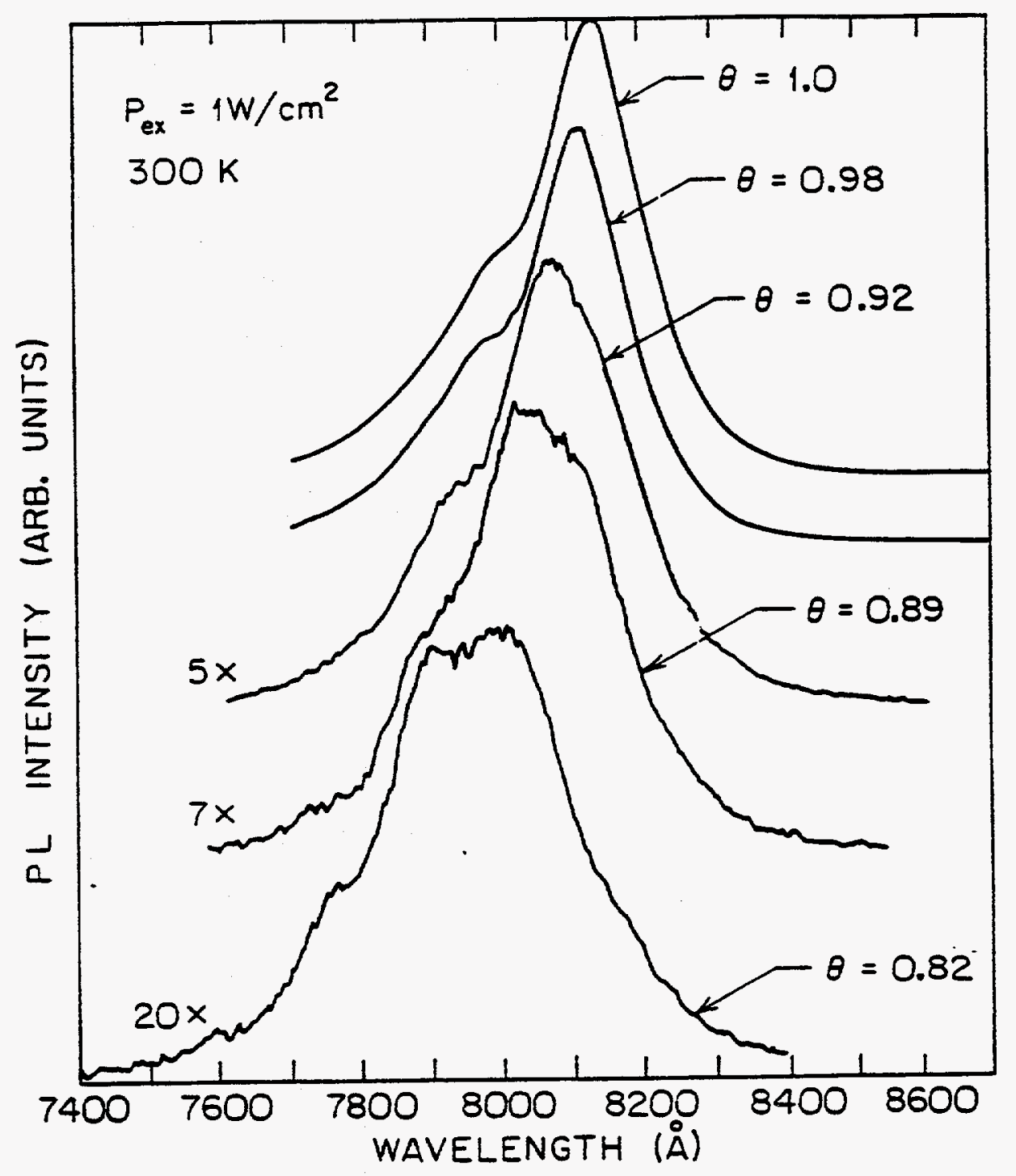

Figure 5.11

Room-temperature PL spectra of 5 SQWs grown with varying surfaces coverages of arsine at $455^{\circ} \mathrm{C}$. Note that the insufficient arsine coverages reduce the PL efficiency and increase the PL FWHM linewidths. 
The effect of growing quantum wells with various surface coverages of TMGa on the PL spectra is shown in figure 5.11. Interestingly enough the linewidths of the PL spectra at lower TMGa surface coverages appear to be narrower than for the thicker QWs. Therefore for coverages greater than one there appears to an increase in the impurity incorporation. It is likely that putting to much TMGa on the surface blocks the surface exchange reaction between the arsine and the methyl groups. The observation that the thinnest well has the narrowest linewidth might indicate that the exchange reaction is more efficient when the surface is not completely covered by the gallium adsorbate. This is of great interest in comparison to the recent results of $E$. Colas et al. 17 who obtained the best electrical results of ALE GaAs, $\mu_{77}=35,000 \mathrm{~V} / \mathrm{cm}^{2} \mathrm{sec}$ and $3 \times 10^{15} \mathrm{~cm}^{3} \mathrm{n}$-type, by growing at $1 / 2$ monolayer per cycle. 


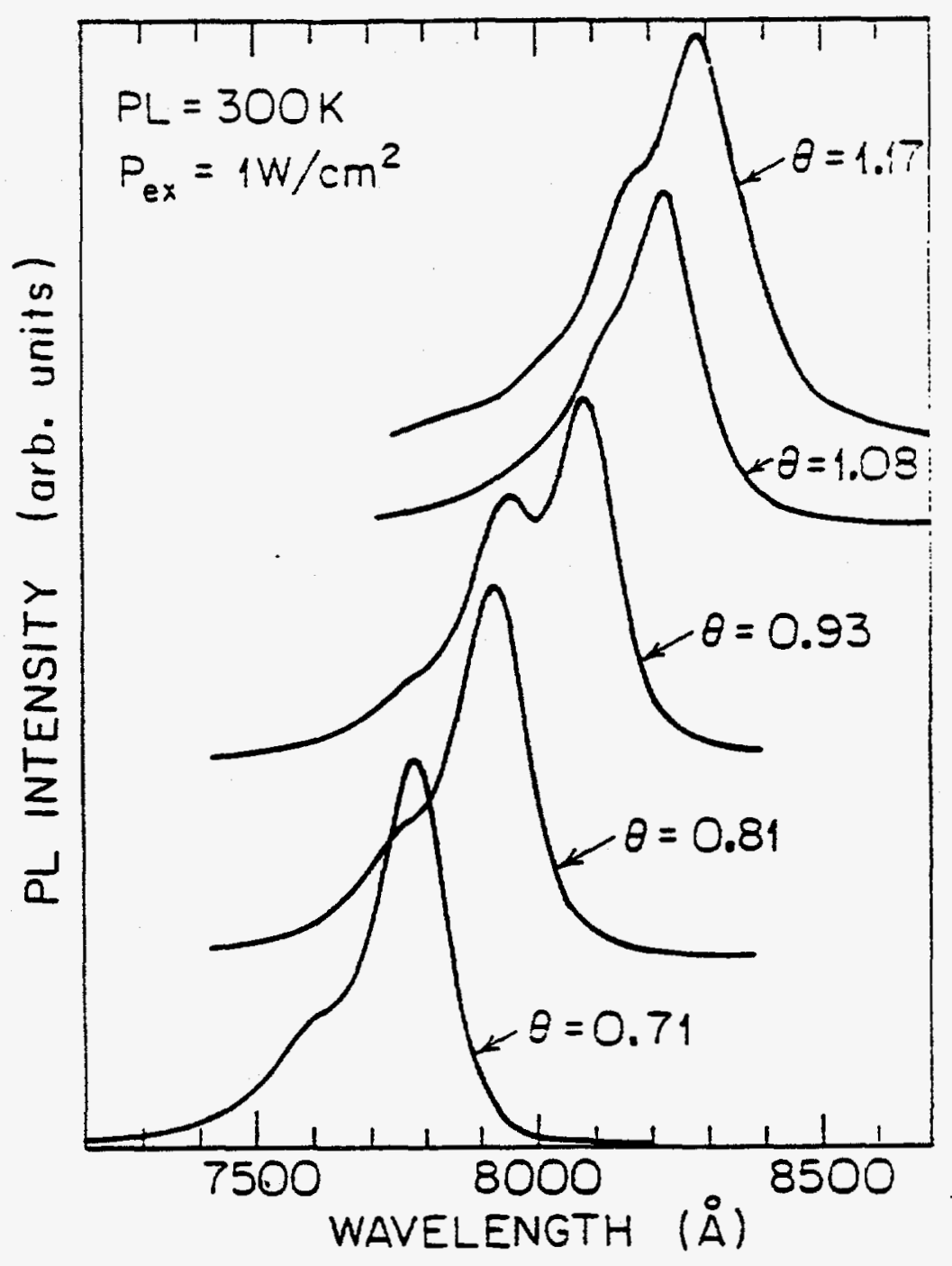

Figure 5.12

Room-temperature PL spectra of 5 SQWs grown with varying surfaces coverages of $\mathrm{TMGa}$ at $455^{\circ} \mathrm{C}$. 


\subsubsection{AlGaAs/GaAs heterostructures grown entirely by ALE}

ALE of AlGaAs has been achieved by injecting the TMAl both concurrently, and separately from the TMGa pulse. As was shown in the ALE of AlAs in figure 4.6 the deposited thicknesses show the expected saturated growth results. However, as shown in figure 5.13, extrinsic luminescence is observed from the structures in which ALE was also used to grow the barriers. This indicates that further optimization of the process is needed. Luminescence is only observed from structures in which the TMAl is injected simultaneously with the TMGa pulse. We have also grown a $70 \AA \mathrm{Al} 0.2 \mathrm{Ga0} .8 \mathrm{As} \mathrm{QW}$ by $\mathrm{ALE}$. The barriers for this well were $\mathrm{Al} 0.5 \mathrm{Ga0} .5 \mathrm{As}$ grown by conventional MOCVD at $750^{\circ} \mathrm{C}$. The expected emission energy for this well is approximately $6200 \AA$. As we can see PL spectrum in figure 5.14, a small peak exist at the expected energy but lower energy extrinsic luminescence dominates the spectrum.

There are several reasons accounting for the low quality of ALE grown AlGaAs compared to ALE grown GaAs. First, aluminum is thermodynamically a more reactive element than gallium. The reaction of oxygen with an aluminum arsenide surface is 100 times that of a $\mathrm{GaAs}$ surface. 18 We have also observed this in the growth on conventional MOCVD QW structures in which we studied the effect of long pauses at the GaAs and AlGaAs interfaces. ${ }^{19}$ As shown in figure 5.15 low temperature PL from these studies indicate that pauses at the AlGaAs 
interface at high temperatures lead to significant linewidth broadening. This linewidth broadening is attributed to the increased impurity incorporation on the AlGaAs interface.

Another factor that might cause the poor luminescence is the fact that the Al-C bond is much stronger in TMAl than TMGa and thus more carbon might be incorporating in ALE AlGaAs. Using TEAl compounds appears to be a solution to this problem. The aluminum to ethyl bond is weaker than the aluminum to methyl bond 20 and thus less carbon incorporation will occur. By using TEAl in the flow modulated epitaxy scheme Makimoto et al.21 have grown high quality MQW structures at temperatures as low as $500^{\circ} \mathrm{C}$. Low-temperature PL spectra of their QWs with $\mathrm{Al} 0.2 \mathrm{Ga0} .8 \mathrm{As}$ barriers are as narrow as 10.9 and $4.2 \mathrm{meV}$ for $18 \AA$ and $68 \AA$ wells, respectively. 


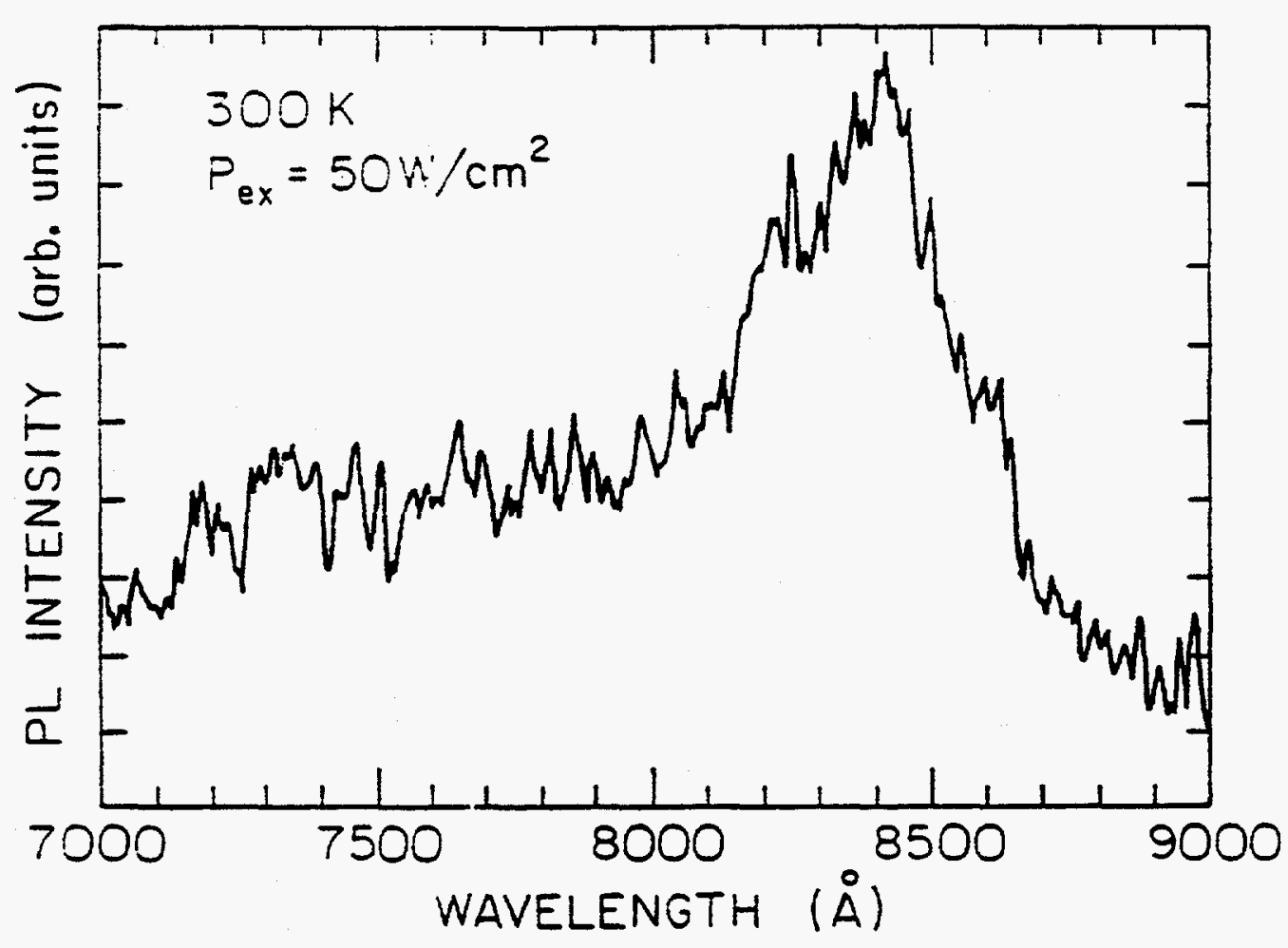

Figure 5.13

PL spectrum of a GaAs/AlGaAs QW structure in which ALE was also used to grow the AlGaAs barrier layers. 


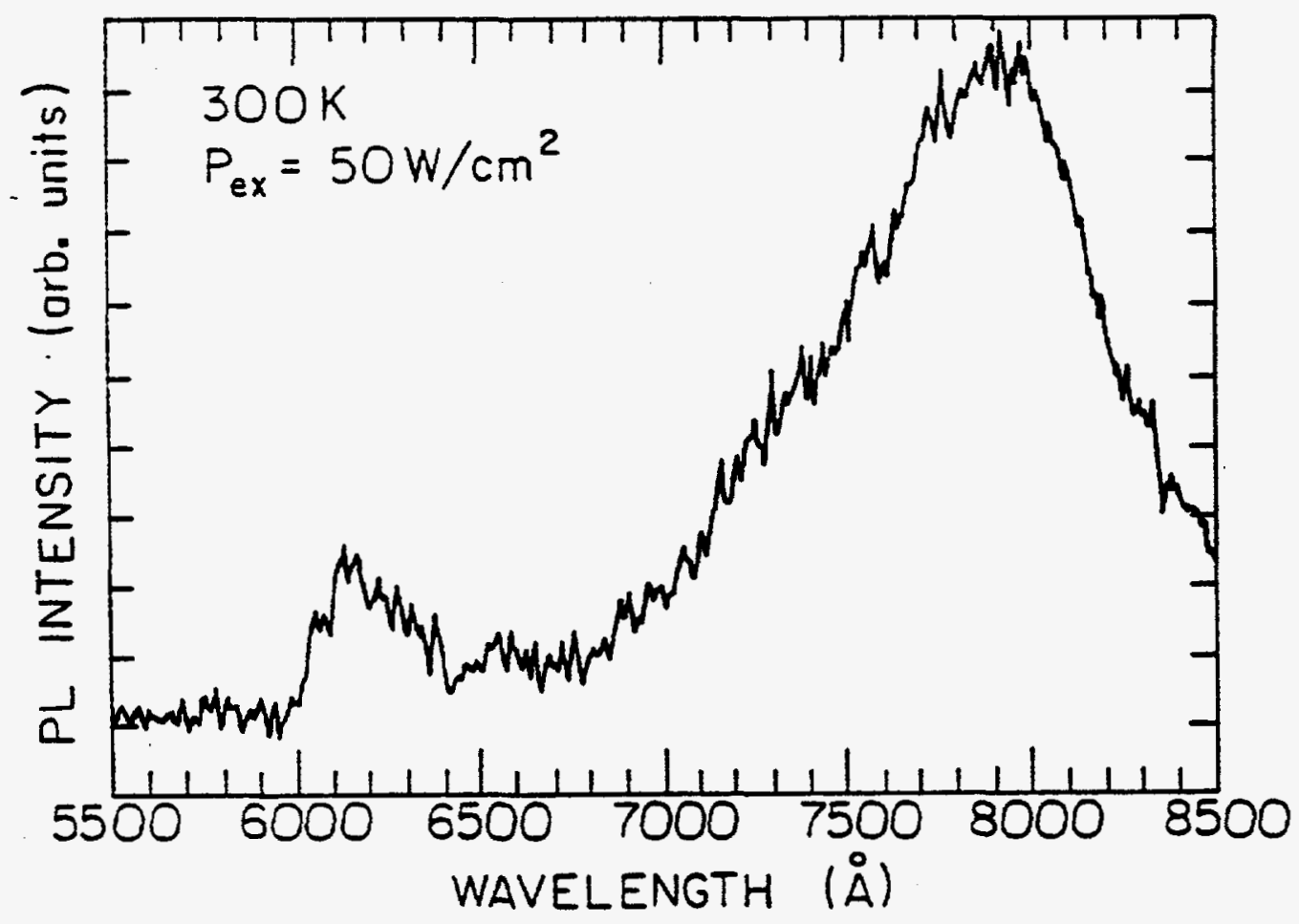

Figure 5.14

PL spectrum of a $70 \AA$ QW with $A l_{0.2} \mathrm{Ga0} .8$ As ALE active region and $\mathrm{Al} 0.5 \mathrm{Ga0} .5 \mathrm{As}$ barriers. 


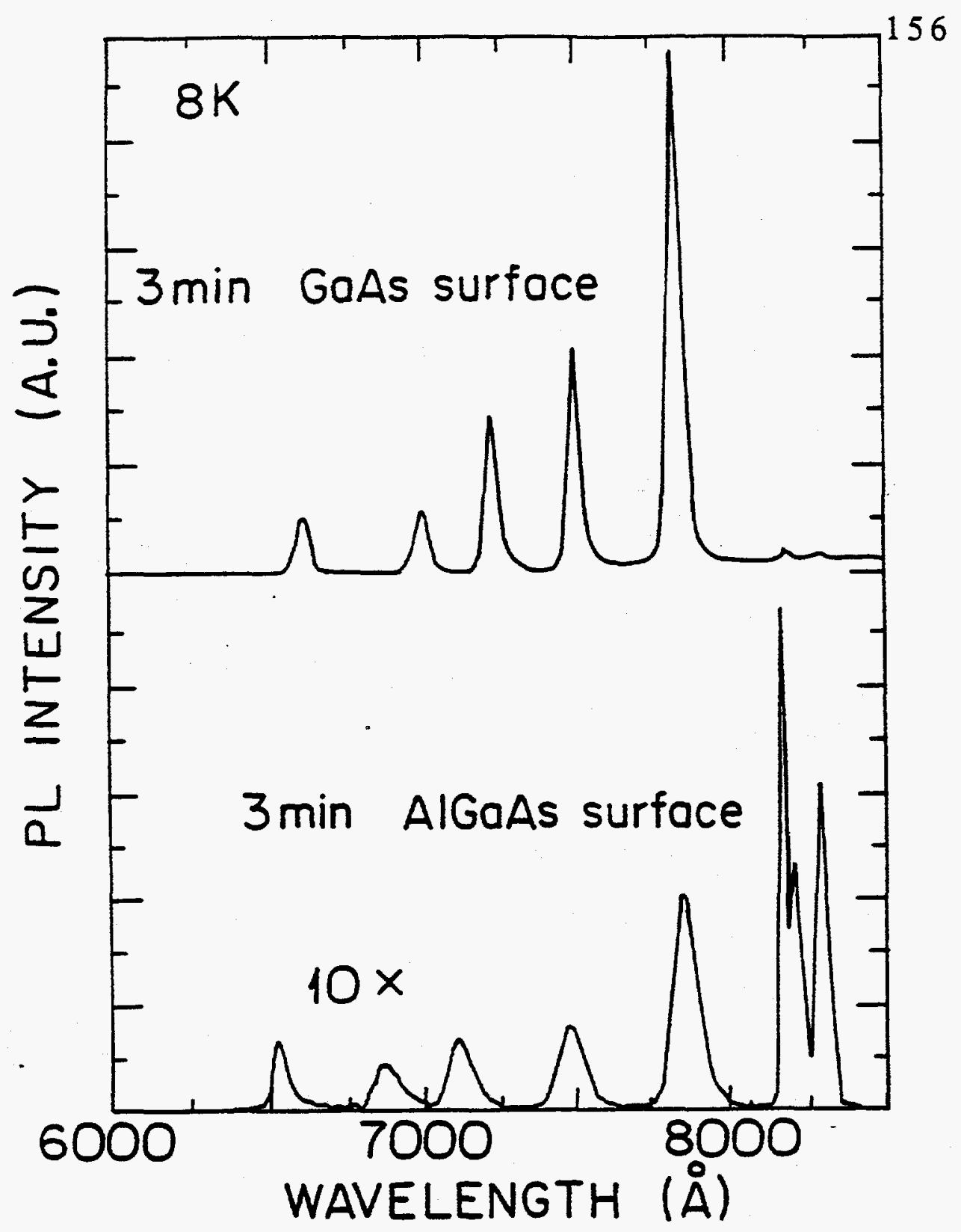

Figure 5.15

Low temperature PL spectra for five MQW structure in which 3 minute pauses at the AlGaAs interface lead to increased impurity incorporation. The uppermost spectrum shows narrow PL from QWs in which the pause was at the GaAs interface only. The lower spectrum shows the extrinsic broadened PL from QWs in which the pause was at the AlGaAs interface only. 


\subsection{Quantum Well Injection Lasers}

Quantum wells, as well as devices incorporating these structures require the ability to produce thin layers with abrupt interfaces. An example of one device is the quantum well injection laser. In this device the threshold current density and lasing wavelength are strongly dependent upon the active layer thickness in the 10-60 $\AA$ range. 22 We have demonstrated that monolayer uniformity is achievable by the ALE growth technique as shown in figure 4.8. Therefore, as a test vehicle for utilizing ALE in device structures, we have applied ALE to the growth of $\mathrm{GaAs} / \mathrm{AlGaAs}$ quantum well injection lasers in which the critical thickness GaAs active region is grown by ALE.

Quantum well heterostructures offer several benefits to operation of semiconductor lasers. Extremely low currents threshold current operation are possible because of the step-like 2-dimensional density of states of the quantum well structure. Discrete energy levels exist in the 2dimensional density of states, thus more efficient utilization of injected carriers at a given energy level is achieved as shown in figure 5.16. This also reduces the temperature dependence of the threshold current density, 23 which typically plague conventional double heterostructure lasers. Carrier confinement in the QW enhances the gain coefficient and reduces the threshold current. 24 For parabolic bands with infinite barriers it can be shown that within each subband there exist a constant density of states, per unit area. 


$$
\rho(E) d E=\left(\frac{m_{n}^{*}}{\pi h^{2}}\right) d E
$$

For a GaAs/AlGaAs heterostructure the density of states in the conduction band can be shown to be: 25

$$
\rho_{c}(E)=\left(\frac{m_{c}^{*}}{\operatorname{sh}^{2} L_{z}}\right)\left(\operatorname{INT}\left(\frac{E-E_{c}}{\Delta E}\right)^{1 / 2}\right)
$$

Thus, the density of states in a heterostructure is a quasi-twodimensional density of states which appears as step like function that is asymptotic to the 3-D density of states as $\mathrm{Lz}$ becomes large (See figure 5.16). 


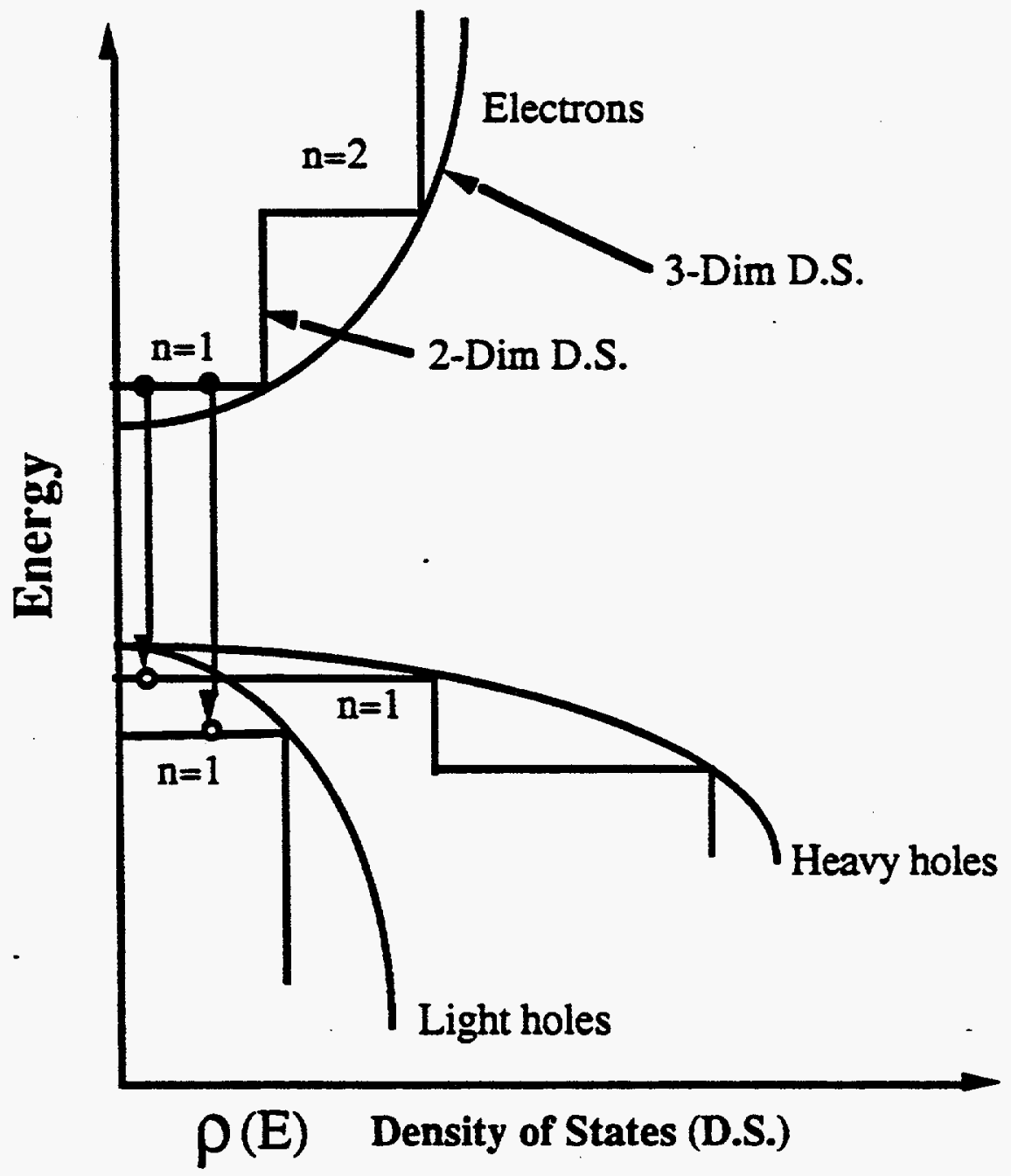

Figure 5.16

Quantum well laser offer discrete transitions energy levels because of the step-like 2-Dimensional density of states. 


\subsubsection{Growth and Fabrication of ALE Quantum Well Injection Laser}

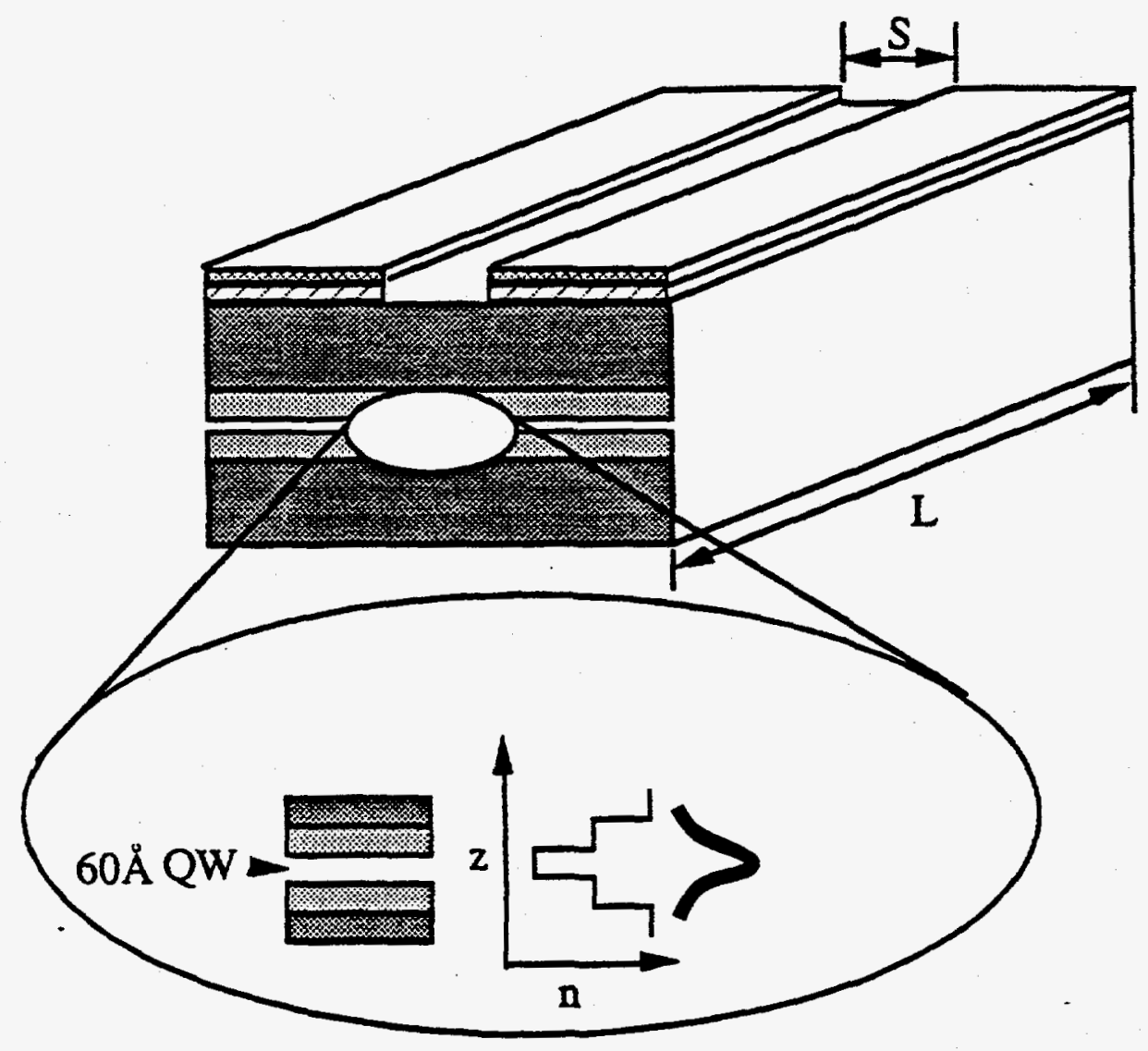

Figure 5.17

Schematic diagram of separate confinement (SCH) heterostructure laser employing $60 \AA$ quantum well active region grown by ALE.

Injection laser device structures were formed in three stages in a single growth run. The first stage of growth consists of growing a $0.2 \mu \mathrm{m}$ $\mathrm{n}+\mathrm{GaAs}$ buffer layer, a $2 \mu \mathrm{m}$ n-type Al0.5Ga0.5As cladding layer and an $600 \AA$ undoped $A l 0.25 \mathrm{Ga0} .75 \mathrm{As}$ confinement layer. These layers were 
grown at a temperature of $750^{\circ} \mathrm{C}$. The temperature was lowered to either $455^{\circ} \mathrm{C}$ or $485^{\circ} \mathrm{C}$ and the $60 \AA$ quantum well active region was grown by ALE. The final stage consisted of the growth of an undoped confinement layer, a p-type cladding layer and a p+ cap layer. These three layers had the same compositions as the layers grown in the first stage and were also grown by conventional MOCVD at $750^{\circ} \mathrm{C}$. Laser devices were processed by depositing $100 \mu \mathrm{m}$ wide $\mathrm{Cr}$-Au stripes and AuGe-Ni contacts on the pand n-sides of the device, respectively. Injection laser device chips were fabricated by cleaving $400-800 \mu \mathrm{m}$ long bars with the cleaved faces perpendicular to the stripe direction. Figure 5.22 shows a micrograph of a typical laser chip. The other sides of the device were scribed and broken to prevent laser operation on internal bounce modes. A schematic illustration of a separate confinement heterostructure $(\mathrm{SCH})$ quantum well laser is shown in figure 5.17 .

\subsubsection{Device Results}

The ALE QW injection laser device chips were tested in a pressure mount fixture under pulsed conditions. The current was applied in $0.3 \mu \mathrm{sec}$ long pulses at a repetition rate of $10 \mathrm{kHz}$. A typical spectrum from a broad area device under spontaneous emission and laser operation is shown in figure 5.18. An expanded view of the laser spectra shows a several longitudinal modes appear above threshold (See figure 5.19). The mode spacing is determined by wavelengths satisfying the phase condition:

$$
m\left(\frac{\lambda_{0}}{\bar{n}}\right)=2 L
$$


where $m=1,2,3 . .$, and $L$ is the cavity length, and $\bar{n}$ the refractive index. It can then be shown the mode spacing will be given by: 26

$$
\Delta \lambda_{0}=\left(\frac{\lambda_{0}^{2}}{2 \bar{n} \amalg\left[1-\left(\frac{\lambda_{0}}{\bar{n}}\right)\left(\frac{d \bar{n}}{d \lambda_{0}}\right)\right]}\right)
$$

A summary of the threshold current densities obtained for $Q W$ injection lasers grown by ALE in comparison to the best conventional MOCVD grown lasers in our lab is shown in Table 5.1. Graded index separate confinement heterostructures (GRIN-SCH) yield the lowest threshold current densities for lasers grown by both techniques. Figure 5.20 illustrates the difference in grading between the SCH and GRIN-SCH laser structures. Increased optical confinement $(\Gamma)$ along with the pseudoelectric field created by the parabolic graded region have been used to explain the lower threshold currents necessary in the GRIN-SCH structures. 27 This can be shown by examination of the threshold current density $J_{\text {th }}$ equation for a double heterostructure $(D H)$ laser which is given as: 28

$$
\mathbf{j}_{\text {th }}=\frac{\mathrm{J}_{\mathrm{o}} \mathrm{d}}{\eta_{\mathrm{i}}}+\frac{\mathrm{d}}{\eta_{\mathrm{i}} \beta \Gamma} \alpha_{\mathrm{i}}+\frac{\mathrm{d}}{\eta_{\mathrm{i}} \beta \Gamma}\left(\frac{1}{\mathrm{~L}} \ln \left(\frac{1}{\mathrm{R}}\right)\right)
$$

where $\eta$ is the internal quantum efficiency, $\Gamma$ the optical confinement factor, $L$ the cavity length, $\beta$ is the gain constant, and $d$ the active region thickness. The internal loss term is given by:29 


$$
\alpha_{i}=\Gamma \alpha_{f c}+(1-\Gamma) \alpha_{f c B}+\alpha_{s}+\alpha_{c}
$$

where $\alpha_{\mathrm{fc}}$ is the background free carrier concentration in the active region $\alpha_{\mathrm{fcB}}$ is the concentration in the barriers as scattering loss, and $\alpha_{\mathrm{c}}$ the coupling losses.

Threshold current densities as low as $380 \mathrm{~A} / \mathrm{cm}^{2}$ were observed for the ALE GRIN-SCH structures. 30 Differential quantum efficiencies as high as $26 \%$ per facet were observed in the output power versus drive current curves (See figure 5.21). Equivalent structures with optimized grading and doping of the confinement regions grown in our laboratory by conventional vertical MOCVD have threshold currents as low as 160 $\mathrm{A} / \mathrm{cm}^{2}$. These low thresholds were only achieved after careful calibration of the AlGaAs doping level and the width of the parabolic grading. From the internal loss equations we can see that reduction of the free carrier concentrations in the active regions and the $\mathrm{AlGaAs}$ barriers will reduce the losses. We thus anticipate threshold current densities in the range 200$400 \mathrm{~A} / \mathrm{cm}^{2}$ for lasers with ALE-grown active regions with further optimization of the cladding region doping.

Table 5.1 Threshold Current Densities

\begin{tabular}{|lll|}
\hline Structure & Vertical MOCVD & ALE \\
\hline SCH & $400 \mathrm{~A} / \mathrm{cm} 2$ & $640 \mathrm{~A} / \mathrm{cm} 2$ \\
\hline GRIN-SCH & $160 \mathrm{~A} / \mathrm{cm} 2$ & $370 \mathrm{~A} / \mathrm{cm} 2$ \\
\hline
\end{tabular}




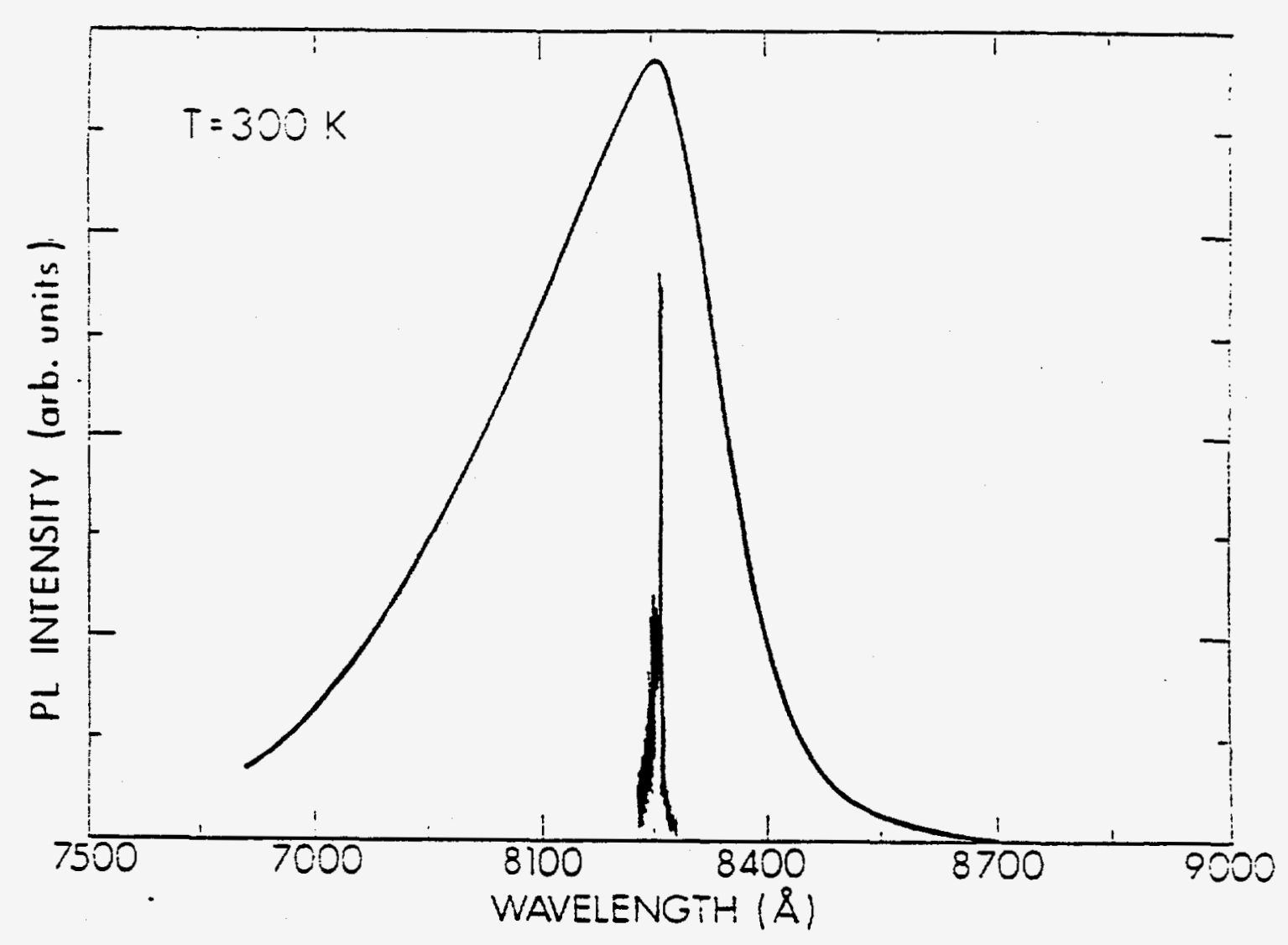

Figure 5.18

Spontaneous and laser emission spectrum from a separate confinement quantum well laser with GaAs QW active region by ALE. 


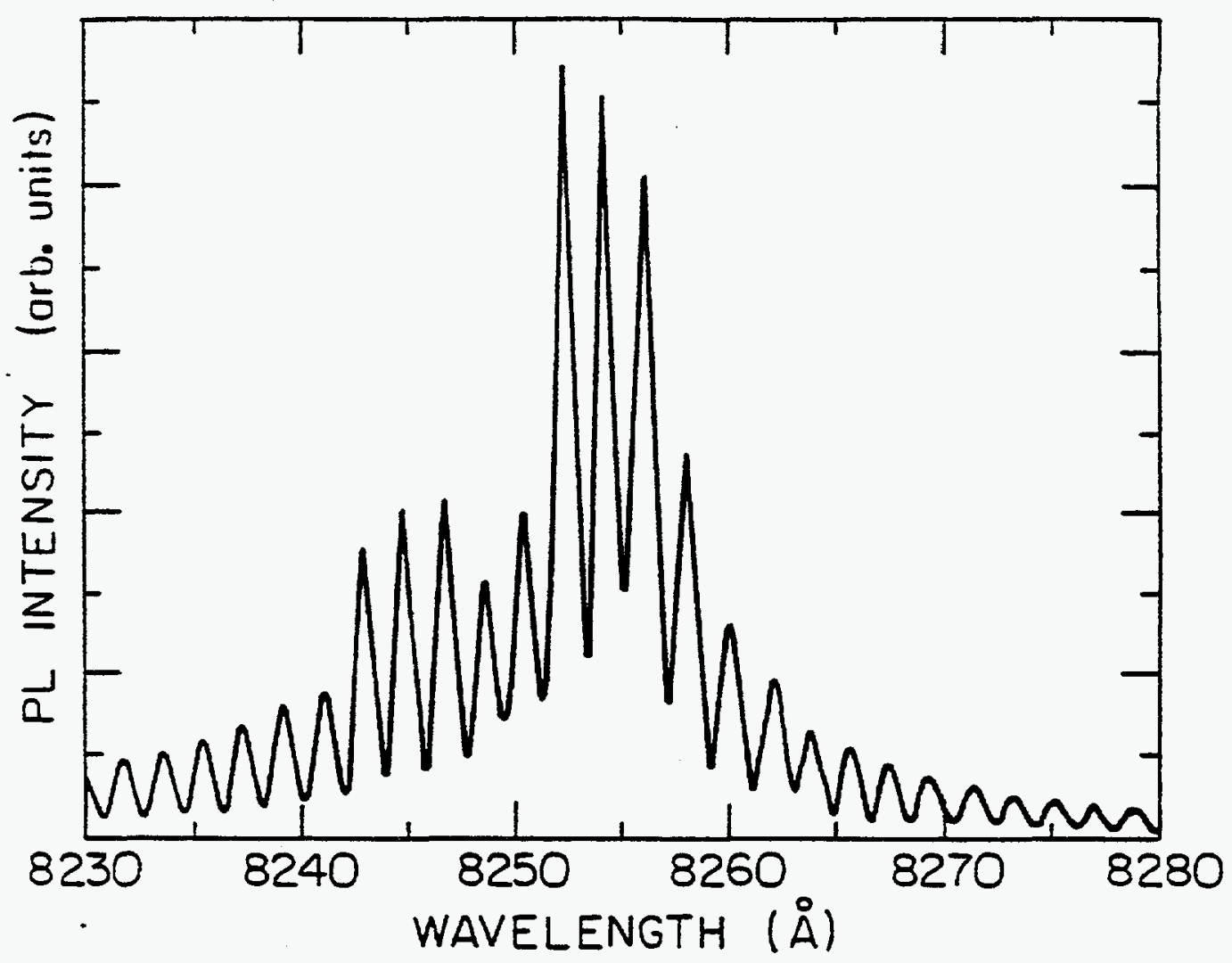

Figure 5.19

Mode spectrum of ALE SCH-QW injection laser showing longitudinal optical modes. 


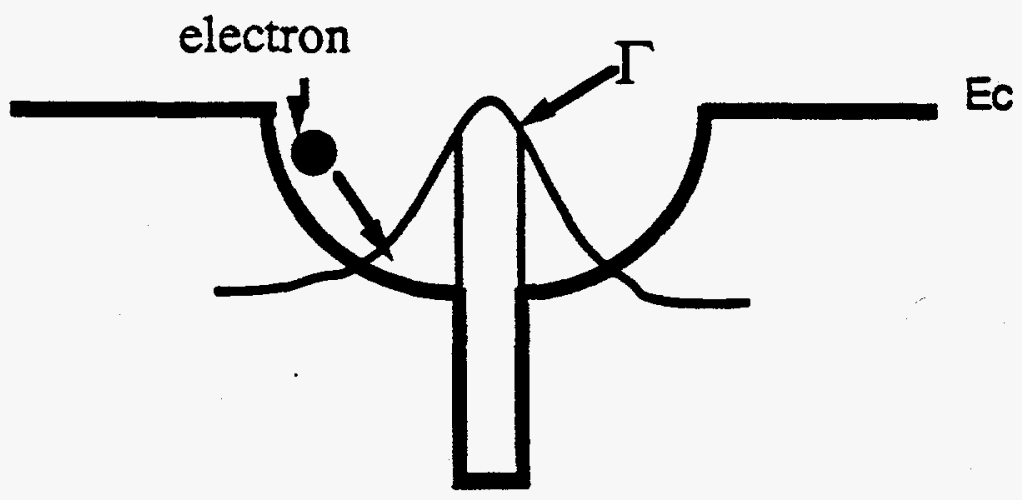

GRIN-SCH Structure

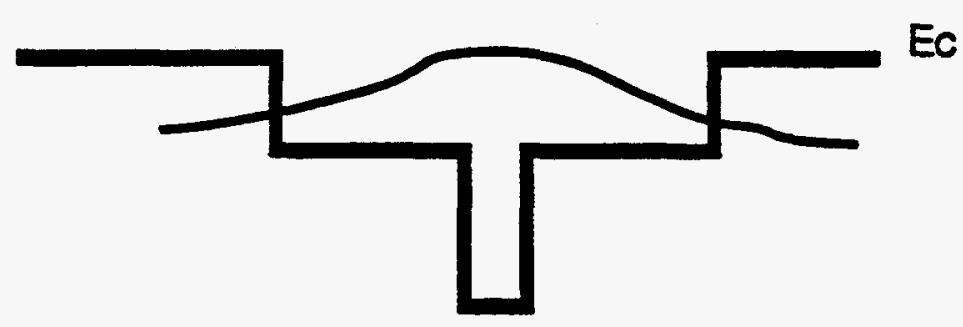

SCH Structure

Figure 5.20

Energy Band diagrams of GRIN-SCH and $\mathrm{SCH}$ laser structures Parabolic grading in GRIN-SCH creates pseudo-electric field and better optical confinement. 
167

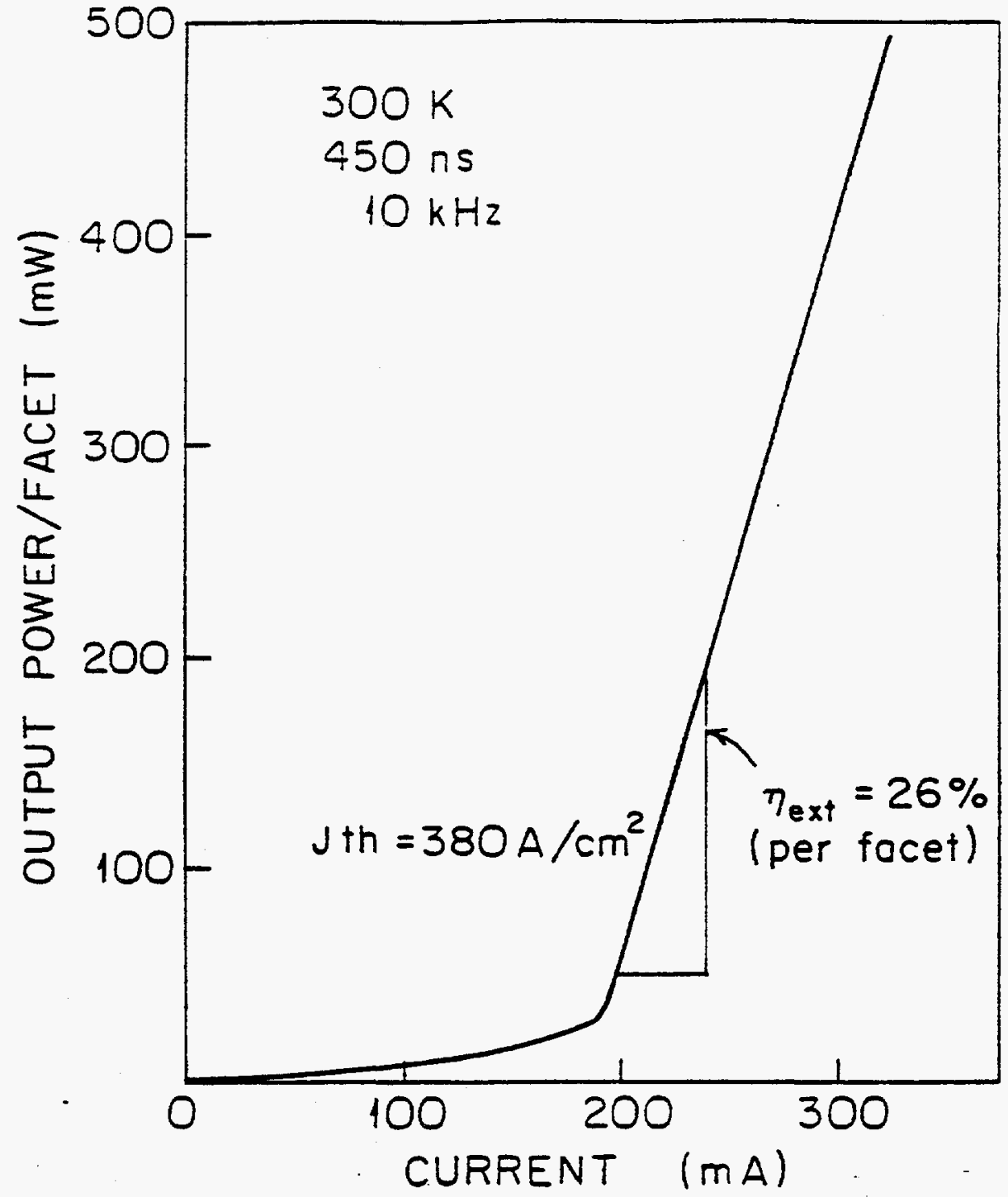

Figure 5.21

Luminescence intensity versus drive current for ALE $Q W$ injection laser 
168

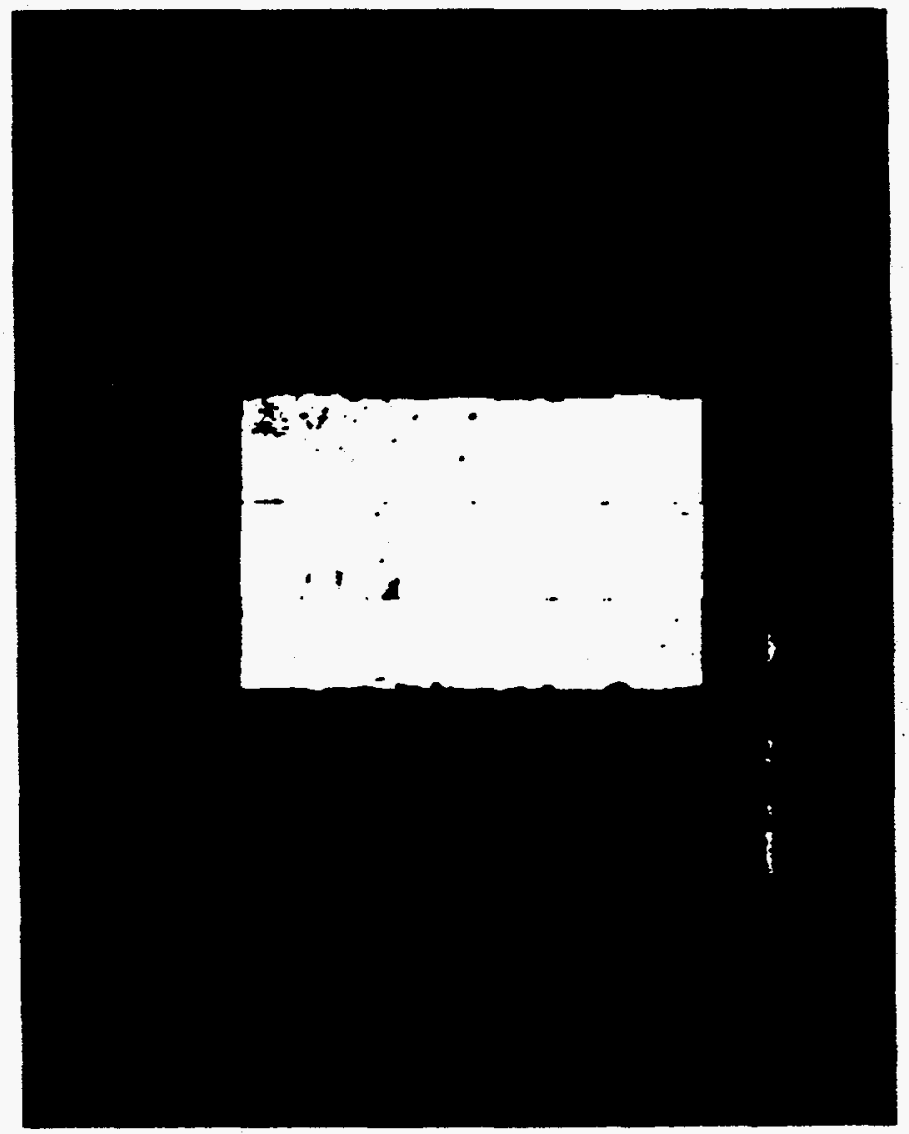

Figure 5.22

Top view of deposited $100 \mu \mathrm{m}$ wide $\mathrm{Cr}$-Au contact stripe on $\mathrm{QW}$ injection laser.(MAG=1000X) 


\subsection{Conclusions}

In conclusion, we have demonstrated for the first time operation of an injection laser grown by ALE. Low temperature PL of single quantum well (SQWs) grown by a hybridization of ALE and conventional MOCVD exhibit narrow linewidth intrinsic luminescence. Uniform layer thickness, high quality GaAs quantum well materials have also been demonstrated by utilizing the saturated monolayer growth process inherent to ALE. These results suggest that ALE will play an important role in the fabrication of heterojunction devices with critical layer thickness requirements.

\section{REFERENCES-CHAPTER 5}

1 Z. I. Alferov, V. M. Andreev, D. Z. Garbuzov, Y. V. Zhilyaev, E. P. Morozov, E. L. Portinoi, and V. G. Trofim, Sov. Phys. Semicond. 4, 1573 (1971) [Translated from Fiz. Tekh. Poluprovodn. 4, 1826 (1970)].

2 P. D. Dapkus, J. Crystal Growth 68, 345 (1984).

3 L. J. Mawst, M. E. Givens, C. A. Zmudzinski, M. A. Emanuel, and J. J. Coleman, IEEE J. Quantum Electron., QE-23, 696, (1987).

4 H. Okamato, Jap. J. Apll. Phys., 26, 315, (1987). 
5 R. Dingle, Festkorperprobleme XV, 21 (1975).

6 G. Bastard, Phys. Rev. B, 24, 5693 (1981).

7 H. C. Lee, PhD. Dissertation, University of Southern California (1987), p.130.

8 H. C. Lee, PhD. Dissertation, University of Southern California (1987), pp.124-127.

9 D. C. Bertolet, J. K. Hsu and K. M. Lau, J. Appl. Phys., 62,120 (1987).

10 J. Singh K. K. Bajaj and S. Caudhuri, Appl. Phys. Lett. 44, 805(1984).

11 D. M. Larsen, Phys. Rev. B8, 535 (1973).

12 C. F. Schaus, J. R. Shealy, L. F. Eastman, B. C. Cooman and C. B. Carter, J. Appl. Phys. 59, 678 (1986).

13 T. Fukunaga, K. L. I. Kobayashi, and H. Nakashima, Surface Sci., 174, 71 (1986).

14 J.Singh K. K. Bajaj and S. Caudhuri, Appl. Phys. Lett. 44, 805 (1984).

15 S. B. Ogale, A. Madhukar, F. Voillot, M. Thomsen, W. C. Tang, T. C. Lee, Y. Kim and P. Chen, Phys. Rev. B, 36,1662 (1987).

16 S. B. Ogale, A. Madhukar, F. Voillot, M. Thomsen, W. C. Tang, T. C. Lee, Y. Kim and P. Chen, Phys. Rev. B, 36, 1662 (1987). 
17 E. Colas, R. Bhat, B. Skromme, Paper D-8, International Symposium on GaAs and Related Compounds, Atlanta, GA (1988).

18 R. Z. Bachrach, Crystal Growth. 2nd ed., B.R. Pamplin, Pergamon Press, New York, (1980) p.260.

19 S. P. Denbaars, H. C. Lee, A. Hariz, P. D. Dapkus, N-3, Electronic Materials Conference, Santa Barbara, (1987).

20 M. Yoshida, H. Watanabe, and F. Uesugi, J. Electrochem. Soc. 137, 677 (1985).

21 T. Makimoto, Y. Yamauchi, and Y. Horikoshi, Paper D-7, International Symposium on GaAs and Related Compounds, Atlanta, GA (1988).

22 L. J. Mawst, M. E. Givens, C. A. Zmudzinski, M. A. Emanuel, and J. J. Coleman, IEEE J. Quantum Electron., QE-23, 696, (1987).

23 N. Y. Holonyak, R. M. Kolbas, R. D. Dupuis, P. D. Dapkus, IEEE J. Quantum Electronics, QE-16, 170 (1980).

24 W. T. Tsang, Appl. Phys. Lett. 40, 217 (1982).

25 D. Kasemet, C. Hong, N. B. Patel, and P. D. Dapkus, IEEE J. of Quantum Elec., QE-19, 1025 (1983).

26 H. C. Casey, Jr and M. G. Panish, Heterostructure Lasers, (New York, Academic Press, 1978) p.167.

27 P. D. Dapkus, Class Notes, EE-599, Heterostructure Materials and Devices, University of Southern California, (1986).

28 W. H. Tsang, Appl. Phys. Lett., 40, 217 (1982). 
29 H. C. Casey, Jr and M. G. Panish, Heterostructure Lasers, (New York, Academic Press, 1978) p.176.

30 S. P. DenBaars, C. A. Beyler, A. Hariz, and P. D. Dapkus, Appl. Phys. Lett., 51, 1530 (1987). 


\section{CHAPTER 6}

\section{LASER ASSISTED ATOMIC LAYER EPITAXY}

\subsection{Introduction}

Laser induced chemical vapor deposition (LCVD) has been identified as an attractive alternative for the fabrication of microelectronic devices. Low temperature processing, selective area deposition, and wavelength selective photochemistry are some of the unique aspects offered by LCVD. Because of the spatial and temporal selectivity of depositions available through the use of lasers, new methods of semiconductor processing are anticipated. LCVD of conducting metallic films has recently resulted in excellent metallic films. ${ }^{1}$ However, LCVD of semiconductors have been plagued by polycrystalline deposits with high carbon content.

Laser assisted ALE (LALE) has recently been discovered to allow the selective growth of single crystal GaAs. ${ }^{2}$ The deposited films were found to be p-type with background doping of the $5 \times 1017 / \mathrm{cm}^{3}$. In this method, modulation of both a CW Argon ion laser and the gas stream is performed. The laser irradiates the surface for 1 second during the gallium exposure to achieve selective decomposition of the TMGa adsorbate on the As atoms. Perfect monolayer saturation of the growth rate is observed over a wide range of trimethylgallium (TMGa) fluxes and temperatures. The selective deposited structure was typically a $0.5 \mathrm{~mm}$ by $1 \mathrm{~mm}$ elliptical deposit. Only deposition on GaAs surfaces has been studied to date. 
We have elected to study the fast writing potential of LALE on AlGaAs surfaces with the goal of incorporating the GaAs in heterostructure devices. Selective area epitaxy by laser assisted ALE has great potential for the integration of opto-electronic and electronic devices. Optimization of LALE on AlGaAs surfaces would allow the in situ deposition of heterostructure devices with different epitaxial structures. The manufacturing benefits of a "laser writing" process which affords the fabrication of various device structures in a single run are enormous. In this study we demonstrate the "fast writing" potential of LALE for depositing small dimension structures .

The approach we have taken is to employ conventional MOCVD reactants to allow for the hybridization of ALE and conventional MOCVD in a novel four chamber reactor. The four chamber reactor allows spatial separation of the column III and column V precursors, thus allowing one monolayer per rotation in as little as 2 seconds. In contrast to the work of Doi et al., 2 we have employed a scanned laser beam with spot sizes as small as $30 \mu \mathrm{m}$ to selective deposit GaAs stripes on Al0.3Ga0.7 As. ${ }^{3}$ We observe monolayer saturation in the growth rate under a wide range of growth conditions. The LALE deposits exhibit smooth mirror like morphology with a flat-topped profiles of thickness, indicative of a monoatomic growth mechanism. 


\subsection{Laser CVD Theory}

\subsubsection{Photocatalytic Deposition}

Laser CVD is based on the interaction of laser light with molecules in the gas phase and/or on the substrate surface. The laser CVD approach can be divided into three categories of reactions mechanisms: photocatalytic, photolytic, and pyrolytic. Photocatalytic reactions are defined as an increase in a chemical reaction rate produced by photogenerated carriers in a solid where the solid remains unchanged. 4 A general expression of a photocatalyzed surface reaction in a semiconductor is:

$$
\begin{gathered}
\text { S.C. (Eg) }+\mathrm{h} v(\mathrm{~h} v>E g) \Rightarrow \mathrm{e}^{-}+\mathrm{h}^{+} \\
\mathrm{e}^{-}+\mathrm{X} \Rightarrow \mathrm{X}^{-} \\
\mathrm{h}^{+}+\mathrm{M} \Rightarrow \mathrm{M}^{+} \\
\mathrm{X}^{-}+\mathrm{M}^{+} \Rightarrow \mathrm{XM}
\end{gathered}
$$

A few of the photocatalytic reactions which are known to occur upon the illumination of a semiconductor are the dehydrogenation of isopropyl alcohol, and the splitting of water molecules. 5 Because the wavelength of the Argon ion laser utilized in the LALE experiments is larger than the bandgap of GaAs, a surface photocatalytic process is a likely growth mechanism. 
The deposition rate for a surface photo-reaction will be directly proportional to the power density. Assume the substrate is illuminated with a gaussian laser beam of spot size $w_{0}$. For a laser of power $P_{L}$ the laser power density at a point $r_{0}$ from beam center is given by:

$$
P\left(r_{o}, w_{o}\right)=\left(\frac{2 P_{L}}{\pi w_{0}^{2}}\right) \exp \left(-\frac{2 r_{o}^{2}}{w_{o}^{2}}\right)
$$

The number of adsorbed molecules per $\mathrm{cm}^{2}$, will be equal to the surface coverage " $\Theta$," multiplied by the surface sites per unit area $N_{\mathbf{s}}$ :

$$
\mathrm{N}_{\mathrm{a}}=\theta \mathrm{N}_{\mathrm{s}}
$$

The photon flux per unit surface area will be the power density divided by the photon energy:

$$
J_{\Phi}=\frac{1}{h v}\left(\frac{2 P_{L}}{\pi w_{0}^{2}}\right) \exp \left(-\frac{2 r_{0}^{2}}{w_{0}^{2}}\right)
$$

The rate of deposition at a specific point will include the quantum efficiency $\eta$, absorption cross-section $\sigma_{a}$ of the film, the number of adsorbed molecules, and the photon flux:

$$
\mathrm{R}_{\mathrm{sur}}=\mathrm{J}_{\Phi}\left(\eta \theta \mathrm{N}_{\mathrm{s}} \sigma_{\mathrm{a}}\right)
$$

Substituting in above equations the rate of deposition in molecules $/ \mathrm{cm}^{2} \cdot \mathrm{sec}$ is: 


$$
R_{\text {sur }}=\frac{\eta \theta N_{s} \sigma_{a}}{h v}\left(\frac{2 P_{L}}{\pi w_{o}^{2}}\right) \exp \left(-\frac{2 r_{0}^{2}}{w_{0}^{2}}\right)
$$

This expression can be further simplified by noting the fastest rate of deposition will be at the center of the beam $\left(r_{0}=0\right)$. Dividing by the surface site density will yield the number of monolayers deposited per second:

$$
\mathbf{R}_{\mathrm{sur}}^{\max }=\frac{\eta \theta\left(2 \sigma_{\mathrm{a}} \mathrm{P}_{\mathrm{L}}\right)}{\left(h v \pi \mathrm{w}_{0}^{2}\right)}
$$

\subsubsection{Pyrolytic Deposition}

Pyrolytic deposition is caused by laser radiation absorbed in the substrate which leads to a local increase in temperature at the surface. Molecules either on the surface or in the nearby gas phase dissociate by pyrolysis. The reaction rate is determined principally by the maximum temperature rise $\Delta T$. For a stationary Gaussian beam of power $P_{L}$ the maximum temperature rise is given by: 6

$$
\Delta \mathrm{T}_{\max }=\frac{\mathrm{P}_{\mathrm{L}}(1-\mathrm{R})}{\left(2 \sqrt{\pi} \mathrm{w}_{\mathrm{o}} \mathrm{K}\right)}
$$

where $\mathrm{R}$ is the surface reflectivity, $\mathrm{K}$ the thermal conductivity, and $\mathrm{w}_{\mathrm{o}}$ the beam radius at $1 / \mathrm{e}^{2}$ intensity. For the LALE work of Doi et al. ${ }^{2}$ this 
equation yields an estimated temperature rise of only $40^{\circ} \mathrm{C}$ for the stationary irradiation case. If we operate at a low temperatures, where kinetics of surface reactions dominant ( $\mathrm{T}<550^{\circ} \mathrm{C}$ for $\mathrm{MOCVD}$ ), then growth is not controlled by the flux of reactant to the surface and we can expresses the growth rate as a temperature activated process:

$$
G_{r}=A \exp \left(-\frac{E_{a}}{k T}\right)
$$

where $E_{a}$ is the activation energy of the process, $k$ is Boltzmann's constant, and $\mathrm{A}$ is a proportionality constant. Therefore we should see an exponential increase in the growth rate with laser power if pyrolytic mechanisms dominate.

\subsubsection{Photolytic Deposition}

Photolytic deposition consists of direct dissociation of one of the molecular bonds of a molecule by absorption of one or more photons. The photodissociation of molecules is typically defined by its dissociation spectrum: 7

$$
\sigma_{D}(\lambda)=\eta \sigma_{A}(\lambda)
$$

where $\sigma_{D}$ is the photodissociation cross-section, $\sigma_{\mathrm{A}}$ is the total absorption cross, and $\eta$ is the quantum efficiency of the photodissociation process. For a single photon dissociation process, as shown in figure $6.1, \eta$ is essentially unity, whereas for a multi-photon dissociation process $\eta$ can be 
quite low. For a gas phase photolytic decomposition process molecules above the substrate will be photodissociated and will contribute to film growth. In this case we can approximate the photolytically decomposed species as a cylindrical column with the same waist as the laser beam $\mathrm{w}_{0}$. For gas phase photodecomposition the flux impinging on the surface the beam center will be: 6

$$
J_{g a s}=\frac{\eta\left(N_{g} \sigma_{g} P_{L} w_{o}\right)}{\left(2 h v \pi w_{0}^{2}\right)}
$$

where, $\mathrm{N}_{\mathrm{g}}$ is the molecular gas phase density, $\mathrm{P}_{\mathrm{L}}$ the laser power, $w_{0}$ the beam radius, and $\sigma_{\mathrm{g}}$ the absorption coefficient. The rate of deposition on the surface in monolayers per second will be the flux times the photodissociated molecule sticking coefficient $\beta$, divided by the surface site density:

$$
R_{g a s}=\frac{\beta \eta\left(N_{g} \sigma_{g} P_{L} w_{o}\right)}{N_{s}\left(2 h v \pi w_{0}^{2}\right)}
$$

From the above equation 6.11 we can deduce the growth will be proportional to the inverse of the spot size times the laser power.

$$
R_{\text {gas }} \sim \frac{P_{L}}{\left(w_{0}\right)}
$$


whereas if surface phase deposition dominates, it can be seen from equation 6.6 that growth will be directly proportional to the laser power density:

$$
R_{\text {sur }} \sim \frac{P_{L}}{\left(w_{0}\right)^{2}}
$$

Therefore, we will be able to determine if the gas or surface phase photodecomposition process is the dominant mechanism by simply varying the spot size of the laser beam. 


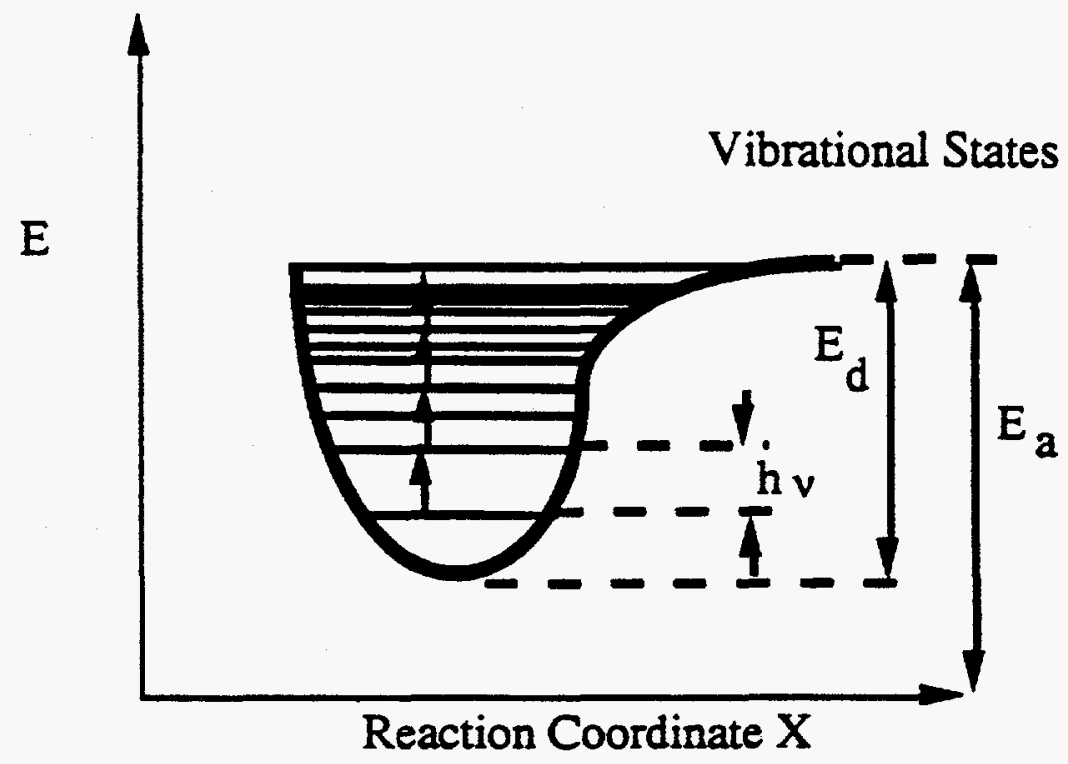

(A)

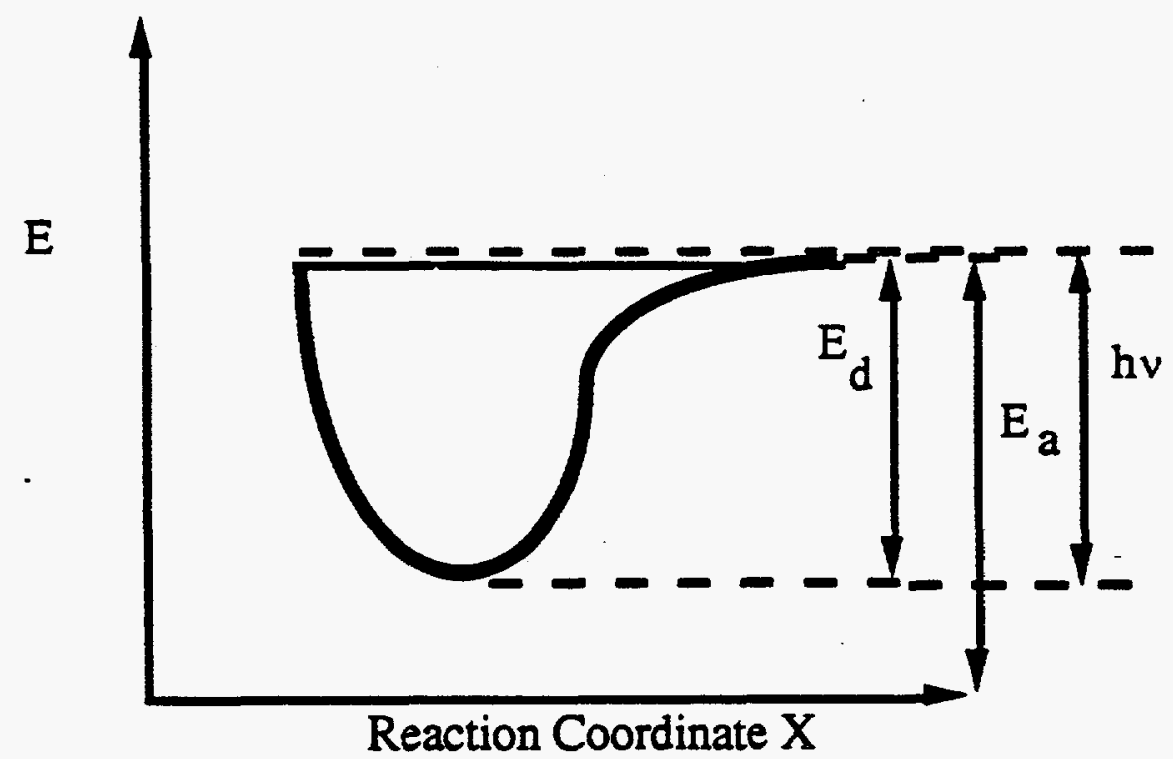

(B)

Figure 6.1

Photolytic deposition is achieved by excitation of molecular bonds by either (A) Multi-photon absorption, or (B) a single high energy photon

( $E_{d}$ is the dissociation energy, hv the photon energy) 


\subsection{EXPERIMENTAL}

LALE has been accomplished in this work using conventional MOCVD precursors (TMGa and $\mathrm{AsH}_{3}$ ) and conventional vent/run MOCVD reactors altered to allow alternate exposure of the substrate to these precursors. In the new reactor design reactant exposures are séparated spatially by utilizing a four chamber vertical reactor in which the substrate is rotated in continuous reactant flows. Figure 6.2 shows a schematic of the experimental setup and new reactor design. RF induction was utilized in this reactor design to heat the susceptor. LALE experiments were performed by directing a focused laser onto the substrate during the TMGa exposure. In this way the laser was effectively scanned across the surface forming an epitaxial stripe. The reactor was equipped with a variable speed rotation control so that exposures to TMGa and $\mathrm{AsH}_{3}$ could be separately optimized at a given temperature. The sample was then rotated through a high gas velocity hydrogen purge chamber which effectively "flushed" excess TMGa off the surface. The third chamber exposed the substrate to $\mathrm{AsH}_{3}$ which reacted with the gallium adsorbate to form one monolayer before proceeding through another hydrogen chamber. A complete rotation corresponding to one monolayer could be accomplished in as fast as 2 seconds. Typical rotation speeds were 0.11 revolutions/sec.

Under appropriate conditions one monolayer of a $\mathrm{Ga}$ adsorbate was formed uniformly only in the region of laser excitation for LALE during 
the TMGa exposure. This adsorbate which we believe to be $\mathrm{GaCH}_{3}$ subsequently reacts with $\mathrm{AsH}_{3}$ to form $\mathrm{GaAs}$. It has been found beneficial to utilized UV illumination during the $\mathrm{AsH}_{3}$ exposure to improve crystalline morphology. The high energy UV photons photolytically decompose the $\mathrm{AsH}_{3}$ which increase the surface reaction rate with the $\mathrm{Ga}$ adsorbate. 8 In this reactor design conventional MOCVD could be performed by raising the substrate temperature and injecting the reactants simultaneously into the same chamber. This gives the ability to overgrow the structures with high quality AlGaAs confining regions 


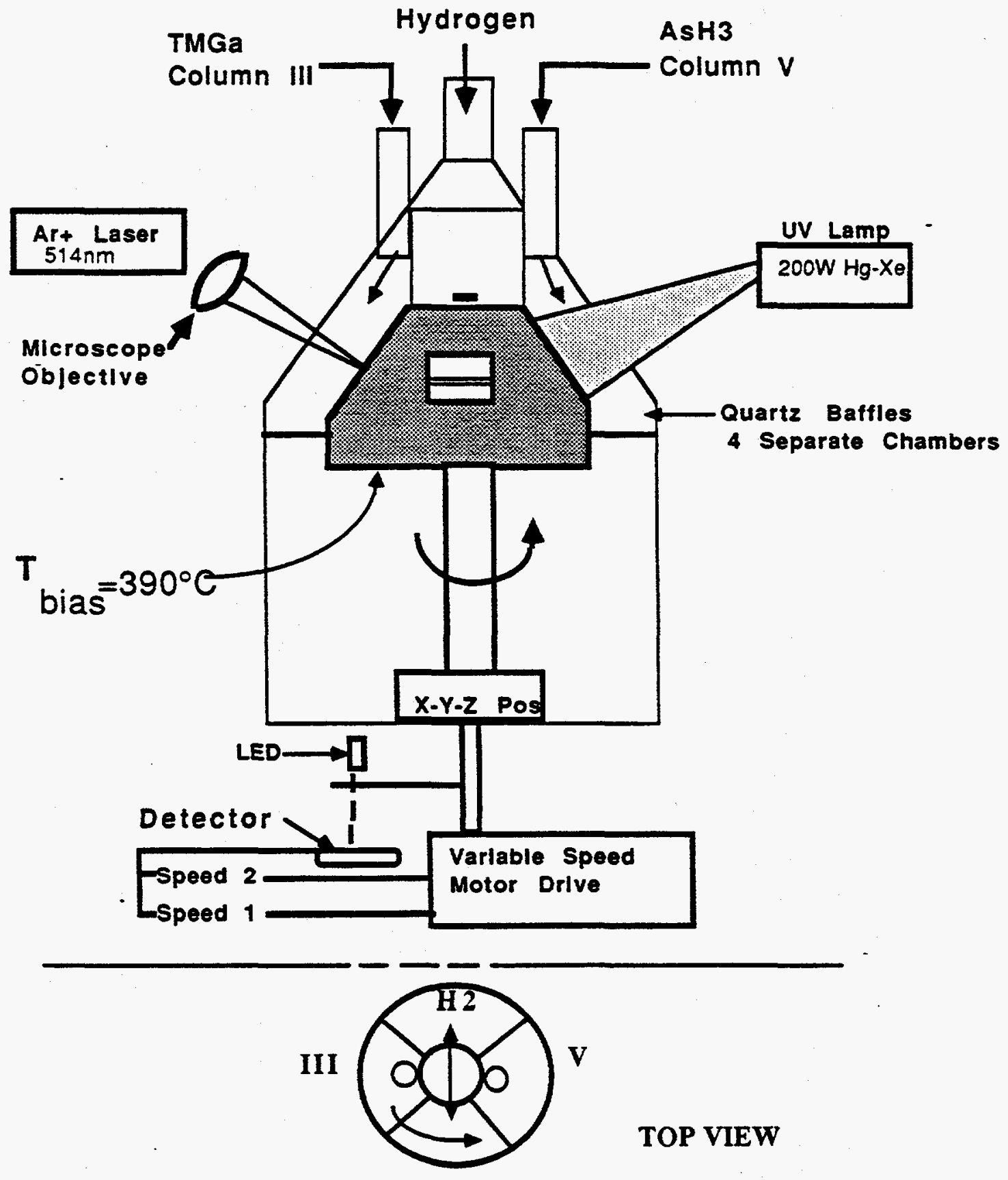

Figure 6.2

Schematic of four chamber reactor used in laser assisted ALE experiments. Note that the column III and column V reactants are separated spatially by two hydrogen purge channels and quartz baffles. 


\subsection{RESULTS \& DISCUSSION}

Using the four chamber reactor described previously we have conducted a series of experiments to characterize LALE growth using Argon ion laser irradiation during the TMGa exposure cycle. By scanning the laser spot, $40 \mu \mathrm{m}$ in diameter, at speeds up to $4000 \mu \mathrm{m} / \mathrm{sec}$ we have selectively deposited single crystal GaAs stripes on both $\mathrm{Al} 0.3 \mathrm{Ga0} .7 \mathrm{As}$ and GaAs surfaces. As shown in figure 6.3, perfect monolayer saturated growth over an order of magnitude in TMGa pressures is demonstrated. Thickness measurements were made with a surface profilometer accurate to within $\pm 50 \AA$ and correlated to the number of rotations. The typical flat-top deposit profile is illustrated in figure 6.4. The fact that the deposit exhibits a plateau shape, instead of the Gaussian profile of the laser beam intensity, demonstrates the monoatomic nature of the surface photocatalytic mechanism operative in this process. The flat-top nature of the deposit has also been observed for larger geometry deposits. 


\subsection{RESULTS \& DISCUSSION}

Using the four chamber reactor described previously we have conducted a series of experiments to charaterize LALE growth using an Argon ion laser irradiation during the TMGa exposure cycle. By scanning the laser spot, $40 \mu \mathrm{m}$ in diameter, at speeds up to $4000 \mu \mathrm{m} / \mathrm{sec}$ we have selectively deposited single crystal GaAs stripes on both $\mathrm{Al}_{0.3} \mathrm{Ga}_{0.7} \mathrm{As}$ and $\mathrm{GaAs}$ surfaces. As shown in figure 6.3, perfect monolayer saturated growth over an order of magnitude in TMGa pressures is demonstrated. Thickness measurements were made with a surface profilometer accurate to within $\pm 50 \AA$ and correlated to the number of rotations. The typical flat-top deposit profile is illustrated in figure 6.4. The fact that the deposit exhibits a plateau shape, instead of the Gaussian profile of the laser beam intensity, demonstrates the monoatomic nature of the surface photocatalytic mechanism operative in this process. The flat-top nature of the deposit has also been observed for larger geometry deposits. 


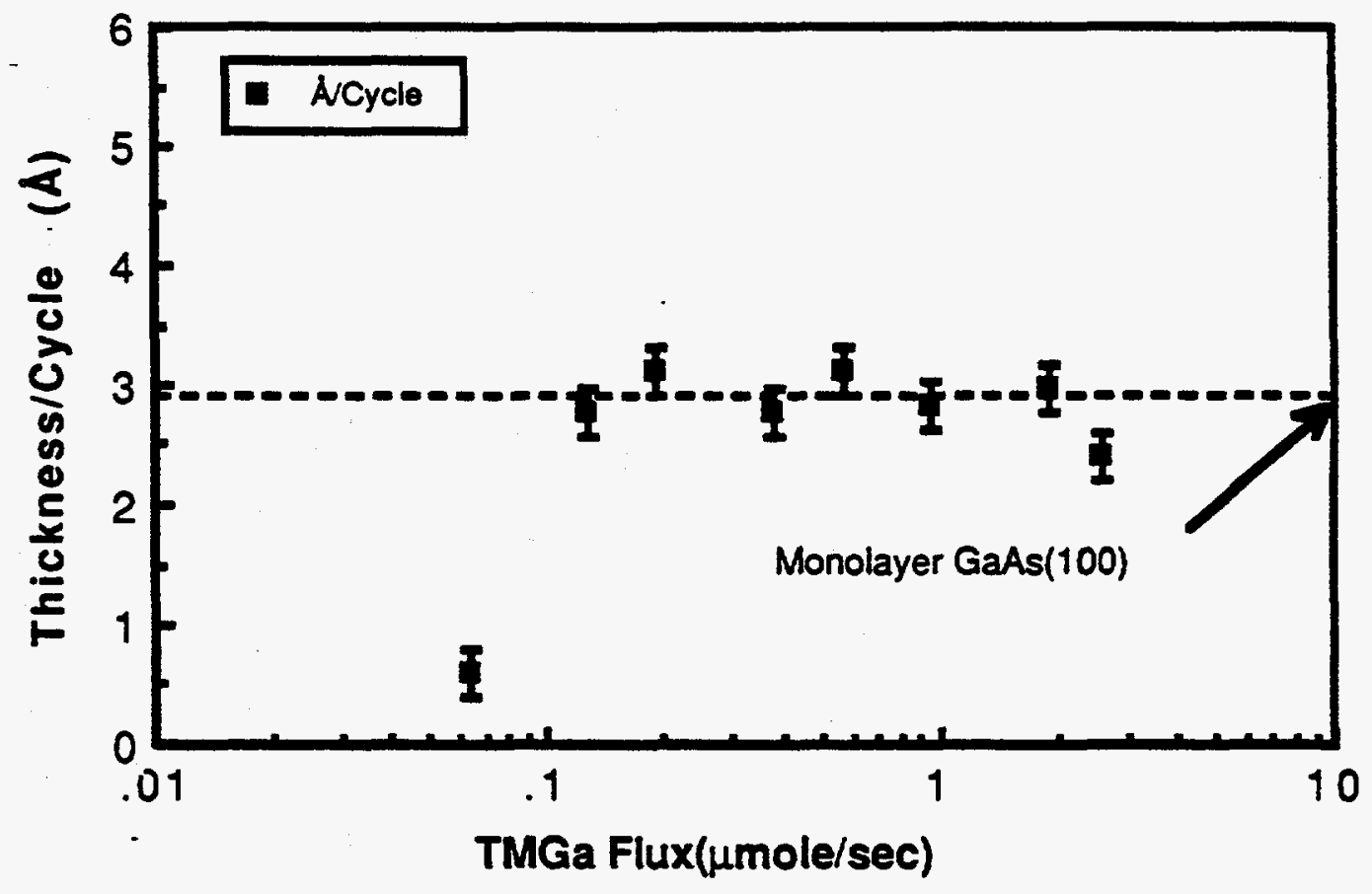

Figure 6.3.

Laser assisted ALE exhibits self-limiting monolayer growth over a wide range of TMGa fluxes. 


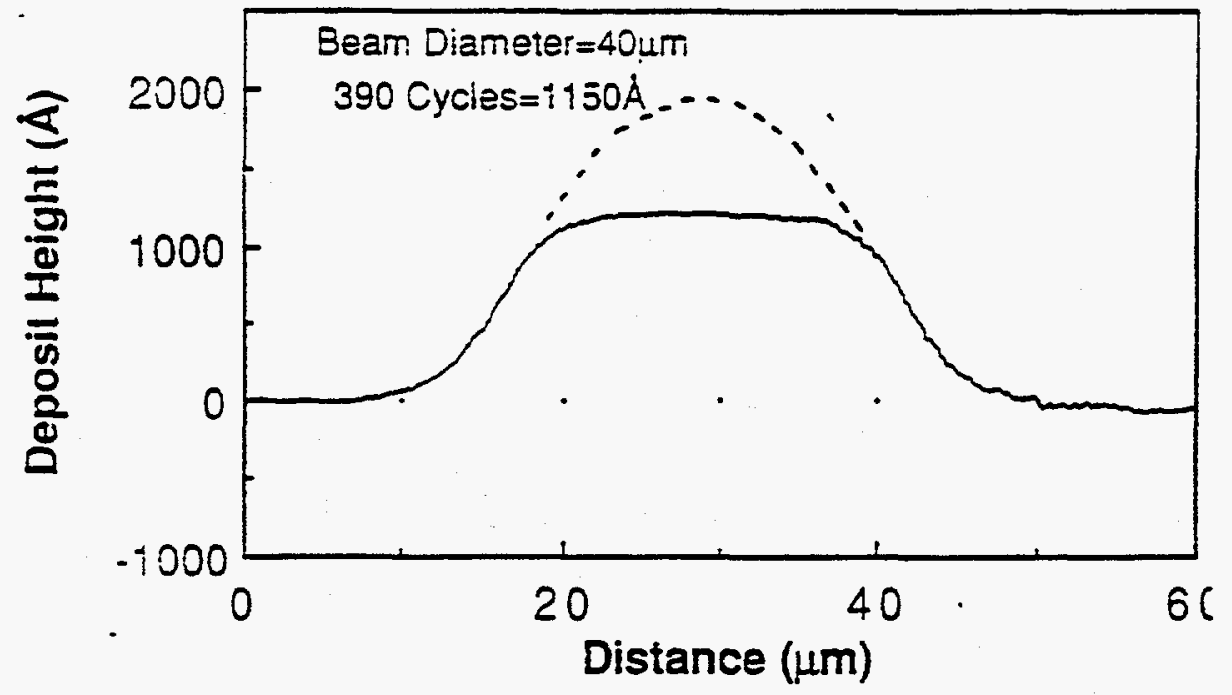

Figure 6.4.

LALE deposits exhibit flat-top profile indicative of mono-atomic growth mechanism. 


\section{Laser ALE Deposit}

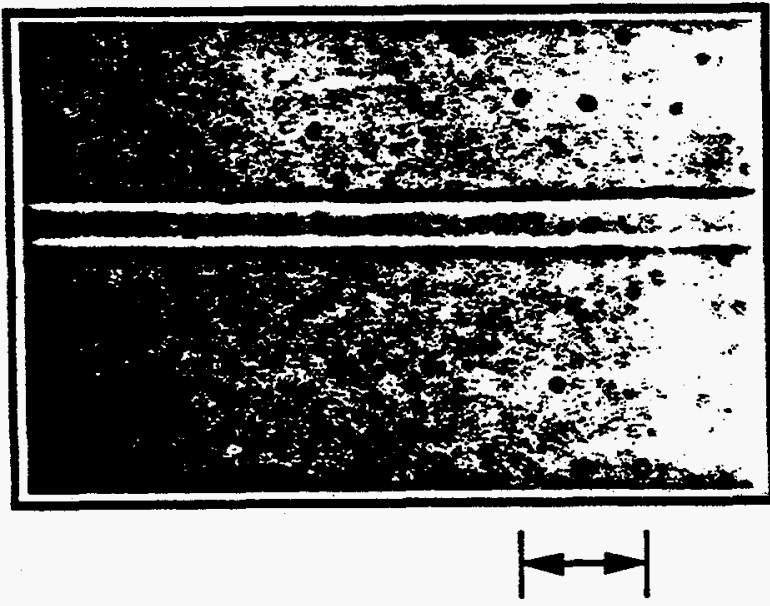

$100 \mu \mathrm{m}$

Figure 6.5

Photomicrograph of LALE GaAs stripe deposits exhibits smooth morphology of flat top stripes.(MAG $=100 \mathrm{X}$ ) 
Figure 6.5 shows a photomicrograph which illustrates the smooth surface morphology of the deposited stripes. Note the flat-top nature of the deposit is also visible. In order to evaluate the potential of LALE for heterostructure devices, we have overgrown the deposited stripes with AlGaAs. This was performed by conventional MOCVD in the same reactor by heating the sample up to $750^{\circ} \mathrm{C}$, and then injecting all reactants simultaneously into the hydrogen purge chamber. Figure 6.6 shows the low temperature PL obtained from a series of closely spaced LALE GaAs stripes which have been overgrown with $\mathrm{Al}_{0.3 \mathrm{Ga}} \mathrm{G}_{0.7} \mathrm{As}$. Donor to acceptor luminescence dominates the spectra. The measured energy difference of the peak from the band edge identify carbon as the dominant impurity in the sample. We believe this to be due to the low bias temperature used in these experiments $\left(T=390^{\circ} \mathrm{C}\right)$. The lower temperature would decrease the surface exchange reaction rate between the gallium adsorbate and the arsine 
As a means of reducing the carbon content of the films, UV irradiation of the wafer during the arsine exposure step was added High energy UV photons produce photolytic cracking of the arsine and increase the rate of surface reactions. Surface morphology of the deposits was dramatically improved upon the addition of the Hg-Xe UV lamp. The $200 \mathrm{~W} \mathrm{Hg}-\mathrm{Xe}$ lamp is housed in an metallic elliptical mirror assembly which produces a highly concentrated optical beam. The power denisity of deep UV irradiation is estimated to be $10 \mathrm{~W} / \mathrm{cm}^{2}$ at the GaAs surface. In addition, we have observed that UV irradiation has improved the PL linewidth of QWs grown by thermal ALE. This is as shown in figure 6.7. Further analysis of UV exposure on the deposits is necessary. Another means of reducing the carbon content in the LALE process would be the addition of thermal pre-cracking of the arsine. Use of an infrared spot heater to locally heat the susceptor upstream of the wafer to above $550^{\circ} \mathrm{C}$ would generate As2, As4, and arsenic hydride radical species which might play a role in reducing carbon incorporation. 


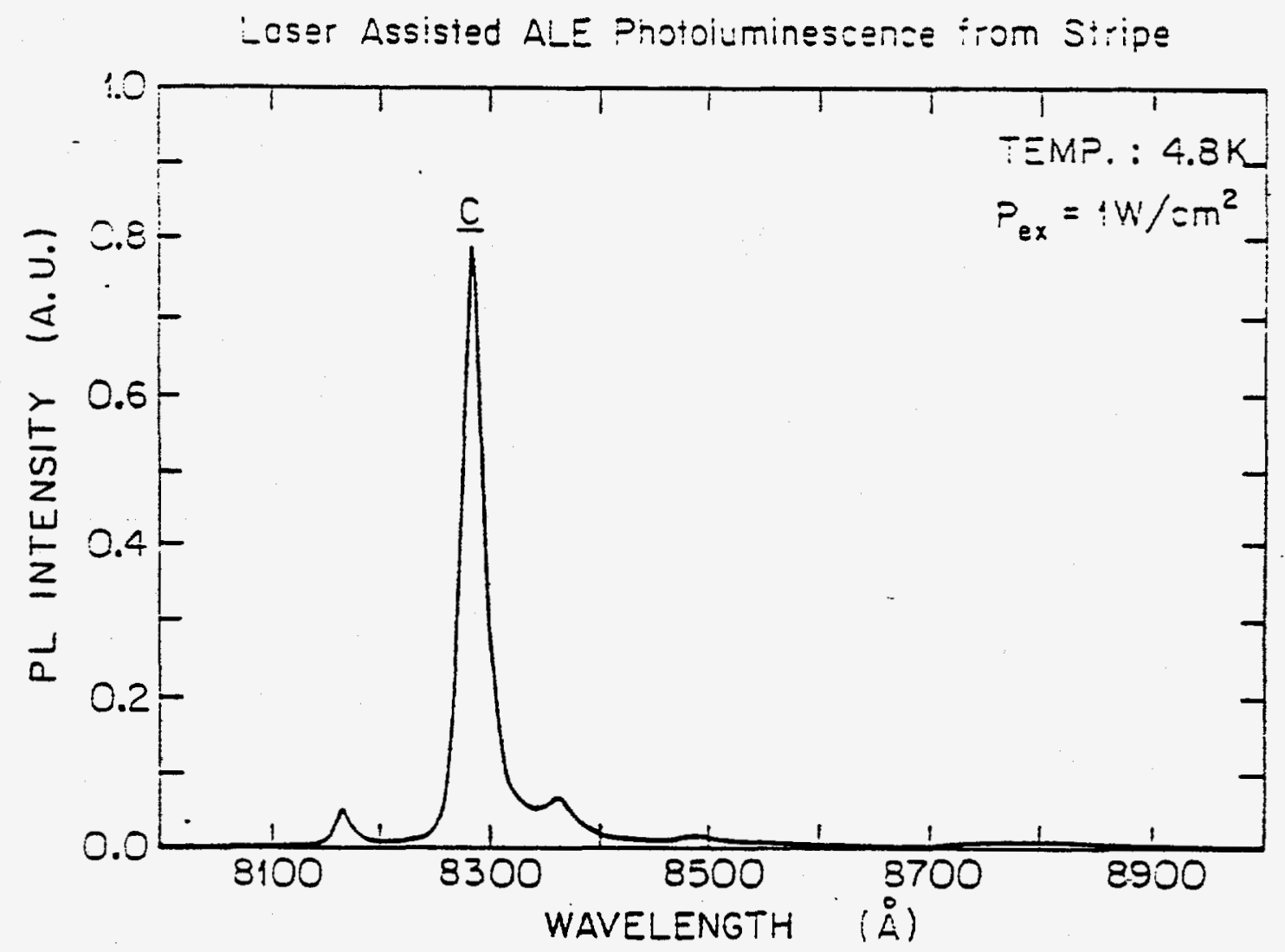

Figure 6.6

Low temperature PL from buried LALE GaAs stripes displays impurity related luminescence 


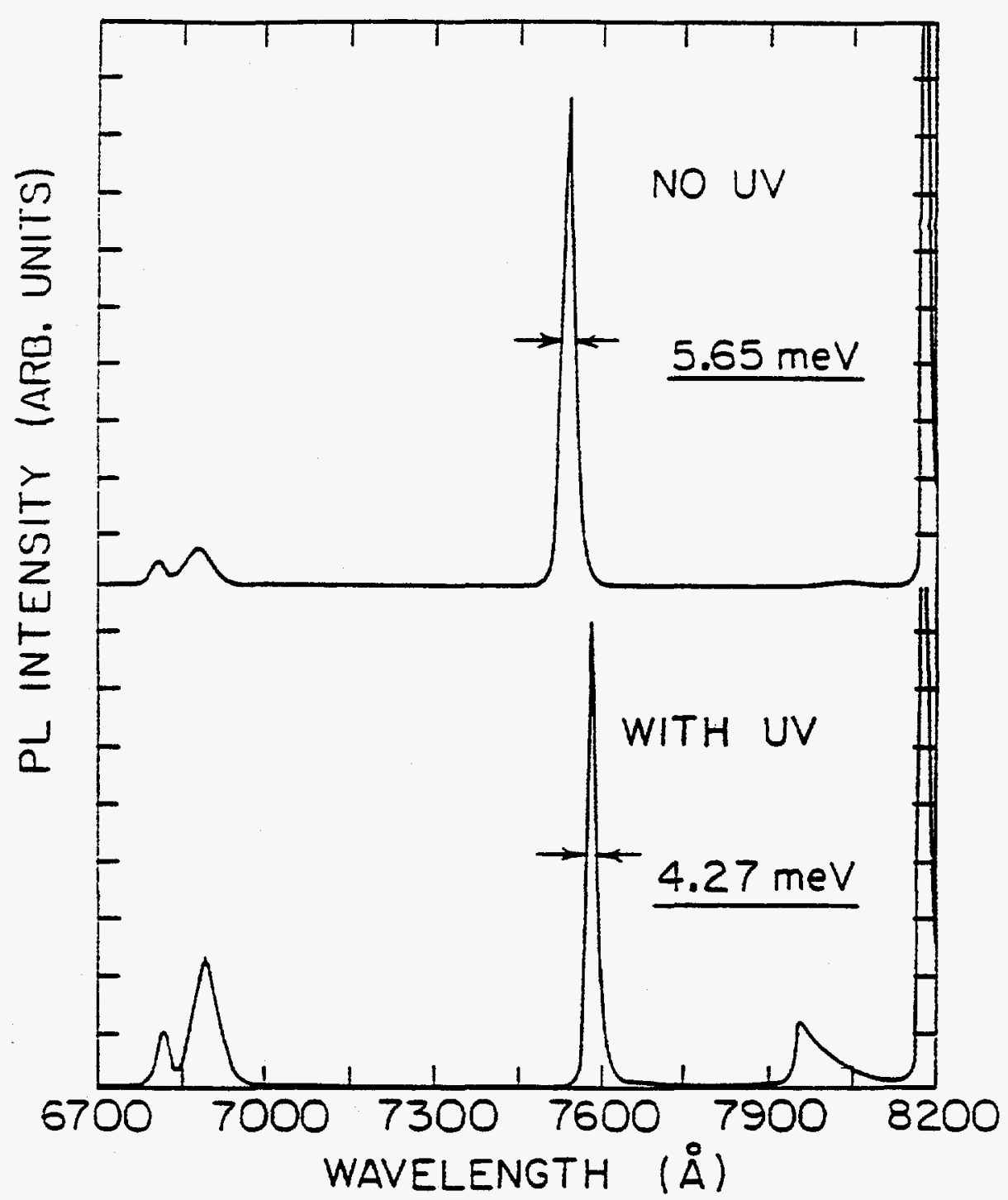

Figure 6.7

UV illumination is observed to decrease the PL linewidth of thermally deposited ALE QWs. 


\subsection{Proposed LALE Mechanism}

The Argon ion laser irradiation locally stimulates the growth by a surface photo-reaction mechanisms. We propose a two mechanism LALE model that takes into account a self-limiting surface photocatalytic mechanism and surface heating. Growth saturation over a range of laser powers, as shown in Figure 6.8, argues against a purely thermally effect. This is also supported by the observation that at these writing speeds the growth saturates over a $50^{\circ} \mathrm{C}$ range of temperatures. But this data also shows that above critical powers the growth rate increases due to the pyrolytic nature of the laser irradiation. Figure 6.9 provides a comparison of LALE growth data to thermal ALE which suggests different growth kinetics are involved in the two techniques. Thermal ALE requires partial gas or surface phase cracking to yield a $\mathrm{Ga}$ adsorbate, while LALE appears to generate selective decomposition by a surface photochemical reaction alone. However, some local heating is necessary to effect single crystal growth since the growth rate starts to drop off with decreasing bias temperature. The deposition rate for LALE is described by modifying the first order adsorption model described in thermally driven ALE to account for the photocatalytic and pyrolytic nature of the laser irradiation:

$$
\frac{d \theta}{d t}=k_{\text {laser }}^{P_{L}}(1-\theta)+k_{\text {ads }}^{\Delta T}(1-\theta)+k_{\text {des }}^{\Delta T}(\theta)+k_{G a}^{\Delta T}
$$


Where $k_{\text {laser }}$ is the rate constant for the photocatalytic effect of the laser beam which is directly proportional to the laser power:

$$
\underset{\text { laser }}{P_{L}}=\frac{\eta\left(2 \sigma_{a} P_{L}\right)}{\left(h v \pi w_{o}^{2}\right)}
$$

The thermal effect of the laser beam is taking into account by calculating the temperature rise $\Delta T_{\text {laser }}$ caused by the scanned beam and substituting this into the rate constant for thermal ALE. Equation 4.7 then becomes:

$$
k_{\text {ads }}^{\Delta T}=\frac{\delta\left(C_{\text {TMGa }}\right)\left(1 \times 10^{18} \exp \left(-\frac{60 \mathrm{kcal}}{R\left(T_{\text {bias }}+\Delta T_{\text {laser }}\right)}\right)\right)}{N_{s}}
$$

Where $T_{\text {bias }}$ is the temperature at which the substrate held without laser irradiation in the LALE experiments $\left(390^{\circ} \mathrm{C}\right), \mathrm{R}$ is the gas constant, and $\mathrm{C}_{\mathrm{TMGa}}$ the concentration of the TMGa in the gas. By similarly altering the thermal rate constant listed in Table 4.2 we can generate a deposition model. The rate of growth caused by the photocatalytic effect can be expressed by an enhancement factor in comparison to the thermal rate constant. This model uses a photocatalytic growth enhancement value of 200, which was observed in cw LALE results. 9 


$$
\left(\begin{array}{c}
\mathrm{k}_{\mathrm{L}} \\
\frac{\mathrm{k}_{\text {laser }}}{\mathrm{T}_{\mathrm{b}}}
\end{array}\right)=200
$$

To account for the scanning of the laser beam, the model using the effective reaction time " $\Delta \tau$ " which is the time of the laser on the deposited spot area. For our scanned LALE technique this is estimated to be the spot size divided by the linear velocity $\left(40 \mu \mathrm{m} / 4000 \mu \mathrm{m} / \mathrm{sec}=1 \times 10^{-2} \mathrm{sec}\right.$ $=\Delta \tau)$. Figure 6.10 shows the predicted growth rate for laser ALE. Note that the experimentally observed independence of growth rate over a range of temperatures is predicted by the computer generated model.

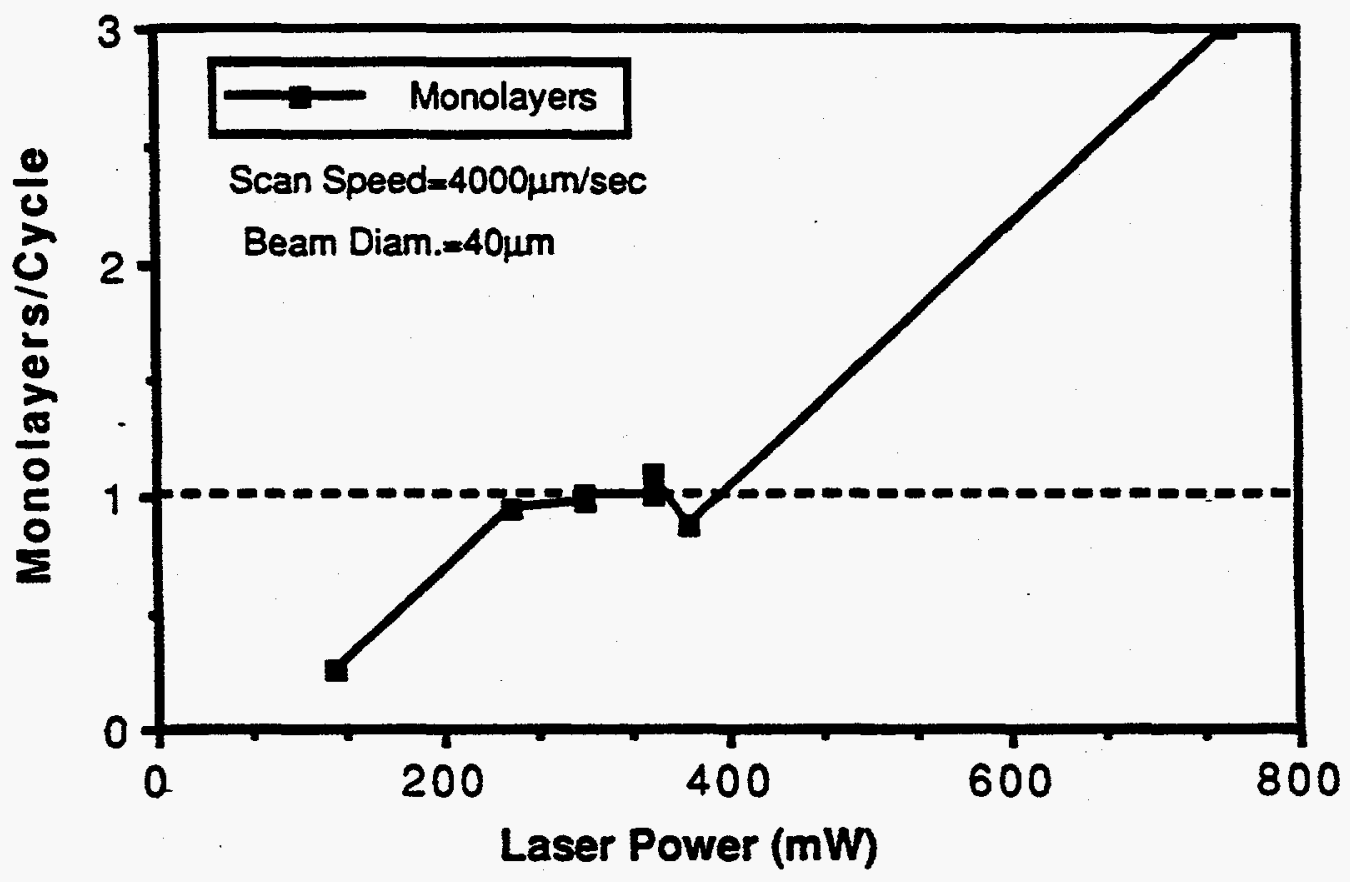

Figure 6.8

Growth rate as a function of laser power for LALE of GaAs on Alo.3Ga0.7As. 


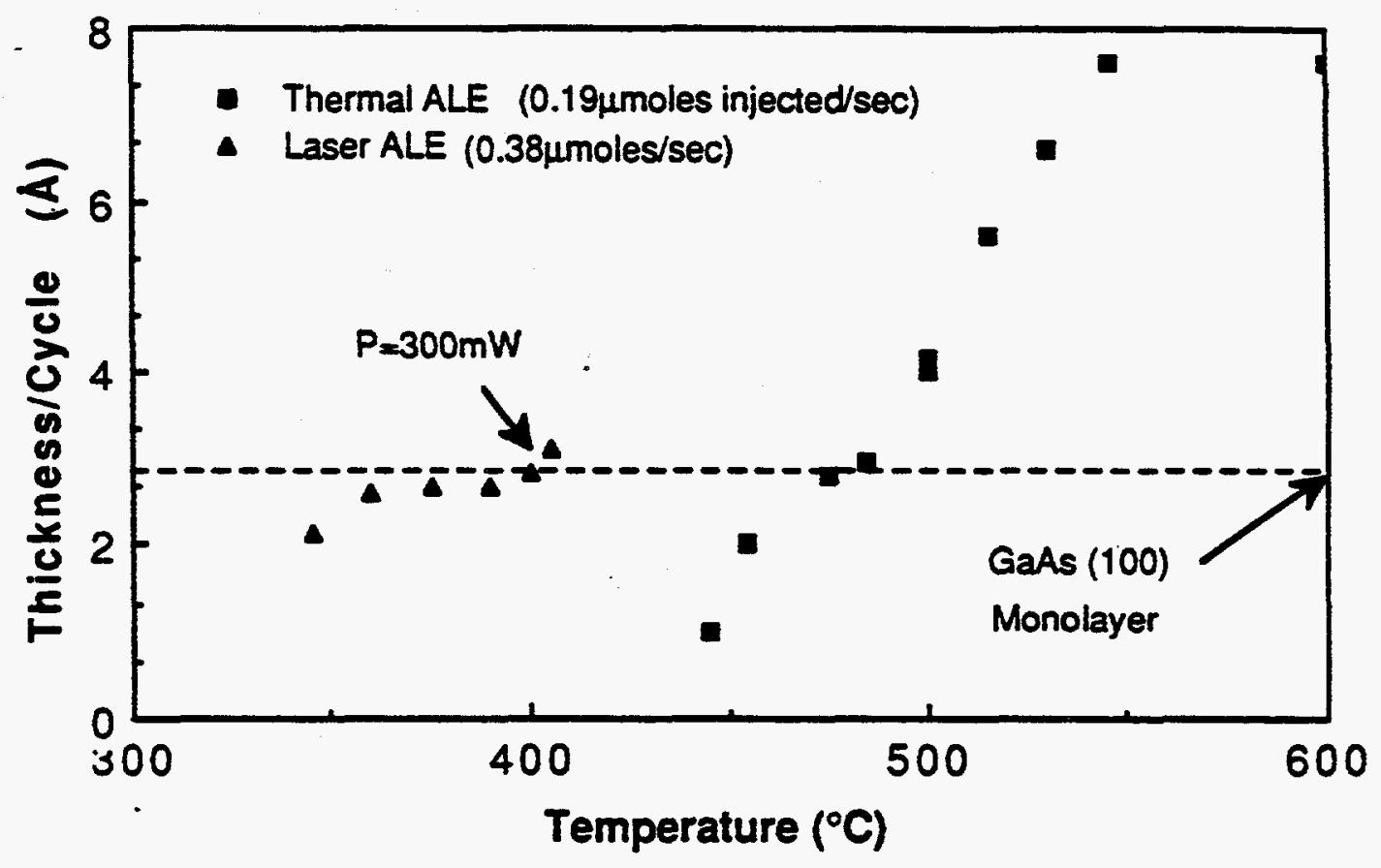

Figure 6.9.

Temperature dependence of Thermal and Laser ALE. Note independence of LALE growth rate over $50^{\circ} \mathrm{C}$ range which is indicative of photocatalytic mechanism. 


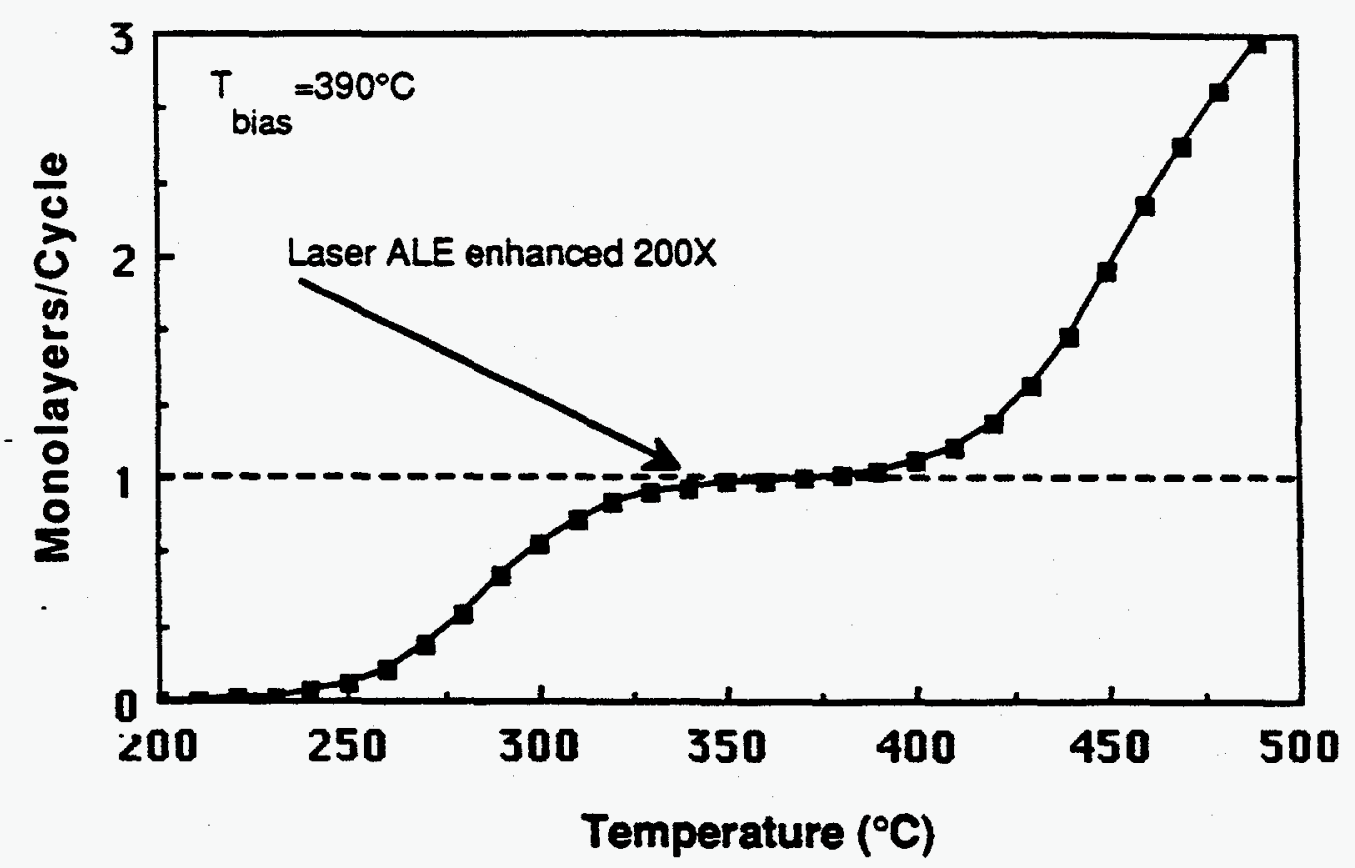

Figure 6.10

Laser ALE model predicts saturated growth over an range of temperatures as was observed in experimental results. The model uses a $300 \mathrm{~mW}$ laser beam with a $40 \mu \mathrm{m}$ diameter scanned at $4000 \mu \mathrm{m} / \mathrm{sec}$.

Further support for surface photoassisted process can be seen in a comparison of the scanned LALE process demonstrated in this work to the continuous LALE of Aoyagi et al. 8 The growth rate for the small spot sizes scales proportionally with power density in comparison to the larger spot sizes used by Aoyagi. Table 6.1 provides a comparison of the two techniques. In order to compare the techniques we use the "effective" growth rate which uses the effective exposure time " $\Delta \tau$ " of the laser on the spot area. Also evident is the observation that approximately the same total number of photons per surface area is necessary in the two cases. 
Rearranging equation 6.6 and integrating predicts equivalent deposited thicknesses for the different spot sizes and power densities:

$$
R_{\text {sur }}=\frac{d z}{d t}=\frac{\eta \theta\left(2 \sigma_{a} P_{L}\right)}{\left(h v \pi w_{0}^{2}\right)}
$$

Integrating:

$$
\int_{0}^{z(t=\tau)} d z=\int_{0}^{\Delta \tau} \frac{\eta \theta\left(2 \sigma_{a} P_{L}\right)}{\left(h v \pi w_{0}^{2}\right)} d t
$$

The deposited thickness is then equal to :

$$
z=\left(\frac{\eta \theta\left(2 \sigma_{a} P_{L}\right)}{\left(h v \pi w_{0}^{2}\right)}\right) \Delta \tau
$$

Since the "effective growth rate" of LALE scales proportionally with laser power density, as shown in Table 6.1, we can conclude that a surface photoassisted process is the dominant mechanism operative in LALE. This is evident when we compare the surface photoassisted rate equation 6.20 , to the photolytic gas phase equation 6.11 . The observation that growth is only occurring on the surface and no deposition is visible on the quartz windows is further support for a surface photoassisted process. We have also attempted to LALE deposit structures with the use of a Nd:YAG $(1.06 \mu \mathrm{m})$ laser. Below-bandgap irradiation yielded polycrystalline deposits with no sign of a saturated growth mechanism. Therefore, laser wavelengths larger than the band-gap of GaAs are 
deemed necessary presumably because they generate electron hole pairs necessary for a surface photocatalytic reaction.

\section{Table 6.1 Comparison of LALE Techniques}

\section{Deposited}

Thickness

(T)

"Effective Growth

Power

\#Photons Density $\mathrm{cm}^{2}$ Cycle

Spot

CW LALE rate" $(T / \Delta \tau)$

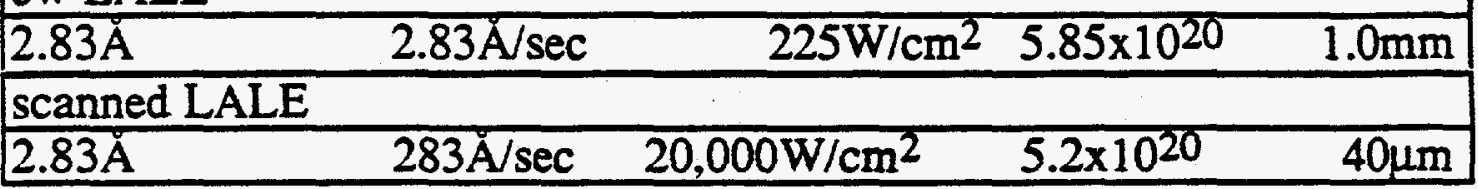

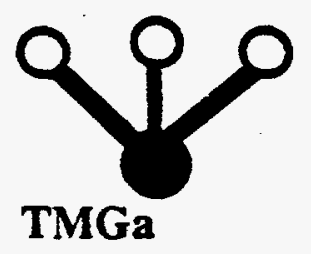

\section{$\mathbf{h} \vee>\mathbf{E}_{\mathbf{g}}$}

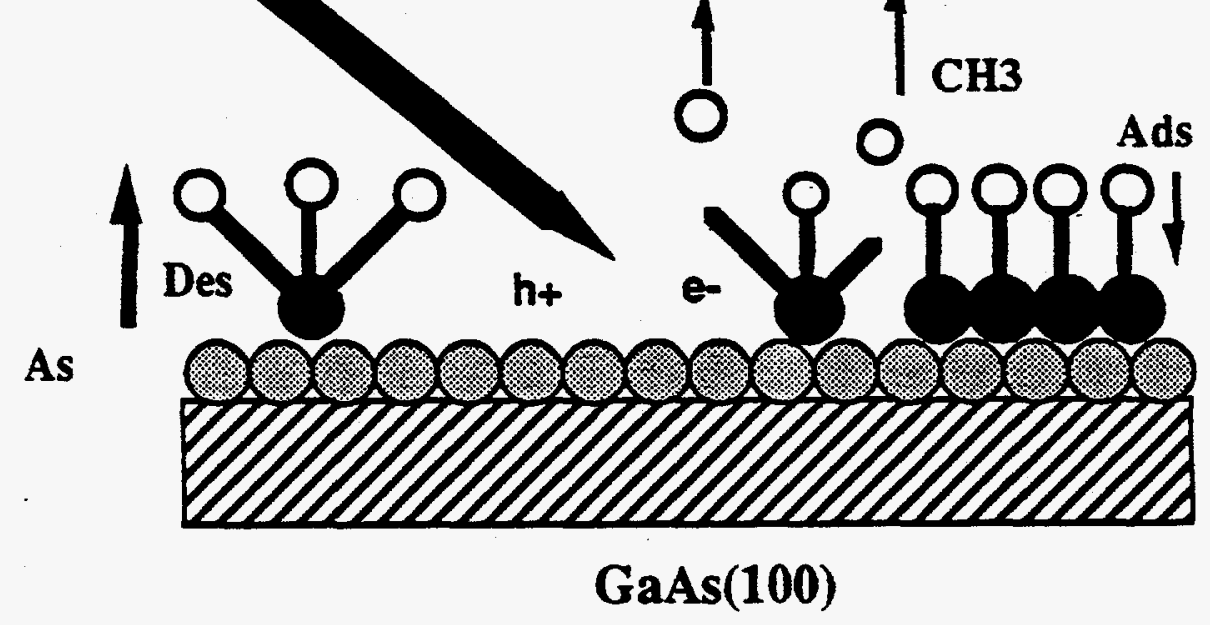

Figure 6.11

Photocatalytic Model for catalytic chemisorption of gallium methyl molecules on a $\mathrm{GaAs}$ surface. 


\subsubsection{Laser Heating Model}

A temperature rise of $40^{\circ}-100^{\circ} \mathrm{C}$ has been calculated for our conditions by using a model developed by Cline and Anthony. ${ }^{10}$ We use aGaussian beam profile to calculate the spatial temperature distribution near the incident spot as a function of substrate thermal properties, beam size and power, and scan speed. As shown in figure 6.11. a temperature rise of $70^{\circ} \mathrm{C}$ is predicted for a $50 \mu \mathrm{m}$ spot with $200 \mathrm{~mW}$ of incident power. This yields a power density of $10,000 \mathrm{~W} / \mathrm{cm}^{2}$. This temperature rise is greater then the $40^{\circ} \mathrm{C}$ calculated for the continuously irradiated LALE experiments. However, as we have shown in our model the fact that we scan our laser beam changes the kinetics of the surface reactions.

The equation for heat flow in a solid heated by a laser of power density $Q$ is given as:

$$
\frac{\partial T}{\partial t}-\frac{\partial}{\partial x}\left(\kappa \frac{\partial T}{\partial x}\right)-\frac{\partial}{\partial y}\left(\kappa \frac{\partial T}{\partial y}\right)-\frac{\partial}{\partial z}\left(\kappa \frac{\partial T}{\partial z}\right)=\frac{Q}{C_{p}}
$$

where $K$ is the thermal diffusivity, and $C_{p}$ the specific heat capacity per volume.

This model uses a moving gaussian intensity distribution normalized to give a total power of $P_{1}$ for a spot radius $R$ of the form: 


$$
Q=P_{L}\left(\frac{\exp \left(-\frac{\left(\left(x-v^{2}\right)+y^{2}\right)}{2 R^{2}}\right)}{2 \pi R^{2}}\right)\left(\frac{h(z)}{\lambda}\right)
$$

where $h$ is the absorption depth and $h(z)=1$ for $0<z<\lambda$, and $h(z)=0$ for $z>\lambda$.

Heat flow from the laser at $x^{\prime} y^{\prime} t^{\prime}$ on the surface influences the temperature at $\mathrm{x}, \mathrm{y}, \mathrm{z}$ in the material at a later time $\mathrm{t}$ is given as:

$$
T=\int_{-\infty}^{t} \int_{-\infty}^{\infty} \int_{-\infty}^{\infty} \int_{-\infty}^{\infty}\left(\frac{Q}{C_{p}}\right)\left(x^{\prime} y^{\prime} z^{\prime} t^{\prime}\right) G\left(x^{\prime} y^{\prime} z^{\prime} t^{\prime} \mid x y z t\right) d x^{\prime} d y^{\prime} d z^{\prime} d t^{\prime}
$$

Which $G$ is Green's function for the diffusion equation at the surface

$$
G=\frac{\exp \left(-\gamma^{2}\left\{4 D\left(t-t^{\prime}\right)\right\}^{-1}\right)}{4\left[\pi D\left(t-t^{\prime}\right)\right]^{3 / 2}}
$$

where $D^{-}$is the thermal diffusivity and:

$$
\gamma^{2}=(x-x)^{2}+\left(y-y^{\prime}\right)^{2}+(z-z)^{2}+(t-t)^{2}
$$

which yields at the surface where $\mathrm{z}=0$, and $\mathrm{t}=0$ the following integral.

$$
T=\frac{P_{1}}{C_{p}} \int_{0}^{\infty} \frac{\exp \left(-\left(\frac{\left((x+v)^{2}\right)}{\left(2 R^{2}+4 D t^{\prime}\right)}+\frac{z^{2}}{\left(4 D t^{\prime}\right)}\right)\right)}{\left(2 R^{2}+4 D t^{\prime}\right) \sqrt{\pi^{3} D t^{\prime}}} d t^{\prime}
$$


By transforming to dimensionless variables this integral can be further simplified to yield:

$$
T(x, y, z)=\frac{P_{1}}{C_{p} D R} \int_{0}^{\infty} \frac{\exp (-H)}{\sqrt{2 \pi^{3}}\left(1+\mu^{2}\right)} d \mu
$$

where the following substitution makes the expression readily evaluated by numerical analysis:

$$
H=\frac{\left(X+\frac{\rho}{2 \mu^{2}}\right)^{2}+Y^{2}}{2\left(1+\mu^{2}\right)}+\frac{Z^{2}}{2 \mu^{2}}
$$

The dimensionless variables are:

$$
\left.\mu^{2}=\left(2 D t^{\prime}\right) / R, \rho=R / D V\right), X=x / R, Y=y / R, Z=z / R
$$




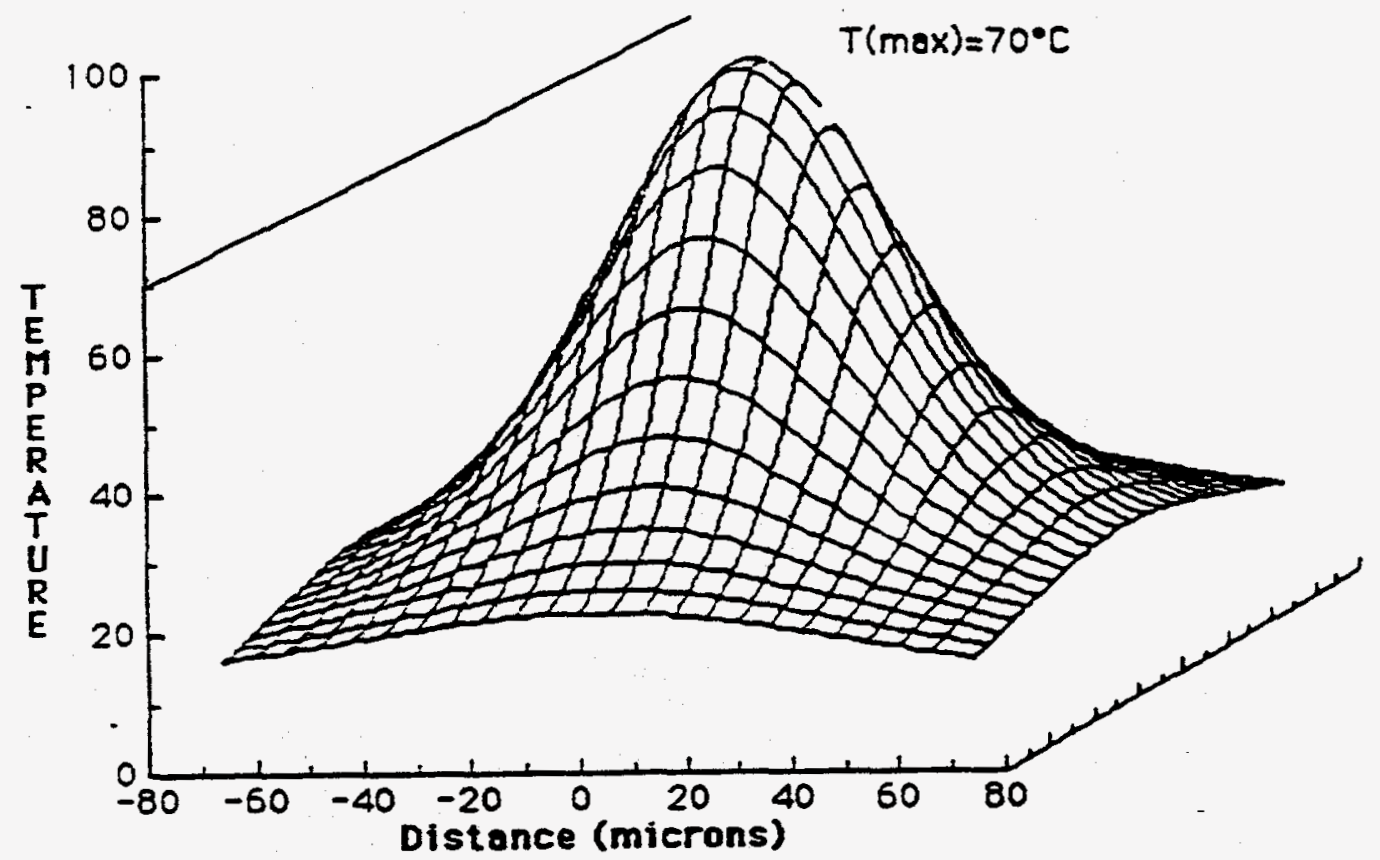

Figure 6.12

Calculated temperature rise for $50 \mu \mathrm{m}$ diameter laser beam at $200 \mathrm{~mW}$ scanned at $4000 \mu \mathrm{m} / \mathrm{sec}$. 


\subsubsection{Theoretical Limits on LALE Spatial Resolution}

Theoretically the spatial resolution of LALE deposits will be given by either the diffraction limited optical resolution or the thermal diffusion length. The diffraction limited optical spot size (FWHM) is given by:

$$
\delta_{0}=\frac{1.03 \lambda}{\text { N.A }}
$$

which yields a FWHM of $0.7 \mu \mathrm{m}$ for an immersion objective (N.A.=1.33). If the thermal diffusion length, $\mathrm{L}_{\mathrm{t}}=\left[2 \mathrm{Dt} \mathrm{t}_{\mathrm{p}}\right]^{0.5}$, limits the spatial resolution we can minimize the deposits size by decreasing the laser exposure time. For GaAs the thermal diffusivity is $0.072 \mathrm{~cm}^{2} / \mathrm{sec}$ at $390^{\circ} \mathrm{C}$ which yields diffusion lengths of less than $1.2 \mu \mathrm{m}$ when scan speeds are increased to yield an effective 100 nanosecond exposure. It is highly unlikely that photogenerated carrier diffusion lengths will limit the resolution, since the surface recombination velocities at the GaAs surface are very large $\left(10^{6} \mathrm{~cm} / \mathrm{sec}\right) .11$

Surface migration of the adsorbed species is another factor which might limit the deposit resolution. However, surface migration of the photogenerated adsorbate should be minimal because of the strong bonds formed in chemisorption and low temperatures involved. Recently, coworkers have observed that surface migration lengths in conventional 
MOCVD appear to be less than $5 \mu \mathrm{m}$ at $750^{\circ} \mathrm{C} .12$ They also observe a decrease in the mobility of the surface species upon lower the temperature to $650^{\circ} \mathrm{C}$. Surface diffusion should decrease exponentionally with temperature. Therefore, it is highly probably that surface migration at $400^{\circ} \mathrm{C}$ will be minimal and $1 \mu \mathrm{m}$ deposits readily achievable.

\subsection{CONCLUSIONS}

The "fast writing" potential of LALE to selective deposit GaAs on $\mathrm{Al}_{0.3 \mathrm{Ga0}} \mathrm{AAs}$ is demonstrated. The resultant process is carried out in a four chamber atmospheric MOCVD reactor which can also grow conventional MOCVD layers. High quality materials and heterostructures have been accomplished by a hybridization of thermal ALE and MOCVD. The demonstration in the previous chapter on fabrication of high quality quantum wells and quantum well lasers with low threshold current densities, indicates that this process should eventually result in the selective deposition of opto-electronic and electronic components on the chip in a single run. 
REFERENCES-CHAPTER 6

1 T. Cacouris, G. Scelsi, R. Scarmozzino, R. M. Osgood, Jr., R. R. Krchnavek, Laser and Particle Beam Chemical Processing for Microelectronics, edited by D. Ehrlich, G. S. Higashi, and M. M. Oprysko (MRS Research Symposium Proceedings, Pittsburgh 1987) Vol 101, p.43.

2 A.Doi , Y. Aoyagi, S. Namba, Appl. Phys. Lett. 49, 785 (1986).

3 S. P. DenBaars, P. D. Dapkus, J. S. Osinski, M. Zandian, C. A. Beyler, and K. M. Dzurko, D6, 15th International Symposium on Gallium Arsenide and Related Compounds, Atlanta, GA (1988).

4 S. R. Morrison, The Chemical Physics of Surfaces, (Plenum Press, New York 1977), p.301.

5 V. F. Kiselev, O. V. Krylov, Adsoprtion Proccesses on Semiconductors and Dielectric Surfaces (Springer-Verlag, Berlin 1985) p.785.

$6 \quad$ M. Lax, J. Appl. Phys. 48, 3919 (1977).

7 C. J. Chen, J. Vac. Sci. Technol., A5(6), 3386 (1987).

8 J. Nishizawa, T. Kurabayashi, H. Abe and A. Nozoe, Surface Science, 185, 249 (1987).

9 Y. Aoyagi, A. Doi, S. Namba, J. Vac. Sci. Technol., B5, 1460 (1987).

10 H. E. Cline, and T. R. Anthony, J. Appl. Phys., 48, 3897 (1977).

11 H. J. Hovel, Semiconductors and Semimetals. Vol 11 Solar Cells, (Academic Press, New York 1975) p.15. 
12 K.M. Dzurko, E. P. Menu, P. D. Dapkus, D-11, 15th International Symposium on Gallium Arsenide and Related Compounds, Atlanta, GA (1988). 


\section{CHAPTER 7 \\ CONCLUSIONS AND RECOMMENDATIONS FOR FUTURE RESEARCH}

\subsection{Conclusions from this Work}

In this work, atomic layer epitaxy of GaAs, AlAs and AlGaAs has been demonstrated. Saturated monolayer growth of GaAs and AlAs has been observed by using metalorganic precursors at growth temperatures where kinetics of surface reactions dominate the deposition process. These surface reactions can be driven by thermal processes alone or by photo-assisted ALE. We have established some of the inherent advantages of the ALE technique by demonstrating the "digital" nature of the deposition process and growing extremely uniform ultrathin layers. We have measured ALE QW thickness variations of less than one monolayer across an entire sample grown in our small residence time reactor.

We have established for the first time that ALE can be hybridized with conventional MOCVD to grow device quality structures. This has been demonstrated by the operation for the first time of an injection laser with an active grown by ALE. 1 Quantum well lasers with threshold current densities as low as $380 \mathrm{~A} / \mathrm{cm}^{2}$ have been achieved. Low temperature PL of single quantum wells (SQWs) grown by a hybridization of ALE and conventional MOCVD exhibit narrow linewidth intrinsic luminescence. These results suggest that ALE will play 
an important role in the fabrication of heterojunction devices with critical layer thickness requirements.

In laser assisted ALE, selective area growth can occur by photolytically controlled surface reactions at substrate temperatures well below that at which purely thermal ALE will occur. The "fast writing" potential of LALE to selective deposit GaAs at scan speeds of $4000 \mu \mathrm{m} / \mathrm{sec}$ is demonstrated. 2 We have employed a scanned laser beam with spot sizes

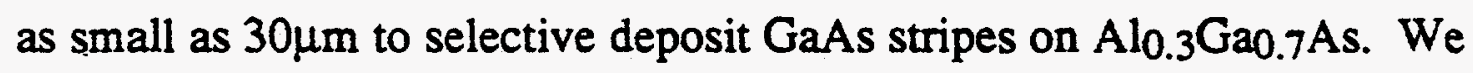
observe perfect monolayer saturation in the growth rate over an order of magnitude range of TMGa fluxes. Saturated monolayer LALE growth also occurs over a variety of power densities and a $50^{\circ} \mathrm{C}$ range of growth temperatures. The LALE deposits exhibit smooth mirror like morphology with flat-tops thickness profiles indicative of a mono-atomic growth mechanism.

The ALE and LALE of this work was accomplished using conventional MOCVD precursors and vent/run MOCVD reactors modified to allow alternate exposures of the substrate to the column III and column $\mathrm{V}$ precursors. In one case the reactant exposures are separated temporally by altemately injecting the reactants for one second each into a short residence time horizontal reactor. In the second design reactant exposures are separated spatially by utilizing a four chamber vertical reactor in which the substrate is rotated between continuous reactant flows. Under appropriate conditions one monolayer of the III-V 
compound is formed uniformly in thermally driven ALE and only in the region of laser excitation for LALE. Both reactors are designed with the ability to grow conventional MOCVD layers thus allowing a hybridization of ALE and MOCVD, in the same reactor.

The kinetics for the ALE process have been investigated and are shown to behave in accordance with a first order adsorption model. The kinetics of the process are controlled by the chemisorption of the appropriate precursor on the GaAs surface. Chemisorption of a gallium methyl adsorbate appears to be the controlling step in the growth process. The formation of the adsorbed species, most likely $\mathrm{GaCH}_{3}$, can be achieved by purely thermal processes or photo-assisted process. In thermal ALE removal of the first methyl radical in the gas or surface phase is theorized to be the rate limiting step. In laser assisted ALE selective area growth occurs by photochemical surface reactions of either photocatalytic or pyrolytic nature. The addition of the As monolayer appears to occur by the heterogeneous reaction of arsine with the $\mathrm{Ga}$ adsorbate. 
By using reaction rates measured by sampled gas infrared spectroscopy we obtain good qualitative agreement between experimental results and predicted behavior from the first order ALE model. The pyrolysis of TMGa was found to occur in stages, with the first two methyl groups releasing in the $350-450^{\circ} \mathrm{C}$ range. It is not definite that all stages of alkyl elimination are homogeneous. The only gas phase by-product of the decomposition observed is $\mathrm{CH}_{4}$. Arsine decomposition on the other hand is strongly catalyzed by wall deposits, GaAs surfaces, and gaseous mixtures with TMGa. The activation energy for $\mathrm{AsH}_{3}$ decomposition is reduced for the reactor at the GaAs surface. We emphasize that these catalytic reactions play an important role in the MOCVD and ALE growth of $\mathrm{GaAs}$ and must be taken into account when modeling a large scale reactor.

Alternative gaseous arsenic sources that decompose at low temperatures were also studied. Thermal decomposition of TEAs in $\mathrm{H}_{2}$ occurs via a $B$-hydride elimination mechanism, producing DEAsH and $\mathrm{C}_{2} \mathrm{H}_{4}$ as the organic by-products. Thermal decomposition of DEAsH was observed to produce predominantly $\mathrm{C}_{2} \mathrm{H}_{6}, \mathrm{C}_{2} \mathrm{H}_{4}$ and a small quantity of arsine. Both DEAsH and TEAs decomposition was discovered to be catalyzed by the addition of single crystal $\mathrm{GaAs}(100)$ surfaces to the reactor. Since DEAsH does not form an adduct with TMGa, whereas TEAs does, DEAsH is concluded to be the more attractive group V alternative source. Application of these sources to ALE should be studied, since they are significantly less toxic than arsine and decompose at lower temperatures 


\subsection{Recommendations for Future Research}

Selective area epitaxy by laser assisted ALE has great potential for the integration of optoelectronic and electronic devices on the same chip. Optimization of the LALE process for the growth of GaAs/AlGaAs and InP/InGaAs heterostructures would allow the in situ deposition of heterostructure devices with differing epitaxial structures requirements. Growth and fabrication of buried heterostructure lasers by selectively depositing the GaAs active region using LALE is an attractive process for the demonstration of low threshold lasers. In addition, integration of multiple wavelength lasers on the same chip will be possible. The minimum spatial resolution achievable by LALE should be studied. Theoretically spatial dimensions of $1-2 \mu \mathrm{m}$ are obtainable if optimum scan rates and optical components are used. Surface migration of the photogenerated adsorbate should be minimal because of the strong bonds formed in chemisorption and low temperatures involved.

The manufacturing benefits of the in situ aspects of the LALE process for multiple wafer throughput is particularly attractive. In this study we have established the "fast writing" potential of LALE for depositing small dimension structures. An extension of this work would be the demonstration of multiple stripe lasers fabricated by a parallel optical laser writing setup. Another attractive process would be the the use of holographic projection for the concurrent deposition of multiple devices. Fabrication of heterojunction bipolar transistors (HBTs) 
requires deposition of base, emitter, and collector regions. We envision a LALE process using a set of three holographic plates to deposit the different epitaxial layers. Figure 7.1 illustrates the in situ deposition of multiple transistor elements on the same wafer.

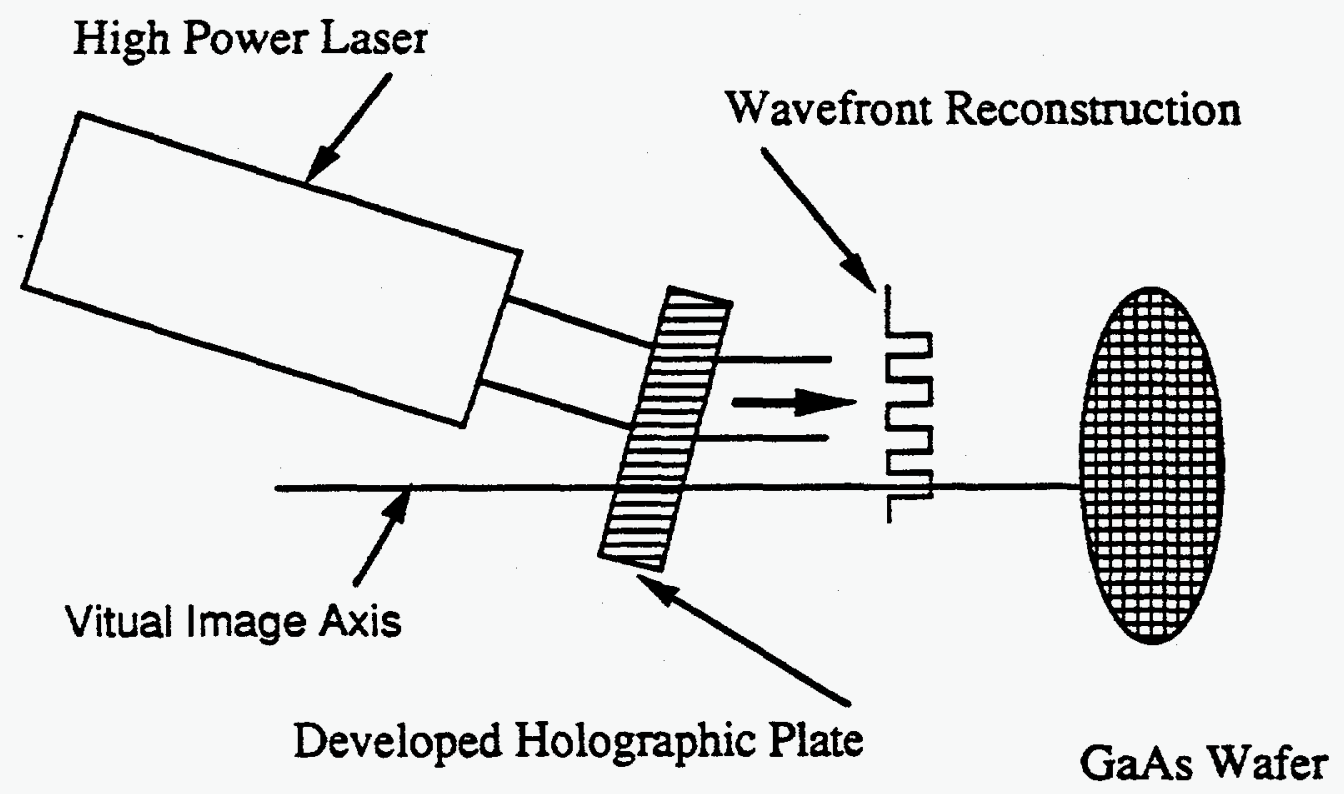

Figure 7.1

LALE process using holographic projection for multiple device fabrication. 
The uniformity benefits inherent in the ALE process should be applied to large area epitaxial growth. By utilizing lower pressure short residence time reactors large area deposition of several III-V semiconductors should be possible by pulsed gas injection. Rotation of wafers in a small cross-section four chamber reactor could also yield highly uniform large area deposits. One can envision high reactant utilization if the wafers are rotated in relatively stagnant flows of column III and column V, under conditions of surface catalyzed reactions only. By using cylindrical lenses to induce monolayer photodeposition on the GaAs wafer surfaces $100 \%$ utilization of sources is achievable. Such a process could be obtained by simply increasing the power densities and rotation rates in demonstrated in our work by a factor of 10 . Figure 7.2 shows a proposed design to achieve such an ambitious process. 


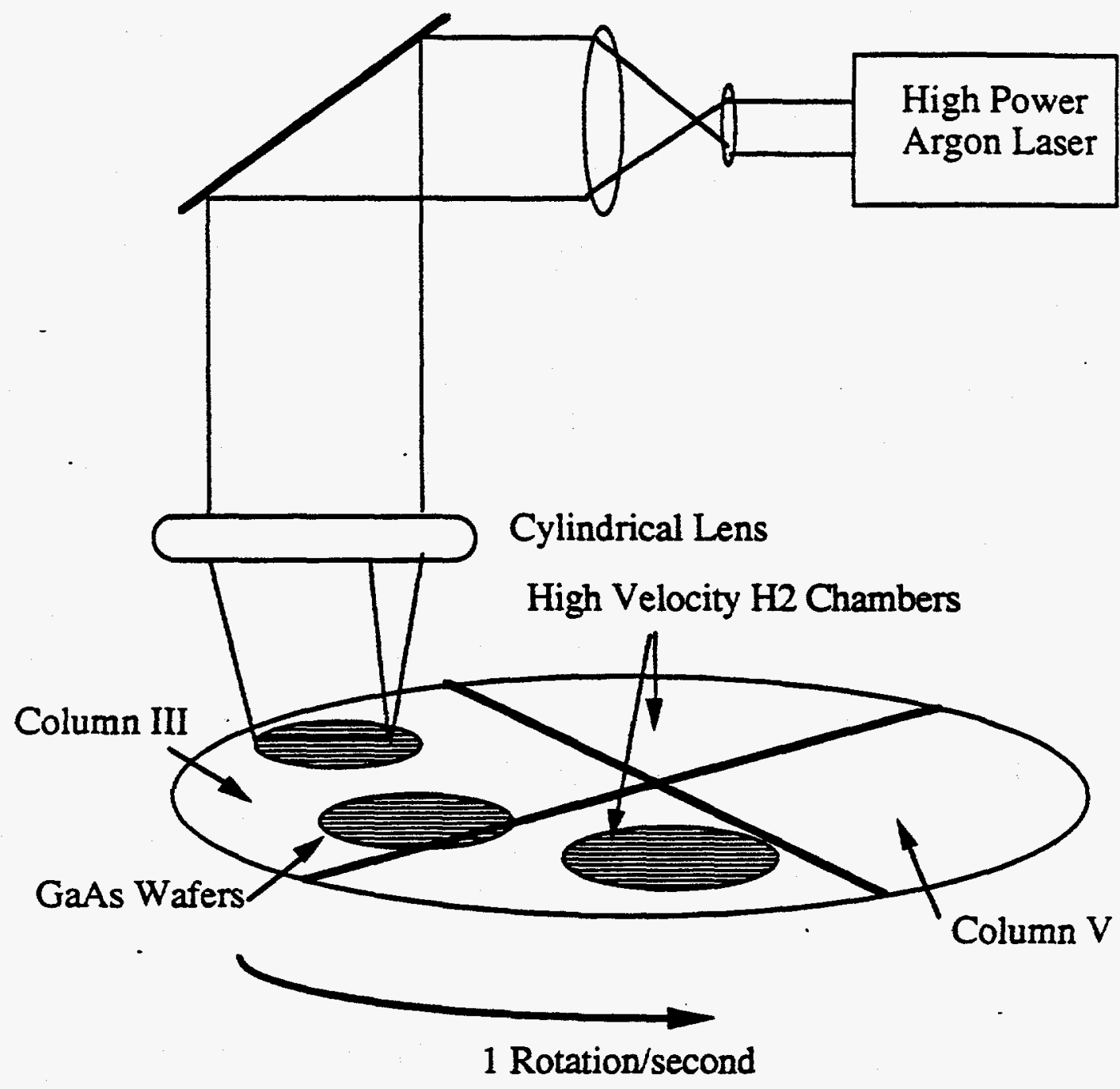

Figure 7.2

Multiple Wafer LALE deposition process 
Another aspect of ALE that warrants research is yrowth on structured substrates. Coworkers have recently demons ated low threshold lasers with small active regions grown on structure substrates by conventional MOCVD in our laboratory. 3 By using LA .E to grow the selective area GaAs/AlGaAs and the etched features to def e the small active region, localized low threshold lasers could be deposite. Coupling this technique with LALE of transistors structures wou 1 result in integrated electronic and opto-electronic devices on same chif

Many other devices and materials would greatly ben: it from the advantages of ALE. The ability to grow extremely unifc $m$ ultrathin layers with the digital ALE method should have inpact on si 'eral device structures. Successful fabrication of high electron mobilit transistors (HEMTs) requires the ability to grow highly uniform monc ayer abrupt interfaces. ${ }^{4}$ Quantum wells, as well as devices incorpc ating these structures, require the ability to produce thin layers ith abrupt interfaces. In the quantum well injection laser the thres old current density and lasing wavelength are strongly dependent upon thi active layer thickness-in the $10-60 \AA$ range.5 ALE growth of GaAs on ii and Ge is another area of future research. It is highly probable that ALI will reduce the dislocation density and antiphase domains which appear hen a III-V zincblende material is deposited on the diamond lattice of Si.o: Ge. 
n conclusion, this work has demonstrated several advantages of the $f, E$ and LALE techniques. Monolayer thickness uniformity in quant $\mathrm{n}$ well structures has been accomplished. We have achieved the first I -V device fabricated by ALE. Quantum well injection laser with activ $\epsilon$-egions grown by ALE operate with threshold current densities as low a $380 \mathrm{~A} / \mathrm{cm}^{2}$. Low temperature PL of single quantum well (SQWs) with stive regions grown by ALE exhibit narrow linewidth intrinsic lumir scence. LALE shows great potential as a fabrication technology for in :grated optoelectronics.

\section{REF: RENCES-CHAPTER 7}

1 3. P. DenBaars, C. A. Beyler, A. Hariz, and P. D. Dapkus, Appl. 'hys. Lett., 51, 1530 (1987).

2 3. P. DenBaars, P. D. Dapkus, J. S. Osinski, M. Zandian, C. A. 3eyler, and K. M. Dzurko, D6, 15 th International Symposium on Iallium Arsenide and Related Compounds, Atlanta, GA (1988).

$3 \quad$ J.M. Dzurko, E. P. Menu, P. D. Dapkus, D-11, 15th International jymposium on Gallium Arsenide and Related Compounds, Atlanta, JA (1988).

4 ว. D. Dapkus, J. Crystal Growth, 68, 345, (1984).

5 -. J. Mawst, M. E. Givens, C. A. Zmudzinski, M. A. Emanuel, and J. J. Coleman, IEEE J. Quantum Electron., QE-23, 696, 1987). 


\section{BIBLIOGRAPHY}

V. Aebi, C. B. Cooper, R. L. Moon, and R. R. Saxena, J. Crystal Growth 55, 517 (1981).

Z. I. Alferov, V. M. Andreev, D. Z. Garbuzov, Y. V. Zhilyaev, E. P. Morozov, E. L. Portinoi, and V. G. Trofim, Sov. Phys. Semicond. 4, 1573 (1971) [Translated from Fiz. Tekh. Poluprovodn. 4, 1826 (1970)].

Y. Aoyagi, A. Doi, S. Iwai, and S. Namba, J. Vac. Sci. Technol. B5(5), 1460 (1987).

R. Z. Bachrach, Crystal Growth, 2nd edition(London, Pergamon Press Ltd., 1980) B. R. Pamplin ed., pp. 221-271.

S. J. Bass, J. Crystal Growth 31, 172 (1975).

S. J. Bass, J. Crystal Growth 44, 29 (1978).

G. Bastard, Phys. Rev. B, 24, 5693 (1981).

S. W. Benson, Thermochemical Kinetics, 2nd Ed, (John Wiley \& Sons, New York, 1976) pp.17-85.

S. Berkman, V. S. Ban, and N. Goldsmith, Heteroiunction Semiconductors for Electronic Devices, eds., G. W. Cullen and C. C. Wang (Śpringer-Verlag, Berlin 1979) p. 264.

D. C. Bertolet, J. K. Hsu and K. M. Lau, J. Appl. Phys., 62,120 (1987).

R. Bhat, J. Electron. Mater. 14, 433 (1985).

R. Bhat, J. R. Hayes, E. Colas, and R. Esagui, IEEE Electron Device Lett., 9, 442 (1988).

R. Bhat, M.A. Koza, and B.J. Skromme, Appl. Phys. Lett. 50, 1194 (1987). 
T. Cacouris, G. Scelsi, R. Scarmozzino, R. M. Osgood, Jr., R. R. Krchnavek, Laser and Particle Beam Chamical Processing for Microelectronics, edited by D. Ehrlich, G. S. Higashi, and M. M. Oprysko (MRS Research Symposium Proceedings, Pittsburgh 1987) Vol 101, p.43.

H. C. Casey, Jr and M. G. Panish, Heterostructure Lasers, (New York, Academic Press, 1978) pp.167-176.

C. J. Chen, J. Vac. Sci. Technolo., A5(6), 3386 (1987).

H. E. Cline, and T. R. Anthony, J. Appl. Phys., 48, 3897 (1977).

E. Colas, R. Bhat, B. Skromme, Paper D-8, International Symposium on GaAs and Related Compounds, Atlanta, GA 1988.

J. J. Coleman, and P. D. Dapkus, Gallium Arsenide

Technology,(Indianapolis, Howard W. Sams \& Co., Inc., 1985) D. K. Ferry ed., p.91.

A. D. Danner, PhD. Dissertation, University of Southern California (1987) p.72-74.

P. D. Dapkus, Class Notes, EE-599, Heterostructure Materials and Devices, University of Southern Califomia, (1986).

P. D. Dapkus, J. Crystal Growth 68, 345 (1984).

S. P. DenBaars, B. Y. Maa, P. D. Dapkus, A. D. Danner and H. C. Lee,J. Crystal Growth, 77, 188(1986).

S. P. DenBaars, H. C. Lee, A. Hariz, P. D. Dapkus, N-3, Electronic Materials Conference, Santa Barbara, (1987).

S. P. DenBaars, C. A. Beyler, A. Hariz, and P. D. Dapkus, Appl. Phys. Lett., 51, 1530 (1987).

S. P. DenBaars, P. D. Dapkus, C. A. Beyler, A. Hariz, and K. M. Dzurko, J. Crystal Growth, 202T, Proceedings of the IV International Conference on OMVPE, Hakanoe, Japan (1988). 
S. P. DenBaars, P. D. Dapkus, J. S. Osinski, M. Zandian, C. A. Beyler, and K. M. Dzurko, D6, 15th International Symposium on Gallium Arsenide and Related Compounds, Atlanta, GA (1988).

R. Dingle, Festkorperprobleme XV, 21 (1975).

A. Doi, Y. Aoyagi, S. Namba, Appl Phys. Lett. 48, 1787, (1986).

A.Doi , Y. Aoyagi, S. Namba, Appl. Phys. Lett. 49, 785 (1986).

R. D. Dupuis, P. D. Dapkus, R. D. Yingling, and L. A. Moudy, Appl. Phys. Lett., 31, 201 (1977).

R. D. Dupuis and P. D. Dapkus, Appl. Phys. Lett., 32, 472 (1978).

T. Fukunaga, K. L. I. Kobayashi, and H. Nakashima, Surface Sci., 174, 71 (1986).

G.H. Geiger, D. R. Poirier, Transport Phenomena in Metallurgy, (Addison-Wesley Publishing Co., Menlo Park, CA 1980) pp.8-63.

B. K. Gilbert, Gallium Arsenide Technology, (Indianapolis, Howard W. Sams \& Co., Inc., 1985) D. K. Ferry ed., p.28.

C. H. L. Goodman and M. V. Pessa, J. Appl. Phys. R65, 60 (1986).

S.R. Gunn, Inorg. Chem., 11, 796 (1972).

M. A. Herman, M. Vulli, and M. Pessa, J. Cryst. Growth 67,339 (1985).

N. Y. Holonyak, R. M. Kolbas, R. D. Dupuis, P. D. Dapkus, IEEE J. Quantum Electronics, QE-16, 170 (1980).

S. Ito, T. Shinohara, and Y. Seki, J. Electrochem. Soc., 120, 1419 (1972).

M. G. Jacko and S. J. W. Price, Can. J. Chem. 41, 1560(1963).

S. J. Jeng, C. M. Wayman, G. Costrini, and J. J. Coleman, Materials Lettters 2, 359 (1984). 
H. Kakibayashi and F. Nagata, Surface Sci, 74, 84 (1986).

D. Kasemet, C. Hong, N. B. Patel, and P. D. Dapkus, IEEE J. of Quantum Elec., QE-19,1025 (1983).

H. Kawai, I. Hase, K. Kaneko and N. Watanabe, J. Crystal Growth 68,508 (1984).

T. F. Keuch, and E. Veuhoff, J. Crystal Growth, 68, 148 (1984).

V. F. Kiselev, O. V. Krylov, Adsorption Proccesses on Semiconductors and Dielectric Surfaces (Springer-Verlag, Berlin 1985) p.785.

N. Kobayashi, T. Makimoto, and Y. Horikoshi, Jap. J. Appl. Phys. 24, L962 (1985).

N. Kobayashi, T. Makimoto, and Y. Horikoshi, Appl. Phys. Lett, 50, 1435 (1987).

M. Kumagawa, H. Sunami, T. Terasaki, and J. Nishizawa, Japan J. Appl. Phys. 7, 1332 (1968).

D. M. Larsen, Phys. Rev. B8, 535 (1973).

M. Lax, J. Appl. Phys. 48, 3919 (1977).

H. C. Lee, PhD. Dissertation, University of Southern California, (1987) pp.106-130.

P. Lee, D. McKenna, D. Kapur and K.F. Jensen, J. Crystal Growth. 77,120 (1986).

I. R. Levine, Physical Chemistry, McGraw-Hill Publ. Co., (New York 1978) p.771.

M.R. Leys and H. Veenvliet, J. Crystal Growth, 55, 145 (1981).

T. Makimoto, Y. Yamauchi, and Y. Horikoshi, Paper D-7, International Symposium on GaAs and Related Compounds, Atlanta, GA 1988 
H. M. Manasevit, Appl. Phys. Lett. 12, 156 (1968).

H. M. Manasevit, J. Electrochem. Soc. 118, 647 (1971).

H. M. Manasevit and W. I. Simpson, J. Electrochem. Soc. 116, 1725 (1969).

H. M. Manasevit and K. L. Hess, J. Electrochem. Soc. 126, 2031 (1979).

L. J. Mawst. M. E. Givens. C. A. Zmudzinski, M. A. Emanuel, and J. J. Coleman, IEEE J. Quantum Electron., QE-23, 696, (1987).

S. R. Morrison. The Chemical Physics of Surfaces, (Plenum Press. New York 1977) p.301.

J. Nishizawa. H. Abe and T. Kurabayashi, J. Electrochem. Soc. 132. 1197 (1985).

J. Nishizawa. T. Kurabayashi,H. Abe, and N. Sakurai, J. Electrochem. Soc. 134, 945 (1986).

J. Nishizawa and T. Kurabayashi, J. Electrochem. Soc. 130, 413 (1983)

J. Nishizawa. T. Kurabayashi. H. Abe and A. Nozoe. Surface Science, 185, 249 (1987).

S. B. Ogale, A. Madhukar, F. Voillot, M. Thomsen, W. C. Tang, T. C. Lee, Y. Kim and P. Chen, Phys. Rev. B, 36.1662 (1987).

H. Okamato. Jap. J. Appl. Phys., 26, 315 (1987).

M. Pessa, P. Huttunen. M. A. Herman. J. Appl. Phys. 54, 6047 (1983).

M. Pessa. R. Makela, R. Suntola. Appl. Phys. Lett. 38, 131 (1980).

W. H. Petzke, V. Gottschalch and E. Butter, Krist. Tech. 9. 763 (1974).

D. H. Reep and S. K. Ghandi, J. Electrochem. Soc. 130. 675 (1983). 
W. Richter, "Physics of Metal Organic Chemical Vapour Deposition," Festkorperprobleme XXVI (Pergamon Piess, New York, 1986).

C. F. Schaus, J. R. Shealy, L. F. Eastman, B. C. Cooman and C. B. Carter, J. Appl. Phys. 59, 678 (1986).

Y. Seki, K. Tanno, K. Lida, and E. Ichiki, J. Electrochem. Soc. 122, 1108 (1975).

J. Singh K. K. Bajaj and S. Caudhuri, Appl. Phys. Lett. 44, 805(1984)

D.M. Speckman and J.P. Wendt, Appl. Phys. Lett. 50, 676 (1987).

K. Tamura, J. Phys. Chem. 59, 777 (1955).

V. P. Tanninen, M. Oikkoinen and T. Tuomi, Thin Solid Films 109, 283 (1983).

M. A. Tischler, N. G. Anderson, and S. M. Bedair, Appl. Phys. Lett. 49, 1199 (1986).

M. A. Tischler, N. G. Anderson, T. Katsuyama, and S. M. Bedair, Electronic Materials Conference, Boulder, CO, June (1985).

W. T. Tsang, Appl. Phys. Lett. 40, 217 (1982).

W.T. Tsang, Appl. Phys. Lett. 45,1234 (1984).

A. Usi and H. Sunakawa, Inst. Phys. Conf. Ser. No. 79, 753 (1985).

J. S. Yuan, C. C. Hsu, R. M. Cohen, and G. B. Stringfellow, Appl. Phys Lett., (1985).

M. Yoshida, H. Watanabe, and F. Uesugi, J. Electrochem. Soc. 130, 413 (1985).

M. Yoshida, H. Watanabe and F. Uesugi, J. Electrochem. Soc. 137, 677 (1985). 


\section{APPENDIX}

Appendix A

Integration of first order adsorption model

Integrating:

$$
\frac{d \theta}{d t}=k_{\text {ads }}(1-\theta)-k_{d e s}(\theta)+k_{G a}
$$

$$
\int_{0}^{\theta} \frac{d \theta}{\left(k_{\text {ads }}(1-\theta)-k_{\text {des }}(\theta)+k_{G a}\right)}=\int_{0}^{t} d t
$$

Rearranging:

$$
\int_{0}^{\theta} \frac{d \theta}{\left(\left[-\left(k_{\text {ads }}+k_{\text {des }}\right)\right] \theta+k_{\text {ads }}+k_{G a}\right)}=\int_{0}^{t} d t
$$

From Integral Tables:

$$
\int \frac{d x}{A x+B}=\frac{1}{A} \ln [A x+B]+C
$$

Substituting:

$$
\begin{gathered}
\text { Let }: A=\left[-\left(k_{\mathrm{ads}}+\mathrm{k}_{\text {des }}\right)\right] \\
B=\left(\mathrm{k}_{\mathrm{ads}}+\mathrm{k}_{\mathrm{Ga}}\right) \\
\left.\frac{1}{A} \ln [\mathrm{A} \theta+B]\right|_{0} ^{\theta(t)}=\mid \mathrm{t}_{0}^{t} \\
\ln [B]-\ln [A \theta+B]=-A t \\
\ln \frac{[B]}{[A \theta+B]}=-A t \\
\frac{[A \theta+B]}{[B]}=\exp (A t)
\end{gathered}
$$




$$
\begin{aligned}
& A \theta=[B] \exp (A t)-B \\
& \theta=\frac{B}{A}(\exp (A t)-1)
\end{aligned}
$$

Substituting values back in yields:

$$
\theta=-\left(\frac{k_{\mathrm{Ga}}+\mathrm{k}_{\mathrm{ads}}}{\mathrm{k}_{\mathrm{ads}}+\mathrm{k}_{\text {des }}}\right)\left[\exp \left[-\left(\mathrm{k}_{\mathrm{ads}}+\mathrm{k}_{\mathrm{des}}\right) \mathrm{t}\right]-1\right]
$$

Rearranging into final form:

$$
\theta=\left(\frac{k_{G a}+k_{\text {ads }}}{k_{\text {ads }}+k_{\text {des }}}\right)\left[1-\exp \left[-\left(k_{\text {ads }}+k_{\text {des }}\right) t\right]\right]
$$

\section{Appendix B}

\section{Kinematic Viscosity of Hydrogen}

The viscosity of nonpolar gases such as hydrogen gas can be calculated at any temperature by using Newton's law of viscosity corrected for the energy of interaction between molecules in the gas. The Lenard-Jones parameters are used to account for the potential energy of interaction between a pair of molecules in the gas. These parameters are the characteristic diameter of the molecule $\sigma\left(\mathrm{H}_{2}=2.915 \AA\right)$, and the collision integral $\Phi_{\eta}\left(\mathrm{H}_{2}=0.7432\right) .1$ Using these parameters the viscosity of nonpolar gases can be determined from:

$$
\eta=2.67 \times 10^{-5} \frac{\sqrt{\mathrm{MT}}}{\sigma^{2} \Phi_{\eta}}
$$


where $M$ is the gram molecular weight, $\eta$ is the viscosity in poises, and $T$ in ${ }^{\circ} \mathrm{K}$. For hydrogen at $455^{\circ} \mathrm{C}$, this yields a viscosity of $1.28 \times 10^{4}$ poise ( poise $=\mathrm{gm} / \mathrm{cm}-\mathrm{sec}$ ). For the boundary layer calculations we need to use the kinematic viscosity which is a measure of the momentum diffusivity:

$$
v^{\prime} \equiv \frac{\eta}{\rho}
$$

where $\rho$ is the density of the gas $\left(\rho=8.49 \times 10^{-5} \mathrm{gm} / \mathrm{cm}^{3}\right.$ for hydrogen). The boundary layer thickness is determined from equation 4.15:

$$
\delta(x)=4.64 \sqrt{\frac{x\left(v^{\prime}\right)}{v_{\infty}}}
$$

For our experimental conditions the average distance of the wafer from the leading edge is $0.5 \mathrm{~cm}$ and the average gas velocity is $35 \mathrm{~cm} / \mathrm{sec}$. These values give us an average boundary layer thickness of $0.68 \mathrm{~cm}$.

\section{REFERENCES-APPENDIX}

$1 \quad$ G.H. Geiger and D. R. Poirier, Transport Phenomena in Metallurgy, (Addison-Wesley Publishing Co., Menlo Park, CA 1980) p.11. 


\subsection{Recommendations For Future Research}

This program has identified ALE as a promising approach to high throughput, uniform growth of high efficiency solar cells. It is apparent from our work that device quality materials can be grown and that the uniformity of the materials is less dependent upon reactor design than conventional MOCVD. Furthermore, it appears that low growth temperatures are not only possible but necessary in order to achieve the self limiting aspects of ALE. In spite of these positive indications, several crucial questions were raised during the course of this work that will ultimately determine ALE's usefulness as a solar cell technology. These are listed below.

\subsection{Surface Reaction Rates}

The throughput of the ALE process is intimately tied to the surface reaction rates that control the reactions of metal alkyls and hydrides with the substrate surface. In the present study we were limited to ALE cycle times of four seconds. This translates to a growth rate of $\sim 0.25 \mu \mathrm{m} / \mathrm{hr}$. For a high throughput process, we suggest that growth rates as high as $2 \mu \mathrm{m} / \mathrm{hr}$ are necessary. To achieve these higher growth rates it will be necessary to measure the surface reaction rates for the organometallics and hydrides on GaAs surfaces to determine the optimal temperature range for the ALE process. We favor a direct measurement approach since we feel that our current limitations are partially affected by reactor design. A direct measurement of the surface reaction rates will determine the limits for the growth rate and will expose surface reaction processes. It may also lead to a reactor design philosophy that is not an obvious extension of current approaches. Furthermore, it will allow the direct measurement of the effect of optical excitation on the reaction rates to determine if this approach presents an opportunity for

\subsection{Reactor Design}

With the current reactor designs that operate at atmospheric or near atmospheric pressure, the high temperature limit and most likely the wafer geometry are limited by the onset of gas phase reactions that create nonsaturating surface species. To achieve the high throughput potential of ALE with organometallic precursors will require a significant rethinking of reactor design. Such a design may entail much lower pressure operation or it may require multiple reactant inlets to direct the reactants to the wafer surface. A significant effort must be placed in this area once the surface reactions and reaction rates are better understood.

\subsection{Materials Quality}

To date limited work has been directed to the improvement of materials quality grown by ALE or photoassisted ALE. Most of the effort has been directed to understanding the growth mechanisms and phenomenology. A directed effort of materials improvement and characterization must be undertaken since the quality of the materials will no doubt be dependent upon the growth parameters which, in turn, controls the throughput and economics of the process. This research should occur concurrently with mechanistic and reactor studies so that the implications of this research upon the quality of the materials is factored into research directions. 


\subsection{Students Supported and Publications of Program}

\section{Students Partially Supported By SERI During their Graduate Studies:}

1. H. C. Lee

2. Allen Danner

3. Ahsan Hariz

4. B.Y. Maa

5. Steve DenBaars

6. Eric Menū

(Post Doctoral Student)
Motorola

Applied Solar Energy Corporation - Advanced Optoelectronics

Not graduated

University of California at Santa Barbara

Motorola

\section{Papers Published Based on Work Partially Supported by SERI:}

1. S. P. DenBaars, C. A. Beyler, A. Hariz, and P. D. Dapkus, Appl. Phys Letters 51, 1530 (1987).

2. S. P. DenBaars, A. Hariz, C. Beyler, B.Y. Maa, and P. D. Dapkus, Mat. Res . Symp. Proc 102, 527 (1988).

3. P. D. Dapkus, S. P. DenBaars, Q. Chen, and B. Y. Maa, Proceedings of NATO Workshop on Reactions of Organometallics with Semiconductor Surfaces, in Mechanisms of Reactions of Organometallics Compound with Surfaces. D. J. ColeHamilton and J.O. Williams, eds., Proceedings of NATO Advanced Research Workshop, Vol. 198, Plenum Publishing Corp., New York (1989) pp 257-67 .

4. P. D. Dapkus, S. P. DenBaars, Q. Chen, W. G. Jeong, and B. Y. Maa, Prog. Crystal Growth and Charact. 19, 137 (1989)..

5. S. P. DenBaars, P. D. Dapkus, C. A. Beyler, A. Hariz, and K. M. Dzurko, J. Crystal Growth 93, 195 (1988).

6. S. P. DenBaars and P. D. Dapkus, J. Crystal Growth, J. Crystal Growth 98, 195 (1989).

7. W. G. Jeong, E. P. Menu and P. D. Dapkus, Proceedings of 1 st/nternational Conference on InP and Related Compounds, InP and Related Materials for Advanced Optoelectronic and Optical Devices, SPIE 1144, 86 (1989).

8. W. G. Jeong, E. P. Menu, and P. D. Dapkus, Mat. Res. Symp. Proc. 145, 163 (1989). 
9. S. P. DenBaars, P. D. Dapkus, C. A. Beyler, M. Zandian, A. Hariz, and K. M. Dzurko, Proceedings of the 1988 Symposium on GaAs and Related Compounds, Inst. Phys. Conf. Ser. No. 96, 89 (1989).

10. A. Hariz, P. D. Dapkus, H. C. Lee, E. P. Menu, adn S. P. DenBaars, Appl. Phys Lett. 54,635 (1989).

11. W. G. Jeong, E. P. Menu and P. D. Dapkus, Appl. Phys. Lett. 55, 244 (1989).

12. S. P. DenBaars, P. D. Dapkus and A. Melas, J. Electrochem. Soc. 136, 2067 (1989)

END DATE

$$
5-25-93
$$




\begin{tabular}{|c|c|c|c|}
\hline $\begin{array}{c}\text { Document Control } \\
\text { Page }\end{array}$ & $\begin{array}{l}\text { 1. NREL Report No. } \\
\text { NREL/TP-451-4853 }\end{array}$ & $\begin{array}{l}\text { 2. NTIS Accession No. } \\
\text { DE92010562 }\end{array}$ & 3. Recipient's Accession No. \\
\hline \multirow{2}{*}{\multicolumn{3}{|c|}{$\begin{array}{l}\text { 4. Title and Subtitle } \\
\text { Low-Temperature Metal-Organic Chemical Vapor Deposition Growth Processes } \\
\text { for High-Efficiency Solar Cells }\end{array}$}} & $\begin{array}{c}\text { 5. Publication Date } \\
\text { February } 1993 \\
\end{array}$ \\
\hline & & & 6. \\
\hline \multicolumn{3}{|l|}{$\begin{array}{l}\text { 7. Author(s) } \\
\text { P. D. Dapkus }\end{array}$} & 8. Performing Organization Rept. No. \\
\hline \multirow{2}{*}{\multicolumn{3}{|c|}{$\begin{array}{l}\text { 9. Performing Organization Name and Address } \\
\text { University of Southern California } \\
\text { Departments of Electrical Engineering and Materials Science } \\
\text { Los Angeles, California 90089-0483 }\end{array}$}} & $\begin{array}{l}\text { 10. Project/TaskMork Unit No. } \\
\text { PV221103 }\end{array}$ \\
\hline & & & $\begin{array}{l}\text { 11. Contract (C) or Grant (G) No. } \\
\text { (C) XB-5-05009-3 } \\
\text { (G) }\end{array}$ \\
\hline \multirow{2}{*}{\multicolumn{3}{|c|}{$\begin{array}{l}\text { 12. Sponsoring Organization Name and Address } \\
\text { National Renewable Energy Laboratory } \\
1617 \text { Cole Blvd. } \\
\text { Golden, CO } 80401-3393\end{array}$}} & $\begin{array}{l}\text { 13. Type of Report \& Period Covered } \\
\text { Technical Report } \\
\text { 1 September } 1985-30 \text { November } \\
1989\end{array}$ \\
\hline & & & 14. \\
\hline \multicolumn{4}{|c|}{$\begin{array}{l}\text { 15. Supplementary Notes } \\
\text { NREL technical monitor: J. Benner }\end{array}$} \\
\hline \multicolumn{4}{|c|}{$\begin{array}{l}\text { 16. Abstract (Limit: } 200 \text { words) } \\
\text { This report describes the results of a program to develop a more complete understanding of the physical and chemical } \\
\text { processes involved in low-temperature growth of III-V compounds by metal-organic chemical vapor deposition (MOCVD) and } \\
\text { to develop a low-temperature process that is suitable for the growth of high-efficiency solar cells. The program was structured } \\
\text { to develop a better understanding of the chemical reactions involved in MOCVD growth, to develop a model of the processes } \\
\text { occurring in the gas phase, to understand the physical kinetics and reactions operative on the surface of the growing crystal, } \\
\text { and to develop an understanding of the means by which these processes may be altered to reduce the temperature of growth } \\
\text { and the utilization of toxic hydrides. The basic approach was to develop the required information about the chemical and } \\
\text { physical kinetics operative in the gas phase and on the surface by the direct physical measurement of the processes whenever } \\
\text { possible. The program included five tasks: (1) MOCVD growth process characterization, (2) photoenhanced MOCVD } \\
\text { studies, (3) materials characterization, (4) device fabrication and characterization, and (5) photovoltaic training. Most of the } \\
\text { goals of the program were met, and significant progress was made in defining an approach that would allow both high } \\
\text { throughput and high uniformity growth of compound semiconductors at low temperatures. The technical activity was focused } \\
\text { on determining the rates of thermal decomposition of trimethyl gallium, exploring alternate arsenic sources for use in } \\
\text { MOCVD, and empirical studies of atomic layer epitaxy as an approach. }\end{array}$} \\
\hline \multicolumn{4}{|c|}{$\begin{array}{l}\text { 17. Document Analysis } \\
\text { a. Descriptors } \\
\text { low temperature ; MOCVD ; high efficiency ; photovoltaics ; solar cells ; metal organic ; chemical vapor deposition } \\
\text { b. Identifiers/Open-Ended Terms } \\
\text { c. UC Categories } \\
273\end{array}$} \\
\hline \multirow{2}{*}{\multicolumn{3}{|c|}{$\begin{array}{l}\text { 18. Availability Statement } \\
\text { National Technical Information Service } \\
\text { U.S. Department of Commerce } \\
\text { 5285 Port Royal Road } \\
\text { Springfield, VA } 22161\end{array}$}} & $\begin{array}{l}\text { 19. No. of Pages } \\
297\end{array}$ \\
\hline & & & $\begin{array}{r}\text { 20. Price } \\
\text { A13 }\end{array}$ \\
\hline
\end{tabular}

Form No. 0069E (6-30-87) 